\title{
HECKE ALGEBRAS WITH UNEQUAL PARAMETERS
}

\author{
G. Lusztig
}

\section{INTRODUCTION}

These notes are an expanded version of the Aisenstadt lectures given at the CRM, Université de Montréal, in May/June 2002; they also include material from lectures given at MIT during the Fall of 1999 [L12]. I wish to thank Jacques Hurtubise for inviting me to give the Aisenstadt lectures.

Hecke algebras arise as endomorphism algebras of representations of groups induced by representations of subgroups. In these notes we are mainly interested in a particular kind of Hecke algebras, which arise in the representation theory of reductive algebraic groups over finite or $p$-adic fields (see $0.3,0.6$ ). These Hecke algebras are specializations of certain algebras (known as Iwahori-Hecke algebras) which can be defined without reference to algebraic groups, namely by explicit generators and relations (see 3.2) in terms of a Coxeter group $W$ (see 3.1) and a weight function $L: W \rightarrow \mathbf{Z}$ (see 3.1), that is, a weighted Coxeter group. An Iwahori-Hecke algebra is completely specified by a weighted Coxeter graph, that is, the Coxeter graph of $W$ (see 1.1) where for each vertex we specify the value of $L$ at the corresponding simple reflection.

A particularly simple kind of Iwahori-Hecke algebras corresponds to the case where the weight function is constant on the set of simple reflections (equal parameter case). In this case one has the theory of the "new basis" [KL1] and cells [KL1], [L6], [L8]. The main goal of these notes is to try to extend as much as possible the theory of the new basis to the general case (of not necessarily equal parameters). We give a number of conjectures for what should happen in the general case and we present some evidence for these conjectures.

We now review the contents of these notes.

$\S 1$ introduces Coxeter groups following [Bo]. We also give a realization of the classical affine Weyl groups as periodic permutations of $\mathbf{Z}$ following an idea of [L4]. $\S 2$ contains some standard results on the partial order on a Coxeter group. In $\S 3$ we introduce the Iwahori-Hecke algebra attached to a weighted Coxeter group. Useful references for this are [Bo],[GP]. In $\S 4$ we define the bar operator following [KL1]. This is used in $\S 5$ to define the "new basis" $\left(c_{w}\right)$ of an Iwahori-Hecke

Supported in part by the National Science Foundation

Typeset by $\mathcal{A M}_{\mathcal{M}}-\mathrm{T}_{\mathrm{E}} \mathrm{X}$ 
algebra following [KL1] for equal parameters and [L3] in general. In $§ 6$ we study some multiplicative properties of the new basis, following [KL1] and [L3]. In $\S 7$ we compute explicitly the "new basis" in the case of dihedral groups. In $\S 8$ we define left, right and two-sided cells. In $\S 9$ we study the behaviour of the new basis in relation to a given parabolic subgroup. In $§ 10, \S 12$ we study a "basis" dual to the new basis. In $\S 11$ we consider the case of finite Coxeter groups. In $\S 13$ we study the function a on certain weighted Coxeter groups following an idea from [L6]. In $\S 14$ we present a list of conjectures concerning cells and the function a and we show that they can be deduced from a much shorter list of conjectures. These conjectures are established in a "split case" in $\S 15$ (following [L8]), in a "quasisplit case" in $\S 16$ and for an infinite dihedral group in $\S 17$. Note that in the first two cases the proof requires arguments from intersection cohomology while in the third case the argument is computational. In $\S 18$, assuming the truth of these conjectures we develop the theory of "asymptotic Hecke algebras" $J$ in the weighted case, following an idea from [L8]. $§ 19, \S 20, \S 21$ (where $W$ is assumed to be a Weyl group) are in preparation for $\S 22$ where the class of constructible representations of $W$ is introduced and studied in the weighted case (conjecturally these are the representations of $W$ carried by left cells), for $\S 23$ where two-sided cells are discussed and for $\S 24$ where certain virtual representations of $W$ ("virtual cells) are discussed. In $\S 25$ we discuss the weighted Coxeter groups which arise in the examples 0.3 and 0.6. We formulate a conjecture (25.3) which relates the two-sided cells of such a weighted Coxeter group to the two-sided cells of a larger Coxeter group with the weight function given by the length. In $\S 26$ we state (following [L13]) the classification of irreducible representations of Hecke algebras of the type discussed in 0.6 in terms of the geometry of the dual group. In $\S 27$ we give a new realization of a Hecke algebra as in 0.3 or 0.6 as a space of functions on the rational points of an algebraic variety defined over $\mathbf{F}_{q}$. This leads us to a (partly conjectural) geometrical interpretation of the coefficients $p_{y, w}$ of the new basis of the Hecke algebra in terms of intersection cohomology, generalizing the results of [KL2]. We expect that this geometrical interpretation should play a role in the proof of the conjectures in $\S 14$ in the cases arising from algebraic groups as in $0.3,0.6$.

0.1. In $0.1-0.8$ we give a survey of the theory of Hecke algebras arising from reductive groups.

Let $\Gamma$ be a group acting transitively on a set $X$. If $\mathbf{E}$ is a $\Gamma$-equivariant $\mathbf{C}$ vector bundle over $X$ (with discrete topology) then the fibre $\mathbf{E}_{x}$ of $\mathbf{E}$ at $x \in X$ is naturally a representation of $\Gamma_{x}=\{g \in \Gamma ; g x=x\}$. Moreover, for $x \in X, \mathbf{E} \mapsto \mathbf{E}_{x}$ is an equivalence from the category of $\Gamma$-equivariant vector bundles on $X$ of finite dimension and that of finite dimensional $\Gamma_{x}$-modules over $\mathbf{C}$.

Let $\mathbf{E}$ be a $\Gamma$-equivariant $\mathbf{C}$-vector bundle of finite dimension over $X$. Then $\Gamma$ acts naturally on the vector space $\oplus_{x \in X} \mathbf{E}_{x}$. (This is the representation of $\Gamma$ induced by the representation of $\Gamma_{x}$ on $\mathbf{E}_{x}$, for any $x \in X$.) The $\mathbf{C}$-algebra

$$
H=H(\Gamma, X, \mathbf{E})=\operatorname{End}_{\Gamma}\left(\oplus_{x \in X} \mathbf{E}_{x}\right)
$$


is called the Hecke algebra. The image of the obvious imbedding

$$
H \subset \prod_{\left(x, x^{\prime}\right) \in X \times X} \operatorname{Hom}\left(\mathbf{E}_{x}, \mathbf{E}_{x^{\prime}}\right), \quad \phi \mapsto\left(\phi_{x^{\prime}}^{x}\right)_{\left(x, x^{\prime}\right) \in X \times X}
$$

consists of all $\left(f_{x^{\prime}}^{x}\right) \in \prod_{\left(x, x^{\prime}\right) \in X \times X} \operatorname{Hom}\left(\mathbf{E}_{x}, \mathbf{E}_{x^{\prime}}\right)$ such that

for any $x \in X$ we have $f_{x^{\prime}}^{x}=0$ for all but finitely many $x^{\prime} \in X$;

for any $g \in \Gamma$ and any $\left(x, x^{\prime}\right) \in X \times X$, the compositions $\mathbf{E}_{x} \stackrel{f_{x^{\prime}}^{x}}{\longrightarrow} \mathbf{E}_{x^{\prime}} \stackrel{g}{\rightarrow} \mathbf{E}_{g x^{\prime}}$, $\mathbf{E}_{x} \stackrel{g}{\rightarrow} \mathbf{E}_{g x} \stackrel{f_{g x^{\prime}}^{g x}}{\longrightarrow} \mathbf{E}_{g x^{\prime}}$ coincide.

For any $\Gamma$-orbit $\mathcal{C}$ in $X \times X$ we set

$$
H_{\mathcal{C}}=H(\Gamma, X, \mathbf{E})_{\mathcal{C}}=\left\{\phi \in H ; \phi_{x^{\prime}}^{x} \neq 0 \Longrightarrow\left(x^{\prime}, x\right) \in \mathcal{C}\right\} .
$$

Then $H_{\mathcal{C}}=0$ unless $\mathcal{C}$ is finitary in the following sense:

for some (or any) $x \in X$, the set $\left\{x^{\prime} \in X ;\left(x^{\prime}, x\right) \in \mathcal{C}\right\}$ is finite, in which case

$$
H_{\mathcal{C}} \stackrel{\sim}{\rightarrow} \operatorname{Hom}_{\Gamma_{x} \cap \Gamma_{x^{\prime}}}\left(\mathbf{E}_{x}, \mathbf{E}_{x^{\prime}}\right), \quad \phi \mapsto \phi_{x^{\prime}}^{x}
$$

for $\left(x^{\prime}, x\right) \in \mathcal{C}$. Moreover,

(a) $H=\oplus_{\mathcal{C}}$ finitary $H_{\mathcal{C}}$.

0.2. To explain how Hecke algebras arise from reductive algebraic groups we need the notion of "unipotent cuspidal representation".

Let $p$ be a prime number and let $\mathbf{F}$ be an algebraic closure of the finite field with $p$ elements. Let $q$ be a power of $p$ and let $\mathbf{F}_{q}$ be the subfield of $\mathbf{F}$ with $q$ elements. Let $G$ be a connected reductive algebraic group over $\mathbf{F}$ with a fixed $\mathbf{F}_{q}$ structure and let $F: G \rightarrow G$ be the corresponding Frobenius map.

We refer to [DL] for the notion of unipotent cuspidal representation of the finite group $G^{F}=G\left(\mathbf{F}_{q}\right)$. We will only give here the definition assuming that $q$ is sufficiently large. Let $E$ be an irreducible representation over $\mathbf{C}$ of $G^{F}$ and let $\chi_{E}: G^{F} \rightarrow \mathbf{C}$ be its character. We say that $E$ is unipotent if, for any $F$-stable maximal torus $T$ of $G$, the restriction of $\chi_{E}$ to the set of regular elements in $T^{F}$ is a constant, say $c_{T} \in \mathbf{Z}$. We say that $E$ is unipotent cuspidal if, in addition, for any $T$ as above that is contained in some proper $F$-stable parabolic subgroup of $G$, we have $c_{T}=0$.

The unipotent cuspidal representations of $G^{F}$ are classified in [L5]. For example, if $G$ is a torus times a symplectic group of rank $n \geq 0$ then $G^{F}$ has (up to isomorphism) a unique unipotent cuspidal representation if $n=k^{2}+k$ for some integer $k \geq 0$, and none, otherwise.

0.3. Let $G, F$ be as in 0.2 . For any parabolic subgroup $P$ of $G$ let $U_{P}$ be the unipotent radical of $P$ and let $\bar{P}=P / U_{P}$. Let $\mathcal{P}$ be an $F$-stable $G$-conjugacy class of parabolic subgroups of $G$ and let $\mathbf{E}$ be a $G^{F}$-equivariant vector bundle over $\mathcal{P}^{F}$ (a $G^{F}$-homogeneous space) such that for some (or any) $P \in \mathcal{P}^{F}$, the 
$P^{F}$-action on the fibre $\mathbf{E}_{P}$ of $\mathbf{E}$ at $P$ factors through a unipotent, cuspidal $\bar{P}^{F}$ module. (To give such $\mathbf{E}$ is the same as to give, for some $P \in \mathcal{P}^{F}$, a unipotent cuspidal representation of $\bar{P}^{F}$.) The Hecke algebra $H\left(G^{F}, \mathcal{P}^{F}, \mathbf{E}\right)$ is defined.

Let $W$ be the set of $G$-orbits on the set of ordered pairs of Borel subgroups in $G$; it is known that $W$ may be naturally regarded as a finite Coxeter group (see 1.1) with a set $S$ of simple reflections. Now any Borel subgroup of $G$ is contained in a unique subgroup in $\mathcal{P}$; this defines a (surjective) map from $W$ to $G \backslash(\mathcal{P} \times \mathcal{P})$, the set of $G$-orbits in $\mathcal{P} \times \mathcal{P}$. The inverse image of the diagonal orbit under this map is the subgroup $W_{J}$ of $W$ generated by a subset $J$ of $S$ and $W \rightarrow G \backslash(\mathcal{P} \times \mathcal{P})$ factors through a bijection

$$
W_{J} \backslash W / W_{J} \stackrel{\sim}{\longrightarrow} G \backslash(\mathcal{P} \times \mathcal{P}) .
$$

Let $\mathcal{W}$ be the set of all $w \in W$ such that $w W_{J}=W_{J} w$ and $w$ has minimal length in $w W_{J}=W_{J} w$. Then $\mathcal{W}$ is a subgroup of $W$. The Frobenius map $u: W \rightarrow W$ restricts to an isomorphism $u: \mathcal{W} \rightarrow \mathcal{W}$ whose fixed point set $\mathcal{W}^{u}$ is naturally a Coxeter group with simple reflections indexed by $u \backslash(S-J)$ (set of orbits of $u: S-J \rightarrow S-J)$. See 25.1(a). A $G$-orbit $\mathcal{O}$ on $\mathcal{P} \times \mathcal{P}$ is said to be good if for $\left(P, P^{\prime}\right) \in \mathcal{O}$ we have $\left(P \cap P^{\prime}\right) U_{P}=P$ or equivalently $\left(P \cap P^{\prime}\right) U_{P^{\prime}}=P^{\prime}$. Otherwise, $\mathcal{O}$ is said to be bad. If $\mathcal{O}$ is a good, $F$-stable $G$-orbit on $\mathcal{P} \times \mathcal{P}$ then $\mathcal{O}^{F}$ is a $G^{F_{-}}$ orbit on $\mathcal{P}^{F} \times \mathcal{P}^{F}$ and $\operatorname{dim} H\left(G^{F}, \mathcal{P}^{F}, \mathbf{E}\right)_{\mathcal{O}^{F}}=1$. If $\mathcal{O}$ is an $F$-stable bad $G$-orbit on $\mathcal{P} \times \mathcal{P}$ then $\mathcal{O}^{F}$ is a $G^{F}$-orbit on $\mathcal{P}^{F} \times \mathcal{P}^{F}$ and $\operatorname{dim} H\left(G^{F}, \mathcal{P}^{F}, \mathbf{E}\right)_{\mathcal{O}^{F}}=0$. Now the bijection (a) restricts (via the imbedding $\mathcal{W}^{u} \subset W_{J} \backslash W / W_{J}, w \mapsto W_{J} w W_{J}$ ) to a bijection $w \mapsto \mathcal{O}_{w}$ of $\mathcal{W}^{u}$ onto the set of good, $F$-stable $G$-orbits on $\mathcal{P} \times \mathcal{P}$. It follows that $0.1(\mathrm{a})$ becomes in our case

$$
H\left(G^{F}, \mathcal{P}^{F}, \mathbf{E}\right)=\oplus_{w \in \mathcal{W}^{u}} H\left(G^{F}, \mathcal{P}^{F}, \mathbf{E}\right)_{\mathcal{O}_{w}}
$$

with

$$
\operatorname{dim} H\left(G^{F}, \mathcal{P}^{F}, \mathbf{E}\right)_{\mathcal{O}_{w}}=1 \text { for all } w \in \mathcal{W}^{u} .
$$

Let $\tau_{k}$ be the generator of $\mathcal{W}^{u}$ corresponding to $k \in u \backslash(S-J)$. There is a unique basis element $T_{\tau_{k}}$ of $H\left(G^{F}, \mathcal{P}^{F}, \mathbf{E}\right)_{\mathcal{O}_{\tau_{k}}}$ such that

$$
\left(T_{\tau_{k}}+q^{-N_{k} / 2}\right)\left(T_{\tau_{k}}-q^{N_{k} / 2}\right)=0
$$

for some $N_{k} \in \mathbf{Z}_{>0}$. ( $N_{k}$ is uniquely determined.) The elements $T_{\tau_{k}}(k \in u \backslash(S-J))$ generate the $\mathbf{C}$-algebra $H\left(G^{F}, \mathcal{P}^{F}, \mathbf{E}\right)$. They satisfy identities of the form

$$
T_{\tau_{k}} T_{\tau_{k^{\prime}}} T_{\tau_{k}} \cdots=T_{\tau_{k^{\prime}}} T_{\tau_{k}} T_{\tau_{k^{\prime}}} \ldots
$$

for $k \neq k^{\prime}$ in $u \backslash(S-J)$; both products have a number of factors equal to the order of $\tau_{k} \tau_{k^{\prime}}$ in $\mathcal{W}^{u}$. Now $T_{\tau_{k}} \mapsto T_{\tau_{k}}$ gives an isomorphism from an Iwahori-Hecke algebra (see 3.2) specialized at $v=\sqrt{q}$ to the algebra $H\left(G^{F}, \mathcal{P}^{F}, \mathbf{E}\right)$.

The function $k \mapsto N_{k}$ coincides with the function $k \mapsto L\left(\tau_{k}\right)$ in 25.2.

(The results in this subsection appeared in [L2,L1]. In the special case where $\mathcal{P}$ is the set of Borel subgroups of $G$ and $\mathbf{E}$ is the trivial vector bundle $\mathbf{C}$, they were first proved by Iwahori [I]; if, in addition, $u=1$ on $W$ then $N_{k}=1$ for all $k$.) 
0.4. Let $V$ be an $\mathbf{F}_{q}$-vector space of dimension $n \geq 2$. Then $G=S L(\mathbf{F} \otimes$

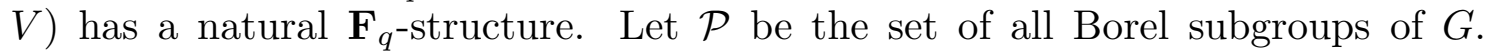
Then $\mathcal{P}^{F}$ may be identified as a set with $G^{F}$-action with the set $\mathcal{F}$ of all flags $V_{*}=\left(V_{0} \subset V_{1} \subset V_{2} \subset \ldots \subset V_{n}\right)$ of subspaces of $V\left(\operatorname{dim} V_{i}=i\right.$ for all $\left.i\right)$.

Let $V_{*}=\left(V_{0} \subset V_{1} \subset V_{2} \subset \ldots \subset V_{n}\right), V_{*}^{\prime}=\left(V_{0}^{\prime} \subset V_{1}^{\prime} \subset V_{2}^{\prime} \subset \ldots \subset V_{n}^{\prime}\right)$ be flags in $\mathcal{F}$. For $i \in[0, n], j \in[1, n]$ we set $d_{i j}=\operatorname{dim} \frac{V_{i}^{\prime} \cap V_{j}}{V_{i}^{\prime} \cap V_{j-1}} \in\{0,1\}$. For $i \in[0, n]$ we set $X_{i}=\left\{j \in[1, n] ; d_{i j}=1\right\}$. Then $\emptyset=X_{0} \subset X_{1} \subset X_{2} \subset \ldots \subset X_{n}=[1, n]$ and for $i \in[1, n]$ there is a unique $a_{i} \in[1, n]$ such that $X_{i}=X_{i-1} \sqcup\left\{a_{i}\right\}$. Also, $i \mapsto a_{i}$ is a permutation of $[1, n]$. Now $\left(V_{*}, V_{*}^{\prime}\right) \mapsto\left(a_{i}\right)$ defines a bijection of $G^{F} \backslash\left(\mathcal{P}^{F} \times \mathcal{P}^{F}\right)=G^{F} \backslash(\mathcal{F} \times \mathcal{F})$ with the symmetric group $\mathfrak{S}_{n}$. Let $\mathbf{E}$ be the trivial $G^{F}$-equivariant vector bundle $\mathbf{C}$ on $\mathcal{P}^{F}=\mathcal{F}$. Then $H\left(G^{F}, \mathcal{P}^{F}, \mathbf{E}\right)$ is defined. In our case we have $W=\mathcal{W}=\mathcal{W}^{u}=\mathfrak{S}_{n}$.

0.5. Let $V, n$ be as in 0.4 . Assume that $n=2 m$ and that $V$ has a fixed nondegenerate symplectic form $\langle\rangle:, V \times V \rightarrow \mathbf{F}_{q}$. Then $G=S p(\mathbf{F} \otimes V)$ has a natural $\mathbf{F}_{q}$-structure. Assume that $m=r+k^{2}+k$ where $k \in \mathbf{N}, r \in \mathbf{Z}_{>0}$. Let $\mathcal{F}$ be the set of all flags $V_{*}=\left(V_{0} \subset V_{1} \subset V_{2} \subset \ldots \subset V_{r}\right)$ of isotropic subspaces of $V\left(\operatorname{dim} V_{i}=i\right.$ for all $i$ ). There is a unique $G$-conjugacy class $\mathcal{P}$ of parabolic subgroups of $G$ such that, if $V_{*} \in \mathcal{F}$, then

$$
\left\{g \in G ; g\left(\mathbf{F} \otimes V_{j}\right)=\mathbf{F} \otimes V_{j} \quad \forall j\right\} \in \mathcal{P} .
$$

We may identify $\mathcal{P}^{F}=\mathcal{F}$ as spaces with $G^{F}$-action.

Let $U \mapsto \mathcal{D}_{k}(U)$ be a functor from the category of symplectic vector spaces of dimension $2 k^{2}+2 k$ over $\mathbf{F}_{q}$ (and isomorphisms between them) to the category of $\mathbf{C}$-vector spaces (and isomorphisms between them) such that for any $U$, the $S p(U)$-module $\mathcal{D}_{k}(U)$ is unipotent, cuspidal. (Such a functor exists and is unique up to isomorphism.) Let $\mathbf{E}$ be the vector bundle over $\mathcal{P}^{F}$ (or equivalently $\mathcal{F}$ ) whose fibre at $V_{*}=\left(V_{0} \subset V_{1} \subset V_{2} \subset \ldots \subset V_{r}\right) \in \mathcal{F}^{F}$ is $\mathcal{D}_{k}\left(V_{r}^{\perp} / V_{r}\right)$. (Here $V_{s}^{\perp}=\left\{x \in V ;\left\langle x, V_{s}\right\rangle=0\right\}$.)

This vector bundle is naturally $G^{F}$-equivariant (since $\mathcal{D}_{k}$ is a functor). Hence $H\left(G^{F}, \mathcal{P}^{F}, \mathbf{E}\right)$ is defined.

Let $V_{*}=\left(V_{0} \subset V_{1} \subset V_{2} \subset \ldots \subset V_{r}\right), V_{*}^{\prime}=\left(V_{0}^{\prime} \subset V_{1}^{\prime} \subset V_{2}^{\prime} \subset \ldots \subset V_{r}^{\prime}\right)$ be flags in $\mathcal{F}$. The $G$-orbit of the point of $\mathcal{P} \times \mathcal{P}$ corresponding to $\left(V_{*}, V_{*}^{\prime}\right)$ is good if the following three equivalent conditions hold:

$$
\begin{aligned}
& V_{r} \cap V_{r}^{\prime}=V_{r} \cap V_{r}^{\prime \perp}, \\
& V_{r} \cap V_{r}^{\prime}=V_{r}^{\perp} \cap V_{r}^{\prime}, \\
& V_{r} \cap V_{r}^{\prime}=\left(V_{r}^{\perp} \cap V_{r}^{\prime \perp}\right) \cap\left(V_{r}+V_{r}^{\prime}\right) .
\end{aligned}
$$

If these conditions hold, we can define an isomorphism $\psi_{V_{r}^{\prime}}^{V_{r}}: V_{r}^{\perp} / V_{r} \rightarrow V_{r}^{\prime \perp} / V_{r}^{\prime}$ by requiring that the diagram

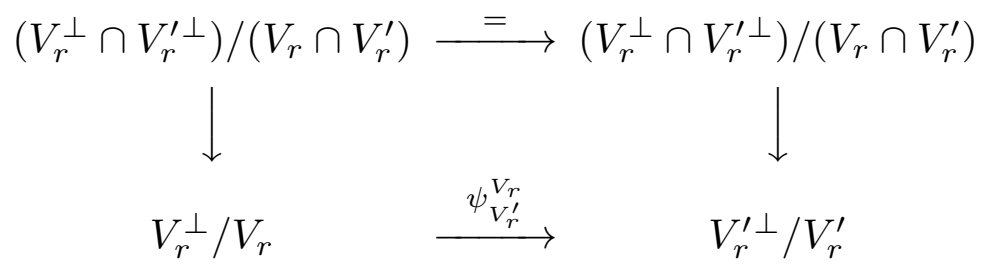


(where the vertical maps are the isomorphisms induced by the inclusion) is commutative. In this case, to $\left(V_{*}, V_{*}^{\prime}\right)$ we associate an element $\sigma$ of the group $W_{r}$ of permutations of $\mathcal{S}=\left\{1,2, \ldots, r, r^{\prime}, \ldots, 2^{\prime}, 1^{\prime}\right\}$ that commute with the involution $f: \mathcal{S} \rightarrow \mathcal{S}, j \mapsto j^{\prime}, j^{\prime} \mapsto j$. For $j \in[1, r]$ we set

$A_{j}=\left\{h \in[1, r] ; V_{h-1} \cap V_{j}^{\prime} \neq V_{h} \cap V_{j}^{\prime}\right\}, \quad B_{j}=f\left\{h \in[1, r] ; V_{h-1}^{\perp} \cap V_{j}^{\prime} \neq V_{h}^{\perp} \cap V_{j}^{\prime}\right\}$.

Then $\sharp\left(A_{j} \cap B_{j}\right)=j, A_{1} \cap B_{1} \subset A_{2} \cap B_{2} \subset \ldots \subset A_{s} \cap B_{s}$ and $h \in A_{j} \Longrightarrow h^{\prime} \notin B_{j}$. For $j \in[1, r]$ define $a_{j} \in \mathcal{S}$ by $A_{j} \cup B_{j}=\left\{a_{1}, a_{2}, \ldots, a_{j}\right\}$. Then $\sigma$ is defined by the condition that $\sigma(j)=a_{j}$ for $j \in[1, r]$. We see that in our case, $\mathcal{W}=\mathcal{W}^{u}$ may be identified with $W_{r}$. In our case, the Iwahori-Hecke algebra corresponds to the weighted Coxeter graph

$$
\bullet_{2 k+1}=\bullet_{1}-\bullet_{1}-\cdots-\bullet_{1}
$$

( $r$ vertices); in the case where $r=1$ this should be interpreted as a graph with one vertex marked by $2 k+1$.

0.6. Let $\epsilon$ be an indeterminate. Let $\mathbf{K}$ be the subfield of $\mathbf{F}((\epsilon))$ generated by $\mathbf{F}_{q}((\epsilon))$ and $\mathbf{F}$. Let $\mathbf{G}$ be a split connected simply connected almost simple algebraic group over $\mathbf{K}$ with a fixed $\mathbf{F}_{q}((\epsilon))$-rational structure. We identify $\mathbf{G}$ with its group of $\mathbf{K}$-points. There is a "Frobenius map" $F: \mathbf{G} \rightarrow \mathbf{G}$ whose fixed point set is $\mathbf{G}\left(\mathbf{F}_{q}((\epsilon))\right)$. Let $\mathcal{B}$ be the set of all Iwahori subgroups of $\mathbf{G}$. (This concept will be illustrated in 0.7.) A subgroup of $\mathbf{G}$ is said to be a parahoric subgroup if it is $\neq \mathbf{G}$ and it contains some Iwahori subgroup. If $P$ is a parahoric subgroup then $P$ has a "pro-unipotent radical" $U_{P}$ and $\bar{P}=P / U_{P}$ is a connected, reductive group over $\mathbf{F}$. Let $\mathcal{P}$ be an $F$-stable $\mathbf{G}$-conjugacy class of parahoric subgroups of $\mathbf{G}$ and let $\mathbf{E}$ be a $\mathbf{G}^{F}$-equivariant vector bundle over $\mathcal{P}^{F}$ (a $\mathbf{G}^{F}$-homogeneous space) such that for some (or any) $P \in \mathcal{P}^{F}$, the $P^{F}$-action on the fibre $\mathbf{E}_{P}$ of $\mathbf{E}$ at $P$ factors through a unipotent, cuspidal $\bar{P}^{F}$-module. (To give such $\mathbf{E}$ is the same as to give, for some $P \in \mathcal{P}^{F}$, a unipotent cuspidal representation of $\bar{P}^{F}$.) The Hecke algebra $H\left(\mathbf{G}^{F}, \mathcal{P}^{F}, \mathbf{E}\right)$ is defined.

Let $W$ be the set of $\mathbf{G}$-orbits on $\mathcal{B} \times \mathcal{B}$; it is known that $W$ may be naturally regarded as a Coxeter group (more precisely, an affine Weyl group, see 1.15) with a set $S$ of simple reflections. Now any Iwahori subgroup of $\mathbf{G}$ is contained in a unique subgroup in $\mathcal{P}$; this defines a (surjective) map from $W$ to $\mathbf{G} \backslash(\mathcal{P} \times \mathcal{P})$, the set of $\mathbf{G}$-orbits in $\mathcal{P} \times \mathcal{P}$. The inverse image of the diagonal orbit under this map is the subgroup $W_{J}$ of $W$ generated by a subset $J$ of $S$ and $W \rightarrow \mathbf{G} \backslash(\mathcal{P} \times \mathcal{P})$ factors through a bijection

$$
W_{J} \backslash W / W_{J} \stackrel{\sim}{\longrightarrow} \mathbf{G} \backslash(\mathcal{P} \times \mathcal{P})
$$

Let $\mathcal{W}$ be the set of all $w \in W$ such that $w W_{J}=W_{J} w$ and $w$ has minimal length in $w W_{J}=W_{J} w$. Then $\mathcal{W}$ is a subgroup of $W$. The Frobenius map $u: W \rightarrow W$ 
restricts to an isomorphism $u: \mathcal{W} \rightarrow \mathcal{W}$ whose fixed point set $\mathcal{W}^{u}$ is naturally an infinite Coxeter group with simple reflections indexed by $u \backslash(S-J)$ (set of orbits of $u: S-J \rightarrow S-J)$. (We make the additional assumption that $\sharp(u \backslash(S-J)) \geq 2$.) See 25.1(a). A G-orbit $\mathcal{O}$ on $\mathcal{P} \times \mathcal{P}$ is said to be good if for $\left(P, P^{\prime}\right) \in \mathcal{O}$ we have $\left(P \cap P^{\prime}\right) U_{P}=P$ or equivalently $\left(P \cap P^{\prime}\right) U_{P^{\prime}}=P^{\prime}$. Otherwise, $\mathcal{O}$ is said to be $b a d$. If $\mathcal{O}$ is a good, $F$-stable $\mathbf{G}$-orbit on $\mathcal{P} \times \mathcal{P}$ then $\mathcal{O}^{F}$ is a $\mathbf{G}^{F}$-orbit on $\mathcal{P}^{F} \times \mathcal{P}^{F}$ and $\operatorname{dim} H\left(\mathbf{G}^{F}, \mathcal{P}^{F}, \mathbf{E}\right)_{\mathcal{O}^{F}}=1$. If $\mathcal{O}$ is an $F$-stable bad $\mathbf{G}$-orbit on $\mathcal{P} \times \mathcal{P}$ then $\mathcal{O}^{F}$ is a $\mathbf{G}^{F}$-orbit on $\mathcal{P}^{F} \times \mathcal{P}^{F}$ and $\operatorname{dim} H\left(\mathbf{G}^{F}, \mathcal{P}^{F}, \mathbf{E}\right)_{\mathcal{O}^{F}}=0$. Now the bijection (a) restricts (via the imbedding $\mathcal{W}^{u} \subset W_{J} \backslash W / W_{J}, w \mapsto W_{J} w W_{J}$ ) to a bijection $w \mapsto \mathcal{O}_{w}$ of $\mathcal{W}^{u}$ onto the set of good, $F$-stable $\mathbf{G}$-orbits on $\mathcal{P} \times \mathcal{P}$. It follows that 0.1 (a) becomes in our case

$$
H\left(\mathbf{G}^{F}, \mathcal{P}^{F}, \mathbf{E}\right)=\oplus_{w \in \mathcal{W}^{u}} H\left(\mathbf{G}^{F}, \mathcal{P}^{F}, \mathbf{E}\right)_{\mathcal{O}_{w}}
$$

with

$$
\operatorname{dim} H\left(\mathbf{G}^{F}, \mathcal{P}^{F}, \mathbf{E}\right)_{\mathcal{O}_{w}}=1 \text { for all } w \in \mathcal{W}^{u}
$$

Let $\tau_{k}$ be the generator of $\mathcal{W}^{u}$ corresponding to $k \in u \backslash(S-J)$. There is a unique basis element $T_{\tau_{k}}$ of $H\left(\mathbf{G}^{F}, \mathcal{P}^{F}, \mathbf{E}\right)_{\mathcal{O}_{\tau_{k}}}$ such that

$$
\left(T_{\tau_{k}}+q^{-N_{k} / 2}\right)\left(T_{\tau_{k}}-q^{N_{k} / 2}\right)=0
$$

for some $N_{k} \in \mathbf{Z}_{>0}$. ( $N_{k}$ is uniquely determined.) The elements $T_{\tau_{k}}(k \in u \backslash(S-J))$ generate the $\mathbf{C}$-algebra $H\left(\mathbf{G}^{F}, \mathcal{P}^{F}, \mathbf{E}\right)$. They satisfy identities of the form

$$
T_{\tau_{k}} T_{\tau_{k^{\prime}}} T_{\tau_{k}} \cdots=T_{\tau_{k^{\prime}}} T_{\tau_{k}} T_{\tau_{k^{\prime}}} \ldots
$$

for $k \neq k^{\prime}$ in $u \backslash(S-J)$ with $\tau_{k} \tau_{k^{\prime}}$ of order $m_{k, k^{\prime}}<\infty$ in $\mathcal{W}^{u}$ (both products have $m_{k, k^{\prime}}$ factors). Now $T_{\tau_{k}} \mapsto T_{\tau_{k}}$ gives an isomorphism from an Iwahori-Hecke algebra (see 3.2) specialized at $v=\sqrt{q}$ to the algebra $H\left(\mathbf{G}^{F}, \mathcal{P}^{F}, \mathbf{E}\right)$. The function $k \mapsto N_{k}$ coincides with the function $k \mapsto L\left(\tau_{k}\right)$ in 25.2.

(The results in this subsection appeared in [L10,L11]. In the special case where $\mathcal{P}=\mathcal{B}, u=1$ and $\mathbf{E}$ is the trivial vector bundle $\mathbf{C}$, they were first proved by Iwahori-Matsumoto [IM]; in this case, $N_{k}=1$ for all $k$.)

0.7. Let $V$ be an $\mathbf{F}_{q}((\epsilon))$-vector space of dimension $n \in[2, \infty)$ with a fixed volume form $\omega$. Then $G=S L\left(\mathbf{F} \otimes_{\mathbf{F}_{q}} V\right)$ is as in 0.6. Let $\mathfrak{A}=\mathbf{F}_{q}[[\epsilon]]$. A lattice in $V$ is an $\mathfrak{A}$-submodule of $V$ of rank $n$ which generates $V$. For a lattice $\mathcal{L}$ in $V$ we set $\operatorname{vol}(\mathcal{L})=r$ where $m \in \mathbf{Z}$ is defined by the condition that the $n$-th exterior power of $\mathcal{L}$ (an $\mathfrak{A}$-module) has $\epsilon^{-r} \omega$ as basis element. Let $\mathcal{F}$ be the set of all sequences of lattices $\left(\mathcal{L}_{j}\right)_{j \in \mathbf{Z}}$ such that

$$
\mathcal{L}_{j-1} \subset \mathcal{L}_{j}, \operatorname{vol}\left(\mathcal{L}_{j}\right)=j, \epsilon \mathcal{L}_{j}=\mathcal{L}_{j-n}
$$


for all $j$. We may identify $\mathcal{B}^{F}$ with $\mathcal{F}$ as sets with (transitive) $G^{F}$-action.

Let $\mathcal{L}_{*}=\left(\mathcal{L}_{j}\right)_{j \in \mathbf{Z}}, \mathcal{L}_{*}^{\prime}=\left(\mathcal{L}_{j}^{\prime}\right)_{j \in \mathbf{Z}}$ be elements of $\mathcal{F}$. For $i, j \in \mathbf{Z}$ we set

$$
d_{i j}=\operatorname{dim} \frac{\mathcal{L}_{i}^{\prime} \cap \mathcal{L}_{j}}{\mathcal{L}_{i}^{\prime} \cap \mathcal{L}_{j-1}} \in\{0,1\}
$$

For $i \in \mathbf{Z}$ let $X_{i}=\left\{j \in \mathbf{Z} ; d_{i j}=1\right\}$. Then $X_{i-1} \subset X_{i}$ for all $i$ and $\sharp\left(X_{i}-X_{i-1}\right)=1$ for all $i$. Define $a_{i} \in \mathbf{Z}$ by $X_{i}=X_{i-1} \sqcup\left\{a_{i}\right\}$. Now $X_{i-n}=X_{i}-n$. Hence

$$
a_{i+n}=a_{i}+n \text { for all } i \in \mathbf{Z} \text {. }
$$

One can check that $i \mapsto a_{i}$ is a bijection $\mathbf{Z} \rightarrow \mathbf{Z}$. Using the fact that $\operatorname{vol}\left(\mathcal{L}_{j}\right)=$ $\operatorname{vol}\left(\mathcal{L}_{j}^{\prime}\right)=j$ we see that

$$
\sum_{i=1}^{n}\left(a_{i}-i\right)=0 .
$$

This gives a bijection of $W$ onto the group of all bijections $\mathbf{Z} \stackrel{\sim}{\longrightarrow} \mathbf{Z}$ that satisfy (a),(b) (see 1.12).

0.8. Let $V, n$ be as in 0.7 . Assume that $n=2 m$ and that $V$ has a fixed nondegenerate symplectic form $\langle\rangle:, V \times V \rightarrow \mathbf{F}_{q}((\epsilon))$. Then $G=S p\left(\mathbf{F} \otimes_{\mathbf{F}_{q}} V\right)$ is as in 0.6. If $\mathcal{L}$ is a lattice in $V$ then $\mathcal{L}^{\sharp}=\{x \in V ;\langle x, \mathcal{L}\rangle \in \mathfrak{A}\}$ is again a lattice; moreover, $\left(\mathcal{L}^{\sharp}\right)^{\sharp}=\mathcal{L}$. Assume that $m=r+k^{2}+k+l^{2}+l$ where $k, l, r \in \mathbf{N}, r \geq 1$. Let $\mathcal{N}$ be the set of all integers of the form $a+2 m t$ where $t \in \mathbf{Z}$ and

$$
k^{2}+k \leq a \leq k^{2}+k+r \text { or }-\left(k^{2}+k+r\right) \leq a \leq-\left(k^{2}+k\right) .
$$

Let $\mathcal{F}$ be the set of all sequences of lattices $\left(\mathcal{L}_{j}\right)_{j \in \mathcal{N}}$ such that

$\mathcal{L}_{j} \subset \mathcal{L}_{j^{\prime}}$ if $j \leq j^{\prime}$ in $\mathcal{N}$

$\mathcal{L}_{j}^{\sharp}=\mathcal{L}_{-j}$ for all $j \in \mathcal{N}$,

$\epsilon \mathcal{L}_{j}=\mathcal{L}_{j-2 m}$ for all $j \in \mathcal{N}$,

$\operatorname{vol}\left(\mathcal{L}_{j}\right)=j$ for all $j \in \mathcal{N}$.

Here the volume of a lattice is defined in terms of the volume form on $V$ attached to the symplectic form. There is a unique $G$-conjugacy class $\mathcal{P}$ of parahoric subgroups of $G$ such that, if $\left(\mathcal{L}_{j}\right)_{j \in \mathcal{N}} \in \mathcal{F}$, then

$$
\left\{g \in G ; g\left(\mathbf{F} \otimes \mathcal{L}_{j}\right)=\mathbf{F} \otimes \mathcal{L}_{j} \quad \forall j \in \mathcal{N}\right\} \in \mathcal{P} .
$$

We may identify $\mathcal{P}^{F}$ and $\mathcal{F}$ as sets with $G^{F}$-action. If $\left(\mathcal{L}_{j}\right)_{j \in \mathcal{N}} \in \mathcal{F}$, then the $\mathbf{F}_{q}$-vector space $\mathcal{L}_{k^{2}+k} / \mathcal{L}_{-k^{2}-k}$ (of dimension $2 k^{2}+2 k$ ) has a natural nondegenerate symplectic form induced by $x, y \mapsto R e s\langle x, y\rangle$ and the $\mathbf{F}_{q}$-vector space $\mathcal{L}_{2 m-k^{2}-k-r} / \mathcal{L}_{k^{2}+k+r}$ (of dimension $2 l^{2}+2 l$ ) has a natural non-degenerate symplectic form induced by $x, y \mapsto \operatorname{Res}\langle x, \epsilon y\rangle$. Here Res $: \mathbf{F}_{q}((\epsilon)) \rightarrow \mathbf{F}_{q}$ denotes residue at 0 . 
Let $\mathcal{D}_{k}$ be a functor as in 0.5 and let $\mathcal{D}_{l}$ be an analogous functor obtained by replacing $k$ by $l$. Let $\mathbf{E}$ be the vector bundle over $\mathcal{P}^{F}$ (or equivalently $\mathcal{F}$ ) whose fibre at $\left(\mathcal{L}_{j}\right)_{j \in \mathcal{N}} \in \mathcal{F}$ is

$$
\mathcal{D}_{k}\left(\mathcal{L}_{k^{2}+k} / \mathcal{L}_{-k^{2}-k}\right) \otimes \mathcal{D}_{l}\left(\mathcal{L}_{2 m-k^{2}-k-r} / \mathcal{L}_{k^{2}+k+r}\right) .
$$

This vector bundle is naturally $G^{F}$-equivariant (since $\mathcal{D}_{k}, \mathcal{D}_{l}$ are functors). Hence $H\left(G^{F}, \mathcal{P}^{F}, \mathbf{E}\right)$ is defined. In our case, the Iwahori-Hecke algebra corresponds to the weighted Coxeter graph

$$
\bullet_{2 k+1}=\bullet_{1}-\bullet_{1}-\cdots-\bullet_{1}=\bullet_{2 l+1}
$$

$(r+1$ vertices); in the case where $r=1$ this should be interpreted as a Coxeter graph with 2 vertices marked by $2 k+1,2 l+1$, joined by a quadruple edge.

0.9. Notation. We set $[a, b]=\{z \in \mathbf{Z} ; a \leq z \leq b\},[a, b)=\{z \in \mathbf{Z} ; a \leq z<b\}$. If $X$ is a subset of a group $G$, we denote by $\langle X\rangle$ the subgroup of $G$ generated by $X$.

\section{Contents}

1. Coxeter groups

2. Partial order on $W$

3. The algebra $\mathcal{H}$

4. The bar operator

5. The elements $c_{w}$

6. Left or right multiplication by $c_{s}$

7. Dihedral groups

8. Cells

9. Cosets of parabolic subgroups

10. Inversion

11. The longest element for a finite $W$

12. Examples of elements $D_{w}$

13. The function $\mathbf{a}$

14. Conjectures

15. Example: the split case

16. Example: the quasisplit case

17. Example: the infinite dihedral case

18. The ring $J$

19. Algebras with trace form

20. The function $\mathbf{a}_{E}$

21. Study of a left cell

22. Constructible representations

23. Two-sided cells

24. Virtual cells

25. Relative Coxeter groups

26. Representations

27. A new realization of Hecke algebras 


\section{Coxeter groups}

1.1. Let $S$ be a finite set and let $\left(m_{s, s^{\prime}}\right)_{\left(s, s^{\prime}\right) \in S \times S}$ be a matrix with entries in $\mathbf{N} \cup\{\infty\}$ such that $m_{s, s}=1$ for all $s$ and $m_{s, s^{\prime}}=m_{s^{\prime}, s} \geq 2$ for all $s \neq s^{\prime}$. (A Coxeter matrix.) In the case where $m_{s, s^{\prime}} \in\{2,3,4,6, \infty\}$ for all $s \neq s^{\prime}$, the matrix $\left(m_{s, s^{\prime}}\right)_{\left(s, s^{\prime}\right) \in S \times S}$ is completely described by a graph (the Coxeter graph) with set of vertices in bijection with $S$ where the vertices corresponding to $s \neq s^{\prime}$ are joined by an edge if $m_{s, s^{\prime}}=3$, by a double edge if $m_{s, s^{\prime}}=4$, by a triple edge if $m_{s, s^{\prime}}=6$, by a quadruple edge if $m_{s, s^{\prime}}=\infty$.

Let $W$ be the group defined by the generators $s(s \in S)$ and relations

$$
\left(s s^{\prime}\right)^{m_{s, s^{\prime}}}=1
$$

for any $s, s^{\prime}$ in $S$ such that $m_{s, s^{\prime}}<\infty$. We say that $W, S$ is a Coxeter group. Note that the Coxeter matrix is uniquely determined by $W, S$ (see 1.3(b) below). We sometimes refer to $W$ itself as a Coxeter group. In $W$ we have $s^{2}=1$ for all $s$. Clearly, there is a unique homomorphism

$$
\operatorname{sgn}: W \rightarrow\{1,-1\}
$$

such that $\operatorname{sgn}(s)=-1$ for all $s$. ("Sign representation".)

For $w \in W$ let $l(w)$ be the smallest integer $q \geq 0$ such that $w=s_{1} s_{2} \ldots s_{q}$ with $s_{1}, s_{2}, \ldots, s_{q}$ in $S$. (We then say that $w=s_{1} s_{2} \ldots s_{q}$ is a reduced expression and $l(w)$ is the length of $w$.) Now $l(1)=0, l(s)=1$ for $s \in S$. (Indeed, $s \neq 1$ in $W$ since $\operatorname{sgn}(s)=-1, \operatorname{sgn}(1)=1$.)

Lemma 1.2. Let $w \in W, s \in S$.

(a) We have either $l(s w)=l(w)+1$ or $l(s w)=l(w)-1$.

(b) We have either $l(w s)=l(w)+1$ or $l(w s)=l(w)-1$.

Clearly, $\operatorname{sgn}(w)=(-1)^{l(w)}$. Since $\operatorname{sgn}(s w)=-\operatorname{sgn}(w)$, we have $(-1)^{l(s w)}=$ $-(-1)^{l(w)}$. Hence $l(s w) \neq l(w)$. This, together with the obvious inequalities $l(w)-1 \leq l(s w) \leq l(w)+1$ gives (a). The proof of (b) is similar.

Proposition 1.3. Let $E$ be an $\mathbf{R}$-vector space with basis $\left(e_{s}\right)_{s \in S}$. For $s \in S$ define a linear map $\sigma_{s}: E \rightarrow E$ by $\sigma_{s}\left(e_{s^{\prime}}\right)=e_{s^{\prime}}+2 \cos \frac{\pi}{m_{s, s^{\prime}}} e_{s}$ for all $s^{\prime} \in S$.

(a) There is a unique homomorphism $\sigma: W \rightarrow G L(E)$ such that $\sigma(s)=\sigma_{s}$ for all $s \in S$.

(b) If $s \neq s^{\prime}$ in $S$, then $s s^{\prime}$ has order $m_{s, s^{\prime}}$ in $W$. In particular, $s \neq s^{\prime}$ in $W$.

We have $\sigma_{s}\left(e_{s}\right)=-e_{s}$ and $\sigma_{s}$ induces the identity map on $E / \mathbf{R} e_{s}$. Hence $\sigma_{s}^{2}=1$. Now let $s \neq s^{\prime}$ in $S, m=m_{s, s^{\prime}}, \Phi=\sigma_{s} \sigma_{s^{\prime}}$. We have

$\Phi\left(e_{s}\right)=\left(4 \cos ^{2} \frac{\pi}{m}-1\right) e_{s}+2 \cos \frac{\pi}{m} e_{s^{\prime}}$,

$\Phi\left(e_{s^{\prime}}\right)=-2 \cos \frac{\pi}{m} e_{s}-e_{s^{\prime}}$.

Hence $\Phi$ restricts to an endomorphism $\phi$ of $\mathbf{R} e_{s} \oplus \mathbf{R} e_{s^{\prime}}$ whose characteristic polynomial is

$$
X^{2}-2 \cos \frac{2 \pi}{m} X+1=\left(X-e^{2 \pi \sqrt{-1} / m}\right)\left(X-e^{-2 \pi \sqrt{-1} / m}\right) .
$$


It follows that, if $2<m<\infty$, then $1+\phi+\phi^{2}+\cdots+\phi^{m-1}=0$. The same holds if $m=2$ (in this case we see directly that $\phi=-1$ ). Since $\Phi$ induces the identity map on $E /\left(\mathbf{R} e_{s} \oplus \mathbf{R} e_{s^{\prime}}\right)$, it follows that $\Phi: E \rightarrow E$ has order $m$ (if $m<\infty$ ). If $m=\infty$, we have $\phi \neq 1$ and $(\phi-1)^{2}=0$, hence $\phi$ has infinite order and $\Phi$ has also infinite order. Now (a), (b) follow.

Corollary 1.4. Let $s_{1} \neq s_{2}$ in $S$. For $k \geq 0$ let $1_{k}=s_{1} s_{2} s_{1} \ldots$ ( $k$ factors), $2_{k}=s_{2} s_{1} s_{2} \ldots$ ( $k$ factors $)$.

(a) Assume that $m=m_{s_{1}, s_{2}}<\infty$. Then $\left\langle s_{1}, s_{2}\right\rangle$ consists of the elements $1_{k}, 2_{k}(k=0,1, \ldots, m)$; these elements are distinct except for the equalities $1_{0}=$ $2_{0}, 1_{m}=2_{m}$. For $k \in[0, m]$ we have $l\left(1_{k}\right)=l\left(2_{k}\right)=k$.

(b) Assume that $m_{s_{1}, s_{2}}=\infty$. Then $\left\langle s_{1}, s_{2}\right\rangle$ consists of the elements $1_{k}, 2_{k}$ $(k=0,1, \ldots)$; these elements are distinct except for the equality $1_{0}=2_{0}$. For all $k \geq 0$ we have $l\left(1_{k}\right)=l\left(2_{k}\right)=k$.

This follows immediately from $1.3(\mathrm{~b})$.

We identify $S$ with a subset of $W$ (see $1.3(\mathrm{~b}))$ said to be the set of simple reflections. Let

$$
T=\cup_{w \in W} w S w^{-1} \subset W .
$$

Proposition 1.5. Let $R=\{1,-1\} \times T$. For $s \in S$ define $U_{s}: R \rightarrow R$ by $U_{s}(\epsilon, t)=\left(\epsilon(-1)^{\delta_{s, t}}\right.$, sts) where $\delta$ is the Kronecker symbol. There is a unique homomorphism $U$ of $W$ into the group of permutations of $R$ such that $U(s)=U_{s}$ for all $s \in S$.

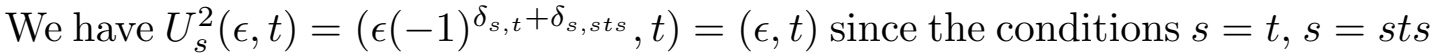
are equivalent. Thus, $U_{s}^{2}=1$. For $s \neq s^{\prime}$ in $S$ with $m=m_{s, s^{\prime}}<\infty$ we have

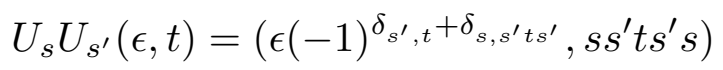

hence

$$
\left(U_{s} U_{s^{\prime}}\right)^{m}(\epsilon, t)=\left(\epsilon(-1)^{\kappa},\left(s s^{\prime}\right)^{m} t\left(s^{\prime} s\right)^{m}\right)=\left(\epsilon(-1)^{\kappa}, t\right)
$$

where

$$
\kappa=\delta_{s^{\prime}, t}+\delta_{s, s^{\prime} t s^{\prime}}+\delta_{s^{\prime}, s s^{\prime} t s^{\prime} s}+\cdots=\delta_{s^{\prime}, t}+\delta_{s^{\prime} s s^{\prime}, t}+\delta_{s^{\prime} s s^{\prime} s s^{\prime}, t}+\ldots
$$

(both sums have exactly $2 m$ terms). It is enough to show that $\kappa$ is even, or that $t$ appears an even number of times in the $2 m$-term sequence $s^{\prime}, s^{\prime} s s^{\prime}, s^{\prime} s s^{\prime} s s^{\prime}, \ldots$ This follows from the fact that in this sequence the $k$-th term is equal to the $(k+m)$-th term for $k=1,2, \ldots, m$.

Proposition 1.6. Let $w \in W$. Let $w=s_{1} s_{2} \ldots s_{q}$ be a reduced expression.

(a) The elements $s_{1}, s_{1} s_{2} s_{1}, s_{1} s_{2} s_{3} s_{2} s_{1}, \ldots, s_{1} s_{2} \ldots s_{q} \ldots s_{2} s_{1}$ are distinct. 
(b) These elements form a subset of $T$ that depends only on $w$, not on the choice of reduced expression for it.

Assume that $s_{1} s_{2} \ldots s_{i} \ldots s_{2} s_{1}=s_{1} s_{2} \ldots s_{j} \ldots s_{2} s_{1}$ for some $1 \leq i<j \leq q$. Then $s_{i}=s_{i+1} s_{i+2} \ldots s_{j} \ldots s_{i+2} s_{i+1}$ hence

$$
\begin{aligned}
s_{1} s_{2} \ldots s_{q} & =s_{1} s_{2} \ldots s_{i-1}\left(s_{i+1} s_{i+2} \ldots s_{j} \ldots s_{i+2} s_{i+1}\right) s_{i+1} \ldots s_{j} s_{j+1} \ldots s_{q} \\
& =s_{1} s_{2} \ldots s_{i-1} s_{i+1} s_{i+2} \ldots s_{j-1} s_{j+1} \ldots s_{q}
\end{aligned}
$$

which shows that $l(w) \leq q-2$, contradiction. This proves (a).

For $(\epsilon, t) \in R$ we have (see 1.5) $U\left(w^{-1}\right)(\epsilon, t)=\left(\epsilon \eta(w, t), w^{-1} t w\right)$ where $\eta(w, t)=$ \pm 1 depends only on $w, t$. On the other hand,

$$
\begin{aligned}
& U\left(w^{-1}\right)(\epsilon, t)=U_{s_{q}} \ldots U_{s_{1}}(\epsilon, t)
\end{aligned}
$$

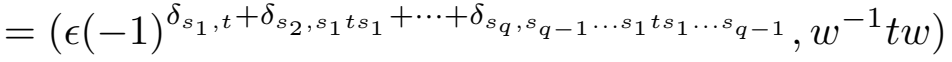

$$
\begin{aligned}
& =\left(\epsilon(-1)^{\delta_{s_{1}, t}+\delta_{s_{1} s_{2} s_{1}, t}+\cdots+\delta_{s_{1} \ldots s_{q} \ldots s_{1}, t}}, w^{-1} t w\right) \text {. }
\end{aligned}
$$

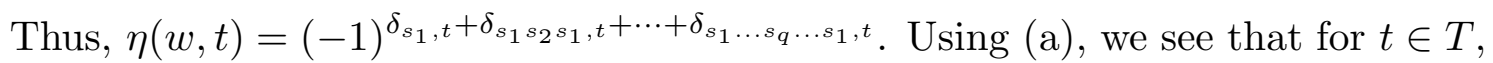
the sum $\delta_{s_{1}, t}+\delta_{s_{1} s_{2} s_{1}, t}+\cdots+\delta_{s_{1} \ldots s_{q} \ldots s_{1}, t}$ is 1 if $t$ belongs to the subset in (b) and is 0 , otherwise. Hence the subset in (b) is just $\{t \in T ; \eta(w, t)=-1\}$. This completes the proof.

Proposition 1.7. Let $w \in W, s \in S$ be such that $l(s w)=l(w)-1$. Let $w=$ $s_{1} s_{2} \ldots s_{q}$ be a reduced expression. Then there exists $j \in[1, q]$ such that

$s s_{1} s_{2} \ldots s_{j-1}=s_{1} s_{2} \ldots s_{j}$.

Let $w^{\prime}=s w$. Let $w^{\prime}=s_{1}^{\prime} s_{2}^{\prime} \ldots s_{q-1}^{\prime}$ be a reduced expression. Then $w=$ $s s_{1}^{\prime} s_{2}^{\prime} \ldots s_{q-1}^{\prime}$ is another reduced expression. By 1.6(b), the $q$-term sequences

$$
s_{1}, s_{1} s_{2} s_{1}, s_{1} s_{2} s_{3} s_{2} s_{1}, \ldots \text { and } s, s s_{1}^{\prime} s, s s_{1}^{\prime} s_{2}^{\prime} s_{1}^{\prime} s, \ldots
$$

coincide up to rearranging terms. In particular, $s=s_{1} s_{2} \ldots s_{j} \ldots s_{2} s_{1}$ for some $j \in[1, q]$. The proposition follows.

1.8. Let $X$ be the set of all sequences $\left(s_{1}, s_{2}, \ldots, s_{q}\right)$ in $W$ such that $s_{1} s_{2} \ldots s_{q}$ is a reduced expression in $W$. We regard $X$ as the vertices of a graph in which $\left(s_{1}, s_{2}, \ldots, s_{q}\right),\left(s_{1}^{\prime}, s_{2}^{\prime}, \ldots, s_{q^{\prime}}^{\prime}\right)$ are joined if one is obtained from the other by replacing $m$ consecutive entries of form $s, s^{\prime}, s, s^{\prime}, \ldots$ by the $m$ entries $s^{\prime}, s, s^{\prime}, s, \ldots$; here $s \neq s^{\prime}$ in $S$ are such that $m=m_{s, s^{\prime}}<\infty$. We use the notation

$$
\left(s_{1}, s_{2}, \ldots, s_{q}\right) \sim\left(s_{1}^{\prime}, s_{2}^{\prime}, \ldots, s_{q^{\prime}}^{\prime}\right)
$$

for " $\left(s_{1}, s_{2}, \ldots, s_{q}\right),\left(s_{1}^{\prime}, s_{2}^{\prime}, \ldots, s_{q^{\prime}}^{\prime}\right)$ are in the same connected component of $X "$. (When this holds we have necessarily $q=q^{\prime}$ and $s_{1} s_{2} \ldots s_{q}=s_{1}^{\prime} s_{2}^{\prime} \ldots s_{q}^{\prime}$ in $W$.) 
The following result is due to Matsumoto and Tits.

Theorem 1.9. Let $\mathbf{s}=\left(s_{1}, s_{2}, \ldots, s_{q}\right), \mathbf{s}^{\prime}=\left(s_{1}^{\prime}, s_{2}^{\prime}, \ldots, s_{q}^{\prime}\right)$ in $X$ be such that $s_{1} s_{2} \ldots s_{q}=s_{1}^{\prime} s_{2}^{\prime} \ldots s_{q}^{\prime}=w \in W$. Then $\mathbf{s} \sim \mathbf{s}^{\prime}$.

Let $C$ (resp. $C^{\prime}$ ) be the connected component of $X$ that contains $\mathbf{s}$ (resp. $\mathbf{s}^{\prime}$ ). For $i \in[1, q]$ we set

$\mathbf{s}(i)=\left(\ldots, s_{1}^{\prime}, s_{1}, s_{1}^{\prime}, s_{1}, s_{2}, s_{3}, \ldots, s_{i}\right)$ (a $q$-element sequence in $S$ ),

$\underline{\mathbf{s}}(i)=\ldots s_{1}^{\prime} s_{1} s_{1}^{\prime} s_{1} s_{2} s_{3} \ldots s_{i} \in W$ (the product of this sequence).

Let $C(i)$ be the connected component of $X$ that contains $\mathbf{s}(i)$. Then $\mathbf{s}=\mathbf{s}(q)$. Hence $C=C(q)$.

We argue by induction on $q$. The theorem is obvious for $q \in\{0,1\}$. We now assume that $q \geq 2$ and that the theorem is known for $q-1$. We first prove the following weaker statement.

(A) In the setup of the theorem we have either $\mathbf{s} \sim \mathbf{s}^{\prime}$ or

$$
s_{1} s_{2} \ldots s_{q}=s_{1}^{\prime} s_{1} s_{2} \ldots s_{q-1} \text { and }\left(s_{1}^{\prime}, s_{1}, s_{2}, \ldots, s_{q-1}\right) \sim\left(s_{1}^{\prime}, s_{2}^{\prime}, \ldots, s_{q}^{\prime}\right) .
$$

We have $l\left(s_{1}^{\prime} w\right)=l(w)-1$. By 1.7 we have $s_{1}^{\prime} s_{1} s_{2} \ldots s_{i-1}=s_{1} s_{2} \ldots s_{i}$ for some $i \in[1, q]$, so that $w=s_{1}^{\prime} s_{1} s_{2} \ldots s_{i-1} s_{i+1} \ldots s_{q}$. In particular,

$$
\left(s_{1}^{\prime}, s_{1}, s_{2}, \ldots, s_{i-1}, s_{i+1}, \ldots, s_{q}\right) \in X \text {. }
$$

By the induction hypothesis, $\left(s_{1}, s_{2}, \ldots, s_{i-1}, s_{i+1}, \ldots, s_{q}\right) \sim\left(s_{2}^{\prime}, \ldots, s_{q}^{\prime}\right)$. Hence

$$
\left(s_{1}^{\prime}, s_{1}, s_{2}, \ldots, s_{i-1}, s_{i+1}, \ldots, s_{q}\right) \sim\left(s_{1}^{\prime}, s_{2}^{\prime}, \ldots, s_{q}^{\prime}\right)
$$

Assume first that $i<q$. Then from $s_{1}^{\prime} s_{1} s_{2} \ldots s_{i-1} s_{i+1} \ldots s_{q-1}=s_{1} s_{2} \ldots s_{q-1}$ and the induction hypothesis we deduce that

$\left(s_{1}^{\prime}, s_{1}, s_{2}, \ldots, s_{i-1}, s_{i+1}, \ldots, s_{q-1}\right) \sim\left(s_{1}, s_{2}, \ldots, s_{q-1}\right)$, hence

$\left(s_{1}^{\prime}, s_{1}, s_{2}, \ldots, s_{i-1}, s_{i+1}, \ldots, s_{q-1}, s_{q}\right) \in C$.

Combining this with (b) we deduce that $C=C^{\prime}$.

Assume next that $i=q$ so that $s_{1} s_{2} \ldots s_{q}=s_{1}^{\prime} s_{1} s_{2} \ldots s_{q-1}$. Then (b) shows that (a) holds. Thus, $(A)$ is proved.

Next we prove for $p \in[0, q-2]$ the following generalization of $(A)$.

$\left(A_{p}^{\prime}\right)$ In the setup of the theorem we have either $C=C^{\prime}$ or:

for $i \in[q-p-1, q]$ we have $\mathbf{s}(i) \in X, \underline{\mathbf{s}}(i)=w, C_{i}=C$ if $i-q \in 2 \mathbf{Z}, C_{i}=C^{\prime}$ if $i-q \notin 2 \mathbf{Z}$.

For $p=0$ this reduces to $(A)$. Assume now that $p>0$ and that $\left(A_{p-1}^{\prime}\right)$ is already known. We prove that $\left(A_{p}^{\prime}\right)$ holds.

If $C=C^{\prime}$, then we are done. Hence by $\left(A_{p-1}^{\prime}\right)$ we may assume that: for $i \in[q-p, q]$ we have $\mathbf{s}(i) \in X, \underline{\mathbf{s}}(i)=w, C_{i}=C$ if $i-q \in 2 \mathbf{Z}, C_{i}=C^{\prime}$ if $i-q \notin 2 \mathbf{Z}$.

Applying $(A)$ to $\mathbf{s}(q-p), \mathbf{s}\left(q-p+1\right.$ ) (instead of $\left.\mathbf{s}, \mathbf{s}^{\prime}\right)$, we see that either $C_{q-p}=C_{q-p+1}$ or: 
$\mathbf{s}(q-p), \mathbf{s}(q-p-1)$ are in $X, \underline{\mathbf{s}}(q-p)=\underline{\mathbf{s}}(q-p-1)$ and $C_{q-p-1}=C_{q-p+1}$. In both cases, we see that $\left(A_{p}^{\prime}\right)$ holds.

This completes the inductive proof of $\left(A_{p}^{\prime}\right)$. In particular, $\left(A_{q-2}^{\prime}\right)$ holds. In other words, in the setup of the theorem, either $C=C^{\prime}$ holds or:

(c) for $i \in[1, q]$ we have $\mathbf{s}(i) \in X, \underline{\mathbf{s}}(i)=w, C_{i}=C$ if $i-q \in 2 \mathbf{Z}, C_{i}=C^{\prime}$ if $i-q \notin 2 \mathbf{Z}$.

If $C=C^{\prime}$, then we are done. Hence we may assume that (c) holds. In particular,

$$
\mathbf{s}(2) \in X, \mathbf{s}(1) \in X, \underline{\mathbf{s}}(2)=\underline{\mathbf{s}}(1) \text {. }
$$

From $\mathbf{s}(1) \in X$ and $q \geq 2$ we see that $s^{\prime} \neq s$ and that $q \leq m=m_{s, s^{\prime}}$. From $\underline{\mathbf{s}}(2)=\underline{\mathbf{s}}(1)$ we see that $s_{2} \in\left\langle s_{1}, s_{1}^{\prime}\right\rangle$, hence $s_{2}$ is either $s_{1}$ or $s_{1}^{\prime}$. In fact we cannot have $s_{2}=s_{1}$ since this would contradict $\mathbf{s}(2) \in X$. Hence $s_{2}=s_{1}^{\prime}$. We see that $\mathbf{s}(2)=\left(\ldots, s_{1}^{\prime}, s_{1}, s_{1}^{\prime}, s_{1}, s_{1}^{\prime}\right)$ (the number of terms is $\left.q, q \leq m\right)$. Since $\underline{\mathbf{s}}(2)=\underline{\mathbf{s}}(1)$, it follows that $q=m$, so that $\mathbf{s}(2), \mathbf{s}(1)$ are joined in $X$. It follows that $C_{2}=C_{1}$. By (c), for some permutation $a, b$ of 1,2 we have $C_{a}=C, C_{b}=C^{\prime}$. Since $C_{a}=C_{b}$ it follows that $C=C^{\prime}$. The theorem is proved.

Proposition 1.10. Let $w \in W$ and let $s, t \in S$ be such that $l(s w t)=l(w), l(s w)=$ $l(w t)$. Then $s w=w t$.

Let $w=s_{1} s_{2} \ldots s_{q}$ be a reduced expression.

Assume first that $l(w t)=q+1$. Then $s_{1} s_{2} \ldots s_{q} t$ is a reduced expression for $w t$. Now $l(s w t)=l(w t)-1$ hence by 1.7 there exists $i \in[1, q]$ such that $s s_{1} s_{2} \ldots s_{i-1}=$ $s_{1} s_{2} \ldots s_{i}$ or else $s s_{1} s_{2} \ldots s_{q}=s_{1} s_{2} \ldots s_{q} t$. If the second alternative occurs, we are done. If the first alternative occurs, we have $s w=s_{1} s_{2} \ldots s_{i-1} s_{i+1} \ldots s_{q}$ hence $l(s w) \leq q-1$. This contradicts $l(s w)=l(w t)$.

Assume next that $l(w t)=q-1$. Let $w^{\prime}=w t$. Then $l\left(s w^{\prime} t\right)=l\left(w^{\prime}\right), l\left(s w^{\prime}\right)=$ $l\left(w^{\prime} t\right)$. We have $l\left(w^{\prime} t\right)=l\left(w^{\prime}\right)+1$ hence the first part of the proof applies and gives $s w^{\prime}=w^{\prime} t$. Hence $s w=w t$. The proposition is proved.

1.11. We can regard $S$ as the set of vertices of a graph in which $s, s^{\prime}$ are joined if $m_{s, s^{\prime}}>2$. We say that $W$ is irreducible if this graph is connected. It is easy to see that in general, $W$ is naturally a product of irreducible Coxeter groups, corresponding to the connected components of $S$.

In the setup of 1.3, let (, ) $E \times E \rightarrow \mathbf{R}$ be the symmetric $\mathbf{R}$-bilinear form given by $\left(e_{s}, e_{s^{\prime}}\right)=-\cos \frac{\pi}{m_{s, s^{\prime}}}$. Then $\sigma(w): E \rightarrow E$ preserves $($,$) for any w \in W$.

We say that $W$ is tame if $(e, e) \geq 0$ for any $e \in E$. It is easy to see that, if $W$ is finite then $W$ is tame.

We say that $W$ is integral if, for any $s \neq s^{\prime}$ in $S$, we have $4 \cos ^{2} \frac{\pi}{m_{s, s^{\prime}}} \in \mathbf{N}$ (or equivalently $\left.m_{s, s^{\prime}} \in\{2,3,4,6, \infty\}\right)$.

We will be mainly interested in the case where $W$ is tame. The tame, irreducible $W$ are of three kinds:

(a) finite, integral;

(b) finite, non-integral;

(c) tame, infinite (and automatically integral). 
1.12. For $k \in \mathbf{Z}$ define $\rho_{k}: \mathbf{Z} \rightarrow \mathbf{Z}$ by $\rho_{k}(z)=z+k$. Let $n \geq 2$. Let $\tilde{W}$ be the group of all permutations $\sigma: \mathbf{Z} \rightarrow \mathbf{Z}$ such that $\sigma \rho_{n}=\rho_{n} \sigma$. Define $\chi: \tilde{W} \rightarrow \mathbf{Z}$ by $\chi(\sigma)=\sum_{k \in X}(\sigma(k)-k)$ where $X$ is a set of representatives for the residue classes $\bmod n$ in $\mathbf{Z}$. One checks that $\chi$ does not depend on the choice of $X$ and $\chi$ is a group homomorphism with image $n \mathbf{Z}$. Now $\tilde{W}^{\prime}=\operatorname{ker}(\chi)$ is generated by $\left\{s_{m} ; m \in \mathbf{Z} / n \mathbf{Z}\right\}$ where $s_{m}: \mathbf{Z} \rightarrow \mathbf{Z}$ is defined by

$s_{m}(z)=z+1$ if $z=m \bmod n$,

$s_{m}(z)=z-1$ if $z=m+1 \bmod n$,

$s_{m}(z)=z$ for all other $z \in \mathbf{Z}$.

It is a Coxeter group on these generators, said to be of type $\tilde{A}_{n-1}$. (This description of $\tilde{W}^{\prime}$ appears in [L4].) For $n \geq 3, m, m^{\prime} \in \mathbf{Z} / n \mathbf{Z}$ are joined by a single edge in the Coxeter graph if $m-m^{\prime}=1 \bmod n$ and are not joined otherwise. For $n=2$, $0,1 \in \mathbf{Z} / 2 \mathbf{Z}$ are joined by a quadruple edge in the Coxeter graph. The length function on $\tilde{W}^{\prime}$ is given by

$$
l(\sigma)=\sharp\left(Y_{\sigma} / \tau_{n}\right)
$$

where, for $\sigma \in \tilde{W}^{\prime}$,

$$
Y_{\sigma}=\{(i, j) \in \mathbf{Z} \times \mathbf{Z} ; i<j, \sigma(i)>\sigma(j)\}
$$

and $Y_{\sigma} / \tau_{n}$ is the (finite) set of orbits of $\tau_{n}: Y_{\sigma} \rightarrow Y_{\sigma},(i, j) \mapsto(i+n, j+n)$.

1.13. Assume now that $n=2 p \geq 4$, where $p \in \mathbf{N}$. Let $W$ be the subgroup of $\tilde{W}$ consisting of all $\sigma \in \tilde{W}$ that commute with the involution $\mathbf{Z} \rightarrow \mathbf{Z}, z \mapsto 1-z$. We compute $\chi(\sigma)$ for $\sigma \in W$, taking $X=\{-(p-1), \ldots,-1,0,1,2, \ldots, p\}$ :

$$
\begin{aligned}
& \chi(\sigma)=\sum_{k \in[1, p]}(\sigma(k)-k)+\sum_{k \in[1, p]}(\sigma(1-k)-(1-k)) \\
& =\sum_{k \in[1, p]}(\sigma(k)-k)+\sum_{k \in[1, p]}(1-\sigma(k)-(1-k))=0 .
\end{aligned}
$$

Thus, $W$ is a subgroup of $\tilde{W}^{\prime}$. Now $W$ is generated by $s_{0}^{\prime}, s_{1}^{\prime}, \ldots, s_{p}^{\prime}$ where

$$
s_{0}^{\prime}=s_{0}, s_{1}^{\prime}=s_{1} s_{-1}, s_{2}^{\prime}=s_{2} s_{-2}, \ldots, s_{p-1}^{\prime}=s_{p-1} s_{1-p}, s_{p}^{\prime}=s_{p} .
$$

It is a Coxeter group on these generators, said to be of type $\tilde{C}_{p}$. The Coxeter graph is

$$
\bullet=\bullet-\bullet-\ldots-\bullet=\bullet
$$

with vertices corresponding to $0,1,2, \ldots, p-1, p$.

Let $\sigma \in W$. We have a partition $Y_{\sigma}=Y_{\sigma}^{0} \sqcup Y_{\sigma}^{1}$ where

$$
Y_{\sigma}^{0}=\{(i, j) \in \mathbf{Z} \times \mathbf{Z} ; i<j, \sigma(i)>\sigma(j), i+j \neq 1 \quad \bmod 2 p\}
$$




$$
Y_{\sigma}^{1}=\{(i, j) \in \mathbf{Z} \times \mathbf{Z} ; i<j, \sigma(i)>\sigma(j), i+j=1 \quad \bmod 2 p\} .
$$

Now $Y_{\sigma}^{1} / \tau_{n}$ is the fixed point set of the involution of $Y_{\sigma} / \tau_{n}$ induced by the involution $(i, j) \mapsto(1-j, 1-i)$ of $Y_{\sigma}$. Hence we have $\sharp\left(Y_{\sigma} / \tau_{n}\right)=2 l^{0}(\sigma)+l^{1}(\sigma)$ where $l^{0}(\sigma)=\sharp\left(Y_{\sigma}^{0} / \tau_{n}\right) / 2, l^{1}(\sigma)=\sharp\left(Y_{\sigma}^{1} / \tau_{n}\right)$ are integers. Now $(i, j) \mapsto\left(i, \frac{i+j-1}{2 p}\right)$ is a bijection of $Y_{\sigma}^{1}$ with

$$
\{(i, h) \in \mathbf{Z} \times \mathbf{Z} ; 2 i<1+2 p h, 2 \sigma(i)>1+2 p h\}=\{(i, h) \in \mathbf{Z} \times \mathbf{Z} ; i \leq p h<\sigma(i)\}
$$

It follows that

$$
l^{1}(\sigma)=\sum_{i \in[1-p, p] ; i<\sigma(i)} f(i)
$$

where

$$
f(i)=\sharp(x \in p \mathbf{Z} ; i \leq x<\sigma(i)) .
$$

Let $\mathbf{Z}^{\prime}=[1, p]+2 p \mathbf{Z}, \mathbf{Z}^{\prime \prime}=[1-p, 0]+2 p \mathbf{Z}$; then $\mathbf{Z}=\mathbf{Z}^{\prime} \sqcup \mathbf{Z}^{\prime \prime}$. We have $l^{1}(\sigma)=$ $l^{\prime}(\sigma)+l^{\prime \prime}(\sigma)$ where

$$
\begin{aligned}
& l^{\prime}(\sigma)=\sum_{i \in[1-p, 0] ; \sigma(i) \in \mathbf{Z}^{\prime \prime}, i<\sigma(i)} \frac{f(i)}{2}+\sum_{i \in[1, p] ; \sigma(i) \in \mathbf{Z}^{\prime}, i<\sigma(i)} \frac{f(i)}{2} \\
& +\sum_{i \in[1-p, 0] ; \sigma(i) \in \mathbf{Z}^{\prime}, i<\sigma(i)} \frac{f(i)+1}{2}+\sum_{i \in[1, p] ; \sigma(i) \in \mathbf{Z}^{\prime \prime}, i<\sigma(i)} \frac{f(i)-1}{2}, \\
& l^{\prime \prime}(\sigma)=\sum_{i \in[1-p, 0] ; \sigma(i) \in \mathbf{Z}^{\prime \prime}, i<\sigma(i)} \frac{f(i)}{2}+\sum_{i \in[1, p] ; \sigma(i) \in \mathbf{Z}^{\prime}, i<\sigma(i)} \frac{f(i)}{2} \\
& +\sum_{i \in[1-p, 0] ; \sigma(i) \in \mathbf{Z}^{\prime}, i<\sigma(i)} \frac{f(i)-1}{2}+\sum_{i \in[1, p] ; \sigma(i) \in \mathbf{Z}^{\prime \prime}, i<\sigma(i)} \frac{f(i)+1}{2} .
\end{aligned}
$$

These are integers since $f(i)$ is even if $i, \sigma(i)$ are in the same set $\mathbf{Z}^{\prime}$ or $\mathbf{Z}^{\prime \prime}$ and is odd otherwise. We see that the length of $\sigma$ in $\tilde{W}^{\prime}$ is $2 l^{0}(\sigma)+l^{\prime}(\sigma)+l^{\prime \prime}(\sigma)$. On the other hand, the length of $\sigma$ in $W$ is

$$
l^{0}(\sigma)+l^{\prime}(\sigma)+l^{\prime \prime}(\sigma)
$$

Now $l^{\prime}(\sigma)$ (resp. $l^{\prime \prime}(\sigma)$ ) is the number of times that $s_{0}^{\prime}$ (resp. $s_{p}^{\prime}$ ) appears in a reduced expression of $\sigma$ in $W$.

One can show that $l^{0}, l^{\prime}, l^{\prime \prime}$ are weight functions on $W$ in the sense of 3.1.

Define $\chi^{\prime}: W \rightarrow\{ \pm 1\}$ and $\chi^{\prime \prime}: W \rightarrow\{ \pm 1\}$ by $\chi^{\prime}(\sigma)=(-1)^{l^{\prime}(\sigma)}, \chi^{\prime \prime}(\sigma)=$ $(-1)^{l^{\prime \prime}(\sigma)}$. Then $\chi^{\prime}, \chi^{\prime \prime}$ are group homomorphisms. by

Assuming that $p \geq 3$, let $W^{\prime}=\operatorname{ker}\left(\chi^{\prime}\right)$. This is the subgroup of $W$ generated

$$
s_{0}^{\prime} s_{1}^{\prime} s_{0}^{\prime}, s_{1}^{\prime}, s_{2}^{\prime}, \ldots, s_{p-1}^{\prime}, s_{p}^{\prime}
$$


It is a Coxeter group on these generators, said to be of type $\tilde{B}_{p}$. The Coxeter graph has vertices $\tilde{1}, 1,2, \ldots,(p-1), p$ corresponding to $s_{0}^{\prime} s_{1}^{\prime} s_{0}^{\prime}, s_{1}^{\prime}, s_{2}^{\prime}, \ldots, s_{p-1}^{\prime}, s_{p}^{\prime}$ and edges

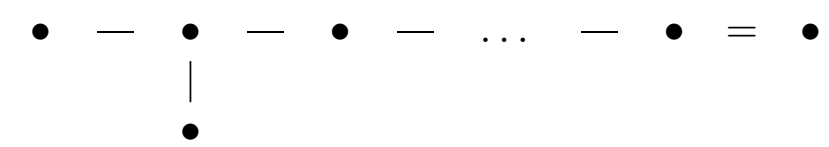

Assuming that $p \geq 4$, let $W^{\prime \prime}=\operatorname{ker}\left(\chi^{\prime}\right) \cap \operatorname{ker}\left(\chi^{\prime \prime}\right)$. This is the subgroup of $W$ (or $W^{\prime}$ ) generated by

$$
s_{0}^{\prime} s_{1}^{\prime} s_{0}^{\prime}, s_{1}^{\prime}, s_{2}^{\prime}, \ldots, s_{p-1}^{\prime}, s_{p}^{\prime} s_{p-1}^{\prime} s_{p}^{\prime} .
$$

It is a Coxeter group on these generators, said to be of type $\tilde{D}_{p}$. The Coxeter graph has vertices $\tilde{1}, 1,2, \ldots,(p-1), \widetilde{(p-1)}$ and edges

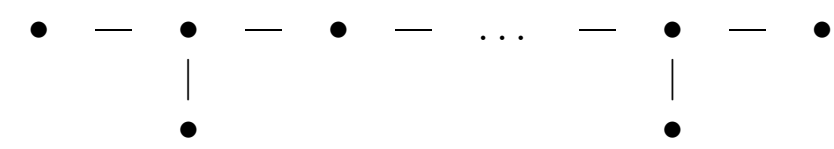

1.14. Let $p \geq q \geq r \geq 1$ be integers such that $p^{-1}+q^{-1}+r^{-1}=1$. Then $p, q, r$ is $3,3,3$ or $4,4,2$ or $6,3,2$. Thus, $q$ and $r$ divide $p$. Consider the graph with vertices

$$
\left\{\left(\frac{i p}{p}, 0,0\right) ; i \in[1, p]\right\} \cup\left\{\left(0, \frac{i p}{q}, 0\right) ; i \in[1, q]\right\} \cup\left\{\left(0,0, \frac{i p}{r}\right) ; i \in[1, r]\right\}
$$

where $(p, 0,0),(0, p, 0),(0,0, p)$ are identified; the edges are

$$
\begin{aligned}
& \left(\frac{i p}{p}, 0,0\right)-\left(\frac{(i+1) p}{p}, 0,0\right), 1 \leq i<p, \\
& \left(0, \frac{i p}{q}, 0\right)-\left(0, \frac{(i+1) p}{q}, 0\right), 1 \leq i<q, \\
& \left(0,0, \frac{i p}{r}\right)-\left(0,0, \frac{(i+1) p}{r}\right), 1 \leq i<r .
\end{aligned}
$$

The Coxeter group $W$ corresponding to this graph is said to be of type $\tilde{E}_{n}$ where $n=p+q+r-3$. Thus, $n \in\{6,7,8\}$.

Let $W$ be of type $\tilde{E}_{6}$. Let $W^{\prime}$ be the subgroup of $W$ generated by

$$
s_{1,0,0}, s_{2,0,0}, s_{3,0,0}, s_{0,2,0} s_{0,0,2}, s_{0,1,0} s_{0,0,1} \text {. }
$$

(The index of a generator of $W$ is the corresponding vertex of the graph.) Then $W^{\prime}$ is a Coxeter group on these generators, said to be of type $\tilde{F}_{4}$. The Coxeter graph is

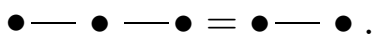

Let $W$ be of type $\tilde{D}_{4}$. The standard generators may be denoted by $s_{0}, s_{1}, s_{2}, s_{3}, s_{4}$ where the Coxeter graph has vertices $0,1,2,3,4$ with four edges joining 0 with $1,2,3,4$. Let $W^{\prime}$ be the subgroup of $W$ generated by $s_{1}, s_{0}, s_{2} s_{3} s_{4}$. Then $W^{\prime}$ is a Coxeter group on these generators, said to be of type $\tilde{G}_{2}$. The Coxeter graph is

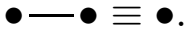


1.15. The collection of Coxeter groups of type $\tilde{A}_{n-1}(n \geq 2), \tilde{D}_{n}(n \geq 4), \tilde{C}_{n}(n \geq$ 2), $\tilde{B}_{n}(n \geq 3), \tilde{E}_{n}(n=6,7,8), \tilde{F}_{4}, \tilde{G}_{2}$ (see 1.12-1.14) coincides with the collection of infinite, tame, irreducible Coxeter groups (or affine Weyl groups).

1.16. Let $W, S$ be an affine Weyl group. Let $\mathcal{T}$ be the union of all finite conjugacy classes in $W$. Then $\mathcal{T}$ is a normal, finitely generated free abelian subgroup of $W$ of finite index. Let $S_{\text {min }}$ be the set of all $s \in S$ such that the obvious composition $\langle S-\{s\}\rangle \rightarrow W \rightarrow W / \mathcal{T}$ is an isomorphism. (This composition is injective for any $s \in S$.) Now $S_{\text {min }} \neq \emptyset$. We describe $S_{\text {min }}$ in each case.

If $W$ is of type $\tilde{A}_{n-1}$ we have $S_{\min }=S$. In the setup of $1.13, S_{\min }$ corresponds to the following vertices of the Coxeter graph: $1, p$, if $W$ is of type $\tilde{C}_{p} ; 1, \tilde{1}$, if $W$ is of type $\tilde{B}_{p} ; 1, \tilde{1},(p-1), \widetilde{(p-1)}$, if $W$ is of type $\tilde{D}_{p}$. In the setup of 1.14 , if $W$ is of type $\tilde{E}_{n}$ then $S_{\min }$ corresponds to the following vertices of the Coxeter graph: $(1,0,0),(0,1,0),(0,0,1)$ if $W$ is of type $\tilde{E}_{6} ;(1,0,0),(0,1,0)$ if $W$ is of type $\tilde{E}_{7}$; $(1,0,0)$ if $W$ is of type $\tilde{E}_{8}$. If $W$ is of type $\tilde{F}_{4}$ then $S_{\min }=\left\{s_{1,0,0}\right\}$; if $W$ is of type $\tilde{G}_{2}$ then $S_{\min }=\left\{s_{1}\right\}$.

1.17. For a Coxeter group $W, S$ we denote by $A_{W}$ the group of all automorphisms of $W$ that map $S$ into itself. (This is also the group of automorphisms of the corresponding Coxeter graph.)

1.18. Let $W, S$ be an affine Weyl group. Let $\mathcal{T} \subset W$ be as in 1.16 . Let $\Omega$ be the set of all $a \in A_{W}$ such that there exists $w \in W$ with $a(t)=w t w^{-1}$ for all $t \in \mathcal{T}$. Now $\Omega$ is a commutative normal subgroup of $A_{W}$. The action of $A_{W}$ on $W$ restricts to an action of $\Omega$ on $S_{\text {min }}$ which is simply transitive.

1.19. For any $I \subset S$, let $W_{I}=\langle I\rangle$. Then $\left(W_{I}, I\right)$ is a Coxeter group whose Coxeter matrix is a submatrix of that of $W, S$. See $\S 9$.

1.20. Let $W, S$ be an affine Weyl group. Let $s \in S_{\min }$. Then $W_{S-\{s\}}, S-\{s\}$ is a finite Coxeter group.

A finite Coxeter group is said to be of type $A_{n-1}(n \geq 2)$ (resp. $C_{n}(n \geq 2)$, $\left.B_{n}(n \geq 3), D_{n}(n \geq 4), E_{n}(n=6,7,8), F_{4}, G_{2}\right)$ if it is isomorphic to $W_{S-\{s\}}$ for some $W, S, s$ as above, where $W$ has type $\tilde{A}_{n-1}(n \geq 2)$ (resp. $\tilde{C}_{n}(n \geq 2)$, $\left.\tilde{B}_{n}(n \geq 3), \tilde{D}_{n}(n \geq 4), \tilde{E}_{n}(n=6,7,8), \tilde{F}_{4}, \tilde{G}_{2}\right)$.

The collection of Coxeter groups of type $A_{n-1}(n \geq 2), C_{n}(n \geq 2), B_{n}(n \geq 3)$, $D_{n}(n \geq 4), E_{n}(n=6,7,8), F_{4}, G_{2}$ coincides with the collection of finite, integral, irreducible Coxeter groups $\neq\{1\}$ (or Weyl groups). The group $W=\{1\}$ with $S=\emptyset$ is also considered to be a Weyl group. Note that the types $C_{n}$ and $B_{n}$ coincide for $n \geq 3$.

1.21. Let $W, S$ be a Weyl group of type $E_{8}$. Let $W^{\prime}$ be the subgroup of $W$ generated by

$$
s_{2,0,0} s_{0,2,0}, s_{3,0,0} s_{0,4,0}, s_{4,0,0} s_{6,0,0}, s_{5,0,0} s_{0,0,3}
$$


(The index of a generator of $W$ is the corresponding vertex of the graph, see 1.13.) Then $W^{\prime}$ is a (finite, non-integral) Coxeter group on these generators, said to be of type $H_{4}$. This description of $H_{4}$ appeared in [L4].

\section{Partial order ON $W$}

2.1. Let $W, S$ be a Coxeter group. Let $y, w$ be two elements of $W$. Following Chevalley, we say that $y \leq w$ if there exists a sequence $y=y_{0}, y_{1}, y_{2}, \ldots, y_{n}=w$ in $W$ such that $l\left(y_{k}\right)-l\left(y_{k-1}\right)=1$ for $k \in[1, n]$ and $y_{k} y_{k-1}^{-1} \in T$ (or equivalently $y_{k-1} y_{k}^{-1} \in T$, or $y_{k}^{-1} y_{k-1} \in T$, or $\left.y_{k-1}^{-1} y_{k} \in T\right)$ for $k \in[1, n]$. This is a partial order on $W$. Note that $y \leq w$ implies $y^{-1} \leq w^{-1}$ and $l(y) \leq l(w)$. We write $y<w$ or $w>y$ instead of $y \leq w, y \neq w$. If $w \in W, s \in S$ then,

$s w<w$ if and only if $l(s w)=l(w)-1$;

$s w>w$ if and only if $l(s w)=l(w)+1$.

Lemma 2.2. Let $w=s_{1} s_{2} \ldots s_{q}$ be a reduced expression in $W$ and let $t \in T$. The following are equivalent:

(i) $U\left(w^{-1}\right)(\epsilon, t)=\left(-\epsilon, w^{-1} t w\right)$ for $\epsilon= \pm 1$;

(ii) $t=s_{1} s_{2} \ldots s_{i} \ldots s_{2} s_{1}$ for some $i \in[1, q]$;

(iii) $l(t w)<l(w)$.

The equivalence of (i),(ii) has been proved in 1.6.

Proof of (ii) $\Longrightarrow$ (iii). Assume that (ii) holds. Then $t w=s_{1} \ldots s_{i-1} s_{i+1} \ldots s_{q}$ hence $l(t w)<q$ and (iii) holds.

Proof of (iii) $\Longrightarrow$ (i). First we check that

$$
U(t)(\epsilon, t)=(-\epsilon, t)
$$

If $t \in S,(\mathrm{a})$ is clear. If (a) is true for $t$ then it is also true for sts where $s \in S$. Indeed,

$$
\begin{aligned}
U(s t s)(\epsilon, s t s) & =U_{s} U(t) U_{s}(\epsilon, s t s)=U_{s} U(t)\left(\epsilon(-1)^{\delta_{s, s t s}}, t\right)=U_{s}\left(-\epsilon(-1)^{\delta_{s, s t s}}, t\right) \\
& =\left(-\epsilon(-1)^{\delta_{s, s t s}+\delta_{s, t}}, t\right)=(-\epsilon, t)
\end{aligned}
$$

(a) follows. Assume now that (i) does not hold; thus, $U\left(w^{-1}\right)(\epsilon, t)=\left(\epsilon, w^{-1} t w\right)$. Then

$$
\begin{aligned}
U\left((t w)^{-1}\right)(\epsilon, t) & =U\left(w^{-1}\right) U(t)(\epsilon, t)=U\left(w^{-1}\right)(-\epsilon, t)=\left(-\epsilon, w^{-1} t w\right) \\
& =\left(-\epsilon,(t w)^{-1} t(t w)\right)
\end{aligned}
$$

Since $(\mathrm{i}) \Longrightarrow$ (iii) we deduce that $l(w)<l(t w)$; thus, (iii) does not hold. The lemma is proved. 
Lemma 2.3. Let $y, z \in W$ and let $s \in S$. If $s y \leq z<s z$, then $y \leq s z$.

We argue by induction on $l(z)-l(s y)$. If $l(z)-l(s y)=0$ then $z=s y$ and the result is clear. Now assume that $l(z)>l(s y)$. Then $s y<z$. We can assume that $s y<y$ (otherwise the result is trivial). We can find $t \in T$ such that $s y<t s y \leq z$ and $l(t s y)=l(s y)+1$. If $t=s$, then $y \leq z$ and we are done. Hence we may assume that $t \neq s$. We show that

$$
y<s t s y
$$

Assume that (a) does not hold. Then $y, t s y$, sy, stsy have lengths $q+1, q+1, q, q$. We can find a reduced expression $y=s s_{1} s_{2} \ldots s_{q}$. Since $l(s t s y)<l(y)$, we see from 2.2 that either $s t s=s s_{1} \ldots s_{i} \ldots s_{1} s$ for some $i \in[1, q]$ or $s t s=s$. (This last case has been excluded.) It follows that

$$
t s y=s_{1} \ldots s_{i} \ldots s_{1} s s s_{1} s_{2} \ldots s_{q}=s_{1} \ldots s_{i-1} s_{i+1} \ldots s_{q} .
$$

Thus, $l(t s y) \leq q-1$, a contradiction. Thus, (a) holds. Let $y^{\prime}=$ stsy. We have $s y^{\prime} \leq z \leq s z$ and $l(z)-l\left(s y^{\prime}\right)<l(z)-l(s y)$. By the induction hypothesis, $y^{\prime} \leq s z$. We have $y<y^{\prime}$ by (a), hence $y \leq s z$. The lemma is proved.

Proposition 2.4. The following three conditions on $y, w \in W$ are equivalent:

(i) $y \leq w$;

(ii) for any reduced expression $w=s_{1} s_{2} \ldots s_{q}$ there exists a subsequence $i_{1}<$ $i_{2}<\cdots<i_{r}$ of $1,2, \ldots, q$ such that $y=s_{i_{1}} s_{i_{2}} \ldots s_{i_{r}}, r=l(y)$;

(iii) there exists a reduced expression $w=s_{1} s_{2} \ldots s_{q}$ and a subsequence $i_{1}<$ $i_{2}<\cdots<i_{r}$ of $1,2, \ldots, q$ such that $y=s_{i_{1}} s_{i_{2}} \ldots s_{i_{r}}$.

Proof of $(\mathrm{i}) \Longrightarrow$ (ii). We may assume that $y<w$. Let $y=y_{0}, y_{1}, y_{2}, \ldots, y_{n}=w$ be as in 2.1. Let $w=s_{1} s_{2} \ldots s_{q}$ be a reduced expression. Since $y_{n-1} y_{n}^{-1} \in$ $T, l\left(y_{n-1}\right)=l\left(y_{n}\right)-1$, we see from 2.2 that there exists $i \in[1, q]$ such that $y_{n-1} y_{n}^{-1}=s_{1} s_{2} \ldots s_{i} \ldots s_{2} s_{1}$ hence $y_{n-1}=s_{1} s_{2} \ldots s_{i-1} s_{i+1} \ldots s_{q}$ (a reduced expression). Similarly, since $y_{n-2} y_{n-1}^{-1} \in T, l\left(y_{n-2}\right)=l\left(y_{n-1}\right)-1$, we see from 2.2 (applied to $y_{n-1}$ ) that there exists $j \in[1, q]-\{i\}$ such that $y_{n-2}$ equals

$$
s_{1} s_{2} \ldots s_{i-1} s_{i+1} \ldots s_{j-1} s_{j+1} \ldots s_{q} \text { or } s_{1} s_{2} \ldots s_{j-1} s_{j+1} \ldots s_{i-1} s_{i+1} \ldots s_{q}
$$

(depending on whether $i<j$ or $i>j$ ). Continuing in this way we see that $y$ is of the required form.

The proof of (ii) $\Longrightarrow$ (iii) is trivial.

Proof of (iii) $\Longrightarrow(\mathrm{i})$. Assume that $w=s_{1} s_{2} \ldots s_{q}$ (reduced expression) and $y=s_{i_{1}} s_{i_{2}} \ldots s_{i_{r}}$ where $i_{1}<i_{2}<\cdots<i_{r}$ is a subsequence of $1,2, \ldots, q$. We argue by induction on $q$. If $q=0$ there is nothing to prove. Now assume $q>0$.

If $i_{1}>1$, then the induction hypothesis is applicable to $y, w^{\prime}=s_{2} \ldots s_{q}$ and yields $y \leq w^{\prime}$. But $w^{\prime} \leq w$ hence $y \leq w$. If $i_{1}=1$ then the induction hypothesis is applicable to $y^{\prime}=s_{i_{2}} \ldots s_{i_{r}}, w^{\prime}=s_{2} \ldots s_{q}$ and yields $y^{\prime} \leq w^{\prime}$. Thus, $s_{1} y \leq s_{1} w<$ $w$. By 2.3 we then have $y \leq w$. The proposition is proved. 
Corollary 2.5. Let $y, z \in W$ and let $s \in S$.

(a) Assume that $s z<z$. Then $y \leq z \Leftrightarrow s y \leq z$.

(b) Assume that $y<s y$. Then $y \leq z \Leftrightarrow y \leq s z$.

We prove (a). We can find a reduced expression of $z$ of form $z=s s_{1} s_{2} \ldots s_{q}$. Assume that $y \leq z$. By 2.4 we can find a subsequence $i_{1}<i_{2}<\cdots<i_{r}$ of $1,2, \ldots, q$ such that either $y=s_{i_{1}} s_{i_{2}} \ldots s_{i_{r}}$ or $y=s s_{i_{1}} s_{i_{2}} \ldots s_{i_{r}}$. In the first case we have $s y=s s_{i_{1}} s_{i_{2}} \ldots s_{i_{r}}$ and in the second case we have $s y=s_{i_{1}} s_{i_{2}} \ldots s_{i_{r}}$. In both cases, $s y \leq z$ by 2.4. The same argument shows that, if $s y \leq z$ then $y \leq z$. This proves (a).

We prove (b). Assume that $y \leq z$. We must prove that $y \leq s z$. If $z<s z$, this is clear. Thus we may assume that $s z<z$. We can find a reduced expression of $z$ of form $z=s s_{1} s_{2} \ldots s_{q}$. By 2.4 we can find a subsequence $i_{1}<i_{2}<\cdots<i_{r}$ of $1,2, \ldots, q$ such that either

$$
y=s_{i_{1}} s_{i_{2}} \ldots s_{i_{r}}, l(y)=r \text { or } y=s s_{i_{1}} s_{i_{2}} \ldots s_{i_{r}}, l(y)=r+1 .
$$

In the second case we have $l(s y)=r<l(y)$, contradicting $y<s y$. Thus we are in the first case. Hence $y$ is the product of a subsequence of $s_{1}, s_{2}, \ldots, s_{q}$ and using again 2.4, we deduce that $y \leq s z$ (note that $s z=s_{1} s_{2} \ldots s_{q}$ is a reduced expression). The lemma is proved.

\section{THE ALGEBRA $\mathcal{H}$}

3.1. Let $W, S$ be a Coxeter group. A map $L: W \rightarrow \mathbf{Z}$ is said to be a weight function for $W$ if $L\left(w w^{\prime}\right)=L(w)+L\left(w^{\prime}\right)$ for any $w, w^{\prime} \in W$ such that $l\left(w w^{\prime}\right)=$ $l(w)+l\left(w^{\prime}\right)$. We will assume that a weight function $L: W \rightarrow \mathbf{Z}$ is fixed; we then say that $W, L$ is a weighted Coxeter group. (For example we could take $L=l$; in that case we say that we are in the split case.) Note that $L$ is determined by its values on $S$ which are subject only to the condition

$L(s)=L\left(s^{\prime}\right)$ for any $s \neq s^{\prime}$ in $S$ such that $m_{s, s^{\prime}}$ is finite and odd.

We have $L(w)=L\left(w^{-1}\right)$ for all $w \in W$.

Let $\mathcal{A}=\mathbf{Z}\left[v, v^{-1}\right]$ where $v$ is an indeterminate. For $s \in S$ we set $v_{s}=v^{L(s)} \in \mathcal{A}$.

3.2. Let $\mathcal{H}$ be the $\mathcal{A}$-algebra defined by the generators $T_{s}(s \in S)$ and the relations

$$
\left(T_{s}-v_{s}\right)\left(T_{s}+v_{s}^{-1}\right)=0 \text { for } s \in S
$$

$$
T_{s} T_{s^{\prime}} T_{s} \cdots=T_{s^{\prime}} T_{s} T_{s^{\prime}} \ldots
$$

(both products have $m_{s, s^{\prime}}$ factors) for any $s \neq s^{\prime}$ in $S$ such that $m_{s, s^{\prime}}<\infty ; \mathcal{H}$ is called the Iwahori-Hecke algebra. We have $T_{1}=1$, the unit element of $\mathcal{H}$.

For $w \in W$ we define $T_{w} \in \mathcal{H}$ by $T_{w}=T_{s_{1}} T_{s_{2}} \ldots T_{s_{q}}$, where $w=s_{1} s_{2} \ldots s_{q}$ is a reduced expression in $W$. By (b) and 1.9, $T_{w}$ is independent of the choice of reduced expression. From the definitions it is clear that for $s \in S, w \in W$ we have

$$
T_{s} T_{w}=T_{s w} \text { if } l(s w)=l(w)+1,
$$




$$
T_{s} T_{w}=T_{s w}+\left(v_{s}-v_{s}^{-1}\right) T_{w} \text { if } l(s w)=l(w)-1 .
$$

In particular, the $\mathcal{A}$-submodule of $\mathcal{H}$ generated by $\left\{T_{w} ; w \in W\right\}$ is a left ideal of $\mathcal{H}$. It contains $1=T_{1}$ hence it is the whole of $\mathcal{H}$. Thus $\left\{T_{w} ; w \in W\right\}$ generates the $\mathcal{A}$-module $\mathcal{H}$.

Proposition 3.3. $\left\{T_{w} ; w \in W\right\}$ is an $\mathcal{A}$-basis of $\mathcal{H}$.

We follow the lines of the proof in [Bo, Ex.23, p.55].

We consider the free $\mathcal{A}$-module $\mathcal{E}$ with basis $\left(e_{w}\right)_{w \in W}$. For any $s \in S$ we define $\mathcal{A}$-linear maps $P_{s}, Q_{s}: \mathcal{E} \rightarrow \mathcal{E}$ by

$P_{s}\left(e_{w}\right)=e_{s w}$ if $l(s w)=l(w)+1$,

$P_{s}\left(e_{w}\right)=e_{s w}+\left(v_{s}-v_{s}^{-1}\right) e_{w}$ if $l(s w)=l(w)-1$;

$Q_{s}\left(e_{w}\right)=e_{w s}$ if $l(w s)=l(w)+1$,

$Q_{s}\left(e_{w}\right)=e_{w s}+\left(v_{s}-v_{s}^{-1}\right) e_{w}$ if $l(w s)=l(w)-1$.

We shall continue the proof assuming that

(a) $P_{s} Q_{t}=Q_{t} P_{s}$ for any $s, t$ in $S$.

Let $\mathfrak{A}$ be the $\mathcal{A}$-subalgebra with 1 of $\operatorname{End}(\mathcal{E})$ generated by $\left\{P_{s} ; s \in S\right\}$. The map $\mathfrak{A} \rightarrow \mathcal{E}$ given by $\pi \mapsto \pi\left(e_{1}\right)$ is surjective. Indeed, if $w=s_{1} s_{2} \ldots s_{q}$ is a reduced expression in $W$, then $e_{w}=P_{s_{1}} \ldots P_{s_{q}} e_{1}$. Assume now that $\pi \in \mathfrak{A}$ satisfies $\pi\left(e_{1}\right)=0$. Let $\pi^{\prime}=Q_{s_{q}} \ldots Q_{s_{1}}$. By (a) we have $\pi \pi^{\prime}=\pi^{\prime} \pi$ hence

$$
0=\pi^{\prime} \pi\left(e_{1}\right)=\pi \pi^{\prime}\left(e_{1}\right)=\pi\left(Q_{s_{q}} \ldots Q_{s_{1}}\left(e_{1}\right)\right)=\pi\left(e_{w}\right) .
$$

Since $w$ is arbitrary, it follows that $\pi=0$. We see that the map $\mathfrak{A} \rightarrow \mathcal{E}$ is injective, hence an isomorphism of $\mathcal{A}$-modules. Using this isomorphism we transport the algebra structure of $\mathfrak{A}$ to an algebra structure on $\mathcal{E}$ with unit element $e_{1}$. For this algebra structure we have $P_{s}\left(e_{1}\right) \pi\left(e_{1}\right)=P_{s}\left(\pi\left(e_{1}\right)\right)$ for $s \in S, \pi \in \mathfrak{A}$. Hence $e_{s} e_{w}=P_{s}\left(e_{w}\right)$ for any $w \in W, s \in S$. It follows that

(b) $e_{s} e_{w}=e_{s w}$ if $l(s w)=l(w)+1$,

(c) $e_{s} e_{w}=e_{s w}+\left(v_{s}-v_{s}^{-1}\right) e_{w}$ if $l(s w)=l(w)-1$.

From (b) it follows that, if $w=s_{1} s_{2} \ldots s_{q}$ is a reduced expression, then $e_{w}=$ $e_{s_{1}} e_{s_{2}} \ldots e_{s_{q}}$. In particular, if $s \neq s^{\prime}$ in $S$ are such that $m=m_{s, s^{\prime}}<\infty$ then $e_{s} e_{s^{\prime}} e_{s} \cdots=e_{s^{\prime}} e_{s} e_{s^{\prime}} \ldots$ (both products have $m$ factors); indeed, this follows from the equality $e_{s s^{\prime} s \ldots}=e_{s^{\prime} s s^{\prime} \ldots}$ (see 1.4). From (c) we deduce that $e_{s}^{2}=1+\left(v_{s}-\right.$ $\left.v_{s}^{-1}\right) e_{s}$ for $s \in S$, or that $\left(e_{s}-v_{s}\right)\left(e_{s}+v_{s}^{-1}\right)=0$. We see that there is a unique algebra homomorphism $\mathcal{H} \rightarrow \mathcal{E}$ preserving 1 such that $T_{s} \mapsto e_{s}$ for all $s \in S$. It takes $T_{w}$ to $e_{w}$ for any $w \in W$. Assume now that $a_{w} \in \mathcal{A}(w \in W)$ are zero for all but finitely many $w$ and that $\sum_{w} a_{w} T_{w}=0$ in $\mathcal{H}$. Applying $\mathcal{H} \rightarrow \mathcal{E}$ we obtain $\sum_{w} a_{w} e_{w}=0$. Since $\left(e_{w}\right)$ is a basis of $\mathcal{E}$, it follows that $a_{w}=0$ for all $w$. Thus, $\left\{T_{w} ; w \in W\right\}$ is an $\mathcal{A}$-basis of $\mathcal{H}$. This completes the proof, modulo the verification of (a).

We prove (a). Let $w \in W$. We distinguish six cases.

Case 1. swt, sw, wt, $w$ have lengths $q+2, q+1, q+1, q$. Then

$$
P_{s} Q_{t}\left(e_{w}\right)=Q_{t} P_{s}\left(e_{w}\right)=e_{s w t} .
$$


Case 2. $w, s w, w t, s w t$ have lengths $q+2, q+1, q+1, q$. Then

$$
\begin{aligned}
& P_{s} Q_{t}\left(e_{w}\right)=Q_{t} P_{s}\left(e_{w}\right) \\
& =e_{s w t}+\left(v_{t}-v_{t}^{-1}\right) e_{s w}+\left(v_{s}-v_{s}^{-1}\right) e_{w t}+\left(v_{t}-v_{t}^{-1}\right)\left(v_{s}-v_{s}^{-1}\right) e_{w} .
\end{aligned}
$$

Case 3. wt, swt, $w, s w$ have lengths $q+2, q+1, q+1, q$. Then

$$
P_{s} Q_{t}\left(e_{w}\right)=Q_{t} P_{s}\left(e_{w}\right)=e_{s w t}+\left(v_{s}-v_{s}^{-1}\right) e_{w t} .
$$

Case 4. sw, swt, $w, w t$ have lengths $q+2, q+1, q+1, q$. Then

$$
P_{s} Q_{t}\left(e_{w}\right)=Q_{t} P_{s}\left(e_{w}\right)=e_{s w t}+\left(v_{t}-v_{t}^{-1}\right) e_{s w} .
$$

Case 5. swt, $w, w t, s w$ have lengths $q+1, q+1, q, q$. Then

$$
\begin{aligned}
& P_{s} Q_{t}\left(e_{w}\right)=e_{s w t}+\left(v_{t}-v_{t}^{-1}\right) e_{s w}+\left(v_{t}-v_{t}^{-1}\right)\left(v_{s}-v_{s}^{-1}\right) e_{w} \\
& Q_{t} P_{s}\left(e_{w}\right)=e_{s w t}+\left(v_{s}-v_{s}^{-1}\right) e_{w t}+\left(v_{t}-v_{t}^{-1}\right)\left(v_{s}-v_{s}^{-1}\right) e_{w} .
\end{aligned}
$$

Case 6. sw, wt, w, swt have lengths $q+1, q+1, q, q$. Then

$$
\begin{aligned}
& P_{s} Q_{t}\left(e_{w}\right)=e_{s w t}+\left(v_{s}-v_{s}^{-1}\right) e_{w t} \\
& Q_{t} P_{s}\left(e_{w}\right)=e_{s w t}+\left(v_{t}-v_{t}^{-1}\right) e_{s w} .
\end{aligned}
$$

In case 5 we have $L(t)+L(w t)=L(w)=L(s w t)=L(s)+L(w t)$ hence $L(t)=L(s)$ and $v_{s}=v_{t}$. In case 6 we have $L(t)+L(s w t)=L(s w)=L(w t)=L(s)+L(s w t)$, hence $L(t)=L(s)$ and $v_{s}=v_{t}$. In cases 5,6 we have $s w=w t$ by 1.10 . Hence $P_{s} Q_{t}\left(e_{w}\right)=Q_{t} P_{s}\left(e_{w}\right)$ in each case. The proposition is proved.

3.4. There is a unique involutive antiautomorphism $h \mapsto h^{b}$ of the algebra $\mathcal{H}$ which carries $T_{s}$ to $T_{s}$ for any $s \in S$. It carries $T_{w}$ to $T_{w^{-1}}$ for any $w \in W$.

3.5. There is a unique algebra involution of $\mathcal{H}$ denoted $h \mapsto h^{\dagger}$ such that $T_{s}^{\dagger}=$ $-T_{s}^{-1}$ for any $s \in S$. We have $T_{w}^{\dagger}=\operatorname{sgn}(w) T_{w^{-1}}^{-1}$ for any $w \in W$.

\section{THE BAR OPERATOR}

4.1. We preserve the setup of 3.1. For $s \in S$, the element $T_{s} \in \mathcal{H}$ is invertible: $T_{s}^{-1}=T_{s}-\left(v_{s}-v_{s}^{-1}\right)$. It follows that $T_{w}$ is invertible for each $w \in W$; if $w=s_{1} s_{2} \ldots s_{q}$ is a reduced expression in $W$, then $T_{w}^{-1}=T_{s_{q}}^{-1} \ldots T_{s_{2}}^{-1} T_{s_{1}}^{-1}$.

Let $^{-}: \mathcal{A} \rightarrow \mathcal{A}$ be the ring involution which takes $v^{n}$ to $v^{-n}$ for any $n \in \mathbf{Z}$. 
Lemma 4.2. (a) There is a unique ring homomorphism ${ }^{-}: \mathcal{H} \rightarrow \mathcal{H}$ which is $\mathcal{A}$-semilinear with respect to ${ }^{-}: \mathcal{A} \rightarrow \mathcal{A}$ and satisfies $\bar{T}_{s}=T_{s}^{-1}$ for any $s \in S$.

(b) This homomorphism is involutive. It takes $T_{w}$ to $T_{w^{-1}}^{-1}$ for any $w \in W$.

The following identities can be deduced easily from $3.2(\mathrm{a}),(\mathrm{b}),(\mathrm{d})$ :

$\left(T_{s}^{-1}-v_{s}^{-1}\right)\left(T_{s}^{-1}+v_{s}\right)=0$ for $s \in S$, $T_{s}^{-1} T_{s^{\prime}}^{-1} T_{s}^{-1} \cdots=T_{s^{\prime}}^{-1} T_{s}^{-1} T_{s^{\prime}}^{-1} \ldots$

(both products have $m_{s, s^{\prime}}$ factors) for any $s \neq s^{\prime}$ in $S$ such that $m_{s, s^{\prime}}<\infty$; (a) follows.

We prove (b). Let $s \in S$. Applying ${ }^{-}$to $T_{s} \bar{T}_{s}=1$ gives $\bar{T}_{s} \overline{\bar{T}}_{s}=1$. We have also $\bar{T}_{s} T_{s}=1$ hence $\overline{\bar{T}}_{s}=T_{s}$. It follows that the square of ${ }^{-}$is 1 . The second assertion of (b) is immediate. The lemma is proved.

4.3. For any $w \in W$ we can write uniquely $\bar{T}_{w}=\sum_{y \in W} \bar{r}_{y, w} T_{y}$ where $r_{y, w} \in \mathcal{A}$ are zero for all but finitely many $y$.

Lemma 4.4. Let $w \in W$ and $s \in S$ be such that $w>$ sw. For $y \in W$ we have

$r_{y, w}=r_{s y, s w}$ if $s y<y$,

$r_{y, w}=r_{s y, s w}+\left(v_{s}-v_{s}^{-1}\right) r_{y, s w}$ if $s y>y$.

We have

$$
\begin{aligned}
\bar{T}_{w} & =T_{s}^{-1} \bar{T}_{s w}=\left(T_{s}-\left(v_{s}-v_{s}^{-1}\right)\right) \sum_{y} \bar{r}_{y, s w} T_{y} \\
& =\sum_{y} \bar{r}_{y, s w} T_{s y}-\sum_{y}\left(v_{s}-v_{s}^{-1}\right) \bar{r}_{y, s w} T_{y}+\sum_{y ; s y<y}\left(v_{s}-v_{s}^{-1}\right) \bar{r}_{y, s w} T_{y} \\
& =\sum_{y} \bar{r}_{s y, s w} T_{y}-\sum_{y ; s y>y}\left(v_{s}-v_{s}^{-1}\right) \bar{r}_{y, s w} T_{y} .
\end{aligned}
$$

The lemma follows.

Lemma 4.5. For any $y, w$ we have $\bar{r}_{y, w}=\operatorname{sgn}(y w) r_{y, w}$.

We argue by induction on $l(w)$. If $l(w)=0$, then $w=1$ and the result is obvious. Assume now that $l(w) \geq 1$. We can find $s \in S$ such that $w>s w$. Assume first that $s y<y$. From 4.4 we see, using the induction hypothesis, that

$$
\bar{r}_{y, w}=\bar{r}_{s y, s w}=\operatorname{sgn}(s y s w) r_{s y, s w}=\operatorname{sgn}(y w) r_{y, w}
$$

Assume next that $s y>y$. From 4.4 we see, using the induction hypothesis, that

$$
\begin{aligned}
\bar{r}_{y, w} & =\bar{r}_{s y, s w}+\left(v_{s}^{-1}-v_{s}\right) \bar{r}_{y, s w}=\operatorname{sgn}(s y s w) r_{s y, s w}+\left(v_{s}^{-1}-v_{s}\right) \operatorname{sgn}(y s w) r_{y, s w} \\
& =\operatorname{sgn}(y w)\left(r_{s y, s w}+\left(v_{s}-v_{s}^{-1}\right) r_{y, s w}\right)=\operatorname{sgn}(y w) r_{y, w} .
\end{aligned}
$$

The lemma is proved. 
Lemma 4.6. For any $x, z \in W$ we have $\sum_{y} \bar{r}_{x, y} r_{y, z}=\delta_{x, z}$.

Using the fact that ${ }^{-}$is an involution, we have

$$
T_{z}=\overline{\bar{T}}_{z}=\overline{\sum_{y} \bar{r}_{y, z} T_{y}}=\sum_{y} r_{y, z} \bar{T}_{y}=\sum_{y} \sum_{x} r_{y, z} \bar{r}_{x, y} T_{x} .
$$

We now compare the coefficients of $T_{x}$ on both sides. The lemma follows.

Proposition 4.7. Let $y, w \in W$.

(a) If $r_{y, w} \neq 0$, then $y \leq w$.

(b) Assume that $L(s)>0$ for all $s \in S$. If $y \leq w$, then

$r_{y, w}=v^{L(w)-L(y)} \bmod v^{L(w)-L(y)-1} \mathbf{Z}\left[v^{-1}\right]$,

$r_{y, w}=\operatorname{sgn}(y w) v^{-L(w)+L(y)} \bmod v^{-L(w)+L(y)+1} \mathbf{Z}[v]$.

(c) Without assumption on $L, r_{y, w} \in v^{L(w)-L(y)} \mathbf{Z}\left[v^{2}, v^{-2}\right]$.

We prove (a) by induction on $l(w)$. If $l(w)=0$ then $w=1$ and the result is obvious. Assume now that $l(w) \geq 1$. We can find $s \in S$ such that $w>s w$. Assume first that $s y<y$. From 4.4 we see that $r_{s y, s w} \neq 0$ hence, by the induction hypothesis, $s y \leq s w$. Thus $s y \leq s w<w$ and, by 2.3 , we deduce $y \leq w$. Assume next that $s y>y$. From 4.4 we see that either $r_{s y, s w} \neq 0$ or $r_{y, s w} \neq 0$ hence, by the induction hypothesis, $s y \leq s w$ or $y \leq s w$. Combining this with $y<s y$ and $s w<w$ we see that $y \leq w$. This proves (a).

We prove the first assertion of (b) by induction on $l(w)$. If $l(w)=0$ then $w=1$ and the result is obvious. Assume now that $l(w) \geq 1$. We can find $s \in S$ such that $w>s w$. Assume first that $s y<y$. Then we have also $s y<w$ and, using 2.5(b), we deduce $s y \leq s w$. By the induction hypothesis,

$$
\begin{aligned}
r_{s y, s w} & =v^{L(s w)-L(s y)}+\text { strictly lower powers } \\
& =v^{L(w)-L(y)}+\text { strictly lower powers. }
\end{aligned}
$$

But $r_{y, w}=r_{s y, s w}$ and the result follows. Assume next that $s y>y$. From $y<$ $s y, y \leq w$ we deduce using 2.5(b) that $y \leq s w$. By the induction hypothesis, we have $r_{y, s w}=v^{L(s w)-L(y)}+$ strictly lower powers. Hence

$$
\begin{aligned}
& \left(v_{s}-v_{s}^{-1}\right) r_{y, s w}=v^{L(s)} v^{L(s w)-L(y)}+\text { strictly lower powers } \\
& =v^{L(w)-L(y)}+\text { strictly lower powers. }
\end{aligned}
$$

On the other hand, if $s y \leq s w$, then by the induction hypothesis,

$$
\begin{aligned}
& r_{s y, s w}=v^{L(s w)-L(s y)}+\text { strictly lower powers } \\
& =v^{L(w)-L(y)-2 L(s)}+\text { strictly lower powers }
\end{aligned}
$$

while if $s y \not \leq s w$ then $r_{s y, s w}=0$ by (a). Thus, in $r_{y, w}=r_{s y, s w}+\left(v_{s}-v_{s}^{-1}\right) r_{y, s w}$, the term $r_{s y, s w}$ contributes only powers of $v$ which are strictly smaller than $L(w)-L(y)$ 
hence $r_{y, w}=v^{L(w)-L(y)}+$ strictly lower powers. This proves the first assertion of (b). The second assertion of (b) follows from the first using 4.5.

We prove (c) by induction on $l(w)$. If $l(w)=0$ then $w=1$ and the result is obvious. Assume now that $l(w) \geq 1$. We can find $s \in S$ such that $w>s w$. Assume first that $s y<y$. By the induction hypothesis,

$$
r_{y, w}=r_{s y, s w} \in v^{L(s w)-L(s y)} \mathbf{Z}\left[v^{2}, v^{-2}\right]=v^{L(w)-L(y)} \mathbf{Z}\left[v^{2}, v^{-2}\right]
$$

as required. Assume next that $s y>y$. By the induction hypothesis,

$$
\begin{aligned}
& r_{y, w}=r_{s y, s w}+\left(v_{s}-v_{s}^{-1}\right) r_{y, s w} \\
& \in v^{L(s w)-L(s y)} \mathbf{Z}\left[v^{2}, v^{-2}\right]+v^{L(s)} v^{L(s w)-L(y)} \mathbf{Z}\left[v^{2}, v^{-2}\right]=v^{L(w)-L(y)} \mathbf{Z}\left[v^{2}, v^{-2}\right],
\end{aligned}
$$

as required. The proposition is proved.

Proposition 4.8. For $x<z$ in $W$ we have $\sum_{y ; x \leq y \leq z} \operatorname{sgn}(y)=0$ (D.N. Verma).

Using 4.5 we can rewrite 4.6 (in our case) in the form

$$
\sum_{y} \operatorname{sgn}(x y) r_{x, y} r_{y, z}=0
$$

Here we may restrict the summation to $y$ such that $x \leq y \leq z$. In the rest of the proof we shall take $L=l$. Then 4.7(b) holds and we see that if $x \leq y \leq z$, then

$$
r_{x, y} r_{y, z}=v^{l(y)-l(x)} v^{l(z)-l(y)}+\text { strictly lower powers of } v \text {. }
$$

Hence (a) states that

$\sum_{y ; x \leq y \leq z} \operatorname{sgn}(x y) v^{l(z)-l(x)}+$ strictly lower powers of $v$ is 0.

In particular $\sum_{y ; x \leq y \leq z} \operatorname{sgn}(x y)=0$. The proposition is proved.

4.9. Now ${ }^{-}: \mathcal{H} \rightarrow \mathcal{H}$ commutes with $h \mapsto h^{b}$. Hence

(a) $r_{y^{-1}, w^{-1}}=r_{y, w}$

for any $y, w \in W$. On the other hand, it is clear that ${ }^{-}: \mathcal{H} \rightarrow \mathcal{H}$ and ${ }^{\dagger}: \mathcal{H} \rightarrow \mathcal{H}$ commute.

\section{The Elements $c_{w}$}

5.1. We preserve the setup of 3.1. For any $n \in \mathbf{Z}$ let

$$
\begin{gathered}
\mathcal{A}_{\leq n}=\oplus_{m ; m \leq n} \mathbf{Z} v^{m}, \mathcal{A}_{\geq n}=\oplus_{m ; m \geq n} \mathbf{Z} v^{m}, \\
\mathcal{A}_{<n}=\oplus_{m ; m<n} \mathbf{Z} v^{m}, \mathcal{A}_{>n}=\oplus_{m ; m>n} \mathbf{Z} v^{m}, \\
\mathcal{H}_{\leq 0}=\oplus_{w} \mathcal{A}_{\leq 0} T_{w}, \mathcal{H}_{<0}=\oplus_{w} \mathcal{A}_{<0} T_{w} .
\end{gathered}
$$

We have $\mathcal{A}_{\leq 0}=\mathbf{Z}\left[v^{-1}\right], \mathcal{H}_{<0} \subset \mathcal{H}_{\leq 0} \subset \mathcal{H}$. 
Theorem 5.2. (a) Let $w \in W$. There exists a unique element $c_{w} \in \mathcal{H}_{\leq 0}$ such that $\bar{c}_{w}=c_{w}$ and $c_{w}=T_{w} \bmod \mathcal{H}_{<0}$.

(b) $\left\{c_{w} ; w \in W\right\}$ is an $\mathcal{A}_{\leq 0}$-basis of $\mathcal{H}_{\leq 0}$ and an $\mathcal{A}$-basis of $\mathcal{H}$.

We prove the existence part of (a). We will construct, for any $x$ such that $x \leq w$, an element $u_{x} \in \mathcal{A}_{\leq 0}$ such that

(c) $u_{w}=1$

(d) $u_{x} \in \mathcal{A}_{<0}, \bar{u}_{x}-u_{x}=\sum_{y ; x<y<w} r_{x, y} u_{y}$ for any $x<w$.

We argue by induction on $l(w)-l(x)$. If $l(w)-l(x)=0$ then $x=w$ and we set $u_{x}=1$. Assume now that $l(w)-l(x)>0$ and that $u_{z}$ is already defined whenever $z \leq w, l(w)-l(z)<l(w)-l(x)$ so that (c) holds and (d) holds if $x$ is replaced by any such $z$. Then the right hand side of the equality in $(\mathrm{d})$ is defined. We denote it by $a_{x} \in \mathcal{A}$. We have

$$
\begin{aligned}
a_{x}+\bar{a}_{x} & =\sum_{y ; x<y \leq w} r_{x, y} u_{y}+\sum_{y ; x<y \leq w} \bar{r}_{x, y} \bar{u}_{y} \\
& =\sum_{y ; x<y \leq w} r_{x, y} u_{y}+\sum_{y ; x<y \leq w} \bar{r}_{x, y}\left(u_{y}+\sum_{z ; y<z \leq w} r_{y, z} u_{z}\right) \\
& =\sum_{z ; z<y \leq w} r_{z, y} u_{y}+\sum_{z ; x<z \leq w} \bar{r}_{x, z} u_{z}+\sum_{z ; x<z \leq w} \bar{r}_{x, y} r_{y, z} u_{z} \\
& =\sum_{z ; x<z \leq w} \sum_{y ; x \leq y \leq z} \bar{r}_{x, y} r_{y, z} u_{z}=\sum_{z ; x<z \leq w} \delta_{x, z} u_{z}=0 .
\end{aligned}
$$

(We have used 4.6 and the equality $r_{y, y}=1$.) Since $a_{x}+\bar{a}_{x}=0$, we have $a_{x}=$ $\sum_{n \in \mathbf{Z}} \gamma_{n} v^{n}$ (finite sum) where $\gamma_{n} \in \mathbf{Z}$ satisfy $\gamma_{n}+\gamma_{-n}=0$ for all $n$ and in particular, $\gamma_{0}=0$. Then $u_{x}=\sum_{n<0} \gamma_{n} v^{n} \in \mathcal{A}_{<0}$ satisfies $\bar{u}_{x}-u_{x}=a_{x}$. This completes the inductive construction of the elements $u_{x}$. We set $c_{w}=\sum_{y ; y \leq w} u_{y} T_{y} \in \mathcal{H}_{\leq 0}$. It is clear that $c_{w}=T_{w} \bmod \mathcal{H}_{<0}$. We have

$$
\begin{aligned}
& \bar{c}_{w}=\sum_{y ; y \leq w} \bar{u}_{y} \bar{T}_{y}=\sum_{y ; y \leq w} \bar{u}_{y} \sum_{x ; x \leq y} \bar{r}_{x, y} T_{x}=\sum_{x ; x \leq w}\left(\sum_{y ; x \leq y \leq w} \bar{r}_{x, y} \bar{u}_{y}\right) T_{x} \\
& =\sum_{x ; x \leq w} u_{x} T_{x}=c_{w} .
\end{aligned}
$$

(We have used the fact that $r_{x, y} \neq 0$ implies $x \leq y$, see 4.7 , and (d).) The existence of the element $c_{w}$ is established.

To prove uniqueness, it suffices to verify the following statement:

(e) If $h \in \mathcal{H}_{<0}$ satisfies $\bar{h}=h$ then $h=0$.

We can write uniquely $h=\sum_{y \in W} f_{y} T_{y}$ where $f_{y} \in \mathcal{A}_{<0}$ are zero for all but finitely many $y$. Assume that not all $f_{y}$ are 0 . Then we can find $l_{0} \in \mathbf{N}$ such that

$$
Y_{0}:=\left\{y \in W ; f_{y} \neq 0, l(y)=l_{0}\right\} \neq \emptyset \text { and }\left\{y \in W ; f_{y} \neq 0, l(y)>l_{0}\right\}=\emptyset
$$


Now $\sum_{y} f_{y} T_{y}=\overline{\sum_{y} f_{y} T_{y}}$ implies

$$
\sum_{y \in Y_{0}} f_{y} T_{y}=\sum_{y \in Y_{0}} \overline{f_{y}} T_{y} \quad \bmod \sum_{y ; l(y)<l\left(y_{0}\right)} \mathcal{A} T_{y}
$$

hence $\overline{f_{y}}=f_{y}$ for any $y \in Y_{0}$. Since $f_{y} \in \mathcal{A}_{<0}$, it follows that $f_{y}=0$ for any $y \in Y_{0}$, a contradiction. We have proved that $f_{y}=0$ for all $y$; (e) is verified and (a) is proved.

The elements $c_{w}$ constructed in (a) (for various $w$ ) are related to the basis $T_{w}$ by a triangular matrix (with respect to $\leq$ ) with 1 on the diagonal. Hence these elements satisfy (b). The theorem is proved.

5.3. For any $w \in W$ we set $c_{w}=\sum_{y \in W} p_{y, w} T_{y}$ where $p_{y, w} \in \mathcal{A}_{\leq 0}$. By the proof of 5.2 we have

$$
\begin{aligned}
& p_{y, w}=0 \text { unless } y \leq w, \\
& p_{w, w}=1, \\
& p_{y, w} \in \mathcal{A}_{<0} \text { if } y<w .
\end{aligned}
$$

Moreover, for any $x<w$ in $W$ we have

$$
\bar{p}_{x, w}=\sum_{y ; x \leq y \leq w} r_{x, y} p_{y, w} .
$$

Proposition 5.4. (a) Assume that $L(s)>0$ for all $s \in S$. If $x \leq w$, then

$p_{x, w}=v^{-L(w)+L(x)} \bmod v^{-L(w)+L(x)+1} \mathbf{Z}[v]$.

(b) Without assumption on $L$, for $x \leq w$ we have $p_{x, w}=v^{L(w)-L(x)} \mathbf{Z}\left[v^{2}, v^{-2}\right]$.

We prove (a) by induction on $l(w)-l(x)$. If $l(w)-l(x)=0$ then $x=w, p_{x, w}=1$ and the result is obvious. Assume now that $l(w)-l(x)>0$. Using 4.7(b) and the induction hypothesis, we see that $\sum_{y ; x<y \leq w} r_{x, y} p_{y, w}$ is equal to

$$
\sum_{y ; x<y \leq w} \operatorname{sgn}(x) \operatorname{sgn}(y) v^{-L(y)+L(x)} v^{-L(w)+L(y)}=\sum_{y ; x<y \leq w} \operatorname{sgn}(x) \operatorname{sgn}(y) v^{-L(w)+L(x)}
$$

plus strictly higher powers of $v$. Using 4.8, we see that this is $-v^{-L(w)+L(x)}$ plus strictly higher powers of $v$. Thus,

$$
\bar{p}_{x, w}-p_{x, w}=-v^{-L(w)+L(x)}+\text { strictly higher powers of } v \text {. }
$$

Since $\bar{p}_{x, w} \in v \mathbf{Z}[v]$, it is in particular a $\mathbf{Z}$-linear combination of powers of $v$ strictly higher than $-L(w)+L(y)$. Hence

$$
-p_{x, w}=-v^{-L(w)+L(x)}+\text { strictly higher powers of } v \text {. }
$$

This proves (a).

We prove (b) by induction on $l(w)-l(x)$. If $l(w)-l(x)=0$, then $x=w$, $p_{x, w}=1$ and the result is obvious. Assume now that $l(w)-l(x)>0$. Using $4.7(\mathrm{c})$ and the induction hypothesis, we see that

$$
\sum_{y ; x<y \leq w} r_{x, y} p_{y, w} \in \sum_{y ; x<y \leq w} v^{L(y)-L(x)} v^{L(w)-L(y)} \mathbf{Z}\left[v^{2}, v^{-2}\right]=v^{L(w)-L(x)} \mathbf{Z}\left[v^{2}, v^{-2}\right] .
$$

Thus, $\bar{p}_{x, w}-p_{x, w} \in v^{L(w)-L(x)} \mathbf{Z}\left[v^{2}, v^{-2}\right]$. Hence $p_{x, w} \in v^{L(w)-L(x)} \mathbf{Z}\left[v^{2}, v^{-2}\right]$. The proposition is proved. 
5.5. Let $s \in S$. From $T_{s}^{-1}=T_{s}-\left(v_{s}-v_{s}^{-1}\right)$ we see that $r_{1, s}=v_{s}-v_{s}^{-1}$. We also see that

$$
\begin{aligned}
& \overline{T_{s}+v_{s}^{-1}}=T_{s}-\left(v_{s}-v_{s}^{-1}\right)+v_{s}=T_{s}+v_{s}^{-1}, \\
& T_{s}-v_{s}=T_{s}-\left(v_{s}-v_{s}^{-1}\right)-v_{s}^{-1}=T_{s}-v_{s} . \\
& \text { If } L(s)=0 \text { we have } T_{s}^{-1}=T_{s} . \text { Hence, } \\
& \quad c_{s}=T_{s}+v_{s}^{-1} \text { if } L(s)>0, \\
& \quad c_{s}=T_{s}-v_{s} \text { if } L(s)<0, \\
& \quad c_{s}=T_{s} \text { if } L(s)=0 .
\end{aligned}
$$

5.6. Now $h \mapsto h^{b}$ carries $\mathcal{H}_{\leq 0}$ into itself; moreover, it commutes with ${ }^{-}: \mathcal{H} \rightarrow \mathcal{H}$ (as pointed out in 4.9). Hence it carries $c_{w}$ to $c_{w^{-1}}$ for any $w \in W$. It follows that

(a) $p_{y^{-1}, w^{-1}}=p_{y, w}$

for any $y, w \in W$.

\section{LEFT OR RIGHT MULTIPLICATION BY $c_{s}$}

6.1. We preserve the setup of 3.1 and we fix $s \in S$. Assume first that $L(s)=0$. In this case we have $c_{s}=T_{s}$; moreover, for any $y \in W$ we have $T_{s} T_{y}=T_{s y}$. Hence for $w \in W$ we have

$$
c_{s} c_{w}=\sum_{y} p_{y, w} T_{s} T_{y}=\sum_{y} p_{y, w} T_{s y}=\sum_{y} p_{s y, w} T_{y}
$$

We see that $c_{s} c_{w} \in \mathcal{H}_{\leq 0}$ and $c_{s} c_{w}=T_{s w} \bmod \mathcal{H}_{<0}$. Since $\overline{c_{s} c_{w}}=c_{s} c_{w}$, it follows that, in this case, $c_{s} c_{w}=c_{s w}$. Similarly we have $c_{w} c_{s}=c_{w s}$.

6.2. In the remainder of this section (except in 6.8) we assume that $L(s)>0$.

Proposition 6.3. To any $y, w \in W$ such that $s y<y<w<$ sw one can assign uniquely an element $\mu_{y, w}^{s} \in \mathcal{A}$ so that

(i) $\bar{\mu}_{y, w}^{s}=\mu_{y, w}^{s}$ and

(ii) $\sum_{z ; y \leq z<w ; s z<z} p_{y, z} \mu_{z, w}^{s}-v_{s} p_{y, w} \in \mathcal{A}_{<0}$ for any $y, w \in W$ such that $s y<y<w<s w$.

Let $y, w$ be as above. We may assume that $\mu_{z, w}^{s}$ are already defined for all $z$ such that $y<z<w ; s z<z$. Then condition (ii) is of the form:

$\mu_{y, w}^{s}$ equals a known element of $\mathcal{A}$ modulo $\mathcal{A}_{<0}$.

This condition determines uniquely the coefficients of $v^{n}$ with $n \geq 0$ in $\mu_{y, w}^{s}$. Then condition (i) determines uniquely the coefficients of $v^{n}$ with $n<0$ in $\mu_{y, w}^{s}$. The proposition is proved.

Proposition 6.4. Let $y, w \in W$ be such that sy $<y<w<s w$. Then $\mu_{y, w}^{s}$ is a Z-linear combination of powers $v^{n}$ with $-L(s)+1 \leq n \leq L(s)-1$ and $n=L(w)-L(y)-L(s) \bmod 2$.

We may assume that this is already known for all $\mu_{z, w}^{s}$ with $z$ such that $y<$ $z<w ; s z<z$. Using 6.3(ii) and 5.4, we see that $\mu_{y, w}^{s}$ is a Z-linear combination 
of powers $v^{n}$ such that, whenever $n \geq 0$, we have $n \leq L(s)-1$ and $n=L(w)-$ $L(y)-L(s) \bmod 2$. Using now $6.3(\mathrm{i})$, we deduce the remaining assertions of the proposition.

Corollary 6.5. Assume that $L(s)=1$. Let $y, w \in W$ be such that $s y<y<w<$ sw. Then $\mu_{y, w}^{s}$ is an integer, equal to the coefficient of $v^{-1}$ in $p_{y, w}$. In particular, it is 0 unless $L(w)-L(y)$ is odd.

In this case, the inequalities of 6.4 become $0 \leq n \leq 0$. They imply $n=0$. Thus, $\mu_{y, w}^{s} \in \mathbf{Z}$. Picking up the coefficient of $v^{0}$ in the two sides of $6.3(\mathrm{ii})$, we see that $\mu_{y, w}^{s}$ is equal to the coefficient of $v^{-1}$ in $p_{y, w}$. The last assertion follows from 5.4.

Theorem 6.6. Let $w \in W$.

(a) If $w<s w$, then $c_{s} c_{w}=c_{s w}+\sum_{z ; s z<z<w} \mu_{z, w}^{s} c_{z}$.

(b) If $s w<w$, then $c_{s} c_{w}=\left(v_{s}+v_{s}^{-1}\right) c_{w}$.

Since $c_{s}=T_{s}+v_{s}^{-1}$ (see 5.5), we see that (b) is equivalent to $\left(T_{s}-v_{s}\right) c_{w}=0$, or to

(c) $p_{x, w}=v_{s}^{-1} p_{s x, w}$

(where $s w<w, x<s x$ ). We prove the theorem by induction on $l(w)$. If $l(w)=0$, then $w=1$ and the result is obvious. Assume now that $l(w) \geq 1$ and that the result holds when $w$ is replaced by $w^{\prime}$ with $l\left(w^{\prime}\right)<l(w)$.

Case 1. Assume that $w<s w$. Using $c_{s}=T_{s}+v_{s}^{-1}$, we see that the coefficient of $T_{y}$ in the left hand side minus the right hand side of (a) is

$$
f_{y}=v_{s}^{\sigma} p_{y, w}+p_{s y, w}-p_{y, s w}-\sum_{z ; y \leq z<w ; s z<z} p_{y, z} \mu_{z, w}^{s}
$$

where $\sigma=1$ if $s y<y$ and $\sigma=-1$ if $s y>y$. We must show that $f_{y}=0$. We first show that

(d) $f_{y} \in \mathcal{A}_{<0}$.

If $s y<y$ this follows from 6.3(ii). (The contribution of $p_{s y, w}-p_{y, s w}$ is in $\mathcal{A}_{<0}$ if $s y \neq w$ and is $1-1=0$ if $s y=w$.)

If $s y>y$ then, by (c) (applied to $z$ in the sum, instead of $w$ ), we have

$$
\begin{aligned}
f_{y} & =v_{s}^{-1} p_{y, w}+p_{s y, w}-p_{y, s w}-\sum_{z ; y \leq z<w ; s z<z} v_{s}^{-1} p_{s y, z} \mu_{z, w}^{s} \\
& =v_{s}^{-1} f_{s y}+v_{s}^{-1} p_{s y, s w}-p_{y, s w}
\end{aligned}
$$

(the second equality holds by $2.5(\mathrm{a})$ ) and this is in $\mathcal{A}_{<0}$ since $f_{\text {sy }} \in \mathcal{A}_{<0}$ (by the previous paragraph), $v_{s}^{-1} \in \mathcal{A}_{<0}$ and since $y \neq s w$. Thus, (d) is proved.

Since both sides of (a) are fixed by ${ }^{-}$, the sum $\sum_{y} f_{y} T_{y}$ is fixed by ${ }^{-}$. From (d) and $5.2(\mathrm{e})$ we see that $f_{y}=0$ for all $y$, as required. 
Case 2. Assume that $w>s w$. Then case 1 is applicable to $s w$ (by the induction hypothesis). We see that

$$
c_{w}=\left(T_{s}+v_{s}^{-1}\right) c_{s w}-\sum_{z ; s z<z<s w} \mu_{z, s w}^{s} c_{z}
$$

Now $\left(T_{s}-v_{s}\right)\left(T_{s}+v_{s}^{-1}\right)=0$ and $\left(T_{s}-v_{s}\right) c_{z}=0$ for each $z$ in the sum (by the induction hypothesis). Hence $\left(T_{s}-v_{s}\right) c_{w}=0$. The theorem is proved.

Corollary 6.7. Let $w \in W$.

(a) If $w<w s$, then $c_{w} c_{s}=c_{w s}+\sum_{z ; z s<z<w} \mu_{z^{-1}, w^{-1}}^{s} c_{z}$.

(b) If $w s<w$, then $c_{w} c_{s}=\left(v_{s}+v_{s}^{-1}\right) c_{w}$.

We write the equalities in $6.6(\mathrm{a}),(\mathrm{b})$ for $w^{-1}$ instead of $w$ and we apply to these equalities $h \mapsto h^{b}$ which carries $c_{w}$ to $c_{w^{-1}}$; the corollary follows.

6.8. Now $6.3,6.6,6.7$ remain valid when $L(s)<0$ provided that we replace in their statements and proofs $v_{s}$ by $-v_{s}^{-1}$.

\section{Dihedral GROUPS}

7.1. We preserve the setup of 3.1; we assume that $S$ consists of two elements $s_{1}, s_{2}$. For $i=1,2$, let $L_{i}=L\left(s_{i}\right), T_{i}=T_{s_{i}}, c_{i}=c_{s_{i}}$. We assume that $L_{1}>0, L_{2}>0$. Let $\zeta=v^{L_{1}-L_{2}}+v^{L_{2}-L_{1}} \in \mathcal{A}$. Let $m=m_{s_{1}, s_{2}}$. Let $1_{k}, 2_{k}$ be as in 1.4. For $w \in W$ we set

$$
\Gamma_{w}=\sum_{y ; y \leq w} v^{-L(w)+L(y)} T_{y} .
$$

Lemma 7.2. We have

$c_{1} \Gamma_{2_{k}}=\Gamma_{1_{k+1}}+v^{L_{1}-L_{2}} \Gamma_{1_{k-1}}$ if $k \in[2, m)$,

$c_{2} \Gamma_{1_{k}}=\Gamma_{2_{k+1}}+v^{-L_{1}+L_{2}} \Gamma_{2_{k-1}}$ if $k \in[2, m)$,

$c_{1} \Gamma_{2_{k}}=\Gamma_{1_{k+1}}$ if $k=0,1$,

$c_{2} \Gamma_{1_{k}}=\Gamma_{2_{k+1}}$ if $k=0,1$.

Since $c_{i}=T_{i}+v^{-L_{i}}$, the proof is an easy exercise.

Proposition 7.3. Assume that $L_{1}=L_{2}$. For any $w \in W$ we have $c_{w}=\Gamma_{w}$.

This is clear when $l(w) \leq 1$. In the present case Lemma 7.2 gives

$$
\Gamma_{1_{k+1}}=c_{1} \Gamma_{2_{k}}-\Gamma_{1_{k-1}}, \quad \Gamma_{2_{k+1}}=c_{2} \Gamma_{1_{k}}-\Gamma_{2_{k-1}}
$$

for $k \in[1, m)$. This shows by induction on $k$ that $\bar{\Gamma}_{w}=\Gamma_{w}$ for all $w \in W$. Clearly, $\Gamma_{w}=T_{w} \bmod \mathcal{H}_{<0}$. The lemma follows. 
7.4. In 7.4-7.6 we assume that $L_{2}>L_{1}$. In this case, if $m<\infty$, then $m$ is even. (See 3.1.) For $2 k+1 \in[1, m)$ we set

$$
\begin{aligned}
& \Gamma_{2_{2 k+1}}^{\prime}=\sum_{s \in[0, k-1]}\left(1-v^{2 L_{1}}+v^{4 L_{1}}-\cdots+(-1)^{s} v^{2 s L_{1}}\right) v^{-s L_{1}-s L_{2}} \\
& \times\left(T_{2_{2 k-2 s+1}}+v^{-L_{2}} T_{2_{2 k-2 s}}+v^{-L_{2}} T_{1_{2 k-2 s}}+v^{-2 L_{2}} T_{1_{2 k-2 s-1}}\right) \\
& +\left(1-v^{2 L_{1}}+v^{4 L_{1}}-\cdots+(-1)^{k} v^{2 k L_{1}}\right) v^{-k L_{1}-k L_{2}}\left(T_{2_{1}}+v^{-L_{2}} T_{2_{0}}\right) .
\end{aligned}
$$

For $2 k+1 \in[3, m)$ we set

$$
\begin{aligned}
& \Gamma_{1_{2 k+1}}^{\prime}=T_{1_{2 k+1}}+v^{-L_{1}} T_{1_{2 k}}+v^{-L_{1}} T_{2_{2 k}}+v^{-2 L_{1}} T_{2_{2 k-1}} \\
& +\sum_{\substack{y \\
y \leq 1_{2 k-1}}} v^{-L(w)+L(y)}\left(1+v^{2 L_{1}}\right) T_{y}
\end{aligned}
$$

where $w=1_{2 k+1}$. For $w$ such that $l(w)$ is even and for $w=1_{1}$ we set $\Gamma_{w}^{\prime}=\Gamma_{w}$.

Lemma 7.5. We have

(a) $c_{1} \Gamma_{2_{k^{\prime}}}^{\prime}=\Gamma_{1_{k^{\prime}+1}}^{\prime}$, if $k^{\prime} \in[0, m)$;

(b) $c_{2} \Gamma_{1_{k^{\prime}}}^{\prime}=\Gamma_{2_{k^{\prime}+1}}^{\prime}+\zeta \Gamma_{2_{k^{\prime}-1}}^{\prime}+\Gamma_{2_{k^{\prime}-3}}^{\prime}$, if $k^{\prime} \in[4, m)$;

(c) $c_{2} \Gamma_{1_{k^{\prime}}}^{\prime}=\Gamma_{2_{k^{\prime}+1}}^{\prime}+\zeta \Gamma_{2_{k^{\prime}-1}}^{\prime}$, if $k^{\prime}=2,3, k^{\prime}<m$;

(d) $c_{2} \Gamma_{k_{k^{\prime}}}^{\prime}=\Gamma_{2_{k^{\prime}+1}}^{\prime}$ if $k^{\prime}=0,1$.

From the definitions we have

(e) $\Gamma_{2_{2 k+1}}^{\prime}=\sum_{s \in[0, k]}(-1)^{s} v^{s\left(L_{1}-L_{2}\right)} \Gamma_{2_{2 k-2 s+1}}$ if $2 k+1 \in[1, m)$,

(f) $\Gamma_{1_{2 k+1}}^{\prime}=\Gamma_{1_{2 k+1}}+v^{L_{1}-L_{2}} \Gamma_{1_{2 k-1}}$ if $2 k+1 \in[3, m)$.

We prove (a) for $k^{\prime}=2 k+1$. The left hand side can be computed using (e) and 7.2 :

$$
\begin{aligned}
& c_{1} \Gamma_{2_{2 k+1}}^{\prime}=c_{1}\left(\Gamma_{2_{2 k+1}}-v^{L_{1}-L_{2}} \Gamma_{2_{2 k-1}}+v^{2 L_{1}-2 L_{2}} \Gamma_{2_{2 k-3}}+\ldots\right) \\
& =\Gamma_{1_{2 k+2}}+v^{L_{1}-L_{2}} \Gamma_{1_{2 k}}-v^{L_{1}-L_{2}} \Gamma_{1_{2 k}}-v^{2 L_{1}-2 L_{2}} \Gamma_{1_{2 k-2}} \\
& +v^{2 L_{1}-2 L_{2}} \Gamma_{1_{2 k-2}}-v^{3 L_{1}-3 L_{2}} \Gamma_{1_{2 k-4}}+\cdots=\Gamma_{1_{2 k+2}}=\Gamma_{1_{2 k+2}}^{\prime}
\end{aligned}
$$

This proves (a) for $k^{\prime}=2 k+1$. Now (a) for $k^{\prime}=0$ is trivial. We prove (a) for $k^{\prime}=2 k \geq 2$. The left hand side can be computed using 7.2 and (f):

$$
c_{1} \Gamma_{2_{2 k}}^{\prime}=c_{1} \Gamma_{2_{2 k}}=\Gamma_{1_{2 k+1}}+v^{L_{1}-L_{2}} \Gamma_{1_{2 k-1}}=\Gamma_{1_{2 k+1}}^{\prime} .
$$

This proves (a) for $k^{\prime}=2 k$. We prove (b) for $k^{\prime}=2 k$. The left hand side can be computed using 7.2:

$$
c_{2} \Gamma_{1_{2 k}}^{\prime}=c_{2} \Gamma_{1_{2 k}}=\Gamma_{2_{2 k+1}}+v^{-L_{1}+L_{2}} \Gamma_{2_{2 k-1}}
$$


The right hand side of (b) is (using (e)):

$$
\begin{aligned}
& \Gamma_{2_{2 k+1}}-v^{L_{1}-L_{2}} \Gamma_{2_{2 k-1}}+v^{2 L_{1}-2 L_{2}} \Gamma_{2_{2 k-3}}+\ldots \\
& +\zeta \Gamma_{2_{2 k-1}}-v^{L_{1}-L_{2}} \zeta \Gamma_{2_{2 k-3}}+v^{2 L_{1}-2 L_{2}} \zeta \Gamma_{2_{2 k-5}}+\ldots \\
& +\Gamma_{2_{2 k-3}}-v^{L_{1}-L_{2}} \Gamma_{2_{2 k-5}}+v^{2 L_{1}-2 L_{2}} \Gamma_{2_{2 k-7}}+\cdots=\Gamma_{2_{2 k+1}}+v^{-L_{1}+L_{2}} \Gamma_{2_{2 k-1}} .
\end{aligned}
$$

This proves (b) for $k^{\prime}=2 k$. We prove (b) for $k^{\prime}=2 k+1$. The left hand side can be computed using (f) and 7.2:

$$
\begin{aligned}
& c_{2} \Gamma_{1_{2 k+1}}^{\prime}=c_{2}\left(\Gamma_{1_{2 k+1}}+v^{L_{1}-L_{2}} \Gamma_{1_{2 k-1}}\right) \\
& =\Gamma_{2_{2 k+2}}+v^{-L_{1}+L_{2}} \Gamma_{2_{2 k}}+v^{L_{1}-L_{2}} \Gamma_{2_{2 k}}+\Gamma_{2_{2 k-2}}=\Gamma_{2_{2 k+2}}^{\prime}+\zeta \Gamma_{2_{2 k}}^{\prime}+\Gamma_{2_{2 k-2}}^{\prime} .
\end{aligned}
$$

This proves (b) for $k^{\prime}=2 k+1$. The proof of (c),(d) is similar to that of (b). This completes the proof.

Proposition 7.6. For any $w \in W$ we have $c_{w}=\Gamma_{w}^{\prime}$.

Clearly, $\Gamma_{w}^{\prime}=T_{w} \bmod \mathcal{H}_{<0}$. From the formulas in 7.5 we see by induction on $l(w)$ that $\bar{\Gamma}_{w}^{\prime}=\Gamma_{w}^{\prime}$ for all $w$. The proposition is proved. (This was proved for $m=4$ in [L6], for $m=6$ in [X], for general $m$ independently in [L12] and [GP, p.396].)

Proposition 7.7. Assume that $m=\infty$. For $a \in\{1,2\}$, let $f_{a}=v^{L(a)}+v^{-L(a)}$.

(a) If $L_{1}=L_{2}$ and $k, k^{\prime} \geq 0$ then $c_{a_{2 k+1}} c_{a_{2 k^{\prime}+1}}=f_{a} \sum_{u \in\left[0, \min \left(2 k, 2 k^{\prime}\right)\right]} c_{a_{2 k+2 k^{\prime}+1-2 u}}$.

(b) If $L_{2}>L_{1}$ and $k, k^{\prime} \geq 0$ then $c_{2_{2 k+1}} c_{2_{2 k^{\prime}+1}}=f_{2} \sum_{u \in\left[0, \min \left(k, k^{\prime}\right)\right]} c_{2_{2 k+2 k^{\prime}+1-4 u}}$.

(c) If $L_{2}>L_{1}$ and $k, k^{\prime} \geq 1$ then

$$
c_{1_{2 k+1}} c_{1_{2 k^{\prime}+1}}=f_{1} \sum_{u \in\left[0, \min \left(k-1, k^{\prime}-1\right)\right]} p_{u} c_{1_{2 k+2 k^{\prime}+1-2 u}}
$$

where $p_{u}=\zeta$ for $u$ odd, $p_{u} \in \mathbf{Z}$ for $u$ even.

We prove (a). For $k=k^{\prime}=0$ the equality in (a) is clear. Assume now that $k=0, k^{\prime} \geq 1$. Using $7.2,7.3$, we have

$$
\begin{aligned}
& c_{2} c_{2_{2 k^{\prime}+1}}=c_{2}\left(c_{2} c_{1_{2 k^{\prime}}}-c_{2_{2 k^{\prime}-1}}\right)=f_{2} c_{2} c_{1_{2 k^{\prime}}}-f_{2} c_{2} 2_{2 k^{\prime}-1} \\
& =f_{2} c_{2} 2_{2 k^{\prime}+1}+f_{2} c_{2 k^{\prime}-1}-f_{2} c_{2 k^{\prime}-1}=f_{2} c_{2 k^{\prime}+1},
\end{aligned}
$$

as required. We now prove the equality in (a) for fixed $k^{\prime}$, by induction on $k$. The case $k=0$ is already known. Assume now that $k=1$. From 7.2, 7.3 we have $c_{2_{3}}=c_{2} c_{1} c_{2}-c_{2}$. Using this and $7.2,7.3$, we have

$$
\begin{aligned}
& c_{23} c_{2 k^{\prime}+1}=c_{2} c_{1} c_{2} c_{2 k^{\prime}+1}-c_{2} c_{2 k^{\prime}+1}=f_{2} c_{2} c_{1_{2 k^{\prime}+2}}+f_{2} c_{2} c_{1_{2 k^{\prime}}}-f_{2} c_{2_{2 k^{\prime}+1}} \\
& =f_{2} c_{2 k^{\prime}+3}+f_{2} c_{2 k^{\prime}+1}+f_{2} c_{2 k^{\prime}+1}+\left(1-\delta_{k^{\prime}, 0}\right) f_{2} c_{2 k^{\prime}-1}-f_{2} c_{2 k^{\prime}+1} \\
& =f_{2} c_{2 k^{\prime}+3}+f_{2} c_{2 k^{\prime}+1}+f_{2}\left(1-\delta_{k^{\prime}, 0}\right) c_{2_{2 k^{\prime}-1}} \text {, }
\end{aligned}
$$


as required. Assume now that $k \geq 2$. From $7.2,7.3$ we have

$$
c_{2_{2 k+1}}=c_{2} c_{1} c_{2_{2 k-1}}-2 c_{2_{2 k-1}}-c_{2_{2 k-3}} .
$$

Using this and the induction hypothesis we have

$$
\begin{aligned}
& c_{2 k+1} c_{2}{ }_{2 k^{\prime}+1}=c_{2} c_{1} c_{2 k-1} c_{2} c_{2 k^{\prime}+1}-2 c_{2 k-1} c_{2} c_{2 k^{\prime}+1}-c_{2 k-3} c_{2} c_{2 k^{\prime}+1} \\
& =f_{2} c_{1} c_{2} \sum_{u \in\left[0, \min \left(2 k-2, k^{\prime}\right)\right]} c_{2_{2 k+2 k^{\prime}-1-2 u}}-f_{2} \sum_{u \in\left[0, \min \left(2 k-2, k^{\prime}\right)\right]} c_{2_{2 k+2 k^{\prime}-1-2 u}} \\
& -f_{2} \sum_{u \in\left[0, \min \left(2 k-4, k^{\prime}\right)\right]} c_{2 k+2 k^{\prime}-3-2 u} .
\end{aligned}
$$

We now use 7.2,7.3 and (a) follows (for $a=2$ ). The case $a=1$ is similar.

We prove (b). For $k=k^{\prime}=0$ the equality in (b) is clear. Assume now that $k=0, k^{\prime}=1$. Using $7.5,7.6$, we have

$$
c_{2} c_{2_{3}}=c_{2}\left(c_{2} c_{1_{2}}-\zeta c_{2_{1}}\right)=f_{2} c_{2} c_{1_{2}}-f_{2} \zeta c_{2_{1}}=f_{2} c_{2_{3}}+f_{2} \zeta c_{2_{1}}-f_{2} \zeta c_{2_{1}}=f_{2} c_{2_{3}} \text {, }
$$

as required. Assume next that $k=0, k^{\prime} \geq 2$. Using 7.5, 7.6, we have

$$
\begin{aligned}
& c_{2} c_{2_{2 k^{\prime}+1}}=c_{2}\left(c_{2} c_{1_{2 k^{\prime}}}-\zeta c_{2_{2 k^{\prime}-1}}-c_{2_{2 k^{\prime}-3}}\right)=f_{2} c_{2} c_{1_{2 k^{\prime}}}-f_{2} \zeta c_{2_{2 k^{\prime}-1}}-f_{2} c_{2_{2 k^{\prime}-3}} \\
& =f_{2} c_{2_{2 k^{\prime}+1}}+f_{2} \zeta c_{2_{2 k^{\prime}-1}}+f_{2} c_{2_{2 k^{\prime}-3}}-f_{2} \zeta c_{2_{2 k^{\prime}-1}}-f_{2} \zeta c_{2_{2 k^{\prime}-3}}=f_{2} c_{2_{2 k^{\prime}+1}},
\end{aligned}
$$

as required. We now prove the equality in (a) for fixed $k^{\prime}$, by induction on $k$. The case $k=0$ is already known. Assume now that $k=1$. From 7.5, 7.6 we have $c_{2_{3}}=c_{2} c_{1} c_{2}-\zeta c_{2}$. Using this and $7.5,7.6$, we have

$$
\begin{aligned}
& c_{23} c_{2} 2_{2 k^{\prime}+1}=c_{2} c_{1} c_{2} c_{2 k^{\prime}+1}-\zeta c_{2} c_{2 k^{\prime}+1}=f_{2} c_{2} c_{1_{2 k^{\prime}+2}}-f_{2} \zeta c_{2 k^{\prime}+1} \\
& =f_{2} c_{2 k^{\prime}+3}+f_{2} \zeta c_{2 k^{\prime}+1}+\left(1-\delta_{k^{\prime}, 0}\right) f_{2} c_{2 k^{\prime}-1}-f_{2} \zeta c_{2 k^{\prime}+1} \\
& =f_{2} c_{2 k^{\prime}+3}+\left(1-\delta_{k^{\prime}, 0}\right) f_{2} c_{2}+2_{2 k^{\prime}-1}
\end{aligned}
$$

as required. Assume now that $k \geq 2$. From 7.5, 7.6 we have

$$
c_{2_{2 k+1}}=c_{2} c_{1} c_{2 k-1}-\zeta c_{2_{2 k-1}}-c_{2_{2 k-3}} .
$$

Using this and the induction hypothesis we have

$$
\begin{aligned}
& c_{2_{2 k+1}} c_{2_{2 k^{\prime}+1}}=c_{2} c_{1} c_{2 k+1} c_{2_{2 k^{\prime}+1}}-\zeta c_{2 k-1} c_{2} c_{2 k^{\prime}+1}-c_{2 k-3} c_{2 k^{\prime}+1} \\
& =f_{2} c_{2} c_{1} \sum_{u \in\left[0, \min \left(k-1, k^{\prime}\right)\right]} c_{2_{2 k+2 k^{\prime}-1-4 u}}-f_{2} \zeta \sum_{u \in\left[0, \min \left(k-1, k^{\prime}\right)\right]} c_{2_{2 k+2 k^{\prime}-1-4 u}} \sum_{u \in\left[0, \min \left(k-2, k^{\prime}\right)\right]} c_{2_{2 k+2 k^{\prime}-3-4 u}} .
\end{aligned}
$$

We now use 7.5, 7.6 and (b) follows.

The proof of (c) is similar to that of (b). This completes the proof. 
Proposition 7.8. Assume that $4 \leq m<\infty$ and $L_{2}>L_{1}$, so that $m=2 k+2$ with $k \geq 1$. Let

$$
p_{0}=(-1)^{k}\left(v^{L_{2}}+v^{-L_{2}}\right)\left(v^{k\left(L_{2}-L_{1}\right)}+v^{(k-2)\left(L_{2}-L_{1}\right)}+\cdots+v^{-k\left(L_{2}-L_{1}\right)}\right) .
$$

Then

$$
c_{2_{m-1}} c_{2_{m-1}}=p c_{2_{m-1}}+q c_{2_{m}}
$$

for some $p, q \in \mathcal{A}$. Moreover, $p=p_{0}$.

From 7.5, 7.6, we see that $\mathcal{A} c_{2_{m-1}}+\mathcal{A} c_{2_{m}}$ is a two-sided ideal of $\mathcal{H}$. Hence (a) holds for some (unknown) $p, q \in \mathcal{A}$. It remains to compute $p$. Define an algebra homomorphism $\chi: \mathcal{H} \rightarrow \mathcal{A}$ by $\chi\left(T_{1}\right)=-v^{-L_{1}}, \chi\left(T_{2}\right)=v^{L_{2}}$. Since $c_{2_{m}}=\left(T_{1}+v^{-L_{1}}\right) h$ for some $h \in \mathcal{H}$ (see 7.5,7.6) we see that $\chi\left(c_{2_{m}}\right)=0$. Hence applying $\chi$ to (a) gives $\chi\left(c_{2_{m-1}}\right)^{2}=p \chi\left(c_{2_{m-1}}\right)$. It is thus enough to show that $\chi\left(c_{2_{m-1}}\right)=p_{0}$. We verify this for $m=4$ :

$$
\begin{aligned}
& \chi\left(T_{2} T_{1} T_{2}+v^{-L_{2}} T_{2} T_{1}+v^{-L_{2}} T_{1} T_{2}+v^{-2 L_{2}} T_{1}\right. \\
& \left.+\left(v^{-L_{1}-L_{2}}-v^{L_{1}-L_{2}}\right) T_{2}+\left(v^{-L_{1}-2 L_{2}}-v^{L_{1}-2 L_{2}}\right)\right) \\
& =-v^{-L_{1}+2 L_{2}}-2 v^{-L_{1}}-v^{-L_{1}-2 L_{2}}+\left(v^{-L_{1}-L_{2}}-v^{L_{1}-L_{2}}\right) v^{L_{2}} \\
& +v^{-L_{1}-2 L_{2}}-v^{L_{1}-2 L_{2}}=-v^{-L_{1}+2 L_{2}}-v^{-L_{1}}-v^{L_{1}}-v^{L_{1}-2 L_{2}}
\end{aligned}
$$

and for $m=6$ :

$$
\begin{aligned}
& \chi\left(T_{2} T_{1} T_{2} T_{1} T_{2}+v^{-L_{2}} T_{2} T_{1} T_{2} T_{1}+v^{-L_{2}} T_{1} T_{2} T_{1} T_{2}+v^{-2 L_{2}} T_{1} T_{2} T_{1}\right. \\
& +\left(v^{-L_{1}-L_{2}}-v^{L_{1}-L_{2}}\right) T_{2} T_{1} T_{2}+\left(v^{-L_{1}-2 L_{2}}-v^{L_{1}-2 L_{2}}\right) T_{1} T_{2} \\
& +\left(v^{-L_{1}-2 L_{2}}-v^{L_{1}-2 L_{2}}\right) T_{2} T_{1}+\left(v^{-L_{1}-3 L_{2}}-v^{L_{1}-3 L_{2}}\right) T_{1} \\
& \left.+\left(v^{-2 L_{1}-2 L_{2}}-v^{-2 L_{2}}+v^{2 L_{1}-2 L_{2}}\right) T_{2}+\left(v^{-2 L_{1}-3 L_{2}}-v^{-3 L_{2}}+v^{2 L_{1}-3 L_{2}}\right)\right) \\
& =v^{-2 L_{1}+3 L_{2}}+2 v^{-2 L_{1}+L_{2}}+v^{-2 L_{1}-L_{2}}-v^{-2 L_{1}+L_{2}}-2 v^{-2 L_{1}-L_{2}}-v^{-2 L_{1}-3 L_{2}}+v^{L_{2}} \\
& +2 v^{-L_{2}}+v^{-3 L_{2}}+v^{-2 L_{1}-L_{2}}-v^{-L_{2}}+v^{2 L_{1}-L_{2}}+v^{-2 L_{1}-3 L_{2}}-v^{-3 L_{2}}+v^{2 L_{1}-3 L_{2}} \\
& =v^{-2 L_{1}+3 L_{2}}+v^{-2 L_{1}+L_{2}}+v^{L_{2}}+v^{-L_{2}}+v^{2 L_{1}-L_{2}}+v^{2 L_{1}-3 L_{2}} .
\end{aligned}
$$

Analogous computations can be carried out for any even $m$. The proposition is proved.

\section{Cells}

8.1. We preserve the setup of 3.1. For $z \in W$ define $D_{z} \in \operatorname{Hom}_{\mathcal{A}}(\mathcal{H}, \mathcal{A})$ by $D_{z}\left(c_{w}\right)=\delta_{z, w}$ for all $w \in W$. For $w, w^{\prime} \in W$ we write

$w \leftarrow \mathcal{L} w^{\prime}\left(\right.$ or $\left.w^{\prime} \rightarrow_{\mathcal{L}} w\right)$ if $D_{w}\left(c_{s} c_{w^{\prime}}\right) \neq 0$ for some $s \in S$;

$w \leftarrow_{\mathcal{R}} w^{\prime}\left(\right.$ or $\left.w^{\prime} \rightarrow_{\mathcal{R}} w\right)$ if $D_{w}\left(c_{w^{\prime}} c_{s}\right) \neq 0$ for some $s \in S$.

If $w, w^{\prime} \in W$, we say that $w \leq_{\mathcal{L}} w^{\prime}\left(\right.$ resp. $\left.w \leq_{\mathcal{R}} w^{\prime}\right)$ if there exist $w=$ 
$w_{0}, w_{1}, \ldots, w_{n}=w^{\prime}$ in $W$ such that for any $i \in[0, n-1]$ we have $w_{i} \leftarrow \mathcal{L} w_{i+1}$ (resp. $w_{i} \leftarrow \mathcal{R} w_{i+1}$ ).

If $w, w^{\prime} \in W$, we say that $w \leq_{\mathcal{L} \mathcal{R}} w^{\prime}$ if there exist $w=w_{0}, w_{1}, \ldots, w_{n}=w^{\prime}$ in $W$ such that for any $i \in[0, n-1]$ we have either $w_{i} \leftarrow_{\mathcal{L}} w_{i+1}$ or $w_{i} \leftarrow_{\mathcal{R}} w_{i+1}$.

Clearly $\leq_{\mathcal{L}}, \leq_{\mathcal{R}}, \leq_{\mathcal{L} \mathcal{R}}$ are preorders on $W$. Let $\sim_{\mathcal{L}}, \sim_{\mathcal{R}}, \sim_{\mathcal{L} \mathcal{R}}$ be the associated equivalence relations. (For example, we have $w \sim_{\mathcal{L}} w^{\prime}$ if and only if $w \leq_{\mathcal{L}} w^{\prime}$ and $w^{\prime} \leq_{\mathcal{L}} w$.) The equivalence classes on $W$ for $\sim_{\mathcal{L}}, \sim_{\mathcal{R}}, \sim_{\mathcal{L} \mathcal{R}}$ are called respectively left cells, right cells, two-sided cells of $W$. They depend on the weight function $L$.

If $w, w^{\prime} \in W$, we say that $w<_{\mathcal{L}} w^{\prime}$ (resp. $\left.w<_{\mathcal{R}} w^{\prime} ; w<_{\mathcal{L} \mathcal{R}} w^{\prime}\right)$ if $w \leq_{\mathcal{L}} w^{\prime}$ and $w \chi_{\mathcal{L}} w^{\prime}$ (resp. $w \leq_{\mathcal{R}} w^{\prime}$ and $w \chi_{\mathcal{R}} w^{\prime} ; w \leq_{\mathcal{L R}} w^{\prime}$ and $\left.w \chi_{\mathcal{L R}} w^{\prime}\right)$.

For $w, w^{\prime} \in W$ we have $w \leq_{\mathcal{L}} w^{\prime} \Leftrightarrow w^{-1} \leq_{\mathcal{R}} w^{\prime-1}$ and $w \leq_{\mathcal{L} \mathcal{R}} w^{\prime} \Leftrightarrow w^{-1} \leq_{\mathcal{L} \mathcal{R}}$ $w^{\prime-1}$.

Hence $w \mapsto w^{-1}$ carries left cells to right cells, right cells to left cells and two-sided cells to two-sided cells.

Lemma 8.2. Let $w^{\prime} \in W$.

(a) $\mathcal{H}_{\leq_{\mathcal{L}} w^{\prime}}=\oplus_{w ; w} \leq_{\mathcal{L}} w^{\prime} \mathcal{A} c_{w}$ is a left ideal of $\mathcal{H}$.

(b) $\mathcal{H}_{\leq_{\mathcal{R}} w^{\prime}}=\oplus_{w ; w \leq_{\mathcal{R}} w^{\prime}} \mathcal{A} c_{w}$ is a right ideal of $\mathcal{H}$.

(c) $\mathcal{H}_{\leq_{\mathcal{L R}} w^{\prime}}=\bigoplus_{w ; w} \leq_{\mathcal{L} \mathcal{R}} w^{\prime} \mathcal{A} c_{w}$ is a two-sided ideal of $\mathcal{H}$.

This follows from the definitions since $c_{s}(s \in S)$ generate $\mathcal{H}$ as an $\mathcal{A}$-algebra.

8.3. Let $Y$ be a left cell of $W$. From 8.3(a) we see that for $y \in Y$,

$$
\oplus_{w ; w \leq} y \mathcal{A} c_{w} / \oplus_{w ; w<{ }_{\mathcal{L}}} \mathcal{A} c_{w}
$$

is a quotient of two left ideals of $\mathcal{H}$ (independent of the choice of $y$ ) hence it is naturally a left $\mathcal{H}$-module; it has an $\mathcal{A}$-basis consisting of the images of $c_{w}(w \in Y)$.

Similarly, if $Y^{\prime}$ is a right cell of $W$ then, for $y^{\prime} \in Y^{\prime}$,

$$
\oplus_{w ; w \leq_{\mathcal{R}} y^{\prime}} \mathcal{A} c_{w} / \oplus_{w ; w<\mathcal{R}} y^{\prime} \mathcal{A} c_{w}
$$

is a quotient of two right ideals of $\mathcal{H}$ (independent of the choice of $y^{\prime}$ ) hence it is naturally a right $\mathcal{H}$-module; it has an $\mathcal{A}$-basis consisting of the images of $c_{w}\left(w \in Y^{\prime}\right)$.

If $Y^{\prime \prime}$ is a two-sided cell of $W$ then, for $y^{\prime \prime} \in Y^{\prime \prime}$,

$$
\oplus_{w ; w \leq_{\mathcal{L R}} y^{\prime \prime}} \mathcal{A} c_{w} / \oplus_{w ; w<_{\mathcal{L} \mathcal{R}} y^{\prime \prime}} \mathcal{A} c_{w}
$$

is a quotient of two two-sided ideals of $\mathcal{H}$ (independent of the choice of $y^{\prime \prime}$ ) hence it is naturally a $\mathcal{H}$-bimodule; it has an $\mathcal{A}$-basis consisting of the images of $c_{w}(w \in$ $\left.Y^{\prime \prime}\right)$.

Lemma 8.4. Let $s \in S$. Assume that $L(s)>0$. Let $\mathcal{H}^{s}=\oplus_{w ; s w<w} \mathcal{A} c_{w},{ }^{s} \mathcal{H}=$ $\oplus_{w ; w s<w} \mathcal{A} c_{w}$.

(a) $\left\{h \in \mathcal{H} ;\left(c_{s}-v_{s}-v_{s}^{-1}\right) h=0\right\}=\mathcal{H}^{s}$. Hence $\mathcal{H}^{s}$ is a right ideal of $\mathcal{H}$. 
(b) $\left\{h \in \mathcal{H} ; h\left(c_{s}-v_{s}-v_{s}^{-1}\right)=0\right\}={ }^{s} \mathcal{H}$. Hence ${ }^{s} \mathcal{H}$ is a left ideal of $\mathcal{H}$.

We prove the equality in (a). If $h \in \mathcal{H}^{s}$ then $\left(c_{s}-v_{s}-v_{s}^{-1}\right) h=0$ by $6.6(\mathrm{~b})$. Conversely, by 6.6, we have $c_{s} h \in \mathcal{H}^{s}$ for any $h \in \mathcal{H}$. Hence, if $h \in \mathcal{H}$ is such that $c_{s} h=\left(v_{s}+v_{s}^{-1}\right) h$, then $\left(v_{s}+v_{s}^{-1}\right) h \in \mathcal{H}^{s}$ so that $h \in \mathcal{H}^{s}$ (since $\mathcal{H} / \mathcal{H}^{s}$ is a free $\mathcal{A}$-module). This proves (a). The proof of (b) is entirely similar. The lemma is proved.

8.5. For $w \in W$ we set $\mathcal{L}(w)=\{s \in S ; s w<w\}, \mathcal{R}(w)=\{s \in S ; w s<w\}$.

Lemma 8.6. Let $w, w^{\prime} \in W$. Assume that $L(s)>0$ for all $s \in S$.

(a) If $w \leq_{\mathcal{L}} w^{\prime}$, then $\mathcal{R}\left(w^{\prime}\right) \subset \mathcal{R}(w)$. If $w \sim_{\mathcal{L}} w^{\prime}$, then $\mathcal{R}\left(w^{\prime}\right)=\mathcal{R}(w)$.

(b) If $w \leq_{\mathcal{R}} w^{\prime}$, then $\mathcal{L}\left(w^{\prime}\right) \subset \mathcal{L}(w)$. If $w \sim_{\mathcal{R}} w^{\prime}$, then $\mathcal{L}\left(w^{\prime}\right)=\mathcal{L}(w)$.

To prove the first assertion of (a), we may assume that $D_{w}\left(c_{s} c_{w^{\prime}}\right) \neq 0$ for some $s \in S$. In this case, let $t \in \mathcal{R}\left(w^{\prime}\right)$. We must prove that $t \in \mathcal{R}(w)$. We have $c_{w^{\prime}} \in{ }^{t} \mathcal{H}$. By 8.4, ${ }^{t} \mathcal{H}$ is a left ideal of $\mathcal{H}$. Hence $c_{s} c_{w^{\prime}} \in{ }^{t} \mathcal{H}$. By the definition of ${ }^{t} \mathcal{H}$, for $h \in{ }^{t} \mathcal{H}$ we have $D_{w}(h)=0$ unless $w t<w$. Hence from $D_{w}\left(c_{s} c_{w^{\prime}}\right) \neq 0$ we deduce $w t<w$, as required. This proves the first assertion of (a). The second assertion of (a) follows immediately from the first. The proof of (b) is entirely similar to that of (a). The lemma is proved.

8.7. In the remainder of this section we write $\leftarrow, \rightarrow$ instead of $\leftarrow \mathcal{L}, \rightarrow_{\mathcal{L}}$. We describe the left cells of $W$ in the setup of 7.3. From 7.2 and 7.3 we can determine all pairs $y \neq w$ such that $y \leftarrow w$

$$
\begin{aligned}
& 1_{0} \rightarrow 2_{1} \leftrightarrows 1_{2} \leftrightarrows 2_{3} \leftrightarrows \ldots \\
& 2_{0} \rightarrow 1_{1} \leftrightarrows 2_{2} \leftrightarrows 1_{3} \leftrightarrows \ldots,
\end{aligned}
$$

if $m=\infty$,

$1_{0} \rightarrow 2_{1} \leftrightarrows 1_{2} \leftrightarrows 2_{3} \leftrightarrows \ldots \leftrightarrows 2_{m-1} \rightarrow 1_{m}$

$2_{0} \rightarrow 1_{1} \leftrightarrows 2_{2} \leftrightarrows 1_{3} \leftrightarrows \ldots \leftrightarrows 1_{m-1} \rightarrow 2_{m}$

if $m<\infty, m$ even,

$1_{0} \rightarrow 2_{1} \leftrightarrows 1_{2} \leftrightarrows 2_{3} \leftrightarrows \ldots \leftrightarrows 1_{m-1} \rightarrow 2_{m}$

$2_{0} \rightarrow 1_{1} \leftrightarrows 2_{2} \leftrightarrows 1_{3} \leftrightarrows \ldots \leftrightarrows 2_{m-1} \rightarrow 1_{m}$

if $m<\infty, m$ odd. Hence the left cells are

$\left\{1_{0}\right\},\left\{2_{1}, 1_{2}, 2_{3}, \ldots\right\},\left\{1_{1}, 2_{2}, 1_{3}, \ldots\right\}$,

if $m=\infty$,

$\left\{1_{0}\right\},\left\{2_{1}, 1_{2}, 2_{3}, \ldots, 2_{m-1}\right\},\left\{1_{1}, 2_{2}, 1_{3}, \ldots, 1_{m-1}\right\},\left\{2_{m}\right\}$,

if $m<\infty, m$ even,

$\left\{1_{0}\right\},\left\{2_{1}, 1_{2}, 2_{3}, \ldots, 1_{m-1}\right\},\left\{1_{1}, 2_{2}, 1_{3}, \ldots, 2_{m-1}\right\},\left\{2_{m}\right\}$,

if $m<\infty, m$ odd.

The two-sided cells are $\left\{1_{0}\right\}, W-\left\{1_{0}\right\}$ if $m=\infty$ and $\left\{1_{0}\right\},\left\{2_{m}\right\}, W-\left\{1_{0}, 2_{m}\right\}$ if $m<\infty$.

8.8. We describe the left cells of $W$ in the setup of 7.4. From 7.5 and 7.6 we can determine all pairs $y \neq w$ such that $y \leftarrow w$. If $m=\infty$, these pairs are:

$$
1_{0} \rightarrow 2_{1} \leftrightarrows 1_{2} \rightarrow 2_{3} \leftrightarrows 1_{4} \rightarrow \ldots, \quad 2_{0} \rightarrow 1_{1} \rightarrow 2_{2} \leftrightarrows 1_{3} \rightarrow 2_{4} \leftrightarrows \ldots
$$


and $2_{1} \leftarrow 1_{4}, 2_{2} \leftarrow 1_{5}, 2_{3} \leftarrow 1_{6}, \ldots$

If $m=4$, these pairs are:

$$
1_{0} \rightarrow 2_{1} \leftrightarrows 1_{2} \rightarrow 2_{3} \rightarrow 1_{4}, \quad 2_{0} \rightarrow 1_{1} \rightarrow 2_{2} \leftrightarrows 1_{3} \rightarrow 2_{4}
$$

If $m=6$, these pairs are:

$$
1_{0} \rightarrow 2_{1} \leftrightarrows 1_{2} \rightarrow 2_{3} \leftrightarrows 1_{4} \rightarrow 2_{5} \rightarrow 1_{6}, \quad 2_{0} \rightarrow 1_{1} \rightarrow 2_{2} \leftrightarrows 1_{3} \rightarrow 2_{4} \leftrightarrows 1_{5} \rightarrow 2_{6}
$$

and $2_{1} \leftarrow 1_{4}, 2_{2} \leftarrow 1_{5}$. An analogous pattern holds for any even $m$.

Hence the left cells are

$$
\left\{1_{0}\right\},\left\{2_{1}, 1_{2}, 2_{3}, 1_{4}, \ldots\right\},\left\{1_{1}\right\},\left\{2_{2}, 1_{3}, 2_{4}, 1_{5}, \ldots\right\}
$$

if $m=\infty$,

$$
\left\{1_{0}\right\},\left\{2_{1}, 1_{2}, 2_{3}, \ldots, 1_{m-2}\right\},\left\{2_{m-1}\right\},\left\{1_{1}\right\},\left\{2_{2}, 1_{3}, 2_{4}, \ldots, 1_{m-1}\right\},\left\{2_{m}\right\}
$$

if $m<\infty$.

The two-sided cells are

$\left\{1_{0}\right\},\left\{1_{1}\right\}, W-\left\{1_{0}, 1_{1}\right\}$, if $m=\infty$ and

$\left\{1_{0}\right\},\left\{1_{1}\right\},\left\{2_{m-1}\right\},\left\{2_{m}\right\}, W-\left\{1_{0}, 1_{1}, 2_{m-1}, 2_{m}\right\}$, if $m<\infty$.

8.9. For further examples of cells (in the case where $L$ is non-costant) see [L3], $[\mathrm{B}],[\mathrm{G}]$.

\section{Cosets of parabolic subgroups}

We preserve the setup of 3.1 .

Lemma 9.1. Let $w \in W$. Assume that $w=s_{1} s_{2} \ldots s_{q}$ with $s_{i} \in S$. We can find a subsequence $i_{1}<i_{2}<\cdots<i_{r}$ of $1,2, \ldots, r$ such that $w=s_{i_{1}} s_{i_{2}} \ldots s_{i_{r}}$ is a reduced expression in $W$.

We argue by induction on $q$. If $q=0$ the result is obvious. Assume that $q>0$. Using the induction hypothesis we can assume that $s_{2} \ldots s_{q}$ is a reduced expression. If $s_{1} s_{2} \ldots s_{q}$ is a reduced expression, we are done. Hence we may assume that $s_{1} s_{2} \ldots s_{q}$ is not a reduced expression. Then $l(w)=q-1$. By 1.7, we can find $j \in[2, q]$ such that $s_{1} s_{2} \ldots s_{j-1}=s_{2} s_{3} \ldots s_{j}$. Then $w=s_{2} s_{3} \ldots s_{j-1} s_{j+1} \ldots s_{q}$ is a reduced expression. The lemma is proved.

9.2. Let $w \in W$. Let $w=s_{1} s_{2} \ldots s_{q}$ be a reduced expression of $w$. Using 1.9 , we see that the set $\left\{s \in S ; s=s_{i}\right.$ for some $\left.i \in[1, q]\right\}$ is independent of the choice of reduced expression. We denote it by $S_{w}$. 
9.3. In the remainder of this section we fix $I \subset S$. Recall that $W_{I}=\langle I\rangle$.

If $w \in W_{I}$ then we can find a reduced expression $w=s_{1} s_{2} \ldots s_{q}$ in $W$ with all $s_{i} \in I$ (we first write $w=s_{1} s_{2} \ldots s_{q}$, a not necessarily reduced expression with all $s_{i} \in I$, and then we apply 9.1). Thus, $S_{w} \subset I$. Conversely, it is clear that if $w^{\prime} \in W$ satisfies $S_{w^{\prime}} \subset I$ then $w^{\prime} \in W_{I}$. It follows that

$W_{I}=\left\{w \in W ; S_{w} \subset I\right\}$.

9.4. Replacing $S,\left(m_{s, s^{\prime}}\right)_{\left(s, s^{\prime}\right) \in S \times S}$ by $I,\left(m_{s, s^{\prime}}\right)_{\left(s, s^{\prime}\right) \in I \times I}$ in the definition of $W$ we obtain a Coxeter group denoted by $W_{I}^{*}$. We have an obvious homomorphism $f: W_{I}^{*} \rightarrow W_{I}$ which takes $s$ to $s$ for $s \in I$.

Proposition 9.5. $f: W_{I}^{*} \rightarrow W_{I}$ is an isomorphism.

We define $f^{\prime}: W_{I} \rightarrow W_{I}^{*}$ as follows: for $w \in W_{I}$ we choose a reduced expression $w=s_{1} s_{2} \ldots s_{q}$ in $W$; then $s_{i} \in I$ for all $i$ (see 9.3) and we set $f^{\prime}(w)=s_{1} s_{2} \ldots s_{q}$ (product in $W_{I}^{*}$ ). This map is well defined. Indeed, if $s_{1}^{\prime} s_{2}^{\prime} \ldots s_{q}^{\prime}$ is another reduced expression for $w$ with all $s_{i} \in I$, then we can pass from $\left(s_{1}, s_{2}, \ldots, s_{q}\right)$ to $\left(s_{1}^{\prime}, s_{2}^{\prime}, \ldots, s_{q}^{\prime}\right)$ by moving along edges of the graph $X$ (see 1.9); but each edge involved in this move will necessarily involve only pairs $\left(s, s^{\prime}\right)$ in $I$, hence the equation $s_{1} s_{2} \ldots s_{q}=s_{1}^{\prime} s_{2}^{\prime} \ldots s_{q}^{\prime}$ must hold in $W_{I}^{*}$. It is clear that $f f^{\prime}(w)=w$ for all $w \in W_{I}$. Hence $f^{\prime}$ is injective.

We show that $f^{\prime}$ is a group homomorphism. It suffices to show that $f^{\prime}(s w)=$ $f^{\prime}(s) f^{\prime}(w)$ for any $w \in W_{I}, s \in I$. This is clear if $l(s w)=l(w)+1$ (in $W$ ). Assume now that $l(s w)=l(w)-1$ (in $W)$. Let $w=s_{1} s_{2} \ldots s_{r}$ be a reduced expression in $W$. Then $s_{i} \in I$ for all $i$. By 1.7 we have (in $W$ ) $s w=s_{1} s_{2} \ldots s_{i-1} s_{i+1} \ldots s_{q}$ for some $i \in[1, q]$. Since $s s_{1} s_{2} \ldots s_{i-1} s_{i+1} \ldots s_{q}$ is a reduced expression for $w$ in $W$, we have $f^{\prime}(w)=s s_{1} s_{2} \ldots s_{i-1} s_{i+1} \ldots s_{q}$ (product in $W_{I}^{*}$ ). We also have $f^{\prime}(w)=$ $s_{1} s_{2} \ldots s_{q}$ (product in $\left.W_{I}^{*}\right)$. Hence $s_{1} s_{2} \ldots s_{i-1} s_{i+1} \ldots s_{q}=s_{1} s_{2} \ldots s_{q}$ (in $\left.W_{I}^{*}\right)$. Hence $s_{1} s_{2} \ldots s_{i-1} s_{i+1} \ldots s_{q}=s s_{1} s_{2} \ldots s_{q}\left(\right.$ in $\left.W_{I}^{*}\right)$. Hence $f^{\prime}(s w)=f^{\prime}(s) f^{\prime}(w)$, as required.

Since the image of $f^{\prime}$ contains the generators $s \in I$ of $W_{I}^{*}$ and $f^{\prime}$ is a group homomorphism, it follows that $f^{\prime}$ is surjective. Hence $f^{\prime}$ is bijective. Since $f f^{\prime}=1$ it follows that $f$ is bijective. The proposition is proved.

9.6. We identify $W_{I}^{*}$ and $W_{I}$ via $f$. Thus, $W_{I}$ is naturally a Coxeter group. Let $l_{I}: W_{I} \rightarrow \mathbf{N}$ be the length function of this Coxeter group. Let $w \in W_{I}$. Let $w=s_{1} s_{2} \ldots s_{q}$ be a reduced expression of $w$ (in $W$ ). Then $s_{i} \in I$ for all $i$ (see 9.3). Hence $l_{I}(w) \leq l(w)$. The reverse inequality $l(w) \leq l_{I}(w)$ is obvious. Hence $l_{I}(w)=l(w)$.

From 2.4 we see that the partial order on $W_{I}$ defined in the same way as $\leq$ on $W$ is just the restriction of $\leq$ from $W$ to $W_{I}$.

Lemma 9.7. Let $W_{I}$ a be a coset in $W$.

(a) This coset has a unique element $w$ of minimal length.

(b) If $y \in W_{I}$ then $l(y w)=l(y)+l(w)$. 
(c) $w$ is characterized by the property that $l(s w)>l(w)$ for all $s \in I$.

Let $w$ be an element of minimal length in the coset. Let $w=s_{1} s_{2} \ldots s_{q}$ be a reduced expression. Let $y \in W_{I}$ and let $y=s_{1}^{\prime} s_{2}^{\prime} \ldots s_{p}^{\prime}$ be a reduced expression in $W_{I}$. Then $y w=s_{1}^{\prime} s_{2}^{\prime} \ldots s_{p}^{\prime} s_{1} s_{2} \ldots s_{q}$. By 9.1 we can drop some of the factors in the last product so that we are left with a reduced expression for $y w$. The factors dropped cannot contain any among the last $q$ since otherwise we would find an element in $W_{I}$ a of strictly smaller length than $w$. Thus, we can find a subsequence $i_{1}<i_{2}<\cdots<i_{r}$ of $1,2, \ldots, p$ such that $y w=s_{i_{1}}^{\prime} s_{i_{2}}^{\prime} \ldots s_{i_{r}}^{\prime} s_{1} s_{2} \ldots s_{q}$ is a reduced expression. It follows that $y=s_{1}^{\prime} s_{2}^{\prime} \ldots s_{p}^{\prime}=s_{i_{1}}^{\prime} s_{i_{2}}^{\prime} \ldots s_{i_{r}}^{\prime}$. Since $p=l(y)$, we must have $r=p$ so that $s_{1}^{\prime} s_{2}^{\prime} \ldots s_{p}^{\prime} s_{1} s_{2} \ldots s_{q}$ is a reduced expression and $l(y w)=p+q=l(y)+l(w)$.

If now $w^{\prime}$ is another element of minimal length in $W_{I} a$ then $w^{\prime}=y w$ for some $y \in W_{I}$. We have $l(w)=l\left(w^{\prime}\right)=l(y)+l(w)$ hence $l(y)=0$ hence $y=1$ and $w^{\prime}=w$. This proves (a). Now (b) is already proved. Note that by (b), w has the property in (c). Conversely, let $w^{\prime} \in W_{I} a$ be an element such that $l\left(s w^{\prime}\right)>l\left(w^{\prime}\right)$ for all $s \in I$. We have $w^{\prime}=y w$ for some $y \in W_{I}$. If $y \neq 1$ then for some $s \in I$ we have $l(y)=l(s y)+1$. By (b) we have $l\left(w^{\prime}\right)=l(y)+l(w), l\left(s w^{\prime}\right)=l(s y)+l(w)$. Thus $l\left(w^{\prime}\right)-l\left(s w^{\prime}\right)=l(y)-l(s y)=1$, a contradiction. Thus $y=1$ and $w^{\prime}=w$. The lemma is proved.

Lemma 9.8. Let $W_{I}$ a be a coset in $W$.

(a) If $W_{I}$ is finite, this coset has a unique element $w$ of maximal length. If $W_{I}$ is infinite, this coset has no element of maximal length.

(b) Assume that $W_{I}$ is finite. If $y \in W_{I}$ then $l(y w)=l(w)-l(y)$.

(c) Assume that $W_{I}$ is finite. Then $w$ is characterized by the property that $l(s w)<l(w)$ for all $s \in I$.

Assume that $w$ has maximal length in $W_{I} a$. We show that for any $y \in W_{I}$ we have

(d) $l(y w)=l(w)-l(y)$.

We argue by induction on $l(y)$. If $l(y)=0$, the result is clear. Assume now that $l(y)=p+1 \geq 1$. Let $y=s_{1} \ldots s_{p} s_{p+1}$ be a reduced expression. By the induction hypothesis, $l(w)=l\left(s_{1} s_{2} \ldots s_{p} w\right)+p$. Hence we can find a reduced expression of $w$ of the form $s_{p} \ldots s_{2} s_{1} s_{1}^{\prime} s_{2}^{\prime} \ldots s_{q}^{\prime}$. Since $s_{p+1} \in I$, by our assumption on $w$ we have $l\left(s_{p+1} w\right)=l(w)-1$. Using 1.7, we deduce that either

(1) $s_{p+1} s_{p} \ldots s_{j+1}=s_{p} \ldots s_{j+1} s_{j}$ for some $j \in[1, p]$ or

(2) $s_{p+1} s_{p} \ldots s_{2} s_{1} s_{1}^{\prime} s_{2}^{\prime} \ldots s_{i+1}^{\prime}=s_{p} \ldots s_{2} s_{1} s_{1}^{\prime} s_{2}^{\prime} \ldots s_{i+1}^{\prime} s_{i}^{\prime}$ for some $i \in[1, q]$.

In case (1) it follows that $y=s_{1} \ldots s_{p} s_{p+1}=s_{1} s_{2} \ldots s_{j-1} s_{j+1} \ldots s_{p}$, contradicting $l(y)=p+1$. Thus, we must be in case (2). We have

$$
y w=s_{1}^{\prime} s_{2}^{\prime} \ldots s_{i-1}^{\prime} s_{i+1}^{\prime} \ldots s_{q}^{\prime} \text { and } l(y w) \leq q-1=l(w)-p-1=l(w)-l(y) .
$$

Thus, $l(w) \geq l(y w)+l(y)$. The reverse inequality is obvious. Hence $l(w)=$ $l(y w)+l(y)$. This completes the induction. 
From $(\mathrm{d})$ we see that $l(y) \leq l(w)$. Thus $l: W_{I} \rightarrow \mathbf{N}$ is bounded above. Hence there exists $y \in W_{I}$ of maximal length in $W_{I}$. Applying (d) to $y, W_{I}$ instead of $w, W_{I} a$ we see that

$$
l(y)=l\left(y^{\prime-1}\right)+l\left(y^{\prime-1} y\right)=l\left(y^{\prime}\right)+l\left(y^{\prime-1} y\right)
$$

for any $y^{\prime} \in W_{I}$. Hence a reduced expression of $y^{\prime}$ followed by a reduced expression of $y^{\prime-1} y$ gives a reduced expression of $y$. In particular $y^{\prime} \leq y$. Since the set $\left\{y^{\prime} \in W ; y^{\prime} \leq y\right\}$ is finite, we see that $W_{I}$ is finite. Conversely, if $W_{I}$ is finite then $W_{I} a$ clearly has some element of maximal length.

If $w^{\prime}$ is another element of maximal length in $W_{I} a$ then $w^{\prime}=y w$ for some $y \in W_{I}$. We have $l(w)=l\left(w^{\prime}\right)=l(w)-l(y)$ hence $l(y)=0$ hence $y=1$ and $w^{\prime}=w$. This proves (a) and (b). The proof of (c) is entirely similar to that of 9.7(c). The lemma is proved.

9.9. Replacing $W, L$ by $W_{I},\left.L\right|_{W_{I}}$ in the definition of $\mathcal{H}$ we obtain an $\mathcal{A}$-algebra $\mathcal{H}_{I}$ (naturally a subalgebra of $\mathcal{H}$ ); instead of $r_{x, y}, p_{x, y}, c_{y}, \mu_{x, y}^{s}$ we obtain for $x, y \in W_{I}$ elements $r_{x, y}^{I} \in \mathcal{A}, p_{x, y}^{I} \in \mathcal{A}_{\leq 0}, c_{y}^{I} \in \mathcal{H}_{I}, \mu_{x, y}^{s, I} \in \mathcal{A}$.

Lemma 9.10. Let $z \in W$ be such that $z$ is the element of minimal length of $W_{I} z$. Let $x, y \in W_{I}$. We have

(a) $\left\{u^{\prime} \in W ; x z \leq u^{\prime} \leq y z\right\}=\left\{u \in W_{I} ; x \leq u \leq y\right\} z$;

(b) $r_{x z, y z}=r_{x, y}^{I}$;

(c) $p_{x z, y z}=p_{x, y}^{I}$;

(d) $c_{y}^{I}=c_{y}$.

(e) If in addition, $s \in I$ and $s x<x<y<$ sy, then $s x z<x z<y z<$ syz and $\mu_{x, y}^{s, I}=\mu_{x z, y z}^{s}$.

We first prove the following statement.

Assume that $z_{1}, z_{2}$ have minimal length in $W_{I} z_{1}, W_{I} z_{2}$ respectively, that $u_{1}, u_{2} \in$ $W_{I}$ and that $u_{1} z_{1} \leq u_{2} z_{2}$. Then

(f) $z_{1} \leq z_{2}$; if in addition, $z_{1}=z_{2}$ then $u_{1} \leq u_{2}$.

Indeed, using 2.4 we see that there exist $u_{1}^{\prime}, z_{1}^{\prime}$ such that

$u_{1} z_{1}=u_{1}^{\prime} z_{1}^{\prime}, u_{1}^{\prime} \leq u_{2}, z_{1}^{\prime} \leq z_{2}$.

Then $u_{1}^{\prime} \in W_{I}$ and $z_{1}^{\prime} \in W_{I} z_{1}$ hence $z_{1}^{\prime}=w z_{1}$ where $w \in W_{I}, l\left(z_{1}^{\prime}\right)=l(w)+l\left(z_{1}\right)$. Hence $z_{1} \leq z_{1}^{\prime}$. Since $z_{1}^{\prime} \leq z_{2}$, we see that $z_{1} \leq z_{2}$. If we know that $z_{1}=z_{2}$, then $z_{1}^{\prime}=z_{1}$ hence $u_{1}=u_{1}^{\prime}$. Since $u_{1}^{\prime} \leq u_{2}$, it follows that $u_{1} \leq u_{2}$ and (f) is proved.

We prove (a). If $u \in W_{I}$ and $x \leq u \leq y$, then $x z \leq u z \leq y z$ by 2.4 and 9.7(b). Conversely, assume that $u^{\prime} \in W$ satisfies $x z \leq u^{\prime} \leq y z$. Then $u^{\prime}=u z_{1}$ where $z_{1}$ has minimal length in $W_{I} u^{\prime}$ and $u \in W_{I}$. Applying (f) to $x z \leq u z_{1}$ and to $u z_{1} \leq y z$ we deduce $z \leq z_{1} \leq z$. Hence $z=z_{1}$. Applying the second part of (f) to $x z \leq u z$ and to $u z \leq y z$ we deduce $x \leq u \leq y$. This proves (a).

We prove (b) by induction on $l(y)$. Assume first that $y=1$. Then $r_{x, y}^{I}=\delta_{x, 1}$. Now $r_{x z, z}=0$ unless $x z \leq z($ see $4.7(\mathrm{a}))$ in which case $x=1$ and $r_{z, z}=1$. Thus, 
(b) holds for $y=1$. Assume now that $l(y) \geq 1$. We can find $s \in I$ such that $l(s y)=l(y)-1$. We have

$$
l(s y z)=l(s y)+l(z)=l(y)-1+l(z)=l(y z)-1 .
$$

If $s x<x$ then we have (as above) $s x z<x z$. Using 4.4 and the induction hypothesis, we have

$$
r_{x z, y z}=r_{s x z, s y z}=r_{s x, s y}^{I}=r_{x, y}^{I} .
$$

If $s x>x$ then we have (as above) $s x z>x z$. Using 4.4 and the induction hypothesis, we have

$$
r_{x z, y z}=r_{s x z, s y z}+\left(v_{s}-v_{s}^{-1}\right) r_{x z, s y z}=r_{s x, s y}^{I}+\left(v_{s}-v_{s}^{-1}\right) r_{x, s y}^{I}=r_{x, y}^{I} .
$$

This completes the proof of (b).

We prove (c). Using (a), we may assume that $x \leq y$ (otherwise, both sides are zero.) We argue by induction on $l(y)-l(x) \geq 0$. If $y=x$, the result is clear (both sides are 1). Assume now that $l(y)-l(x) \geq 1$. Using 5.3, then (a),(b) and the induction hypothesis, we have

$$
\begin{gathered}
\bar{p}_{x z, y z}=\sum_{u^{\prime} ; x z \leq u^{\prime} \leq y z} r_{x z, u^{\prime}} p_{u^{\prime}, y z}=\sum_{u \in W_{I} ; x \leq u \leq y} r_{x z, u z} p_{u z, y z} \\
=\sum_{u \in W_{I} ; x \leq u \leq y} r_{x, u}^{I} p_{u z, y z}=\sum_{u \in W_{I} ; x<u \leq y} r_{x, u}^{I} p_{u, y}^{I}+p_{x z, y z} .
\end{gathered}
$$

Using 5.3 for $W_{I}$ we have $\bar{p}_{x, y}^{I}=\sum_{y ; x \leq u \leq y} r_{x, u}^{I} p_{u, y}^{I}$. Comparison with the previous equality gives

$$
\bar{p}_{x z, y z}-\bar{p}_{x, y}^{I}=p_{x z, y z}-p_{x, y}^{I} \text {. }
$$

The right hand side of this equality is in $\mathcal{A}_{<0}$. Since it is fixed by ${ }^{-}$, it must be 0 . This proves (c). Now (d) is an immediate consequence of (c) (with $z=1$ ).

We prove (e). By 6.3(ii),

$$
\sum_{u^{\prime} ; x z \leq u^{\prime}<y z ; s u^{\prime}<u^{\prime}} p_{x z, u^{\prime}} \mu_{u^{\prime}, y z}^{s}-v_{s} p_{x z, y z} \in \mathcal{A}_{<0} .
$$

We rewrite this using (a):

$$
\sum_{u \in W_{I} ; x \leq u<y ; s u<u} p_{x z, u z} \mu_{u z, y z}^{s}-v_{s} p_{x z, y z} \in \mathcal{A}_{<0} .
$$

We may assume that for all $u$ in the sum, other than $u=x$, we have $\mu_{u z, y z}^{s}=\mu_{u, y}^{s, I}$. Using this and (d), we obtain

$$
\mu_{x z, y z}^{s}+\sum_{u \in W_{I} ; x<u<y ; s u<u} p_{x, u}^{I} \mu_{u, y}^{s, I}-v_{s} p_{x, y}^{I} \in \mathcal{A}_{<0}
$$

By 6.3(ii) for $W_{I}$ we have

$$
\mu_{x, y}^{s, I}+\sum_{u \in W_{I} ; x<u<y ; s u<u} p_{x, u}^{I} \mu_{u, y}^{s, I}-v_{s} p_{x, y}^{I} \in \mathcal{A}_{<0} .
$$

It follows that $\mu_{x z, y z}^{s}-\mu_{x, y}^{s, I} \in \mathcal{A}_{<0}$. On the other hand, $\mu_{x z, y z}^{s}-\mu_{x, y}^{s, I}$ is fixed by ${ }^{-}$ (see 6.3(ii)) hence it is 0 . This proves (e). The lemma is proved. 
Proposition 9.11. Assume that $L(s)>0$ for all $s \in I$.

(a) Let $z \in W$ be such that $z$ is the element of minimal length of $W_{I} z$. If $x, y$ in $W_{I}$ satisfy $x \leq_{\mathcal{L}} y$ (relative to $W_{I}$ ), then $x z \leq_{\mathcal{L}} y z$ (in $W$ ). If $x, y$ in $W_{I}$ satisfy $x \sim_{\mathcal{L}} y$ (relative to $W_{I}$ ), then $x z \sim_{\mathcal{L}} y z$ (in $\left.W\right)$.

(b) Let $z \in W$ be such that $z$ is the element of minimal length of $z W_{I}$. If $x, y$ in $W_{I}$ satisfy $x \leq_{\mathcal{R}} y$ (relative to $W_{I}$ ), then $z x \leq_{\mathcal{R}} z y$ (in $W$ ). If $x, y$ in $W_{I}$ satisfy $x \sim_{\mathcal{R}} y$ (relative to $W_{I}$ ), then $z x \sim_{\mathcal{R}} z y($ in $W$ ).

We prove the first assertion of (a). We may assume that $x \leftarrow \mathcal{L} y$ (relative to $\left.W_{I}\right)$ and $x \neq y$. Thus, there exists $s \in I$ such that $s y>y, s x<x$ and we have either $x=s y$ or $x<y$ and $\mu_{x, y}^{s} \neq 0$. If $x=s y$, then $s x z<x z=s y z>y z$, hence $x z \leftarrow_{\mathcal{L}} y z$ (in $W$ ). Thus, we may assume that $x<y$ and $\mu_{x, y}^{s} \neq 0$. By 9.10(e) we then have $\mu_{x z, y z}^{s} \neq 0$, hence $x z \leftarrow \mathcal{L} y z$ (in $W$ ). The first assertion of (a) is proved. The second assertion of (a) follows from the first. (b) follows by applying (a) to $z^{-1}, x^{-1}, y^{-1}$ instead of $z, x, y$.

9.12. Assume that $z \in W$ is such that $W_{I} z=z W_{I}$ and $z$ is the element of minimal length of $W_{I} z=z W_{I}$. Then $y \mapsto z^{-1} y z$ is an automorphism of $W_{I}$. If $s \in I$ then, by 9.7 , we have $l(s z)=l(s)+l(z)=1+l(z)$; by 9.7 applied to $W_{I} z^{-1}$ instead of $W_{I} z$ we have $l\left(\left(z^{-1} s z\right) z^{-1}\right)=l\left(z^{-1} s z\right)+l\left(z^{-1}\right)$ hence $l\left(z^{-1} s\right)=l\left(z^{-1} s z\right)+l\left(z^{-1}\right)$; since $l\left(z^{-1} s\right)=l(s z)$ and $l\left(z^{-1}\right)=l(z)$, it follows that $l\left(z^{-1} s z\right)+l\left(z^{-1}\right)=1+l(z)$, hence $l\left(z^{-1} s z\right)=1$. We see that $y \mapsto z^{-1} y z$ maps $I$ onto itself hence it is an automorphism of $W_{I}$ as a Coxeter group. This automorphism preserves the function $\left.L\right|_{W_{I}}$. Indeed, if $y \in W_{I}$, then

$l\left(z y z^{-1}\right)+l(z)=l\left(\left(z y z^{-1}\right) z\right)=l(z y)=l\left(y^{-1} z^{-1}\right)=l\left(y^{-1}\right)+l\left(z^{-1}\right)=l(y)+l(z)$

(by 9.7 applied to $W_{I} z$ and to $W_{I} z^{-1}$ ) hence

$$
\begin{aligned}
& L\left(z y z^{-1}\right)+L(z)=L\left(\left(z y z^{-1}\right) z\right)=L(z y)=L\left(y^{-1} z^{-1}\right)=L\left(y^{-1}\right)+L\left(z^{-1}\right) \\
& =L(y)+L(z)
\end{aligned}
$$

so that $L\left(z y z^{-1}\right)=L(z)$. In particular, this automorphism respects the preorders $\leq_{\mathcal{L}}, \leq_{\mathcal{R}}, \leq_{\mathcal{L} \mathcal{R}}$ of $W_{I}$ (defined in terms of $\left.L\right|_{W_{I}}$ ) and the associated equivalence relations.

Proposition 9.13. Assume that $L(s)>0$ for all $s \in I$. Let $z$ be as in 9.12. If $x, y$ in $W_{I}$ satisfy $x \leq_{\mathcal{L R}} y$ (relative to $W_{I}$ ), then $x z \leq_{\mathcal{L R}} y z$ (in $W$ ). If $x, y$ in $W_{I}$ satisfy $x \sim_{\mathcal{L R}} y$ (relative to $W_{I}$ ), then $x z \sim_{\mathcal{L R}} y z($ in $W)$.

We prove the first assertion. We may assume that either $x \leq_{\mathcal{L}} y$ (in $W_{I}$ ) or $x \leq_{\mathcal{R}} y$ (in $\left.W_{I}\right)$. In the first case, by $9.11(\mathrm{a})$, we have $x z \leq_{\mathcal{L}} y z$ (in $W$ ) hence $x z \leq_{\mathcal{L R}} y z$ (in $W$ ). In the second case, by 9.12 , we have $z^{-1} x z \leq_{\mathcal{R}} z^{-1} y z$. Applying 9.11(b) to $z^{-1} x z, z^{-1} y z$ instead of $x, y$ we see that $x z \leq_{\mathcal{R}} y z$ (in $W$ ) hence $x z \leq_{\mathcal{L R}} y z$ (in $W$ ). This proves the first assertion. The second assertion follows from the first. 


\section{INVERSION}

10.1. We preserve the setup of 3.1. For $y, w \in W$ we set

$$
q_{y, w}^{\prime}=\sum(-1)^{n} p_{z_{0}, z_{1}} p_{z_{1}, z_{2}} \ldots p_{z_{n-1}, z_{n}} \in \mathcal{A}
$$

(sum over all sequences $y=z_{0}<z_{1}<z_{2}<\cdots<z_{n}=w$ in $W$ ) and

$$
q_{y, w}=\operatorname{sgn}(y) \operatorname{sgn}(w) q_{y, w}^{\prime} .
$$

We have

$$
\begin{aligned}
& q_{w, w}=1, \\
& q_{y, w} \in \mathcal{A}_{<0} \text { if } y \neq w, \\
& q_{y, w}=0 \text { unless } y \leq w .
\end{aligned}
$$

Proposition 10.2. For any $y, w \in W$ we have $\bar{q}_{y, w}=\sum_{z ; y \leq z \leq w} q_{y, z} r_{z, w}$.

The (triangular) matrices $Q^{\prime}=\left(q_{y, w}^{\prime}\right), P=\left(p_{y, w}\right), R=\left(r_{y, w}\right)$ are related by

(a) $Q^{\prime} P=P Q^{\prime}=1, \bar{P}=R P, \bar{R} R=R \bar{R}=1$

where ${ }^{-}$over a matrix is the matrix obtained by applying ${ }^{-}$to each entry. (Although the matrices may be infinite, the products are well defined as each entry of a product is obtained by finitely many operations.) The last three equations in (a) are obtained from 5.3, 4.6; the equations involving $Q^{\prime}$ follow from the definition. From (a) we deduce $Q^{\prime} P=1=\bar{Q}^{\prime} \bar{P}=\bar{Q}^{\prime} R P$. Hence $Q^{\prime} P=\bar{Q}^{\prime} R P$. Multiplying on the right by $Q^{\prime}$ and using $P Q^{\prime}=1$ we deduce $Q^{\prime}=\bar{Q}^{\prime} R$. Multiplying on the right by $\bar{R}$ gives

(b) $\bar{Q}^{\prime}=Q^{\prime} \bar{R}$.

Let $\mathbf{s}$ be the matrix whose $y, w$ entry is $\operatorname{sgn}(y) \delta_{y, w}$. We have $\mathbf{s}^{2}=1$. Let $Q$ be the triangular matrix $\left(q_{y, w}\right)$. Note that $Q=\mathbf{s} Q^{\prime} \mathbf{s}$. By 4.5 we have $\bar{R}=\mathbf{s} R \mathbf{s}$. Hence by multiplying the two sides of (b) on the left and right by $\mathbf{s}$ we obtain $\bar{Q}=Q R$. The proposition is proved.

10.3. Define an $\mathcal{A}$-linear map $\tau: \mathcal{H} \rightarrow \mathcal{A}$ by $\tau\left(T_{w}\right)=\delta_{w, 1}$ for $w \in W$.

Lemma 10.4. (a) For $x, y \in W$ we have $\tau\left(T_{x} T_{y}\right)=\delta_{x y, 1}$.

(b) For $h, h^{\prime} \in \mathcal{H}$ we have $\tau\left(h h^{\prime}\right)=\tau\left(h^{\prime} h\right)$.

(c) Let $x, y, z \in W$ and let $M=\min (L(x), L(y), L(z))$. We have $\tau\left(T_{x} T_{y} T_{z}\right) \in$ $v^{M} \mathbf{Z}\left[v^{-1}\right]$.

We prove (a) by induction on $l(y)$. If $l(y)=0$, the result is clear. Assume now that $l(y) \geq 1$. If $l(x y)=l(x)+l(y)$ then $T_{x} T_{y}=T_{x y}$ and the result is clear. Hence we may assume that $l(x y) \neq l(x)+l(y)$. Then $l(x y)<l(x)+l(y)$. Let $y=s_{1} s_{2} \ldots s_{q}$ be a reduced expression. We can find $i \in[1, q]$ such that

$$
l(x)+i-1=l\left(x s_{1} s_{2} \ldots s_{i-1}\right)>l\left(x s_{1} s_{2} \ldots s_{i-1} s_{i}\right) .
$$


We show that

$$
x s_{1} s_{2} \ldots s_{i-1} s_{i+1} \ldots s_{q} \neq 1 \text {. }
$$

If (e) does not hold, then $x=s_{q} \ldots s_{i+1} s_{i-1} \ldots s_{1}$, so that

$$
\begin{gathered}
l\left(x s_{1} s_{2} \ldots s_{i-1}\right)=l\left(s_{q} \ldots s_{i+1} s_{i-1} \ldots s_{1} s_{1} \ldots s_{i-1}\right)=l\left(s_{q} \ldots s_{i+1}\right)=q-i \\
l\left(x s_{1} s_{2} \ldots s_{i-1} s_{i}\right)=l\left(s_{q} \ldots s_{i+1} s_{i-1} \ldots s_{1} s_{1} \ldots s_{i}\right)=l\left(s_{q} \ldots s_{i+1} s_{i}\right)=q-i+1
\end{gathered}
$$

contradicting (d). Thus (e) holds. We have

$$
\begin{aligned}
& \tau\left(T_{x} T_{y}\right)=\tau\left(T_{x} T_{s_{1}} T_{s_{2}} \ldots T_{s_{q}}\right)=\tau\left(T_{x s_{1} s_{2} \ldots s_{i-1}} T_{s_{i}} T_{s_{i+1} \ldots s_{q}}\right) \\
& =\tau\left(T_{x s_{1} s_{2} \ldots s_{i-1} s_{i}} T_{s_{i+1} \ldots s_{q}}\right)+\left(v_{s}-v_{s}^{-1}\right) \tau\left(T_{x s_{1} s_{2} \ldots s_{i-1}} T_{s_{i+1} \ldots s_{q}}\right) .
\end{aligned}
$$

By the induction hypothesis and (d), this equals

$$
\delta_{x s_{1} s_{2} \ldots s_{i-1} s_{i} s_{i+1} \ldots s_{q}, 1}+\left(v_{s}-v_{s}^{-1}\right) \delta_{x s_{1} s_{2} \ldots s_{i-1} s_{i+1} \ldots s_{q}, 1}=\delta_{x y, 1}
$$

This completes the proof of (a). To prove (b), we may assume that $h=T_{x}, h^{\prime}=T_{y}$ for $x, y \in W$; we then use (a) and the obvious equality $\delta_{x y, 1}=\delta_{y x, 1}$.

We prove (c). Using (b) we see that $\tau\left(T_{x} T_{y} T_{z}\right)=\tau\left(T_{y} T_{z} T_{x}\right)=\tau\left(T_{z} T_{x} T_{y}\right)$. Hence it is enough to show that, for any $x, y, z$ we have

$$
\tau\left(T_{x} T_{y} T_{z}\right) \in v^{L(x)} \mathbf{Z}\left[v^{-1}\right]
$$

We argue by induction on $l(x)$. If $l(x)=0$, then $x=1$ and the result follows from (a). Assume now that $l(x) \geq 1$. We can find $s \in S$ such that $x s<x$. If $s y>y$, then by the induction hypothesis,

$$
\tau\left(T_{x} T_{y} T_{z}\right)=\tau\left(T_{x s} T_{s y} T_{z}\right) \in v^{L(x)-L(s)} \mathbf{Z}\left[v^{-1}\right] \subset v^{L(x)} \mathbf{Z}\left[v^{-1}\right]
$$

If $s y<y$, then by the induction hypothesis,

$$
\begin{aligned}
& \tau\left(T_{x} T_{y} T_{z}\right)=\tau\left(T_{x s} T_{s y} T_{z}\right)+\left(v_{s}-v_{s}^{-1}\right) \tau\left(T_{x s} T_{y} T_{z}\right) \\
& \in v^{L(x)-L(s)} \mathbf{Z}\left[v^{-1}\right]+v_{s} v^{L(x)-L(s)} \mathbf{Z}\left[v^{-1}\right] \subset v^{L(x)} \mathbf{Z}\left[v^{-1}\right] .
\end{aligned}
$$

The lemma is proved.

10.5. Let $\mathcal{H}^{\prime}=\operatorname{Hom}_{\mathcal{A}}(\mathcal{H}, \mathcal{A})$. We regard $\mathcal{H}^{\prime}$ as a left $\mathcal{H}$-module where, for $h \in$ $\mathcal{H}, \phi \in \mathcal{H}^{\prime}$ we have $(h \phi)\left(h_{1}\right)=\phi\left(h_{1} h\right)$ for all $h_{1} \in \mathcal{H}$ and as a right $\mathcal{H}$-module where, for $h \in \mathcal{H}, \phi \in \mathcal{H}^{\prime}$, we have $(\phi h)\left(h_{1}\right)=\phi\left(h h_{1}\right)$ for all $h_{1} \in \mathcal{H}$. 
10.6. We sometimes identify $\mathcal{H}^{\prime}$ with the set of all formal sums $\sum_{x \in W} a_{x} T_{x}$ with $a_{x} \in \mathcal{A}$; to $\phi \in \mathcal{H}^{\prime}$ corresponds the formal sum $\sum_{x \in W} \phi\left(T_{x^{-1}}\right) T_{x}$. Since $\mathcal{H}$ is contained in the set of such formal sums (it is the set of sums such that $c_{x}=0$ for all but finitely many $x$ ), we see that $\mathcal{H}$ is naturally a subset of $\mathcal{H}^{\prime}$. Using 10.4(a) we see that the imbedding $\mathcal{H} \subset \mathcal{H}^{\prime}$ is an imbedding of $\mathcal{H}$-bimodules; it is an equality if $W$ is finite.

10.7. Let $z \in W$. Recall that in 8.1 we have defined $D_{z} \in \mathcal{H}^{\prime}$ by $D_{z}\left(c_{w}\right)=\delta_{z, w}$ for all $w$. An equivalent definition is

(a) $D_{z}\left(T_{y}\right)=q_{z, y}^{\prime}$

for all $y \in W$. Indeed, assuming that (a) holds, we have

$$
D_{z}\left(c_{w}\right)=\sum_{y} q_{z, y}^{\prime} p_{y, w}=\delta_{z, w}
$$

Proposition 10.8. Let $z \in W, s \in S$. Assume that $L(s)>0$.

(a) If zs $<z$, then $c_{s} D_{z}=\left(v_{s}+v_{s}^{-1}\right) D_{z}+D_{z s}+\sum_{u ; z<u<u s} \mu_{z^{-1}, u^{-1}} D_{u}$.

(b) If $z s>z$, then $c_{s} D_{z}=0$.

For $a, b \in W$ we define $\delta_{a<b}$ to be 1 if $a<b$ and 0 otherwise. Let $w \in W$. If $w s>w$, then by $6.7(\mathrm{a})$, we have

$$
\begin{aligned}
& \left(c_{s} D_{z}\right)\left(c_{w}\right)=D_{z}\left(c_{w} c_{s}\right)=D_{z}\left(c_{w s}+\sum_{\substack{x \\
x s<x<w}} \mu_{x^{-1}, w^{-1}}^{s} c_{x}\right) \\
& =\delta_{z, w s}+\sum_{\substack{x \\
x s<x<w}} \mu_{x^{-1}, w^{-1}}^{s} \delta_{z, x} .
\end{aligned}
$$

If $w s<w$, then by $6.7(\mathrm{~b})$, we have

$$
\left(c_{s} D_{z}\right)\left(c_{w}\right)=D_{z}\left(c_{w} c_{s}\right)=\left(v_{s}+v_{s}^{-1}\right) D_{z}\left(c_{w}\right)=\left(v_{s}+v_{s}^{-1}\right) \delta_{z, w} .
$$

If $z s<z, w s>w$, then by (c):

$$
\left.\left(c_{s} D_{z}\right)\left(c_{w}\right)=\delta_{z s, w}+\delta_{z<w} \mu_{z^{-1}, w^{-1}}^{s}=\left(v_{s}+v_{s}^{-1}\right) D_{z}+D_{z s}+\sum_{\substack{u \\ z<u<u s}} \mu_{z^{-1}, u^{-1}}^{s} D_{u}\right)\left(c_{w}\right)
$$

If $z s<z, w s<w$, then by $(\mathrm{d})$ :

$$
\left.\left(c_{s} D_{z}\right)\left(c_{w}\right)=\left(v_{s}+v_{s}^{-1}\right) \delta_{z, w}=\left(v_{s}+v_{s}^{-1}\right) D_{z}+D_{z s}+\sum_{\substack{u \\ z<u<u s}} \mu_{z^{-1}, u^{-1}}^{s} D_{u}\right)\left(c_{w}\right)
$$

If $z s>z, w s>w$, then by (c), we have $\left(c_{s} D_{z}\right)\left(c_{w}\right)=0$. If $z s>z, w s<w$, then by $(\mathrm{d})$, we have $\left(c_{s} D_{z}\right)\left(c_{w}\right)=0$. Since $\left(c_{w}\right)$ is an $\mathcal{A}$-basis of $\mathcal{H}$, the proposition follows. 
10.9. We show that

$$
\left\{ \pm c_{w} ; w \in W\right\}=\left\{h \in \mathcal{H} ; \tau\left(h h^{b}\right) \in 1+\mathcal{A}_{<0} ; \bar{h}=h\right\} .
$$

For any $w, w^{\prime} \in W$ we have

$$
\tau\left(c_{w} c_{w^{\prime}}^{b}\right)=\tau\left(\sum_{y, y^{\prime}} p_{y, w} p_{y^{\prime}, w^{\prime}} T_{y} T_{y^{\prime}-1}\right)=\sum_{y, y^{\prime}} p_{y, w} p_{y^{\prime}, w^{\prime}} \delta_{y, y^{\prime}}=\delta_{w, w^{\prime}}+z_{w, w^{\prime}}
$$

where $z_{w, w^{\prime}} \in \mathcal{A}_{<0}$. In particular, $\tau\left(c_{w} c_{w}^{b}\right) \in 1+\mathcal{A}_{<0}$.

Conversely, assume that $h \in \mathcal{H}$ satisfies $\tau\left(h h^{b}\right) \in 1+\mathcal{A}_{<0}, \bar{h}=h$. We have $h=\sum_{w \in W} x_{w} c_{w}$ where $x_{w} \in \mathcal{A}$ are 0 for all but finitely many $w$. We can find $t \in \mathbf{Z}$ such that $x_{w}=b_{w} v^{t} \bmod \mathcal{A}_{<t}$ where $b_{w} \in \mathbf{Z}$ for all $w$ and $b_{w} \neq 0$ for some $w$. Using (b), we have

$$
\begin{aligned}
& \tau\left(h h^{b}\right)=\tau\left(\sum_{w, w^{\prime}} x_{w} x_{w^{\prime}} c_{w} c_{w^{\prime}}^{b}\right)=\sum_{w, w^{\prime}} x_{w} x_{w^{\prime}}\left(\delta_{w, w^{\prime}}+z_{w, w^{\prime}}\right) \\
& =\sum_{w} x_{w}^{2}+\sum_{w \neq w^{\prime}} x_{w} x_{w^{\prime}} z_{w, w^{\prime}} .
\end{aligned}
$$

This equals $\sum_{w} b_{w}^{2} v^{2 t}$ modulo $\mathcal{A}_{<2 t}$ and also equals 1 modulo $\mathcal{A}_{<0}$. It follows that $t=0$ and $\sum_{w} b_{w}^{2}=1$. Since $b_{w}$ are integers, there exists $u \in W$ such that $b_{u}= \pm 1$ and $b_{w}=0$ for $w \neq u$. Thus $x_{w} \in \mathcal{A}_{\leq 0}$ for all $w$. Since $\bar{h}=h$ we have $\bar{x}_{w}=x_{w}$ for all $w$. It follows that $x_{w}=b_{w}$ for all $w$. Thus $h= \pm c_{u}$. This proves (a).

10.10. The interest of $10.9(\mathrm{a})$ is that it provides a definition of $c_{w}$ (up to sign) without using the basis $T_{w}$ of $\mathcal{H}$ (instead, it uses $\tau: \mathcal{H} \rightarrow \mathcal{A}$ ). The equality 10.9(a) could be used to give a definition of $c_{w}$ (up to sign) in more general situations than that considered above, when the basis $\left(T_{w}\right)$ is not defined but $\tau: \mathcal{H} \rightarrow \mathcal{A}$ is defined (it is known that $\tau$ is defined when $W$ is replaced by certain complex reflection groups, see $[\mathrm{BM}])$. This should lead to a definition of cells for complex reflection groups.

\section{The LOngest element For A Finite $W$}

11.1. We preserve the setup of 3.1. Let $I \subset S$ be such that $W_{I}$ is finite. By 9.8 , there is a unique element of maximal length of $W_{I}$. We denote it by $w_{0}^{I}$. If $w_{1}$ has minimal length in $W_{I} a$ then $w_{0}^{I} w_{1}$ has maximal length in $W_{I} a$.

11.2. In the remainder of this section we assume that $W$ is finite. Then $w_{0}:=w_{0}^{S}$, the unique element of maximal length of $W$, is well defined. Since $l\left(w_{0}^{-1}\right)=l\left(w_{0}\right)$, we must have $w_{0}^{-1}=w_{0}$. By the argument in the proof of 9.8 we have $w \leq w_{0}$ for any $w \in W$. By 9.8 we have

(a) $l\left(w w_{0}\right)=l\left(w_{0}\right)-l(w)$

for any $w \in W$. Applying this to $w^{-1}$ and using the equalities $l\left(w^{-1} w_{0}\right)=$ $l\left(w_{0}^{-1} w\right)=l\left(w_{0} w\right), l\left(w^{-1}\right)=l(w)$, we deduce that 
(b) $l\left(w_{0} w\right)=l\left(w_{0}\right)-l(w)$.

We can rewrite $(\mathrm{a}),(\mathrm{b})$ as $l\left(w_{0}\right)=l\left(w^{-1}\right)+l\left(w w_{0}\right), l\left(w_{0}\right)=l\left(w_{0} w\right)+l\left(w^{-1}\right)$. Using this and the definition of $L$ we deduce that

$$
L\left(w^{-1}\right)+L\left(w w_{0}\right)=L\left(w_{0}\right)=L\left(w_{0} w\right)+L\left(w^{-1}\right)
$$

hence $L\left(w w_{0}\right)=L\left(w_{0} w\right)$. This implies $L\left(w_{0} w w_{0}\right)=L(w)$ for all $w$. Replacing $L$ by $l$ gives $l\left(w_{0} w w_{0}\right)=l(w)$. Thus, the involution $w \mapsto w_{0} w w_{0}$ of $W$ maps $S$ into itself hence is a Coxeter group automorphism preserving the function $L$.

Lemma 11.3. Let $y, w \in W$. We have

(a) $y \leq w \Leftrightarrow w_{0} w \leq w_{0} y \Leftrightarrow w w_{0} \leq y w_{0}$;

(b) $r_{y, w}=r_{w w_{0}, y w_{0}}=r_{w_{0} w, w_{0} y}$;

(c) $\bar{p}_{w w_{0}, y w_{0}}=\sum_{z ; y \leq z \leq w} p_{z w_{0}, y w_{0}} r_{z, w}$.

We prove (a). To prove that $y \leq w \Longrightarrow w_{0} w \leq w_{0} y$, we may assume that $l(w)-l(y)=1, y w^{-1} \in T$. Then

$$
l\left(y w_{0}\right)-l\left(w w_{0}\right)=l\left(w_{0}\right)-l(y)-\left(l\left(w_{0}\right)-l(w)\right)=l(w)-l(y)=1
$$

and $\left(w w_{0}\right)\left(y w_{0}\right)^{-1}=w y^{-1} \in T$. Hence $w_{0} w \leq w_{0} y$. The opposite implication is proved in the same way. The second equivalence in (a) follows from the last sentence in 11.2.

We prove the first equality in (b) by induction on $l(w)$. If $l(w)=0$ then $w=1$. We have $r_{y, 1}=\delta_{y, 1}$. Now $r_{w_{0}, w_{0} y}$ is zero unless $w_{0} \leq w_{0} y$ (see 4.7). On the other hand we have $w_{0} y \leq w_{0}$ (see 11.2). Hence $r_{w_{0}, w_{0} y}$ is zero unless $w_{0} y=w_{0}$, that is unless $y=1$ in which case it is 1 . Thus the desired equality holds when $l(w)=0$. Assume now that $l(w) \geq 1$. We can find $s \in S$ such that $s w<w$. Then $s w w_{0}>w w_{0}$ by $(\mathrm{a})$.

Assume first that $s y<y$ (hence $\left.s y w_{0}>y w_{0}.\right)$ By 4.4 and the induction hypothesis we have

$$
r_{y, w}=r_{s y, s w}=r_{s w w_{0}, s y w_{0}}=r_{w w_{0}, y w_{0}}
$$

Assume next that $s y>y$ (hence $s y w_{0}<y w_{0}$. ) By 4.4 and the induction hypothesis we have

$$
\begin{aligned}
& r_{y, w}=r_{s y, s w}+\left(v_{s}-v_{s}^{-1}\right) r_{y, s w}=r_{s w w_{0}, s y w_{0}}+\left(v_{s}-v_{s}^{-1}\right) r_{s w w_{0}, y w_{0}} \\
& =r_{s w w_{0}, s y w_{0}}+\left(v_{s}-v_{s}^{-1}\right) r_{w w_{0}, s y w_{0}}=r_{w w_{0}, y w_{0}} .
\end{aligned}
$$

This proves the first equality in (b). The second equality in (b) follows from the last sentence in 11.2 .

We prove (c). We may assume that $y \leq w$. By 5.3 (for $w w_{0}, y w_{0}$ instead of $y, w)$ we have $\bar{p}_{w w_{0}, y w_{0}}=\sum_{z ; y \leq z \leq w} r_{w w_{0}, z w_{0}} p_{z w_{0}, y w_{0}}$ (we have used (a)). Here we substitute $r_{w w_{0}, z w_{0}}=r_{z, w}$ (see (b)) and the result follows. The lemma is proved. 
Proposition 11.4. For any $y, w \in W$ we have $q_{y, w}=p_{w w_{0}, y w_{0}}=p_{w_{0} w, w_{0} y}$.

The second equality follows from the last sentence in 11.2. We prove the first equality. We may assume that $y \leq w$. We argue by induction on $l(w)-l(y) \geq$ 0 . If $l(w)-l(y)=0$ we have $y=w$ and both sides are 1. Assume now that $l(w)-l(y) \geq 1$. Substracting the identity in $11.3(\mathrm{c})$ from that in 10.2 and using the induction hypothesis, we obtain

$$
\bar{q}_{y, w}-\bar{p}_{w w_{0}, y w_{0}}=q_{y, w}-p_{w w_{0}, y w_{0}} .
$$

The right hand side is in $\mathcal{A}_{<0}$; since it is fixed by ${ }^{-}$, it is 0 . The proposition is proved.

Proposition 11.5. We identify $\mathcal{H}=\mathcal{H}^{\prime}$ as in 10.6. If $z \in W$, then $D_{z^{-1}} \in \mathcal{H}^{\prime}$ (see 10.7) becomes an element of $\mathcal{H}$. We have $D_{z^{-1}} T_{w_{0}}^{-1}=\operatorname{sgn}\left(z w_{0}\right) c_{z w_{0}}^{\dagger},^{\dagger}$ as in 3.5 .

By definition, $D_{z^{-1}} \in \mathcal{H}$ is characterized by

$$
\tau\left(D_{z^{-1}} T_{y^{-1}}\right)=q_{z^{-1}, y^{-1}}^{\prime}
$$

for all $y \in W$. Here $\tau$ is as in 10.3. Hence, by $10.4(\mathrm{a})$, we have $D_{z^{-1}}=$ $\sum_{y} q_{z^{-1}, y^{-1}}^{\prime} T_{y}$. Using 11.4 , we deduce

$$
D_{z^{-1}}=\sum_{y} \operatorname{sgn}(y z) p_{w_{0} y^{-1}, w_{0} z^{-1}} T_{y}
$$

Multiplying on the right by $T_{w_{0}}^{-1}$ gives

$$
D_{z^{-1}} T_{w_{0}}^{-1}=\sum_{y} \operatorname{sgn}(y z) p_{w_{0} y^{-1}, w_{0} z^{-1}} T_{w_{0} y^{-1}}^{-1}
$$

since $T_{w_{0} y^{-1}} T_{y}=T_{w_{0}}$. On the other hand,

$$
\operatorname{sgn}\left(z w_{0}\right) c_{z w_{0}}^{\dagger}=\sum_{x} \operatorname{sgn}\left(z w_{0} x\right) p_{x, z w_{0}} T_{x^{-1}}^{-1}=\sum_{y} \operatorname{sgn}\left(z w_{0} y w_{0}\right) p_{y w_{0}, z w_{0}} T_{w_{0} y^{-1}}^{-1}
$$

We now use the identity $p_{y w_{0}, z w_{0}}=p_{w_{0} y^{-1}, w_{0} z^{-1}}$. The proposition follows.

Proposition 11.6. Let $u, z \in W, s \in S$ be such that $s z<z<u<s u$. Assume that $L(s)>0$. Then suw $w_{0}<u w_{0}<z w_{0}<s z w_{0}$ and $\mu_{u w_{0}, z w_{0}}^{s}=-\operatorname{sgn}(u z) \mu_{z, u}^{s}$.

Let $z \in W, s \in S$ be such that $s z<z$. Using $10.8(\mathrm{a})$, we see that

$$
\left(c_{s}-\left(v_{s}+v_{s}^{-1}\right)\right) D_{z^{-1}} T_{w_{0}}^{-1}=D_{z^{-1} s} T_{w_{0}}^{-1}+\sum_{u ; z^{-1}<u^{-1}<u^{-1} s} \mu_{z, u}^{s} D_{u^{-1}} T_{w_{0}}^{-1}
$$


hence, using 11.5, we have

$$
\left(c_{s}-\left(v_{s}+v_{s}^{-1}\right)\right) \operatorname{sgn}\left(z w_{0}\right) c_{z w_{0}}^{\dagger}=\operatorname{sgn}\left(z s w_{0}\right) c_{s z w_{0}}^{\dagger}+\sum_{u ; z<u<s u} \mu_{z, u}^{s} \operatorname{sgn}\left(u w_{0}\right) c_{u w_{0}}^{\dagger} .
$$

Applying ${ }^{\dagger}$ to both sides and using $\left(c_{s}-\left(v_{s}+v_{s}^{-1}\right)\right)^{\dagger}=-c_{s}$ gives

$$
-c_{s} c_{z w_{0}}=-c_{s z w_{0}}+\sum_{u ; z<u<s u} \mu_{z, u}^{s} \operatorname{sgn}(u z) c_{u w_{0}}
$$

Since $s z w_{0}>z w_{0}$, we can apply $6.6($ a) and we get

$$
c_{s} c_{z w_{0}}=c_{s z w_{0}}+\sum_{u^{\prime} ; s u^{\prime}<u^{\prime}<z w_{0}} \mu_{u^{\prime}, z w_{0}}^{s} c_{u^{\prime}}
$$

or equivalently

$$
c_{s} c_{z w_{0}}=c_{s z w_{0}}+\sum_{u ; z<u<s u} \mu_{u w_{0}, z w_{0}}^{s} c_{u w_{0}}
$$

Comparison with (a) gives

$$
-\sum_{u ; z<u<s u} \mu_{z, u}^{s} \operatorname{sgn}(u z) c_{u w_{0}}=\sum_{u ; z<u<s u} \mu_{u w_{0}, z w_{0}}^{s} c_{u w_{0}}
$$

the proposition follows.

Corollary 11.7. Assume that $L(s)>0$ for all $s \in S$. Let $y, w \in W$.

(a) $y \leq_{\mathcal{L}} w \Leftrightarrow w w_{0} \leq_{\mathcal{L}} y w_{0} \Leftrightarrow w_{0} w \leq_{\mathcal{L}} w_{0} y$;

(b) $y \leq_{\mathcal{R}} w \Leftrightarrow w w_{0} \leq_{\mathcal{R}} y w_{0} \Leftrightarrow w_{0} w \leq_{\mathcal{R}} w_{0} y$;

(c) $y \leq_{\mathcal{L R}} w \Leftrightarrow w w_{0} \leq_{\mathcal{L R}} y w_{0} \Leftrightarrow w_{0} w \leq_{\mathcal{L R}} w_{0} y$.

(d) Left multiplication by $w_{0}$ carries left cells to left cells, right cells to right cells, two-sided cells to two-sided cells. The same holds for right multiplication by $w_{0}$.

We prove the first equivalence in (a). It is enough to show that $y \leq_{\mathcal{L}} w \Longrightarrow$ $w w_{0} \leq_{\mathcal{L}} y w_{0}$. We may assume that $y \leftarrow_{\mathcal{L}} w$ and $y \neq w$. Then there exists $s \in S$ such that $s w>w, s y<y$ and $D_{y}\left(c_{s} c_{w}\right) \neq 0$. We have $s y w_{0}>y w_{0}, s w w_{0}<w w_{0}$. From 6.6 we see that either $y=w s$ or $y<w$ and $\mu_{y, w}^{s} \neq 0$. In the first case we have $w w_{0}=s y w_{0}$; in the second case we have $w w_{0}<y w_{0}$ and $\mu_{w w_{0}, y w_{0}}^{s} \neq 0$ (see 11.6). In both cases, 6.6 shows that $D_{w w_{0}}\left(c_{s} c_{y w_{0}}\right) \neq 0$. Hence $w w_{0} \leq_{\mathcal{L}} y w_{0}$. Thus, the first equivalence in (a) is established. The second equivalence in (a) follows from the last sentence in 11.2.

Now (b) follows by applying (a) to $y^{-1}, w^{-1}$ instead of (a); (c) follows from (a) and (b); (d) follows from (a),(b),(c). The corollary is proved. 


\section{Examples of Elements $D_{w}$}

We preserve the setup of 3.1.

Proposition 12.1. Assume that $L(s)>0$ for all $s \in S$. For any $y \in W$ we have $D_{1}\left(T_{y}\right)=\operatorname{sgn}(y) v^{-L(y)}$. Equivalently, with the identification in 10.6, we have $D_{1}=\sum_{y \in W} \operatorname{sgn}(y) v^{-L(y)} T_{y}$.

An equivalent statement is that $q_{1, y}^{\prime}=\operatorname{sgn}(y) v^{-L(y)}$. Since $q_{1, y}^{\prime}$ are determined by the equations $\sum_{y} q_{1, y}^{\prime} p_{y, w}=\delta_{1, w}$ (see 10.2(a)) it is enough to show that

$$
\sum_{y} \operatorname{sgn}(y) v^{-L(y)} p_{y, w}=\delta_{1, w}
$$

for all $w \in W$. If $w=1$ this is clear. Assume now that $w \neq 1$. We can find $s \in S$ such that $s w<w$. We must prove that

$$
\sum_{y ; y<s y} \operatorname{sgn}(y) v^{-L(y)}\left(p_{y, w}-v_{s}^{-1} p_{s y, w}\right)=0
$$

Each term of the last sum is 0, by 6.6(c). The proposition is proved.

Corollary 12.2. Assume that $W$ is finite and that $L(s)>0$ for all $s \in S$. Then $c_{w_{0}}=\sum_{y \in W} v^{-L\left(y w_{0}\right)} T_{y}$.

This follows immediately from 12.1 and 11.5. Alternatively, we can argue as follows. We prove that $p_{y, w_{0}}=v^{-L\left(y w_{0}\right)}$ for all $y$, by descending induction on $l(y)$. If $l(y)$ is maximal, that is $y=w_{0}$, then $p_{y, w_{0}}=1$. Assume now that $l(y)<l\left(w_{0}\right)$. We can find $s \in S$ such that $l(s y)=l(y)+1$. By the induction hypothesis we have $p_{s y, w_{0}}=v^{-L\left(s y w_{0}\right)}$. By $6.6(\mathrm{c})$, we have

$$
\begin{aligned}
& p_{y, w_{0}} \\
& =v_{s}^{-1} p_{s y, w_{0}}=v^{-L(s)-L\left(s y w_{0}\right)}=v^{-L(s)-L\left(w_{0}\right)+L(s y)}=v^{-L\left(w_{0}\right)+L(y)}=v^{-L\left(y w_{0}\right)} .
\end{aligned}
$$

The corollary is proved.

12.3. From 11.5 we see that $D_{z^{-1}}$ can be explicitly computed when $W$ is finite and $c_{z w_{0}}$ is known. In particular, in the setup of 7.4 with $m=2 k+2<\infty$, we can compute explicitly all $D_{z^{-1}}$ using $7.6(\mathrm{a})$. For example:

$$
\begin{aligned}
& D_{s_{1}}=\sum_{s \in[0, k-1]}\left(1-v^{2 L_{1}}+v^{4 L_{1}}-\cdots+(-1)^{s} v^{2 s L_{1}}\right) v^{-s L_{1}-s L_{2}} \\
& \times\left(T_{1_{2 s+1}}-v^{-L_{2}} T_{2_{2 s+2}}-v^{-L_{2}} T_{1_{2 s+2}}+v^{-2 L_{2}} T_{2_{2 s+3}}\right) \\
& +\left(1-v^{2 L_{1}}+v^{4 L_{1}}-\cdots+(-1)^{k} v^{2 k L_{1}}\right) v^{-k L_{1}-k L_{2}}\left(T_{1_{2 k+1}}-v^{-L_{2}} T_{2_{2 k+2}}\right) .
\end{aligned}
$$


Using this (for larger and larger $m$ ) one can deduce that an analogous formula holds in the setup of 7.4 with $m=\infty$ :

$$
\begin{aligned}
& D_{s_{1}}=\sum_{s \geq 0}\left(1-v^{2 L_{1}}+v^{4 L_{1}}-\cdots+(-1)^{s} v^{2 s L_{1}}\right) v^{-s L_{1}-s L_{2}} \\
& \times\left(T_{1_{2 s+1}}-v^{-L_{2}} T_{2_{2 s+2}}-v^{-L_{2}} T_{1_{2 s+2}}+v^{-2 L_{2}} T_{2_{2 s+3}}\right) \in \mathcal{H}^{\prime} .
\end{aligned}
$$

(We use the identification in 10.6.)

\section{The FUNCTION a}

13.1. We preserve the setup of 3.1 .

In the remainder of these notes we assume that $L(s)>0$ for all $s \in S$.

For $x, y, z$ in $W$ we define $f_{x, y, z} \in \mathcal{A}, f_{x, y, z}^{\prime} \in \mathcal{A}, h_{x, y, z} \in \mathcal{A}$ by

$$
\begin{gathered}
T_{x} T_{y}=\sum_{z \in W} f_{x, y, z} T_{z}=\sum_{z \in W} f_{x, y, z}^{\prime} c_{z} \\
c_{x} c_{y}=\sum_{z \in W} h_{x, y, z} c_{z}
\end{gathered}
$$

We have

(a) $f_{x, y, z}=\sum_{z^{\prime}} p_{z, z^{\prime}} f_{x, y, z^{\prime}}^{\prime}$

(b) $f_{x, y, z}^{\prime}=\sum_{u} q_{z, z^{\prime}}^{\prime} f_{x, y, z^{\prime}}$,

(c) $h_{x, y, z}=\sum_{x^{\prime}, y^{\prime}} p_{x^{\prime}, x} p_{y^{\prime}, y} f_{x^{\prime}, y^{\prime}, z}^{\prime}$.

All sums in (a)-(c) are finite. (a),(c) follow from the definitions; (b) follows from (a) using 10.2(a).

From 8.2, 5.6, we see that

(d) $h_{x, y, z} \neq 0 \Longrightarrow z \leq_{\mathcal{R}} x, z \leq_{\mathcal{L}} y$

(e) $h_{x, y, z}=h_{y^{-1}, x^{-1}, z^{-1}}$.

13.2. We say that $N \in \mathbf{N}$ is a bound for $W, L$ if $v^{-N} f_{x, y, z} \in \mathcal{A}_{\leq 0}$ for all $x, y, z$ in $W$. We say that $W, L$ is bounded if there exists $N \in \mathbf{N}$ such that $N$ is a bound for $W, L$. If $W$ is finite then $W, L$ is obviously bounded. More precisely:

Lemma 13.3. If $W$ is finite, then $N=L\left(w_{0}^{S}\right)$ is a bound for $W, L$.

By 10.4(a) we have $f_{x, y, z}=\tau\left(T_{x} T_{y} T_{z^{-1}}\right)$. By 10.4(c) we have $\tau\left(T_{x} T_{y} T_{z^{-1}}\right) \in$ $v^{L\left(w_{0}^{S}\right)} \mathbf{Z}\left[v^{-1}\right]$. The lemma is proved.

13.4. Conjecture. In the general case $W, L$ admits a bound $N=\max _{I} L\left(w_{0}^{I}\right)$ where $I$ runs over the subsets of $S$ such that $W_{I}$ is finite.

For $W$ is tame this is proved in [L6, 7.2] assuming that $L=l$, but the same proof remains valid without the assumption $L=l$.

We illustrate this in the setup of 7.1 with $m=\infty$. For $a, b \in\{1,2\}$ and $k>0, k^{\prime}>0$, we have 
$T_{a_{k}} T_{b_{k^{\prime}}}=T_{a_{k+k^{\prime}}}$ if $b=a+k \bmod 2$,

$T_{a_{k}} T_{b_{k^{\prime}}}=T_{a_{k} b_{k^{\prime}}}+\sum_{u \in\left[1, \min \left(k, k^{\prime}\right)\right]} \xi_{b+u-1} T_{a_{k+k^{\prime}-2 u+1}}$ if $b=a+k+1 \bmod 2$;

here, for $n \in \mathbf{Z}$ we set $\xi_{n}=v^{L_{1}}-v^{-L_{1}}$ if $n$ is odd and $\xi_{n}=v^{L_{2}}-v^{-L_{2}}$ if $n$ is even. We see that, in this case, $\max \left(L_{1}, L_{2}\right)$ is a bound for $W, L$.

Lemma 13.5. Assume that $N$ is a bound for $W, L$. Then, for any $x, y, z$ in $W$ we have

(a) $v^{-N} f_{x, y, z}^{\prime} \in \mathcal{A}_{\leq 0}$,

(b) $v^{-N} h_{x, y, z} \in \mathcal{A}_{\leq 0}$.

(a) follows from 13.1(b) since $q_{z, z^{\prime}}^{\prime} \in \mathcal{A}_{\leq 0}$. (b) follows from (a) and 13.1(c) since $p_{x^{\prime}, x} \in \mathcal{A}_{\leq 0}, p_{y^{\prime}, y} \in \mathcal{A}_{\leq 0}$.

13.6. In the remainder of this section we assume that $W, L$ is bounded and $N$ is a bound for $W, L$. By 13.5(b), for any $z \in W$ there exists a unique integer $\mathbf{a}(z) \in[0, N]$ such that

(a) $h_{x, y, z} \in v^{\mathbf{a}(z)} \mathbf{Z}\left[v^{-1}\right]$ for all $x, y \in W$,

(b) $h_{x, y, z} \notin v^{\mathbf{a}(z)-1} \mathbf{Z}\left[v^{-1}\right]$ for some $x, y \in W$.

(We use that $h_{1, z, z}=1$.) We then have for any $x, y, z$ :

(c) $h_{x, y, z}=\gamma_{x, y, z^{-1}} v^{\mathbf{a}(z)} \bmod v^{\mathbf{a}(z)-1} \mathbf{Z}\left[v^{-1}\right]$

where $\gamma_{x, y, z^{-1}} \in \mathbf{Z}$ is well defined; moreover, for any $z \in W$ there exists $x, y$ such that $\gamma_{x, y, z^{-1}} \neq 0$.

For any $x, y, z$ we have

(d) $f_{x, y, z}^{\prime}=\gamma_{x, y, z^{-1}} v^{\mathbf{a}(z)} \bmod v^{\mathbf{a}(z)-1} \mathbf{Z}\left[v^{-1}\right]$.

This is proved (for fixed $z$ ) by induction on $l(x)+l(y)$ using (c) and 13.1(c). (Note that $p_{x^{\prime}, x} p_{y^{\prime}, y}$ is 1 if $x^{\prime}=x, y^{\prime}=y$ and is in $\mathcal{A}_{<0}$ otherwise.)

Proposition 13.7. (a) $\mathbf{a}(1)=0$.

(b) If $z \in W-\{1\}$, then $\mathbf{a}(z) \geq \min _{s \in S} L(s)>0$.

We prove (a). Let $x, y \in W$. Assume first that $y \neq 1$. We can find $s \in S$ such that $y s<y$. Then $c_{y} \in{ }^{s} \mathcal{H}$. Since ${ }^{s} \mathcal{H}$ is a left ideal (see 8.4) we have $c_{x} c_{y} \in{ }^{s} \mathcal{H}$. Since $s 1>1$, from the definition of ${ }^{s} \mathcal{H}$ it then follows that $h_{x, y, 1}=0$.

Similarly, if $x \neq 1$, then $h_{x, y, 1}=0$. Since $h_{1,1,1}=1$, (a) follows.

In the setup of (b) we can find $s \in S$ such that $s z<z$. By 6.6(b) we have $h_{s, z, z}=v_{s}+v_{s}^{-1}$. This shows that $\mathbf{a}(z) \geq L(s)>0$. The proposition is proved.

Proposition 13.8. Assume that $W$ is finite.

(a) We have $\mathbf{a}\left(w_{0}\right)=L\left(w_{0}\right)$.

(b) For any $w \in W-\left\{w_{0}\right\}$ we have $\mathbf{a}(w)<L\left(w_{0}\right)$.

For any $w \in W$ we have by definition $\mathbf{a}(w) \leq N$ where $N$ is a bound for $W, L$; by 13.3 we can take $N=L\left(w_{0}\right)$, hence $\mathbf{a}(w) \leq L\left(w_{0}\right)$.

We prove (a). From 6.6(b) we see that $T_{s} c_{w_{0}}=v_{s} c_{w_{0}}$ for any $s \in S$. Using this and 12.2 , we see that 
hence

$c_{w_{0}} c_{w_{0}}=\sum_{y \in W} v^{-L\left(y w_{0}\right)} v^{L(y)} c_{w_{0}}$

$$
h_{w_{0}, w_{0}, w_{0}}=\sum_{y \in W} v^{-L\left(w_{0}\right)} v^{2 L(y)} \in v^{L\left(w_{0}\right)} \bmod v^{L\left(w_{0}\right)-1} \mathbf{Z}\left[v^{-1}\right] .
$$

It follows that $\mathbf{a}\left(w_{0}\right) \geq L\left(w_{0}\right)$. Hence $\mathbf{a}\left(w_{0}\right)=L\left(w_{0}\right)$. This proves (a).

We prove (b). Let $z \in W$ be such that $\mathbf{a}(z)=L\left(w_{0}\right)$. We must prove that $z=w_{0}$. By 13.6(d), we can find $x, y$ such that

$$
f_{x, y, z}^{\prime}=b v^{L\left(w_{0}\right)}+\text { strictly smaller powers of } v
$$

where $b \in \mathbf{Z}-\{0\}$. For any $z^{\prime} \neq z$ we have $f_{x, y, z^{\prime}}^{\prime} \in v^{L\left(w_{0}\right)} \mathbf{Z}\left[v^{-1}\right]$ (by 13.6 and the first sentence in the proof). Since $p_{z, z^{\prime}}=1$ for $z=z^{\prime}$ and $p_{z, z^{\prime}} \in \mathcal{A}_{<0}$ for $z^{\prime}<z$, we see that the equality $f_{x, y, z}=\sum_{z^{\prime}} p_{z, z^{\prime}} f_{x, y, z^{\prime}}^{\prime}$ (see 13.1(a)) implies that

$$
f_{x, y, z}=b v^{L\left(w_{0}\right)}+\text { strictly smaller powers of } v
$$

with $b \neq 0$. Now $f_{x, y, z}=\tau\left(T_{x} T_{y} T_{z^{-1}}\right)$. Using now $10.4(\mathrm{c})$ we see that

$$
\min \left(L(x), L(y), L\left(z^{-1}\right)\right)=L\left(w_{0}\right) .
$$

It follows that $x=y=z^{-1}=w_{0}$. The proposition is proved.

Proposition 13.9. (a) For any $z \in W$ we have $\mathbf{a}(z)=\mathbf{a}\left(z^{-1}\right)$.

(b) For any $x, y, z \in W$ we have $\gamma_{x, y, z}=\gamma_{y^{-1}, x^{-1}, z^{-1}}$.

(a),(b) follow from 13.1(e).

13.10. We show that, in the setup of 7.1 with $m=\infty$ and $L_{2} \geq L_{1}$, the function $\mathbf{a}: W \rightarrow \mathbf{N}$ is given as follows:

(a) $\mathbf{a}(1)=0$

(b) $\mathbf{a}\left(1_{1}\right)=L_{1}, \mathbf{a}\left(2_{1}\right)=L_{2}$,

(c) $\mathbf{a}\left(1_{k}\right)=\mathbf{a}\left(2_{k}\right)=L_{2}$ if $k \geq 2$.

Now (a) is contained in 13.7(a). If $s_{2} z<z$ then, by the proof of 13.7 (b) we have $\mathbf{a}(z) \geq L_{2}$. By $13.4, L_{2}$ is a bound for $W, L$ hence $\mathbf{a}(z) \leq L_{2}$ so that $\mathbf{a}(z)=L_{2}$. If $z s_{2}<z$ then the previous argument is applicable to $z^{-1}$. Using 13.9 , we see that $\mathbf{a}(z)=\mathbf{a}\left(z^{-1}\right)=L_{2}$.

Assume next that $z=1_{2 k+1}$ where $k \geq 1$. By 7.5, 7.6, we have

$$
c_{1_{2}} c_{2_{2 k}}=c_{1} c_{2} c_{2_{2 k}}=\left(v^{L_{2}}+v^{-L_{2}}\right) c_{1} c_{2_{2 k}}=\left(v^{L_{2}}+v^{-L_{2}}\right) c_{1_{2 k+1}}
$$

hence $h_{1_{2}, 2_{2 k}, z}=v^{L_{2}}+v^{-L_{2}}$. Thus, $\mathbf{a}(z) \geq L_{2}$. By 13.4 we have $\mathbf{a}(z) \leq L_{2}$ hence $\mathbf{a}(z)=L_{2}$.

It remains to consider the case where $z=s_{1}$. Assume first that $L_{1}=L_{2}$. Then $\mathbf{a}\left(s_{1}\right) \leq L_{1}$ by 13.4 and $\mathbf{a}\left(s_{1}\right) \geq L_{1}$ by $13.7\left(\right.$ b). Hence $\mathbf{a}\left(s_{1}\right)=L_{1}$.

Assume next that $L_{1}<L_{2}$. Then $\mathcal{I}=\sum_{w \in W-\left\{1, s_{1}\right\}} \mathcal{A} c_{w}$ is a two-sided ideal $\mathcal{I}$ of $\mathcal{H}$ (see 8.8). Hence if $x$ or $y$ is in $W-\left\{1, s_{1}\right\}$, then $c_{x} c_{y} \in \mathcal{I}$ and $h_{x, y, s_{1}}=0$. Using

$$
h_{1,1, s_{1}}=0, h_{1, s_{1}, s_{1}}=h_{s_{1}, 1, s_{1}}=1, h_{s_{1}, s_{1}, s_{1}}=v^{L_{1}}+v^{-L_{1}}
$$

we see that $\mathbf{a}\left(s_{1}\right)=L_{1}$. Thus, (a),(b),(c) are established. 
13.11. In this subsection we assume that we are in the setup of 7.1 with $4 \leq m<$ $\infty$ and $L_{2}>L_{1}$. By 7.8 , we have

$$
h_{2_{m-1}, 2_{m-1}, 2_{m-1}}=(-1)^{(m-2) / 2} v^{\left(m L_{2}-(m-2) L_{1}\right) / 2}+\text { strictly smaller powers of } v \text {. }
$$

Hence $\mathbf{a}\left(2_{m-1}\right) \geq\left(m L_{2}-(m-2) L_{1}\right) / 2$.

One can show that the function $\mathbf{a}: W \rightarrow \mathbf{N}$ is given as follows:

$$
\begin{aligned}
& \mathbf{a}(1)=0, \\
& \mathbf{a}\left(1_{1}\right)=L_{1}, \mathbf{a}\left(2_{1}\right)=L_{2}, \\
& \mathbf{a}\left(1_{m-1}\right)=L_{2}, \mathbf{a}\left(2_{m-1}\right)=\left(m L_{2}-(m-2) L_{1}\right) / 2, \\
& \mathbf{a}\left(2_{m}\right)=m\left(L_{1}+L_{2}\right) / 2, \\
& \mathbf{a}\left(1_{k}\right)=\mathbf{a}\left(2_{k}\right)=L_{2} \text { if } 1<k<m-1 .
\end{aligned}
$$

This remains true in the case where $L_{1}=L_{2}$.

13.12. Let $w \in W$. Let $Z(w)$ be the set of all $y \in W$ such that $w=u y u^{\prime}$ for some $u, u^{\prime} \in W$ with $l(w)=l(u)+l(y)+l\left(u^{\prime}\right)$ and $y \in W_{I}$ for some $I \subset S$ with $W_{I}$ finite. Let $\mathbf{a}^{\prime}(w)=\max _{y \in Z(w)} \mathbf{a}(y)$. We conjecture that

$$
\mathbf{a}(w)=\mathbf{a}^{\prime}(w)
$$

For $u, u^{\prime}, y$ as above we have $w=u y u^{\prime} \leq_{\mathcal{L}} y u^{\prime} \leq_{\mathcal{R}} y$ hence $w \leq_{\mathcal{L} \mathcal{R}} y$. Hence for some $y \in Z(w)$ we have $w \leq_{\mathcal{L R}} y$ and $\mathbf{a}(w)=\mathbf{a}(y)$ (assuming that (a) holds). If we assume also that P11 in 14.2 holds, we deduce that $w \sim_{\mathcal{L R}} y$. Hence any two-sided cell of $W$ would meet some finite $W_{I}$; this would imply that there are only finitely many two-sided cells in $W$. (Compare 18.2.)

On the other hand, the number of left cells in $W$ can be infinite for some non-tame $W$ with $L=l$ (Bédard [Be])

\section{Conjectures}

14.1. We preserve the setup of 3.1. In this section we assume that $W, L$ is bounded.

For $n \in \mathbf{Z}$ define $\pi_{n}: \mathcal{A} \rightarrow \mathbf{Z}$ by $\pi_{n}\left(\sum_{k \in \mathbf{Z}} a_{k} v^{k}\right)=a_{n}$.

For $z \in W$ we define an integer $\Delta(z) \geq 0$ by

$$
p_{1, z}=n_{z} v^{-\Delta(z)}+\text { strictly smaller powers of } v, n_{z} \in \mathbf{Z}-\{0\} \text {. }
$$

Note that $\Delta(z)=\Delta\left(z^{-1}\right)$ and $\Delta(1)=0,0<\Delta(z) \leq L(z)$ for $z \neq 1$ (see 5.4). Let

$$
\mathcal{D}=\{z \in W ; \mathbf{a}(z)=\Delta(z)\}
$$

Clearly, $z \in \mathcal{D} \Longrightarrow z^{-1} \in \mathcal{D}$. 
Conjectures 14.2. The following properties hold.

P1. For any $z \in W$ we have $\mathbf{a}(z) \leq \Delta(z)$.

P2. If $d \in \mathcal{D}$ and $x, y \in W$ satisfy $\gamma_{x, y, d} \neq 0$, then $x=y^{-1}$.

P3. If $y \in W$, there exists a unique $d \in \mathcal{D}$ such that $\gamma_{y^{-1}, y, d} \neq 0$.

P4. If $z^{\prime} \leq_{\mathcal{L R}} z$ then $\mathbf{a}\left(z^{\prime}\right) \geq \mathbf{a}(z)$. Hence, if $z^{\prime} \sim_{\mathcal{L R}} z$, then $\mathbf{a}\left(z^{\prime}\right)=\mathbf{a}(z)$.

P5. If $d \in \mathcal{D}, y \in W, \gamma_{y^{-1}, y, d} \neq 0$, then $\gamma_{y^{-1}, y, d}=n_{d}= \pm 1$.

P6. If $d \in \mathcal{D}$, then $d^{2}=1$.

P7. For any $x, y, z \in W$ we have $\gamma_{x, y, z}=\gamma_{y, z, x}$.

P8. Let $x, y, z \in W$ be such that $\gamma_{x, y, z} \neq 0$. Then $x \sim_{\mathcal{L}} y^{-1}, y \sim_{\mathcal{L}} z^{-1}$, $z \sim \mathcal{L} x^{-1}$.

P9. If $z^{\prime} \leq_{\mathcal{L}} z$ and $\mathbf{a}\left(z^{\prime}\right)=\mathbf{a}(z)$ then $z^{\prime} \sim_{\mathcal{L}} z$.

P10. If $z^{\prime} \leq_{\mathcal{R}} z$ and $\mathbf{a}\left(z^{\prime}\right)=\mathbf{a}(z)$ then $z^{\prime} \sim_{\mathcal{R}} z$.

P11. If $z^{\prime} \leq_{\mathcal{L R}} z$ and $\mathbf{a}\left(z^{\prime}\right)=\mathbf{a}(z)$ then $z^{\prime} \sim_{\mathcal{L R}} z$.

P12. Let $I \subset S$. If $y \in W_{I}$, then $\mathbf{a}(y)$ computed in terms of $W_{I}$ is equal to $\mathbf{a}(y)$ computed in terms of $W$.

P13. Any left cell $\Gamma$ of $W$ contains a unique element $d \in \mathcal{D}$. We have $\gamma_{x^{-1}, x, d} \neq$ 0 for all $x \in \Gamma$.

P14. For any $z \in W$ we have $z \sim \mathcal{L R} z^{-1}$.

P15. Let $v^{\prime}$ be a second indeterminate and let $h_{x, y, z}^{\prime} \in \mathbf{Z}\left[v^{\prime}, v^{\prime-1}\right]$ be obtained from $h_{x, y, z}$ by the substitution $v \mapsto v^{\prime}$. If $x, x^{\prime}, y, w \in W$ satisfy $\mathbf{a}(w)=\mathbf{a}(y)$, then $\sum_{y^{\prime}} h_{w, x^{\prime}, y^{\prime}}^{\prime} h_{x, y^{\prime}, y}=\sum_{y^{\prime}} h_{x, w, y^{\prime}} h_{y^{\prime}, x^{\prime}, y}^{\prime}$

In $\S 15-\S 17$ we will verify the conjectures above in a number of cases.

14.3. We consider the following auxiliary statement.

$\tilde{P}$. Let $x, y, z, z^{\prime} \in W$ be such that $\gamma_{x, y, z^{-1}} \neq 0, z^{\prime} \leftarrow_{\mathcal{L}} z$. Then there exists $x^{\prime} \in W$ such that $\pi_{\mathbf{a}(z)}\left(h_{x^{\prime}, y, z^{\prime}}\right) \neq 0$. In particular, $\mathbf{a}\left(z^{\prime}\right) \geq \mathbf{a}(z)$.

In this section we will show, that, if P1-P3 and $\tilde{P}$ are assumed to be true, then P4-P14 are automatically true. The arguments follow [L6],[L8].

14.4. $\tilde{P} \Longrightarrow P 4$. Let $z^{\prime}, z$ be as in $\mathrm{P} 4$. We can assume that $z \leftarrow \mathcal{L} z^{\prime}$ or $z \leftarrow \mathcal{R} z^{\prime}$. In the first case, from $\tilde{P}$ we get $\mathbf{a}\left(z^{\prime}\right) \geq \mathbf{a}(z)$. (We can find $x, y$ such that $\gamma_{x, y, z^{-1}} \neq 0$.) In the second case, from $\tilde{P}$ we get $\mathbf{a}\left(z^{\prime-1}\right) \geq \mathbf{a}\left(z^{-1}\right)$ hence $\mathbf{a}\left(z^{\prime}\right) \geq \mathbf{a}(z)$.

14.5. $P 1, P 3 \Longrightarrow P 5$. Let $x, y \in W$. Applying $\tau$ to $c_{x} c_{y}=\sum_{z \in W} h_{x, y, z} c_{z}$ gives

$$
\sum_{z} h_{x, y, z} p_{1, z}=\sum_{x^{\prime}, y^{\prime}} p_{x^{\prime}, x} p_{y^{\prime}, y} \tau\left(T_{x^{\prime}} T_{y^{\prime}}\right)=\sum_{x^{\prime}, y^{\prime}} p_{x^{\prime}, x} p_{y^{\prime}, y} \delta_{x^{\prime} y^{\prime}, 1}=\sum_{x^{\prime}} p_{x^{\prime}, x} p_{x^{\prime-1}, y}
$$

hence

$$
\sum_{z \in W} h_{x, y, z} p_{1, z}=\delta_{x y, 1} \bmod v^{-1} \mathbf{Z}\left[v^{-1}\right]
$$


We take $x=y^{-1}$ and note that $h_{y^{-1}, y, z} \in v^{\mathbf{a}(z)} \mathbf{Z}\left[v^{-1}\right], p_{1, z} \in v^{-\Delta(z)} \mathbf{Z}\left[v^{-1}\right]$, hence

$$
h_{y^{-1}, y, z} p_{1, z} \in v^{\mathbf{a}(z)-\Delta(z)} \mathbf{Z}\left[v^{-1}\right]
$$

The same argument shows that, if $z \in \mathcal{D}$, then

$$
h_{y^{-1}, y, z} p_{1, z} \in \gamma_{y^{-1}, y, z^{-1}} n_{z}+\mathcal{A}_{<0}
$$

If $z \notin \mathcal{D}$ then, by $\mathrm{P} 1$, we have $\mathbf{a}(z)-\Delta(z)<0$ so that $h_{y^{-1}, y, z} p_{1, z} \in \mathcal{A}_{<0}$. We see that

$$
\sum_{z \in W} h_{y^{-1}, y, z} p_{1, z}=\sum_{z \in \mathcal{D}} \gamma_{y^{-1}, y, z^{-1}} n_{z} \quad \bmod \mathcal{A}_{<0}
$$

Comparison with (a) gives $\sum_{z \in \mathcal{D}} \gamma_{y^{-1}, y, z^{-1}} n_{z}=1$. Equivalently,

$$
\sum_{z \in \mathcal{D}} \gamma_{y^{-1}, y, z} n_{z}=1
$$

Using this and P3 we see that, in the setup of P5 we have $\gamma_{y^{-1}, y, d} n_{d}=1$. Since $\gamma_{y^{-1}, y, d}, n_{d}$ are integers, we must have $\gamma_{y^{-1}, y, d}=n_{d}= \pm 1$.

14.6. $P 2, P 3 \Longrightarrow P 6$. We can find $x, y$ such that $\gamma_{x, y, d} \neq 0$. By P2, we have $x=y^{-1}$ so that $\gamma_{y^{-1}, y, d} \neq 0$. This implies $\gamma_{y^{-1}, y, d^{-1}} \neq 0$. (See 13.9(b)). We have $d^{-1} \in \mathcal{D}$. By the uniqueness in P3 we have $d=d^{-1}$.

14.7. $P$ 2, $P 3, P 4, P 5 \Longrightarrow P$ \% . We first prove the following statement.

(a) Let $x, y, z \in W, d \in \mathcal{D}$ be such that $\gamma_{x, y, z} \neq 0, \gamma_{z^{-1}, z, d} \neq 0$, a $(d)=\mathbf{a}(z)$. Then $\gamma_{x, y, z}=\gamma_{y, z, x}$.

Let $n=\mathbf{a}(d)$. From $\gamma_{x, y, z} \neq 0$ we deduce $h_{x, y, z^{-1}} \neq 0$ hence $z^{-1} \leq_{\mathcal{R}} x$, hence $n=\mathbf{a}(z)=\mathbf{a}\left(z^{-1}\right) \geq \mathbf{a}(x)$ (see $\left.\mathrm{P} 4\right)$. Computing the coefficient of $c_{d}$ in two ways, we obtain

$$
\sum_{z^{\prime}} h_{x, y, z^{\prime}} h_{z^{\prime}, z, d}=\sum_{x^{\prime}} h_{x, x^{\prime}, d} h_{y, z, x^{\prime}}
$$

Now $h_{z^{\prime}, z, d} \neq 0$ implies $d \leq_{\mathcal{R}} z^{\prime}$ hence $\mathbf{a}\left(z^{\prime}\right) \leq \mathbf{a}(d)=n$ (see P4); similarly, $h_{x, x^{\prime}, d} \neq 0$ implies $d \leq_{\mathcal{L}} x^{\prime}$ hence $\mathbf{a}\left(x^{\prime}\right) \leq \mathbf{a}(d)=n$. Thus we have

$$
\sum_{z^{\prime} ; \mathbf{a}\left(z^{\prime}\right) \leq n} h_{x, y, z^{\prime}} h_{z^{\prime}, z, d}=\sum_{x^{\prime} ; \mathbf{a}\left(x^{\prime}\right) \leq n} h_{x, x^{\prime}, d} h_{y, z, x^{\prime}}
$$

By P2 and our assumptions, the left hand side is

$$
\gamma_{x, y, z} \gamma_{z^{-1}, z, d} v^{2 n}+\text { strictly smaller powers of } v \text {. }
$$

Similarly, the right hand side is

$$
\gamma_{x, x^{-1}, d} \pi_{n}\left(h_{y, z, x^{-1}}\right) v^{2 n}+\text { strictly smaller powers of } v \text {. }
$$


Hence $\gamma_{x, x^{-1}, d} \pi_{n}\left(h_{y, z, x^{-1}}\right)=\gamma_{x, y, z} \gamma_{z^{-1}, z, d} \neq 0$. Thus,

$$
\gamma_{x, x^{-1}, d} \neq 0, \pi_{n}\left(h_{y, z, x^{-1}}\right) \neq 0 .
$$

We see that $\mathbf{a}\left(x^{-1}\right) \geq n$. But we have also $\mathbf{a}(x) \leq n$ hence $\mathbf{a}(x)=n$ and $\pi_{n}\left(h_{y, z, x^{-1}}\right)=\gamma_{y, z, x}$. Since $\gamma_{x, x^{-1}, d} \neq 0$, we have (by P5) $\gamma_{x, x^{-1}, d}=\gamma_{z^{-1}, z, d}$. Using this and $\gamma_{x, x^{-1}, d} \gamma_{y, z, x}=\gamma_{x, y, z} \gamma_{z^{-1}, z, d}$ we deduce $\gamma_{y, z, x}=\gamma_{x, y, z}$, as required.

Next we prove the following statement.

(b) Let $z \in W, d \in \mathcal{D}$ be such that $\gamma_{z^{-1}, z, d} \neq 0$. Then $\mathbf{a}(z)=\mathbf{a}(d)$.

We shall assume that (b) holds whenever $\mathbf{a}(z)>N_{0}$ and we shall deduce that it also holds when $\mathbf{a}(z)=N_{0}$. (This will prove (b) by descending induction on $\mathbf{a}(z)$ since $\mathbf{a}(z)$ is bounded above.) Assume that $\mathbf{a}(z)=N_{0}$. From $\gamma_{z^{-1}, z, d}= \pm 1$ we deduce that $h_{z^{-1}, z, d^{-1}} \neq 0$ hence $d^{-1} \leq_{\mathcal{L}} z^{-1}$ hence $\mathbf{a}\left(d^{-1}\right) \geq \mathbf{a}\left(z^{-1}\right)$ (see P4) and $\mathbf{a}(d) \geq \mathbf{a}(z)$. Assume that $\mathbf{a}(d)>\mathbf{a}(z)$, that is, $\mathbf{a}(d)>N_{0}$. Let $d^{\prime} \in \mathcal{D}$ be such that $\gamma_{d^{-1}, d, d^{\prime}} \neq 0$ (see P3). By the induction hypothesis applied to $d, d^{\prime}$ instead of $z, d$, we have $\mathbf{a}(d)=\mathbf{a}\left(d^{\prime}\right)$. From $\gamma_{z^{-1}, z, d} \neq 0, \gamma_{d^{-1}, d, d^{\prime}} \neq 0, \mathbf{a}(d)=\mathbf{a}\left(d^{\prime}\right)$, we deduce (using (a)) that $\gamma_{z, d, z^{-1}}=\gamma_{z^{-1}, z, d}$. Hence $\gamma_{z, d, z^{-1}} \neq 0$. It follows that $h_{z, d, z} \neq 0$, hence $z \leq_{\mathcal{L}} d$, hence $\mathbf{a}(z) \geq \mathbf{a}(d)$ (see $\left.\mathrm{P} 4\right)$. This contradicts the assumption $\mathbf{a}(d)>\mathbf{a}(z)$. Hence we must have $\mathbf{a}(z)=\mathbf{a}(d)$, as required.

We now prove P7. Assume first that $\gamma_{x, y, z} \neq 0$. Let $d \in \mathcal{D}$ be such that $\gamma_{z^{-1}, z, d} \neq 0$ (see P3). By (b) we have $\mathbf{a}(z)=\mathbf{a}(d)$. Using (a) we then have $\gamma_{x, y, z}=\gamma_{y, z, x}$. Assume next that $\gamma_{x, y, z}=0$; we must show that $\gamma_{y, z, x}=0$. We assume that $\gamma_{y, z, x} \neq 0$. By the first part of the proof, we have

$$
\gamma_{y, z, x} \neq 0 \Longrightarrow \gamma_{y, z, x}=\gamma_{z, x, y} \neq 0 \Longrightarrow \gamma_{z, x, y}=\gamma_{x, y, z} \neq 0,
$$

a contradiction.

14.8. $P$ P $\Longrightarrow P 8$. If $\gamma_{x, y, z} \neq 0$, then $h_{x, y, z^{-1}} \neq 0$, hence $z^{-1} \leq_{\mathcal{L}} y, z \leq_{\mathcal{L}} x^{-1}$. By P7 we also have $\gamma_{y, z, x} \neq 0$ (hence $x^{-1} \leq_{\mathcal{L}} z, x \leq_{\mathcal{L}} y^{-1}$ ) and $\gamma_{z, x, y} \neq 0$ (hence $\left.y^{-1} \leq_{\mathcal{L}} x, y \leq_{\mathcal{L}} z^{-1}\right)$. Thus, we have $x \sim_{\mathcal{L}} y^{-1}, y \sim_{\mathcal{L}} z^{-1}, z \sim_{\mathcal{L}} x^{-1}$.

14.9. $\tilde{P}, P 4, P 8 \Longrightarrow P 9$. We can find a sequence $z^{\prime}=z_{0}, z_{1}, \ldots, z_{n}=z$ such that for any $j \in[1, n]$ we have $z_{j-1} \leftarrow \mathcal{L} z_{j}$. By P4 we have $\mathbf{a}\left(z^{\prime}\right)=\mathbf{a}\left(z_{0}\right) \geq \mathbf{a}\left(z_{1}\right) \geq$ $\cdots \geq \mathbf{a}\left(z_{n}\right)=\mathbf{a}(z)$. Since $\mathbf{a}(z)=\mathbf{a}\left(z^{\prime}\right)$, we have $\mathbf{a}\left(z^{\prime}\right)=\mathbf{a}\left(z_{0}\right)=\mathbf{a}\left(z_{1}\right)=\cdots=$ $\mathbf{a}\left(z_{n}\right)=\mathbf{a}(z)$. Thus, it suffices to show that, if $z^{\prime} \leftarrow_{\mathcal{L}} z$ and $\mathbf{a}\left(z^{\prime}\right)=\mathbf{a}(z)$, then $z^{\prime} \sim_{\mathcal{L}} z$. Let $x, y \in W$ be such that $\gamma_{x, y, z^{-1}} \neq 0$. By $\tilde{P}$, there exists $x^{\prime} \in W$ such that $\pi_{\mathbf{a}(z)}\left(h_{x^{\prime}, y, z^{\prime}}\right) \neq 0$. Since $\mathbf{a}\left(z^{\prime}\right)=\mathbf{a}(z)$, we have $\gamma_{x^{\prime}, y, z^{\prime-1}} \neq 0$. From $\gamma_{x, y, z^{-1}} \neq 0, \gamma_{x^{\prime}, y, z^{\prime-1}} \neq 0$ we deduce, using P8, that $y \sim_{\mathcal{L}} z, y \sim_{\mathcal{L}} z^{\prime}$, hence $z \sim \sim_{\mathcal{L}} z^{\prime}$

14.10. $P 9 \Longrightarrow P 10$. We apply P9 to $z^{-1}, z^{\prime-1}$.

14.11. $P 4, P 9, P 10 \Longrightarrow P 11$. We can find a sequence $z^{\prime}=z_{0}, z_{1}, \ldots, z_{n}=z$ such that for any $j \in[1, n]$ we have $z_{j-1} \leq_{\mathcal{L}} z_{j}$ or $z_{j-1} \leq_{\mathcal{R}} z_{j}$. By P4, we have $\mathbf{a}\left(z^{\prime}\right)=\mathbf{a}\left(z_{0}\right) \geq \mathbf{a}\left(z_{1}\right) \geq \cdots \geq \mathbf{a}\left(z_{n}\right)=\mathbf{a}(z)$. Since $\mathbf{a}(z)=\mathbf{a}\left(z^{\prime}\right)$, we have $\mathbf{a}\left(z^{\prime}\right)=\mathbf{a}\left(z_{0}\right)=\mathbf{a}\left(z_{1}\right)=\cdots=\mathbf{a}\left(z_{n}\right)=\mathbf{a}(z)$. Applying P9 or P10 to $z_{j-1}, z_{j}$ we obtain $z_{j-1} \sim_{\mathcal{L}} z_{j}$ or $z_{j-1} \sim_{\mathcal{R}} z_{j}$. Hence $z^{\prime} \sim_{\mathcal{L} \mathcal{R}} z$. 
14.12. $P 3, P 4, P 8$ for $W$ and $W_{I} \Longrightarrow P 12$. We write $\mathbf{a}_{I}: W_{I} \rightarrow \mathbf{N}$ for the a-function defined in terms of $W_{I}$. For $x, y, z \in W_{I}$, we write $h_{x, y, z}^{I}, \gamma_{x, y, z}^{I}$ for the analogues of $h_{x, y, z}, \gamma_{x, y, z}$ when $W$ is replaced by $W_{I}$. Let $\mathcal{H}_{I} \subset \mathcal{H}$ be as in 9.9.

Let $d \in \mathcal{D}$ be such that $\gamma_{y^{-1}, y, d} \neq 0$. (See P3.) Then $\pi_{\mathbf{a}(d)}\left(h_{y^{-1}, y, d^{-1}}\right) \neq 0$. Now $c_{y^{-1}} c_{y} \in \mathcal{H}_{I}$ hence $d \in W_{I}$ and $\pi_{\mathbf{a}(d)}\left(h_{y^{-1}, y, d^{-1}}^{I}\right) \neq 0$. Thus, $\mathbf{a}_{I}\left(d^{-1}\right) \geq \mathbf{a}\left(d^{-1}\right)$. The reverse inequality is obvious hence $\mathbf{a}_{I}(d)=\mathbf{a}(d)$. We see that $\gamma_{y^{-1}, y, d}^{I} \neq 0$. From $\mathrm{P} 8$ we see that $y \sim_{\mathcal{L}} d$ (relative to $W_{I}$ ) and $y \sim_{\mathcal{L}} d$ (relative to $W$ ). From $\mathrm{P} 4$ we deduce that $\mathbf{a}_{I}(y)=\mathbf{a}_{I}(d)$ and $\mathbf{a}(y)=\mathbf{a}(d)$. It follows that $\mathbf{a}(y)=\mathbf{a}_{I}(y)$.

14.13. $\tilde{P}, P 2, P 3, P 4, P 6, P 8 \Longrightarrow P 13$. If $x \in \Gamma$ then, by $\mathrm{P} 3$, there exists $d \in \mathcal{D}$ such that $\gamma_{x^{-1}, x, d} \neq 0$. By P8 we have $x \sim_{\mathcal{L}} d^{-1}$ hence $d^{-1} \in \Gamma$. By P6, we have $d=d^{-1}$ hence $d \in \Gamma$. It remains to prove the uniqueness of $d$. Let $d^{\prime}, d^{\prime \prime}$ be elements of $\mathcal{D} \cap \Gamma$. We must prove that $d^{\prime}=d^{\prime \prime}$. We can find $x^{\prime}, y^{\prime}, x^{\prime \prime}, y^{\prime \prime}$ such that $\gamma_{x^{\prime}, y^{\prime}, d^{\prime}} \neq 0, \gamma_{x^{\prime \prime}, y^{\prime \prime}, d^{\prime \prime}} \neq 0$. By P2, we have $x^{\prime}=y^{\prime-1}, x^{\prime \prime}=y^{\prime \prime-1}$. By P8, we have $y^{\prime} \sim_{\mathcal{L}} d^{\prime-1}=d^{\prime}$ and $y^{\prime \prime} \sim_{\mathcal{L}} d^{\prime \prime-1}=d^{\prime \prime}$, hence $y^{\prime}, y^{\prime \prime} \in \Gamma$. By the definition of left cells, we can find a sequence $y^{\prime}=x_{0}, x_{1}, \ldots, x_{n}=y^{\prime \prime}$ such that for any $j \in[1, n]$ we have $x_{j-1} \leftarrow_{\mathcal{L}} x_{j}$. Since $y^{\prime} \sim_{\mathcal{L}} y^{\prime \prime}$, we have $x_{j} \in \Gamma$ for all $j$. For $j \in[1, n-1]$ let $d_{j} \in \mathcal{D}$ be such that $\gamma_{x_{j}^{-1}, x_{j}, d_{j}} \neq 0$. Let $d_{0}=d^{\prime}, d_{n}=d^{\prime \prime}$. As in the beginning of the proof, we have $d_{j} \in \Gamma$ for each $j$. Let $j \in[1, n]$. Since $x_{j-1} \leftarrow \mathcal{L} x_{j}$, we have (by P8) $\gamma_{x_{j}, d_{j}, x_{j}^{-1}} \neq 0$. Applying $\tilde{P}$ to $x_{j}, d_{j}, x_{j}, x_{j-1}$ instead of $x, y, z, z^{\prime}$, we see that there exists $u$ such that $\pi_{\mathbf{a}\left(x_{j}\right)}\left(h_{u, d_{j}, x_{j-1}}\right) \neq 0$. Since $x_{j-1} \sim x_{j}$, we have $\mathbf{a}\left(x_{j-1}\right)=\mathbf{a}\left(x_{j}\right)$ (see P4), hence $\pi_{\mathbf{a}\left(x_{j}\right)}\left(h_{u, d_{j}, x_{j-1}}\right)=\gamma_{u, d_{j}, x_{j-1}^{-1}} \neq 0$. Using P8, we deduce $\gamma_{x_{j-1}^{-1}, u, d_{j}} \neq 0$. Using P2 we see that $u=x_{j-1}$ and $\gamma_{x_{j-1}^{-1}, x_{j-1}, d_{j}} \neq 0$. We have also $\gamma_{x_{j-1}^{-1}, x_{j-1}, d_{j-1}} \neq 0$ and by the uniqueness in P3, it follows that $d_{j-1}=d_{j}$. It follows that $d^{\prime}=d^{\prime \prime}$, as required.

14.14. $P 6, P 13 \Longrightarrow P 14$. By P13, we can find $d \in \mathcal{D}$ such that $z \sim_{\mathcal{L}} d$. Since $d=d^{-1}$ (see P6), it follows that $z^{-1} \sim_{\mathcal{R}} d$. Thus, $z \sim_{\mathcal{L R}} z^{-1}$.

14.15. In this subsection we reformulate conjecture $\mathrm{P} 15$, assuming that $\mathrm{P} 4, \mathrm{P} 9$, $\mathrm{P} 10$ hold. Let $\tilde{\mathcal{A}}=\mathbf{Z}\left[v, v^{-1}, v^{\prime}, v^{\prime-1}\right]$ where $v, v^{\prime}$ are indeterminates. Let $\tilde{\mathcal{H}}$ be the free $\tilde{\mathcal{A}}$-module with basis $e_{w}(w \in W)$. Let $\mathcal{H}^{\prime}, c_{w}^{\prime}, h_{x, y, z}^{\prime}$ be obtained from $\mathcal{H}, c_{w}, h_{x, y, z}$ by changing the variable $v$ to $v^{\prime}$.

On $\tilde{\mathcal{H}}$ we have a left $\mathcal{H}$-module structure given by $v^{n} c_{y} e_{w}=\sum_{x} v^{n} h_{y, w, x} e_{x}$ and a right $\mathcal{H}^{\prime}$-module structure given by $e_{w}\left(v^{\prime n} c_{y}^{\prime}\right)=\sum_{x} v^{\prime n} h_{w, y, x}^{\prime} e_{x}$. These module structures do not commute in general. For each $a \geq 0$ let $\tilde{\mathcal{H}} \geq a$ be the $\tilde{\mathcal{A}}$-submodule of $\tilde{\mathcal{H}}$ spanned by $\left\{e_{w} ; \mathbf{a}(w) \geq a\right\}$. By $\mathrm{P} 4$, this is a left $\mathcal{H}$-submodule and a right $\mathcal{H}^{\prime}$-submodule of $\tilde{\mathcal{H}}$. We have

$$
\ldots \tilde{\mathcal{H}}_{\geq 2} \subset \tilde{\mathcal{H}}_{\geq 1} \subset \tilde{\mathcal{H}}_{\geq 0}=\tilde{\mathcal{H}}
$$

and $\operatorname{gr} \tilde{\mathcal{H}}=\oplus_{a \geq 0} \tilde{\mathcal{H}}_{\geq a} / \tilde{\mathcal{H}}_{\geq a+1}$ inherits a left $\mathcal{H}$-module structure and a right $\mathcal{H}^{\prime}$ module structure from $\tilde{\mathcal{H}}$. Clearly, $\mathrm{P} 15$ is equivalent to the condition that these 
module structures on $g r \tilde{\mathcal{H}}$ commute. To check this last condition, it is enough to check that

the actions of $c_{s}, c_{s^{\prime}}^{\prime}$ commute on $g r \tilde{\mathcal{H}}$ for $s, s^{\prime} \in S$.

Let $s, s^{\prime} \in S, w \in W$. A computation using 6.6, 6.7, 8.2 shows that $\left(c_{s} e_{w}\right) c_{s^{\prime}}^{\prime}-$ $c_{s}\left(e_{w} c_{s^{\prime}}^{\prime}\right)$ is 0 if $s w<w$ or $w s^{\prime}<w$, while if $s w>w, w s^{\prime}>w$, it is

$$
\sum_{y ; s y<y, y s^{\prime}<y}\left(h_{w, s^{\prime}, y}^{\prime}\left(v_{s}+v_{s}^{-1}\right)-h_{s, w, y}\left(v_{s^{\prime}}^{\prime}+v_{s^{\prime}}^{\prime-1}\right)\right) e_{y}+\sum_{y ; s y<y, y s^{\prime}<y} \alpha_{y} e_{y}
$$

where

$$
\alpha_{y}=\sum_{y^{\prime} ; y^{\prime} s^{\prime}<y^{\prime}<s y^{\prime}} h_{w, s^{\prime}, y^{\prime}}^{\prime} h_{s, y^{\prime}, y}-\sum_{y^{\prime} ; s y^{\prime}<y^{\prime}<y^{\prime} s^{\prime}} h_{s, w, y^{\prime}} h_{y^{\prime}, s^{\prime}, y}^{\prime} .
$$

If $y$ satisfies $s y<y, y s^{\prime}<y$ and either $h_{w, s^{\prime}, y}^{\prime}$ or $h_{s, w, y}$ is $\neq 0$, then $\mathbf{a}(y)>\mathbf{a}(w)$. (We certainly have $\mathbf{a}(y) \geq \mathbf{a}(w)$ by $\mathrm{P} 4$. If we had $\mathbf{a}(y)=\mathbf{a}(w)$ and $h_{s, w, y} \neq 0$ then by $\mathrm{P} 9$ we would have $y \sim_{\mathcal{L}} w$ hence $\mathcal{R}(y)=\mathcal{R}(w)$ contradicting $y s^{\prime}\left\langle y, w s^{\prime}>w\right.$. If we had $\mathbf{a}(y)=\mathbf{a}(w)$ and $h_{s, w, y}^{\prime} \neq 0$ then by P10 we would have $y \sim_{\mathcal{R}} w$ hence $\mathcal{L}(y)=\mathcal{L}(w)$, contradicting $s y<y, s w>w$.) Hence, if $s w>w, w s^{\prime}>w$, we have

$$
\left(c_{s} e_{w}\right) c_{s^{\prime}}^{\prime}-c_{s}\left(e_{w} c_{s^{\prime}}^{\prime}\right)=\sum_{y ; s y<y, y s^{\prime}<y, \mathbf{a}(y)=\mathbf{a}(w)} \alpha_{y} e_{y} \quad \bmod \tilde{\mathcal{H}}_{\geq \mathbf{a}(w)+1}
$$

We see that P15 is equivalent to the following statement.

(a) If $y, w \in W, s, s^{\prime} \in S$ are such that $s w>w, w s^{\prime}>w, s y<y, y s^{\prime}<y, \mathbf{a}(y)=$ $\mathbf{a}(w)$, then

$$
\sum_{y^{\prime} ; y^{\prime} s^{\prime}<y^{\prime}<s y^{\prime}} h_{w, s^{\prime}, y^{\prime}}^{\prime} h_{s, y^{\prime}, y}=\sum_{y^{\prime} ; s y^{\prime}<y^{\prime}<y^{\prime} s^{\prime}} h_{s, w, y^{\prime}} h_{y^{\prime}, s^{\prime}, y}^{\prime}
$$

\section{EXAMPLE: THE SPLIT CASE}

15.1. We preserve the setup of 3.1. We assume that $W, L$ is bounded, $L=l$ and that

(a) $h_{x, y, z} \in \mathbf{N}\left[v, v^{-1}\right]$ for all $x, y, z$ in $W$,

(b) $p_{y, w} \in \mathbf{N}\left[v^{-1}\right]$ for all $y, w$ in $W$.

Using an interpretation of $p_{y, w}$ in terms of intersection cohomology, as in 27.13, one can show that (a),(b) hold automatically in the split case if $W$ is integral.

Under the assumptions (a),(b) we will show that $\tilde{P}$ and P1-P3 hold for $W, l$ hence all of $\mathrm{P} 1-\mathrm{P} 14$ hold for $W, l$; we will also show that $\mathrm{P} 15$ holds.

A reference for this section is [L8].

15.2. Proof of $P 1$. The weaker inequality $\mathbf{a}(z) \leq l(z)$ for $z \in W$ was first proved in [L6] for finite $W$ and then for general $W$ by Springer (unpublished). The present argument was inspired by Springer's argument.

From 14.5(a) we see that for $x, y \in W$ we have

(a) $\sum_{z \in W} h_{x, y, z} p_{1, z} \in \mathbf{Z}\left[v^{-1}\right]$.

From 15.1(a),(b) we see that $h_{x, y, z} p_{1, z} \in \mathbf{N}\left[v, v^{-1}\right]$ for any $z \in W$. Hence in (a) there are no cancellations, so that 
(b) $h_{x, y, z} p_{1, z} \in \mathbf{N}\left[v^{-1}\right]$ for any $z \in W$.

We now fix $z \in W$ and choose $x, y \in W$ so that $\gamma_{x, y, z^{-1}} \neq 0$. From the definitions,

(c) $h_{x, y, z} p_{1, z} \in \gamma_{x, y, z^{-1}} n_{z} v^{\mathbf{a}(z)-\Delta(z)}+$ strictly smaller powers of $v$ and the coefficient of $v^{\mathbf{a}(z)-\Delta(z)}$ is $\neq 0$. Comparison with (b) gives $\mathbf{a}(z)-\Delta(z) \leq 0$.

15.3. Proof of P2. Assume that $x \neq y^{-1}$. From $14.5(\mathrm{a})$ we see that

(a) $\sum_{z \in W} h_{x, y, z} p_{1, z} \in v^{-1} \mathbf{Z}\left[v^{-1}\right]$.

As in 15.2, this implies (using 15.1(a),(b)) that

(b) $h_{x, y, z} p_{1, z} \in v^{-1} \mathbf{N}\left[v^{-1}\right]$ for any $z \in W$.

Assume now that $z=d^{-1} \in \mathcal{D}$. Then $15.2(\mathrm{c})$ becomes in our case

$$
h_{x, y, z} p_{1, z} \in \gamma_{x, y, z^{-1}} n_{z}+v^{-1} \mathbf{Z}\left[v^{-1}\right]
$$

Comparison with (b) gives $\gamma_{x, y, z^{-1}} n_{z}=0$. Since $n_{z} \neq 0$, we have $\gamma_{x, y, z^{-1}}=0$. This proves $\mathrm{P} 2$.

15.4. Proof of P3. From 14.5 (a) we see that

(a) $\sum_{z \in W} h_{y^{-1}, y, z} p_{1, z} \in 1+v^{-1} \mathbf{Z}\left[v^{-1}\right]$.

As in 15.2, this implies (using 15.1(a),(b)) that there is a unique $z$, say $z=d^{-1}$ such that

(b) $h_{y^{-1}, y, d^{-1}} p_{1, d^{-1}} \in 1+v^{-1} \mathbf{N}\left[v^{-1}\right]$

and that

(c) $h_{y^{-1}, y, z} p_{1, z} \in v^{-1} \mathbf{N}\left[v^{-1}\right]$

for all $z \neq d^{-1}$. For $z=d^{-1}, 15.2(\mathrm{c})$ becomes

$$
h_{y^{-1}, y, d^{-1}} p_{1, d^{-1}} \in \gamma_{y^{-1}, y, d} n_{d^{-1}} v^{\mathbf{a}(d)-\Delta(d)}+\text { strictly smaller powers of } v \text {. }
$$

Here $\mathbf{a}(d)-\Delta(d) \leq 0$. Comparison with (b) gives $\mathbf{a}(d)-\Delta(d)=0$ and $\gamma_{y^{-1}, y, d} n_{d^{-1}}=1$. Thus, $d \in \mathcal{D}$ and $\gamma_{y^{-1}, y, d} \neq 0$. Thus, the existence part of P3 is established.

Assume that there exists $d^{\prime} \neq d$ such that $d^{\prime} \in \mathcal{D}$ and $\gamma_{y^{-1}, y, d^{\prime}} \neq 0$. For $z=d^{\prime-1}, 15.2(\mathrm{c})$ becomes

$$
h_{y^{-1}, y, d^{\prime-1}} p_{1, d^{\prime-1}} \in \gamma_{y^{-1}, y, d^{\prime}} n_{d^{\prime-1}}+v^{-1} \mathbf{Z}\left[v^{-1}\right] .
$$

Comparison with (c) (with $z=d^{\prime-1}$ ) gives $\gamma_{y^{-1}, y, d^{\prime}} n_{d^{\prime-1}}=0$ hence $\gamma_{y^{-1}, y, d^{\prime}}=0$, a contradiction. This proves the uniqueness part of P3.

15.5. Proof of $\tilde{P}$. We may assume that $z^{\prime} \neq z$. Then we can find $s \in S$ such that $s z^{\prime}<z^{\prime}, s z>z$ and $h_{s, z, z^{\prime}} \neq 0$. Since $h_{x, y, z} \neq 0$, we have (by $\left.13.1(\mathrm{~d})\right) z \leq_{\mathcal{R}} x$ hence $\mathcal{L}(x) \subset \mathcal{L}(z)$ (by 8.6). Since $s \notin \mathcal{L}(z)$, we have $s \notin \mathcal{L}(x)$, that is, $s x>x$. We have $c_{s} c_{x} c_{y}=\sum_{u} p_{u} c_{u}$, where

$$
p_{u}=\sum_{w} h_{x, y, w} h_{s, w, u}=\sum_{x^{\prime}} h_{s, x, x^{\prime}} h_{x^{\prime}, y, u}
$$


In particular,

$$
p_{z^{\prime}}=\sum_{w} h_{x, y, w} h_{s, w, z^{\prime}}=h_{x, y, z} h_{s, z, z^{\prime}}+\sum_{w ; w \neq z} h_{x, y, w} h_{s, w, z^{\prime}}
$$

By 6.5, we have $h_{s, z, z^{\prime}} \in \mathbf{Z}$ hence

$$
\pi_{n}\left(p_{z^{\prime}}\right)=\pi_{n}\left(h_{x, y, z}\right) h_{s, z, z^{\prime}}+\sum_{w ; w \neq z} \pi_{n}\left(h_{x, y, w} h_{s, w, z^{\prime}}\right)
$$

for any $n \in \mathbf{Z}$. In particular, this holds for $n=\mathbf{a}(z)$. By assumption, we have $\pi_{n}\left(h_{x, y, z}\right) \neq 0$ and $h_{s, z, z^{\prime}} \neq 0$; hence, by 15.1(a), we have $\pi_{n}\left(h_{x, y, z}\right)>0$ and $h_{s, z, z^{\prime}}>0$. Again, by 15.1(a) we have $\pi_{n}\left(h_{x, y, w} h_{s, w, z^{\prime}}\right) \geq 0$ for any $w \neq z$. Hence from (a) we deduce $\pi_{n}\left(p_{z^{\prime}}\right)>0$. Since $p_{z^{\prime}}=\sum_{x^{\prime}} h_{s, x, x^{\prime}} h_{x^{\prime}, y, z^{\prime}}$, there exists $x^{\prime}$ such that $\pi_{n}\left(h_{s, x, x^{\prime}} h_{x^{\prime}, y, z^{\prime}}\right) \neq 0$. Since $s x>x$, we see from 6.5 that $h_{s, x, x^{\prime}} \in \mathbf{Z}$ hence

$$
\pi_{n}\left(h_{s, x, x^{\prime}} h_{x^{\prime}, y, z^{\prime}}\right)=h_{s, x, x^{\prime}} \pi_{n}\left(h_{x^{\prime}, y, z^{\prime}}\right) .
$$

Thus we have $\pi_{n}\left(h_{x^{\prime}, y, z^{\prime}}\right) \neq 0$. This proves $\tilde{P}$ in our case.

15.6. Since $\tilde{P}$ and $\mathrm{P} 1-\mathrm{P} 3$ are known, we see that $\mathrm{P} 1-\mathrm{P} 11$ and $\mathrm{P} 13, \mathrm{P} 14$ hold in our case (see $\S 14$ ). The same arguments can be applied to $W_{I}$ where $I \subset S$, hence P1-P11 and P13,P14 hold for $W_{I}$. By 14.12, P12 holds for $W$. Thus, P1-P14 hold for $W$.

15.7. Proof of P15. By 14.15, we see that it is enough to prove 14.15(a). Let $y, w, s, s^{\prime}$ be as in 14.15(a). In our case, by 6.5, the equation in 14.15(a) involves only integers, hence it is enough to prove it after specializing $v=v^{\prime}$. If in 14.15 we specialize $v=v^{\prime}$, then the left and right module structures in 14.15 clearly commute, since the left and right regular representations of $\mathcal{H}$ commute. Hence the coefficient of $e_{y}$ in $\left(\left(c_{s} e_{w}\right) c_{s^{\prime}}-c_{s}\left(e_{w} c_{s^{\prime}}\right)\right)_{v=v^{\prime}}$ is 0 . By the computation in 14.15 , this coefficient is

(a)

$$
\left(h_{w, s^{\prime}, y}-h_{s, w, y}\right)\left(v+v^{-1}\right)+\sum_{\substack{y^{\prime} \\ y^{\prime} s^{\prime}<y^{\prime}<s y^{\prime}}} h_{w, s^{\prime}, y^{\prime}} h_{s, y^{\prime}, y}-\sum_{\substack{y^{\prime} \\ s y^{\prime}<y^{\prime}<y^{\prime} s^{\prime}}} h_{s, w, y^{\prime}} h_{y^{\prime}, s^{\prime}, y}=0 .
$$

By $6.5, h_{s, w, y}$ is the coefficient of $v^{-1}$ in $p_{y, w}$ and $h_{w, s^{\prime}, y}=h_{s^{\prime}, w^{-1}, y^{-1}}$ is the coefficient of $v^{-1}$ in $p_{y^{-1}, w^{-1}}=p_{y, w}$. Thus, $h_{s, w, y}=h_{w, s^{\prime}, y}$ and (a) reduces to the equation in 14.15(a) (specialized at $v=v^{\prime}$ ). This proves 14.15(a).

\section{EXAMPLE: THE QUASISPlit CASE}

16.1. Let $G, F$ be as in 0.2 . Let $(\tilde{W}, \tilde{S})$ be the Weyl group of $G$ and let $u: \tilde{W} \rightarrow \tilde{W}$ be the automorphism of $\tilde{W}$ induced by $F$; this restricts to a permutation of $\tilde{S}$. Let $\tilde{W}^{u}=\{w \in \tilde{W} ; u(w)=w\}$. For any $u$-orbit o on $\tilde{S}$, we set $s_{\mathbf{o}}=w_{0}^{\mathbf{o}} \in \tilde{W}^{u}$. The elements $s_{\mathbf{o}}$ for various o as above form a subset $\tilde{S}_{u}$ of $\tilde{W}^{u}$. 
Lemma 16.2. $\left(\tilde{W}^{u}, \tilde{S}_{u}\right)$ is a Coxeter group and the restriction to $\tilde{W}^{u}$ of the length function $\tilde{l}: \tilde{W} \rightarrow \mathbf{N}$ is a weight function $L: \tilde{W}^{u} \rightarrow \mathbf{N}$.

We omit the proof.

16.3. In this section we assume that $(W, S)$ is the Coxeter group $\left(\tilde{W}^{u}, \tilde{S}_{u}\right)$ and that $L: W \rightarrow \mathbf{N}$ is as in 16.2. We then say that we are in the quasisplit case.

We denote $h_{x, y, z}, p_{x, y}, \mathbf{a}(z), \gamma_{x, y, z}, \Delta(z), \mathcal{D}$ defined in terms of $\tilde{W}, \tilde{l}$ by

$$
\tilde{h}_{x, y, z}, \tilde{p}_{x, y}, \tilde{\mathbf{a}}(z), \tilde{\gamma}_{x, y, z}, \tilde{\Delta}(z), \tilde{\mathcal{D}} .
$$

One can show using the geometric interpretation in 27.13 that (a),(b) below hold.

(a) For any $x, y, z \in \tilde{W}$ and any integer $n$ there exists a $\mathbf{Q}$-vector space $V_{x, y, z}^{n}$ such that $\tilde{h}_{x, y, z}=\sum_{n} \operatorname{dim} V_{x, y, z}^{n} v^{n} ;$ for any $x, y \in \tilde{W}$ and any integer $n \leq 0$ there exists a Q-vector space $V_{x, y}^{n}$ such that $\tilde{p}_{x, y}=\sum_{n \leq 0} \operatorname{dim} V_{x, y}^{n} v^{n}$.

(In other words, $(\tilde{W}, \tilde{l})$ satisfies the requirements of 15.1.)

(b) For any $x, y, z \in W$ and any integer $n, V_{x, y, z}^{n}$ carries a linear transformation $u$ of finite order and $h_{x, y, z}=\sum_{n} \operatorname{tr}\left(u, V_{x, y, z}^{n}\right) v^{n} ;$ for any $x, y \in W$ and any integer $n \leq 0, V_{x, y}^{n}$ carries a linear transformation $u$ of finite order and $p_{x, y}=\sum_{n} \operatorname{tr}\left(u, V_{x, y}^{n}\right) v^{n}$.

We deduce that (c)-(f) below hold:

(c) If $x, y, z \in W, n \in \mathbf{Z}$ and $\pi_{n}\left(h_{x, y, z}\right) \neq 0$ then $\pi_{n}\left(\tilde{h}_{x, y, z}\right) \neq 0$.

(d) If $x, y \in W, n \in \mathbf{Z}$ and $\pi_{n}\left(p_{x, y}\right) \neq 0$ then $\pi_{n}\left(\tilde{p}_{x, y}\right) \neq 0$.

(e) If $x, y, z \in W, n \in \mathbf{Z}$ and $\pi_{n}\left(\tilde{h}_{x, y, z}\right)= \pm 1$ then $\pi_{n}\left(h_{x, y, z}\right)= \pm 1$.

(f) If $x, y \in W, n \in \mathbf{Z}$ and $\pi_{n}\left(\tilde{p}_{x, y}\right)= \pm 1$ then $\pi_{n}\left(p_{x, y}\right)= \pm 1$.

16.4. By our assumptions, the results of $\S 15$ are applicable to $\tilde{W}, \tilde{l}$. Under the assumptions above, we will show that P1-P14 hold for $W, L$.

Lemma 16.5. For $z \in W$ we have $\mathbf{a}(z)=\tilde{\mathbf{a}}(z)$ and $\tilde{\Delta}(z) \leq \Delta(z)$.

We can find $x, y \in W$ such that $\pi_{\mathbf{a}(z)}\left(h_{x, y, z}\right) \neq 0$. By 16.3(c) we have $\pi_{\mathbf{a}(z)}\left(\tilde{h}_{x, y, z}\right) \neq 0$. Hence $\mathbf{a}(z) \leq \tilde{\mathbf{a}}(z)$. By P3,P5 for $\tilde{W}$, there is a unique $d \in \tilde{\mathcal{D}}$ such that $\tilde{\gamma}_{z^{-1}, z, d}= \pm 1$. The uniqueness of $d$ implies that $d$ is fixed by $u$. Thus $d \in W$. By $\mathrm{P} 7$ for $\tilde{W}$, we have $\tilde{\gamma}_{z, d, z^{-1}}= \pm 1$. Hence $\pi_{\tilde{\mathbf{a}}(z)}\left(\tilde{h}_{z, d, z}\right)= \pm 1$. By $16.3(\mathrm{e})$, we have $\pi_{\tilde{\mathbf{a}}(z)}\left(h_{z, d, z}\right)= \pm 1$. Hence $\tilde{\mathbf{a}}(z) \leq \mathbf{a}(z)$ so that $\tilde{\mathbf{a}}(z)=\mathbf{a}(z)$.

By definition, we have $\pi_{-\Delta(z)}\left(p_{1, z}\right) \neq 0$. Using 16.3(d), we deduce that $\pi_{-\Delta(z)}\left(\tilde{p}_{1, z}\right) \neq 0$. Hence $-\Delta(z) \leq-\tilde{\Delta}(z)$. The lemma is proved.

Lemma 16.6. $\mathcal{D}=\tilde{\mathcal{D}} \cap W$.

Let $d \in \mathcal{D}$. We have $\mathbf{a}(d)=\Delta(d)$. Using 16.5, we deduce $\tilde{\mathbf{a}}(d)=\Delta(d)$. By $\mathrm{P} 1$ for $\tilde{W}$, we have $\tilde{\mathbf{a}}(d) \leq \tilde{\Delta}(d)$. Hence $\Delta(d) \leq \tilde{\Delta}(d)$. Using 16.5 , we deduce $\Delta(d)=\tilde{\Delta}(d)$ so that $\tilde{\Delta}(d)=\tilde{\mathbf{a}}(d)$ and $d \in \tilde{\mathcal{D}}$.

Conversely, let $d \in \tilde{\mathcal{D}} \cap W$. We have $\tilde{\mathbf{a}}(d)=\tilde{\Delta}(d)$. Using 16.5 we deduce $\mathbf{a}(d)=\tilde{\Delta}(d)$. By P5 for $\tilde{W}$, we have $\pi_{-\tilde{\Delta}(d)}\left(\tilde{p}_{1, d}\right)= \pm 1$. Using $16.3(\mathrm{f})$ we deduce 
$\pi_{-\tilde{\Delta}(d)}\left(p_{1, d}\right)= \pm 1$. Hence $-\tilde{\Delta}(d) \leq-\Delta(d)$. Using 16.5 we deduce $\Delta(d)=\tilde{\Delta}(d)$ so that $\Delta(d)=\mathbf{a}(d)$ and $d \in \mathcal{D}$. The lemma is proved.

Lemma 16.7. (a) Let $x, y, z \in W$ be such that $\gamma_{x, y, z} \neq 0$. Then $\tilde{\gamma}_{x, y, z} \neq 0$.

(b) Let $x, y, z \in W$ be such that $\tilde{\gamma}_{x, y, z}= \pm 1$. Then $\gamma_{x, y, z}= \pm 1$.

In the setup of (a) we have $\pi_{\mathbf{a}\left(z^{-1}\right)}\left(h_{x, y, z^{-1}}\right) \neq 0$. Using 16.5 we deduce that $\pi_{\tilde{\mathbf{a}}\left(z^{-1}\right)}\left(h_{x, y, z^{-1}}\right) \neq 0$. Using $16.3(\mathrm{c})$, we deduce that $\pi_{\tilde{\mathbf{a}}\left(z^{-1}\right)}\left(\tilde{h}_{x, y, z^{-1}}\right) \neq 0$. Hence $\tilde{\gamma}_{x, y, z} \neq 0$.

In the setup of (b) we have $\pi_{\tilde{\mathbf{a}}\left(z^{-1}\right)}\left(\tilde{h}_{x, y, z^{-1}}\right)= \pm 1$. Using 16.5 , we deduce $\pi_{\mathbf{a}\left(z^{-1}\right)}\left(\tilde{h}_{x, y, z^{-1}}\right)= \pm 1$. Using $16.3(\mathrm{e})$, we deduce $\pi_{\mathbf{a}\left(z^{-1}\right)}\left(h_{x, y, z^{-1}}\right)= \pm 1$. Hence $\gamma_{x, y, z}= \pm 1$.

16.8. Proof of P1. By 16.5 and $\mathrm{P} 1$ for $\tilde{W}$, we have $\mathbf{a}(z)=\tilde{\mathbf{a}}(z) \leq \tilde{\Delta}(z) \leq \Delta(z)$, hence $\mathbf{a}(z) \leq \Delta(z)$.

16.9. Proof of $\mathrm{P} 2$. In the setup of $\mathrm{P} 2$, we have (by 16.7) $\tilde{\gamma}_{x, y, d} \neq 0$ and $d \in \tilde{\mathcal{D}}$ (see 16.6). Using P2 for $\tilde{W}$, we deduce $x=y^{-1}$.

16.10. Proof of P3. Let $y \in W$. By P3 for $\tilde{W}$, there is a unique $d \in \tilde{\mathcal{D}}$ such that $\tilde{\gamma}_{y^{-1}, y, d} \neq 0$. By the uniqueness of $d$, we have $u(d)=d$ hence $d \in W$. Using P5 for $\tilde{W}$, we see that $\tilde{\gamma}_{y^{-1}, y, d}= \pm 1$. Using 16.7, we deduce $\gamma_{y^{-1}, y, d}= \pm 1$. Since $d \in \mathcal{D}$ by 16.6 , the existence part of P3 is established. Assume now that $d^{\prime} \in \mathcal{D}$ satisfies $\gamma_{y^{-1}, y, d^{\prime}} \neq 0$. Using 16.7 , we deduce $\tilde{\gamma}_{y^{-1}, y, d^{\prime}} \neq 0$. Since $d^{\prime} \in \tilde{\mathcal{D}}$ by 16.6 , we can use the uniqueness in P3 for $\tilde{W}$ to deduce that $d=d^{\prime}$. Thus P3 holds for $W$.

16.11. Proof of $P 4$. We may assume that there exists $s \in S$ such that $h_{s, z, z^{\prime}} \neq 0$ or $h_{z, s, z^{\prime}} \neq 0$. In the first case, using $16.3(\mathrm{c})$, we deduce $\tilde{h}_{s, z, z^{\prime}} \neq 0$. Hence $z^{\prime} \leq_{\mathcal{L}} z$ (in $\tilde{W}$ ) and using $\mathrm{P} 4$ for $\tilde{W}$, we deduce that $\tilde{\mathbf{a}}\left(z^{\prime}\right) \geq \tilde{\mathbf{a}}(z)$. Using now 16.5 , we see that $\mathbf{a}\left(z^{\prime}\right) \geq \mathbf{a}(z)$. The proof in the second case is entirely similar.

16.12. Now $\mathrm{P} 5$ is proved as in 14.5; $\mathrm{P} 6$ is proved as in 14.6; $\mathrm{P} 7$ is proved as in 14.7; P8 is proved as in 14.8; $\mathrm{P} 12$ is proved as in 14.12 .

16.13. Proof of P13. If $z^{\prime} \leftarrow \mathcal{L} z$ in $W$, then there exists $s \in S$ such that $h_{s, z, z^{\prime}} \neq 0$ hence, by $16.3(\mathrm{c}), \tilde{h}_{s, z, z^{\prime}} \neq 0$, hence $z^{\prime} \leq_{\mathcal{L}} z$ in $\tilde{W}$. It follows that

(a) $z^{\prime} \leq_{\mathcal{L}} z($ in $W)$ implies $z^{\prime} \leq_{\mathcal{L}} z($ in $\tilde{W})$.

Hence

(b) $z^{\prime} \sim_{\mathcal{L}} z($ in $W)$ implies $z^{\prime} \sim_{\mathcal{L}} z($ in $\tilde{W})$.

Thus any left cell of $W$ is contained in a left cell of $\tilde{W}$.

In the setup of P13, let $\tilde{\Gamma}$ be the left cell of $\tilde{W}$ containing $\Gamma$. Let $x \in \Gamma$. By P3 for $W$, there exists $d \in \mathcal{D}$ such that $\gamma_{x^{-1}, x, d} \neq 0$. By P8 for $W$, we have $x \sim_{\mathcal{L}} d^{-1}$ hence $d^{-1} \in \Gamma$. Using $\mathrm{P} 6$ we have $d=d^{-1}$, hence $d \in \Gamma$. It remains to prove the uniqueness of $d$. Let $d^{\prime}, d^{\prime \prime}$ be elements of $\mathcal{D} \cap \Gamma$. We must prove that $d^{\prime}=d^{\prime \prime}$. Now $d^{\prime}, d^{\prime \prime}$ belong to $\tilde{\Gamma}$ and, by 16.6 , are in $\tilde{\mathcal{D}}$. Using $\mathrm{P} 13$ for $\tilde{W}$, it follows that $d^{\prime}=d^{\prime \prime}$. Thus P13 holds for $W$. 
Lemma 16.14. Let $x, y \in W$. We have $x \sim_{\mathcal{L}} y$ (in $W$ ) if and only if $x \sim_{\mathcal{L}} y$ (in $\tilde{W})$.

If $x \sim_{\mathcal{L}} y($ in $W)$ then $x \sim_{\mathcal{L}} y($ in $\tilde{W})$, by $16.13(\mathrm{~b})$.

Assume now that $x \sim_{\mathcal{L}} y($ in $\tilde{W})$. Let $d, d^{\prime} \in \mathcal{D}$ be such that $x \sim_{\mathcal{L}} d($ in $W$ ) and $y \sim_{\mathcal{L}} d^{\prime}$ (in $W$ ); see P13. By the first line of the proof we have $x \sim_{\mathcal{L}} d$ (in $\tilde{W}$ ) and $y \sim_{\mathcal{L}} d^{\prime}($ in $\tilde{W})$. Hence $d \sim_{\mathcal{L}} d^{\prime}$ (in $\tilde{W}$ ). Since $d, d^{\prime} \in \tilde{\mathcal{D}}$, we deduce (using P13 for $\tilde{W}$ ) that $d=d^{\prime}$. It follows that $x \sim_{\mathcal{L}} y$ (in $W$ ). The lemma is proved.

16.15. Proof of P9. We assume that $z^{\prime} \leq_{\mathcal{L}} z$ (in $W$ ) and $\mathbf{a}\left(z^{\prime}\right)=\mathbf{a}(z)$. By 16.13(a), it follows that $z^{\prime} \leq_{\mathcal{L}} z$ (in $\tilde{W}$ ) and, using 16.5, that $\tilde{\mathbf{a}}\left(z^{\prime}\right)=\tilde{\mathbf{a}}(z)$. Using now P9 in $\tilde{W}$, it follows that $z^{\prime} \sim_{\mathcal{L}} z($ in $\tilde{W})$. Using 16.14 , we deduce that $z^{\prime} \sim_{\mathcal{L}} z$ (in $W$ ).

16.16. Now $\mathrm{P} 10$ is proved as in 14.10 ; $\mathrm{P} 11$ is proved as in 14.11 ; $\mathrm{P} 14$ is proved as in 14.14 .

16.17. We sketch a proof of $\mathrm{P} 15$ in our case.

A refinement of the proof of $\mathrm{P} 15$ given in $14.15,15.7$ provides, for any $w, y, x, x^{\prime}$ in $\tilde{W}$ and any $k$, an isomorphism of vector spaces

$$
\oplus_{j+j^{\prime}=k} \oplus_{y^{\prime} \in \tilde{W}} V_{w, x^{\prime}, y^{\prime}}^{j^{\prime}} \otimes V_{x, y^{\prime}, y}^{j} \stackrel{\sim}{\rightarrow} \oplus_{j+j^{\prime}=k} \oplus_{y^{\prime} \in \tilde{W}} V_{x, w, y^{\prime}}^{j} \otimes V_{y^{\prime}, x^{\prime}, y}^{j^{\prime}} .
$$

which (assuming that $\tilde{\mathbf{a}}(w)=\tilde{\mathbf{a}}(y)$ ) restricts to an isomorphism

$$
\oplus_{y^{\prime} \in \tilde{W}} V_{w, x^{\prime}, y^{\prime}}^{j^{\prime}} \otimes V_{x, y^{\prime}, y}^{j} \stackrel{\sim}{\longrightarrow} \oplus_{y^{\prime} \in \tilde{W}} V_{x, w, y^{\prime}}^{j} \otimes V_{y^{\prime}, x^{\prime}, y}^{j^{\prime}}
$$

for any $j, j^{\prime}$ such that $j+j^{\prime}=k$.

Assuming now that $w, y, x, x^{\prime} \in W$, we can take traces of $u$ in both sides; we deduce

$$
\sum_{y^{\prime} \in W} \pi_{j^{\prime}}\left(h_{w, x^{\prime}, y^{\prime}}\right) \pi_{j}\left(h_{x, y^{\prime}, y}\right)=\sum_{y^{\prime} \in W} \pi_{j}\left(h_{x, w, y^{\prime}}\right) \pi_{j^{\prime}}\left(h_{y^{\prime}, x^{\prime}, y}\right)
$$

(the summands corresponding to $y^{\prime} \in \tilde{W}-W$ do not contribute to the trace) or equivalently

$$
\sum_{y^{\prime} \in W} h_{w, x^{\prime}, y^{\prime}}^{\prime} h_{x, y^{\prime}, y}=\sum_{y^{\prime} \in W} h_{x, w, y^{\prime}} h_{y^{\prime}, x^{\prime}, y}^{\prime}
$$

as required.

16.18. Everything in $16.1-16.17$ extends to the case where $G, F$ is replaced by $\mathbf{G}, F$ as in 0.6 .

\section{EXAMPLE: THE INFINITE DIHEDRAL CASE}

17.1. In this section we preserve the setup of 7.1. We assume that $m=\infty$ and that $L_{2}>L_{1}$. We will show that P1-P15 hold in this case.

Let $\zeta=v^{L_{2}-L_{1}}+v^{L_{1}-L_{2}}$. For $a \in\{1,2\}$, let $f_{a}=v^{L_{a}}+v^{-L_{a}}$. For $m, n \in \mathbf{Z}$ we define $\delta_{m<n}$ to be 1 if $m<n$ and to be 0 otherwise. 
17.2. From 7.5, 7.6 we have for all $k^{\prime} \in \mathbf{N}$ :

$$
\begin{aligned}
& c_{1} c_{2_{k^{\prime}}}=c_{1_{k^{\prime}+1}}, \\
& c_{2} c_{1_{k^{\prime}}}=c_{2_{k^{\prime}+1}}+\delta_{k^{\prime}>1} \zeta c_{2_{k^{\prime}-1}}+\delta_{k^{\prime}>3} c_{2_{k^{\prime}-3}} .
\end{aligned}
$$

Proposition 17.3. For $k \geq 0, k^{\prime} \geq 1$ we have

(a) $c_{2 k+1} c_{2_{k^{\prime}}}=f_{2} \sum_{u \in[0, k] ; 2 u \leq k^{\prime}-1} c_{2_{2 k+k^{\prime}-4 u}}$,

(b) $c_{1_{2 k+2}} c_{k_{k^{\prime}}}=f_{2} \sum_{u \in[0, k] ; 2 u \leq k^{\prime}-1} c_{1_{2 k+k^{\prime}+1-4 u}}$.

Assume that $k=0$. Using 17.2 we have $c_{2} c_{2_{k^{\prime}}}=f_{2} c_{2_{k^{\prime}}}$.

Assume now that $k=1$. Using 17.2 , we have $c_{2_{3}}=c_{2} c_{1} c_{2}-\zeta c_{2}$. Using this and 17.2 , we have

$$
\begin{aligned}
& c_{23} c_{k_{k^{\prime}}}=c_{2} c_{1} c_{2} c_{2_{k^{\prime}}}-\zeta c_{2} c_{2_{k^{\prime}}}=f_{2} c_{2} c_{k_{k^{\prime}+1}}-f_{2} \zeta c_{k_{k^{\prime}}} \\
& =f_{2} c_{2_{k^{\prime}+2}}+f_{2} \zeta c_{2_{k^{\prime}}}+\delta_{k^{\prime}>2} f_{2} c_{2_{k^{\prime}-2}}-f_{2} \zeta c_{k_{k^{\prime}}}=f_{2} c_{2_{k^{\prime}+2}}+\delta_{k^{\prime}>2} f_{2} c_{2_{k^{\prime}-2}},
\end{aligned}
$$

as required. We prove the equality in (a) for fixed $k^{\prime}$, by induction on $k$. The cases $k=0,1$ are already known. If $k=2$ then using 17.2 , we have $c_{2_{5}}=$ $c_{2} c_{1} c_{2_{3}}-\zeta c_{2_{3}}-c_{2_{1}}$. Using this, 17.2 , and the induction hypothesis, we have

$$
\begin{aligned}
& c_{25} c_{2_{k^{\prime}}}=c_{2} c_{1} c_{23} c_{k_{k^{\prime}}}-\zeta c_{23} c_{k_{k^{\prime}}}-c_{2} c_{k_{k^{\prime}}} \\
& =f_{2} c_{2} c_{1} c_{2_{k^{\prime}+2}}+\delta_{k^{\prime}>2} f_{2} c_{2} c_{1} c_{k_{k^{\prime}-2}}-\zeta f_{2} c_{2_{k^{\prime}+2}}-\delta_{k^{\prime}>2} \zeta f_{2} c_{2_{k^{\prime}-2}}-f_{2} c_{2_{k^{\prime}}} \\
& =f_{2} c_{2} c_{1_{k^{\prime}+3}}+\delta_{k^{\prime}>2} f_{2} c_{2} c_{1_{k^{\prime}-1}}-\zeta f_{2} c_{2_{k^{\prime}+2}}-\delta_{k^{\prime}>2} \zeta f_{2} c_{k_{k^{\prime}-2}}-f_{2} c_{2_{k^{\prime}}} \\
& =f_{2} c_{k_{k^{\prime}+4}}+f_{2} \zeta c_{2_{k^{\prime}+2}}+f_{2} c_{2_{k^{\prime}}}+\delta_{k^{\prime}>2} f_{2} c_{2_{k^{\prime}}}+\delta_{k^{\prime}>2} f_{2} \zeta c_{2_{k^{\prime}-2}}+\delta_{k^{\prime}>4} f_{2} c_{k_{k^{\prime}-4}} \\
& -\zeta f_{2} c_{2_{k^{\prime}+2}}-\delta_{k^{\prime}>2} \zeta f_{2} c_{2_{k^{\prime}-2}}-f_{2} c_{2_{k^{\prime}}}=f_{2} c_{k_{k^{\prime}+4}}+\delta_{k^{\prime}>2} f_{2} c_{2_{k^{\prime}}}+\delta_{k^{\prime}>4} f_{2} c_{k_{k^{\prime}-4}},
\end{aligned}
$$

as required. A similar argument applies for $k \geq 3$. This proves (a).

(b) is obtained by multiplying both sides of (a) by $c_{1}$ on the left. The proposition is proved.

Proposition 17.4. For $k \geq 0, k^{\prime} \geq 1$, we have

(a) $c_{2_{2 k+1}} c_{1_{k^{\prime}}}=\sum_{u \in[0,2 k+2]} p_{u} c_{2_{k^{\prime}+2 k+1-2 u}}$,

(b) $c_{1_{2 k+2}} c_{1_{k^{\prime}}}=\sum_{u \in[0,2 k+2]} p_{u} c_{1_{k^{\prime}+2 k+2-2 u}}$,

(c) $c_{1_{k^{\prime}}^{-1}} c_{2 k+1}=\sum_{u \in[0,2 k+2]} p_{u} c_{2_{k^{\prime}+2 k+1-2 u}^{-1}}$,

(d) $c_{1_{k^{\prime}}^{-1}} c_{1_{2 k+2}^{-1}}=\sum_{u \in[0,2 k+2]} p_{u} c_{1_{k^{\prime}+2 k+1-2 u}^{-1}}$,

(e) $c_{2 k+2} c_{k_{k^{\prime}}}=\sum_{u \in[0,2 k+2]} f_{1} p_{u} c_{k_{k^{\prime}+2 k+1-2 u}}$,

(f) $c_{1_{2 k+3}} c_{k_{k^{\prime}}}=\sum_{u \in[0,2 k+2]} f_{1} p_{u} c_{1_{k^{\prime}+2 k+2-2 u}}$,

(g) $c_{1_{1}} c_{1_{k^{\prime}}}=f_{1} c_{1_{k^{\prime}}}$

where

$$
\begin{aligned}
& p_{0}=1, p_{2 k+2}=\delta_{k^{\prime}>2 k+3}, \\
& p_{u}=\delta_{k^{\prime}>u} \zeta \text { for } u=1,3,5, \ldots, 2 k+1, \\
& p_{u}=\delta_{k^{\prime}>u-1}+\delta_{k^{\prime}>u+1} \text { for } u=2,4,6, \ldots, 2 k .
\end{aligned}
$$

We prove (a). For $k=0$ the equality in (a) is $c_{2} c_{1_{k^{\prime}}}=c_{2_{k^{\prime}+1}}+\delta_{k^{\prime}>1} \zeta c_{2_{k^{\prime}-1}}+$ $\delta_{k^{\prime}>3} c_{k_{k^{\prime}-3}}$ which is contained in 17.2. Assume now that $k=1$. Using $c_{2_{3}}=$ 
$c_{2} c_{1} c_{2}-\zeta c_{2}$ and 17.2 , we have

$$
\begin{aligned}
& c_{23} c_{k_{k^{\prime}}}=c_{2} c_{1} c_{2} c_{k_{k^{\prime}}}-\zeta c_{2} c_{k_{k^{\prime}}}=c_{2} c_{1} c_{2_{k^{\prime}+1}}+\delta_{k^{\prime}>1} \zeta c_{2} c_{1} c_{k_{k^{\prime}-1}}+\delta_{k^{\prime}>3} c_{2} c_{1} c_{2_{k^{\prime}-3}} \\
& -\zeta c_{k_{k^{\prime}+1}}-\delta_{k^{\prime}>1} \zeta^{2} c_{2_{k^{\prime}-1}}-\delta_{k^{\prime}>3} \zeta c_{2_{k^{\prime}-3}}=c_{2} c_{1_{k^{\prime}+2}}+\delta_{k^{\prime}>1} \zeta c_{2} c_{1_{k^{\prime}}} \\
& +\delta_{k^{\prime}>3} c_{2} c_{1_{k^{\prime}-2}}-\zeta c_{k_{k^{\prime}+1}}-\delta_{k^{\prime}>1} \zeta^{2} c_{2_{k^{\prime}-1}}-\delta_{k^{\prime}>3} \zeta c_{k_{k^{\prime}-3}} \\
& =c_{2_{k^{\prime}+3}}+\zeta c_{k_{k^{\prime}+1}}+\delta_{k^{\prime}>1} c_{k_{k^{\prime}-1}}+\delta_{k^{\prime}>1} \zeta c_{2_{k^{\prime}+1}}+\delta_{k^{\prime}>1} \zeta^{2} c_{2_{k^{\prime}-1}}+\delta_{k^{\prime}>3} \zeta c_{2_{k^{\prime}-3}} \\
& +\delta_{k^{\prime}>3} c_{k_{k^{\prime}-1}}+\delta_{k^{\prime}>3} \zeta c_{k_{k^{\prime}-3}}+\delta_{k^{\prime}>5} c_{2_{k^{\prime}-5}}-\zeta c_{k_{k^{\prime}+1}}-\delta_{k^{\prime}>1} \zeta^{2} c_{k_{k^{\prime}-1}}-\delta_{k^{\prime}>3} \zeta c_{k_{k^{\prime}-3}} \\
& =c_{2_{k^{\prime}+3}}+\delta_{k^{\prime}>1} \zeta c_{k_{k^{\prime}+1}}+\left(\delta_{k^{\prime}>1}+\delta_{k^{\prime}>3}\right) c_{2_{k^{\prime}-1}}+\delta_{k^{\prime}>3} \zeta c_{2_{k^{\prime}-3}}+\delta_{k^{\prime}>5} c_{2_{k^{\prime}-5}},
\end{aligned}
$$

as required.

We prove the equality in (a) for fixed $k^{\prime}$, by induction on $k$. The cases $k=0,1$ are already known. Assume now that $k=2$. Using $c_{2_{5}}=c_{2} c_{1} c_{2_{3}}-\zeta c_{2_{3}}-c_{2_{1}}$, 17.2 , and the case $k=1$, we have

$$
\begin{aligned}
& c_{2_{5}} c_{1_{k^{\prime}}}=c_{2} c_{1} c_{2_{3}} c_{1_{k^{\prime}}}-\zeta c_{2_{3}} c_{1_{k^{\prime}}}-c_{2_{1}} c_{1_{k^{\prime}}} \\
& =c_{2} c_{1} c_{k_{k^{\prime}+3}}+\delta_{k^{\prime}>1} \zeta c_{2} c_{1} c_{k_{k^{\prime}+1}}+\left(\delta_{k^{\prime}>1}+\delta_{k^{\prime}>3}\right) c_{2} c_{1} c_{2_{k^{\prime}-1}}+\delta_{k^{\prime}>3} \zeta c_{2} c_{1} c_{2_{k^{\prime}-3}} \\
& +\delta_{k^{\prime}>5} c_{2} c_{1} c_{k_{k^{\prime}-5}}-\zeta c_{k_{k^{\prime}+3}}-\delta_{k^{\prime}>1} \zeta^{2} c_{2_{k^{\prime}+1}}-\left(\delta_{k^{\prime}>1}+\delta_{k^{\prime}>3}\right) \zeta c_{k_{k^{\prime}-1}} \\
& -\delta_{k^{\prime}>3} \zeta^{2} c_{2_{k^{\prime}-3}}-\delta_{k^{\prime}>5} \zeta c_{2_{k^{\prime}-5}}-c_{2_{k^{\prime}+1}}-\delta_{k^{\prime}>1} \zeta c_{2_{k^{\prime}-1}}-\delta_{k^{\prime}>3} c_{2_{k^{\prime}-3}} \\
& =c_{2} c_{k_{k^{\prime}+4}}+\delta_{k^{\prime}>1} \zeta c_{2} c_{k_{k^{\prime}+2}}+\left(\delta_{k^{\prime}>1}+\delta_{k^{\prime}>3}\right) c_{2} c_{k_{k^{\prime}}}+\delta_{k^{\prime}>3} \zeta c_{2} c_{k_{k^{\prime}-2}} \\
& +\delta_{k^{\prime}>5} c_{2} c_{k_{k^{\prime}-4}}-\zeta c_{k_{k^{\prime}+3}}-\delta_{k^{\prime}>1} \zeta^{2} c_{k_{k^{\prime}+1}}-\left(\delta_{k^{\prime}>1}+\delta_{k^{\prime}>3}\right) \zeta c_{2_{k^{\prime}-1}} \\
& -\delta_{k^{\prime}>3} \zeta^{2} c_{2_{k^{\prime}-3}}-\delta_{k^{\prime}>5} \zeta c_{2_{k^{\prime}-5}}-c_{2_{k^{\prime}+1}}-\delta_{k^{\prime}>1} \zeta c_{2_{k^{\prime}-1}}-\delta_{k^{\prime}>3} c_{2_{k^{\prime}-3}} \\
& =c_{k_{k^{\prime}+5}}+\zeta c_{2_{k^{\prime}+3}}+c_{2_{k^{\prime}+1}}+\delta_{k^{\prime}>1} \zeta c_{2_{k^{\prime}+3}}+\delta_{k^{\prime}>1} \zeta^{2} c_{2_{k^{\prime}+1}}+\delta_{k^{\prime}>1} \zeta c_{2_{k^{\prime}-1}} \\
& +\left(\delta_{k^{\prime}>1}+\delta_{k^{\prime}>3}\right) c_{2_{k^{\prime}+1}}+\left(\delta_{k^{\prime}>1}+\delta_{k^{\prime}>3}\right) \zeta c_{k_{k^{\prime}-1}}+2 \delta_{k^{\prime}>3} c_{2_{k^{\prime}-3}}+\delta_{k^{\prime}>3} \zeta c_{2_{k^{\prime}-1}} \\
& +\delta_{k^{\prime}>3} \zeta^{2} c_{k_{k^{\prime}-3}}+\delta_{k^{\prime}>5} \zeta c_{2_{k^{\prime}-5}}+\delta_{k^{\prime}>5} c_{2_{k^{\prime}-3}}+\delta_{k^{\prime}>5} \zeta c_{2_{k^{\prime}-5}}+\delta_{k^{\prime}>7} c_{2_{k^{\prime}-7}} \\
& -\zeta c_{k_{k^{\prime}+3}}-\delta_{k^{\prime}>1} \zeta^{2} c_{2_{k^{\prime}+1}}-\left(\delta_{k^{\prime}>1}+\delta_{k^{\prime}>3}\right) \zeta c_{2_{k^{\prime}-1}}-\delta_{k^{\prime}>3} \zeta^{2} c_{2_{k^{\prime}-3}}-\delta_{k^{\prime}>5} \zeta c_{k_{k^{\prime}-5}} \\
& -c_{2_{k^{\prime}+1}}-\delta_{k^{\prime}>1} \zeta c_{2_{k^{\prime}-1}}-\delta_{k^{\prime}>3} c_{2_{k^{\prime}-3}} \\
& =c_{k_{k^{\prime}+5}}+\delta_{k^{\prime}>1} \zeta c_{2_{k^{\prime}+3}}+\left(\delta_{k^{\prime}>1}+\delta_{k^{\prime}>3}\right) c_{2_{k^{\prime}+1}}+\delta_{k^{\prime}>3} c_{2_{k^{\prime}-3}}+\delta_{k^{\prime}>3} \zeta c_{2_{k^{\prime}-1}} \\
& +\delta_{k^{\prime}>5} c_{k_{k^{\prime}-3}}+\delta_{k^{\prime}>5} \zeta c_{2_{k^{\prime}-5}}+\delta_{k^{\prime}>7} c_{2_{k^{\prime}-7}} \\
& =c_{k_{k^{\prime}+5}}+\delta_{k^{\prime}>1} \zeta c_{2_{k^{\prime}+3}}+\left(\delta_{k^{\prime}>1}+\delta_{k^{\prime}>3}\right) c_{2_{k^{\prime}+1}} \\
& +\delta_{k^{\prime}>3} \zeta c_{k_{k^{\prime}-1}}+\left(\delta_{k^{\prime}>3}+\delta_{k^{\prime}>5}\right) c_{2_{k^{\prime}-3}}+\delta_{k^{\prime}>5} \zeta c_{2_{k^{\prime}-5}}+\delta_{k^{\prime}>7} c_{2_{k^{\prime}-7}} .
\end{aligned}
$$

A similar argument applies for $k \geq 4$. This proves (a).

(b) is obtained by multiplying both sides of (a) by $c_{1}$ on the left; (c),(d) are obtained by applying $h \mapsto h^{b}$ to both sides of (a),(b). We prove (e). We have

$$
c_{2_{2 k+2}} c_{1_{k^{\prime}}}=c_{2_{2 k+1}} c_{1} c_{1_{k^{\prime}}}=f_{1} c_{2_{2 k+1}} c_{1_{k^{\prime}}}
$$

and the last expression can be computed from (a). This proves (e). Similarly, (f) follows from (b); (g) is a special case of 6.6. The proposition is proved. 
17.5. From 7.4,7.6 we see that the function $\Delta: W \rightarrow \mathbf{N}$ has the following values:

$$
\begin{aligned}
& \Delta\left(2_{2 k}\right)=k L_{1}+k L_{2}, \\
& \Delta\left(2_{2 k+1}\right)=-k L_{1}+(k+1) L_{2}, \\
& \Delta\left(1_{1}\right)=L_{1}, \\
& \Delta\left(1_{2 k+1}\right)=(k-1) L_{1}+k L_{2}, \text { if } k \geq 1, \\
& \Delta\left(1_{2 k}\right)=k L_{1}+k L_{2} .
\end{aligned}
$$

It follows that $\mathrm{P} 1$ holds and that $\mathcal{D}$ consists of $2_{0}=1_{0}, 2_{1}, 1_{1}, 1_{3}$. Thus, P6 holds.

The formulas in $17.3,17.4$ determine $h_{x, y, z}$ for all $x, y, z$ except when $x=1$ or $y=1$, in which case $h_{1, y, z}=\delta_{y, z}, h_{x, 1, z}=\delta_{x, z}$. From these formulas we see that the triples $(x, y, d)$ with $d \in \mathcal{D}, \gamma_{x, y, d} \neq 0$ are:

$\left(2_{2 k+1}, 2_{2 k+1}, 2_{1}\right),\left(1_{2 k+2}, 2_{2 k+2}, 1_{3}\right),\left(1_{1}, 1_{1}, 1_{1}\right)$,

$(1,1,1),\left(2_{2 k+2}, 1_{2 k+2}, 2_{1}\right),\left(1_{2 k+3}, 1_{2 k+3}, 1_{3}\right)$,

where $k \geq 0$. This implies that P2,P3 hold. From the results in 8.8 we see that P4,P9,P13 hold. From 14.5 we see that P5 holds. From 14.7 we see that P7 holds. From 14.8 we see that P8 holds. From 14.10 we see that P10 holds. From 14.11 we see that P11 holds. From 14.12 we see that P12 holds. From 14.14 we see that P14 holds.

We now verify P15 in our case. With the notation in 14.15 , it is enough to show that, if $a, b \in\{1,2\}, w \in W, s_{a} w>w, w s_{b}>w$, then

$$
\left(c_{a} e_{w}\right) c_{b}^{\prime}-c_{a}\left(e_{w} c_{b}^{\prime}\right) \in \tilde{\mathcal{H}}_{\geq \mathbf{a}(w)+1} .
$$

Here $c_{a}=c_{s_{a}}, c_{b}^{\prime}=c_{s_{b}}^{\prime}$. If $a$ or $b$ is 1 , then from 17.2 we have $\left(c_{a} e_{w}\right) c_{b}^{\prime}-c_{a}\left(e_{w} c_{b}^{\prime}\right)=$ 0 . Hence we may assume that $a=b=2$ and $w=1_{2 k+1}$. Using 17.2 we have

$$
\begin{aligned}
& c_{2}\left(e_{1_{2 k+1}} c_{2}^{\prime}\right)=c_{2}\left(e_{1_{2 k+2}}+\delta_{k>0}\right) \zeta^{\prime} e_{1_{2 k}}+\delta_{k>1} e_{1_{2 k-2}} \\
& =e_{2_{2 k+3}}+\zeta e_{2_{2 k+1}}+\delta_{k>0} e_{2_{2 k-1}}+\delta_{k>0} \zeta^{\prime} e_{2_{2 k+1}}+\delta_{k>0} \zeta \zeta^{\prime} e_{2_{2 k-1}} \\
& +\delta_{k>1} \zeta^{\prime} e_{2_{2 k-3}}+\delta_{k>1} e_{2_{2 k-1}}+\delta_{k>1} \zeta e_{2_{2 k-3}}+\delta_{k>2} e_{2_{2 k-5}} \\
& =e_{2_{2 k+3}}+\zeta e_{2_{2 k+1}}+\delta_{k>0} \zeta^{\prime} e_{2_{2 k+1}}+\delta_{k>0} e_{2_{2 k-1}}+\delta_{k>1} e_{2_{2 k-1}} \\
& +\delta_{k>0} \zeta \zeta^{\prime} e_{2_{2 k-1}}+\delta_{k>1}\left(\zeta+\zeta^{\prime}\right) e_{2_{2 k-3}}+\delta_{k>2} e_{2_{2 k-5}} .
\end{aligned}
$$

Similarly,

$$
\begin{aligned}
& \left.\left(c_{2} e_{1_{2 k+1}}\right) c_{2}^{\prime}=e_{2_{2 k+3}}+\zeta^{\prime} e_{2_{2 k+1}}+\delta_{k>0} \zeta e_{2_{2 k+1}}+\delta_{k>0}\right) e_{2_{2 k-1}}+\delta_{k>1} e_{2_{2 k-1}} \\
& +\delta_{k>0} \zeta \zeta^{\prime} e_{2_{2 k-1}}+\delta_{k>1}\left(\zeta+\zeta^{\prime}\right) e_{2_{2 k-3}}+\delta_{k>2} e_{2_{2 k-5}} .
\end{aligned}
$$

Hence

$$
c_{2}\left(e_{1_{2 k+1}} c_{2}^{\prime}\right)-\left(c_{2} e_{1_{2 k+1}}\right) c_{2}^{\prime}=\left(\zeta-\zeta^{\prime}\right)\left(1-\delta_{k>0}\right) e_{2_{2 k+1}} .
$$

If $k>0$, the right hand side is zero. Thus we may assume that $k=0$. In this case,

$$
c_{2}\left(e_{1_{1}} c_{2}^{\prime}\right)-\left(c_{2} e_{1_{1}}\right) c_{2}^{\prime}=\left(\zeta-\zeta^{\prime}\right) e_{2_{1}} .
$$

We have $\mathbf{a}\left(1_{1}\right)=L_{1}<L_{2}=\mathbf{a}\left(2_{1}\right)$. This completes the verification of P15 in our case. 


\section{THE RING $J$}

18.1. In this section we assume that $W$ is tame and that P1-P15 in $\S 14$ are valid.

Theorem 18.2. (a) $W$ has only finitely many left cells.

(b) $W$ has only finitely many right cells.

(c) W has only finitely many two-sided cells.

(d) $\mathcal{D}$ is a finite set.

We prove (a). Since $\mathbf{a}(w)$ is bounded above it is enough to show that, for any $a \in \mathbf{N}, \mathbf{a}^{-1}(a)$ is a union of finitely many left cells. By $\mathrm{P} 4, \mathbf{a}^{-1}(a)$ is a union of left cells. Let $\mathcal{H}^{1}$ be the $\mathbf{Z}$-algebra $\mathbf{Z} \otimes_{\mathcal{A}} \mathcal{H}$ where $\mathbf{Z}$ is regarded as an $\mathcal{A}$-algebra via $v \mapsto 1$. We write $c_{w}$ instead of $1 \otimes c_{w}$. For any $a^{\prime} \geq 0$ let $\mathcal{H}_{\geq a^{\prime}}^{1}$ be the subgroup of $\mathcal{H}^{1}$ spanned by $\left\{c_{w} ; \mathbf{a}(w) \geq a^{\prime}\right\}$ (a two-sided ideal of $\mathcal{H}^{1}$, by $\mathrm{P} 4$ ). We have a direct sum decomposition

$$
\mathcal{H}_{\geq a}^{1} / \mathcal{H}_{\geq a+1}^{1}=\oplus_{\Gamma} E_{\Gamma}
$$

where $\Gamma$ runs over the left cells contained in $\mathbf{a}^{-1}(a)$ and $E_{\Gamma}$ is generated as a group by the images of $c_{w}, w \in \Gamma$; these images form a $\mathbf{Z}$-basis of $E_{\Gamma}$. Now $\mathcal{H}_{\geq a}^{1} / \mathcal{H}_{\geq a+1}^{1}$ inherits a left $\mathcal{H}^{1}$-module structure from $\mathcal{H}^{1}$ and (by P9) each $E_{\Gamma}$ is a $\mathcal{H}^{1}$-submodule. Since $W$ is tame, there exists a finitely generated abelian subgroup $W_{1}$ of finite index of $W$. Now $\mathcal{H}^{1}=\mathbf{Z}[W]$ contains $\mathbf{Z}\left[W_{1}\right]$ as a subring. Since $\mathcal{H}_{\geq a}^{1} / \mathcal{H}_{\geq a+1}^{1}$ is a subquotient of $\mathcal{H}^{1}$ (a finitely generated $\mathbf{Z}\left[W_{1}\right]$-module) and $\mathbf{Z}\left[W_{1}\right]$ is a noetherian ring, it follows that $\mathcal{H}_{\geq a}^{1} / \mathcal{H}_{\geq a+1}^{1}$ is a finitely generated $\mathbf{Z}\left[W_{1}\right]$-module. Hence in the direct sum decomposition (e) with only non-zero summands, the number of summands must be finite. This proves (a).

Since any right cell is of the form $\Gamma^{-1}$ where $\Gamma$ is a left cell, we see that (b) follows from (a). Since any two-sided cell is a union of left cells, we see that (c) follows from (a). From P16 we see that (d) follows from (a). The theorem is proved.

18.3. Let $J$ be the free abelian group with basis $\left(t_{w}\right)_{w \in W}$. We set

$$
t_{x} t_{y}=\sum_{z \in W} \gamma_{x, y, z^{-1}} t_{z}
$$

The sum is finite since $\gamma_{x, y, z^{-1}} \neq 0 \Longrightarrow h_{x, y, z} \neq 0$ and this implies that $z$ runs through a finite set (for fixed $x, y$ ). We show that this defines an (associative) ring structure on $J$. We must check the identity

$$
\sum_{z} \gamma_{x, y, z^{-1}} \gamma_{z, u, u^{\prime-1}}=\sum_{w} \gamma_{y, u, w^{-1}} \gamma_{x, w, u^{\prime-1}}
$$

for any $x, y, u, u^{\prime} \in W$. From P8,P4 we see that both sides of (a) are 0 unless

$$
\mathbf{a}(x)=\mathbf{a}(y)=\mathbf{a}(u)=\mathbf{a}\left(u^{\prime}\right)=a
$$


for some $a \in \mathbf{N}$. Hence we may assume that (b) holds. By P8,P4, in the first sum in (a) we may assume that $\mathbf{a}(z)=a$ and in the second sum in (a) we may assume that $\mathbf{a}(w)=a$. The equation $\left(c_{x} c_{y}\right) c_{u}=c_{x}\left(c_{y} c_{u}\right)$ in $\mathcal{H}$ implies

$$
\sum_{z} h_{x, y, z} h_{z, u, u^{\prime}}=\sum_{w} h_{y, u, w} h_{x, w, u^{\prime}}
$$

If $h_{x, y, z} h_{z, u, u^{\prime}} \neq 0$ then $u^{\prime} \leq_{\mathcal{R}} z \leq_{\mathcal{R}} x$ hence, by $\mathrm{P} 4, \mathbf{a}\left(u^{\prime}\right) \geq \mathbf{a}(z) \geq \mathbf{a}(x)$ and $\mathbf{a}(z)=a$. Hence in the first sum in (c) we may assume that $\mathbf{a}(z)=a$. Similarly in the second sum in (c) we may assume that $\mathbf{a}(w)=a$. Taking the coefficient of $v^{2 \mathbf{a}(z)}$ in both sides of (c) we find (a).

The ring $J$ has a unit element $\sum_{d \in \mathcal{D}} n_{d} t_{d}$. Here $n_{d}= \pm 1$ is as in $14.1(\mathrm{a})$, see P5. (The sum is well defined by 18.2(d).) Let us check that $t_{x} \sum_{d} n_{d} t_{d}=t_{x}$ for $x \in W$. This is equivalent to the identity $\sum_{d} n_{d} \gamma_{x, d, z^{-1}}=\delta_{z, x}$. By P7 this is equivalent to $\sum_{d} n_{d} \gamma_{z^{-1}, x, d}=\delta_{z, x}$. This follows from P2,P3,P5. The equality $\left(\sum_{d} n_{d} t_{d}\right) t_{x}=t_{x}$ is checked in a similar way.

For any subset $X$ of $W$, let $J^{X}$ be the subgroup of $J$ generated by $\left\{t_{x} ; x \in X\right\}$. If $\mathbf{c}$ is a two-sided cell of $W, L$ then, by $\mathrm{P} 8, J^{\mathbf{c}}$ is a subring of $J$ and $J=\oplus_{\mathbf{c}} J^{\mathbf{c}}$ is a direct sum decomposition of $J$ as a ring. The unit element of $J^{\mathbf{c}}$ is $\sum_{d \in \mathcal{D} \cap \mathbf{c}} n_{d} t_{d}$. Similarly, if $\Gamma$ is a left cell of $W, L$ then $J^{\Gamma \cap \Gamma^{-1}}$ is a subring of $J$ with unit element $n_{d} t_{d}$ where $d \in \mathcal{D} \cap \Gamma$.

Proposition 18.4. Assume that we are in the setup of 15.1. Let $x, y \in W$.

(a) The condition $x \sim_{\mathcal{L}} y$ is equivalent to the condition that $t_{x} t_{y^{-1}} \neq 0$ and to the condition that, for some $u, t_{y}$ appears with $\neq 0$ coefficient in $t_{u} t_{x}$.

(b) The condition $x \sim_{\mathcal{R}} y$ is equivalent to the condition that $t_{x^{-1}} t_{y} \neq 0$ and to the condition that, for some $u, t_{y}$ appears with $\neq 0$ coefficient in $t_{x} t_{u}$.

(c) The condition $x \sim_{\mathcal{L} \mathcal{R}} y$ is equivalent to the condition that $t_{x} t_{u} t_{y} \neq 0$ for some $u$ and to the condition that, for some $u, u^{\prime}, t_{y}$ appears with $\neq 0$ coefficient in $t_{u^{\prime}} t_{x} t_{u}$.

Let $J^{+}=\sum_{z} \mathbf{N} t_{z}$. By 15.1(a) we have $J^{+} J^{+} \subset J^{+}$.

We prove (a). The second condition is equivalent to $\gamma_{x, y^{-1}, u} \neq 0$ for some $u$; the third condition is equivalent to $\gamma_{x, u, y^{-1}} \neq 0$ for some $u$. These conditions are equivalent by P7.

Assume that $\gamma_{x, y^{-1}, u} \neq 0$ for some $u$. Using P8 we deduce that $x \sim_{\mathcal{L}} y$.

Assume now that $x \sim_{\mathcal{L}} y$. Let $d \in \mathcal{D}$ be such that $x \sim_{\mathcal{L}} d$. Then we have also $y \sim_{\mathcal{L}} d$. By P13 we have $\gamma_{x^{-1}, x, d} \neq 0, \gamma_{y^{-1}, y, d} \neq 0$. Hence $\gamma_{x^{-1}, x, d}=1, \gamma_{y^{-1}, y, d}=$ 1. Hence $t_{x^{-1}} t_{x} \in t_{d}+J^{+}, t_{y^{-1}} t_{y} \in t_{d}+J^{+}$. Since $t_{d} t_{d}=t_{d}$, it follows that $t_{x^{-1}} t_{x} t_{y^{-1}} t_{y} \in t_{d} t_{d}+J^{+}=t_{d}+J^{+}$. In particular, $t_{x} t_{y^{-1}} \neq 0$. This proves (a).

The proof of (b) is entirely similar.

We prove (c). Using the associativity of $J$ we see that the third condition on $x, y$ is a transitive relation on $W$. Hence to prove that the first condition implies the third condition we may assume that either $x \sim_{\mathcal{L}} y$ or $x \sim_{\mathcal{R}} y$, in which case this 
follows from (a) or (b). The fact that the third condition implies the first condition also follows from (a),(b). Thus the first and third condition are equivalent.

Assume that $t_{x} t_{u} t_{y} \neq 0$ for some $u$. By (a), (b) we then have $x \sim_{\mathcal{L}} u^{-1}, u^{-1} \sim_{\mathcal{R}}$ $y$. Hence $x \sim_{\mathcal{L R}} y$.

Conversely, assume that $x \sim_{\mathcal{L R}} y$. By P14 we have $x \sim_{\mathcal{L R}} y^{-1}$. By the earlier part of the proof, $t_{y^{-1}}$ appears with $\neq 0$ coefficient in $t_{u^{\prime}} t_{x} t_{u}$ for some $u, u^{\prime}$. We have $t_{u^{\prime}} t_{x} t_{u} \in a t_{y^{-1}}+J^{+}$where $a>0$. Hence $t_{u^{\prime}} t_{x} t_{u} t_{y} \in a t_{y^{-1}} t_{y}+J^{+}$. Since $t_{y^{-1}} t_{y}$ has a coefficient 1 and the other coefficients are $\geq 0$, it folows that $t_{u^{\prime}} t_{x} t_{u} t_{y} \neq 0$. Thus, $t_{x} t_{u} t_{y} \neq 0$. We see that the first and second conditions are equivalent. The proposition is proved.

18.5. Assume now that we are in the setup of 7.1 with $m=\infty$ and $L_{2}>L_{1}$. From the formulas in $17.3,17.4$ we can determine the multiplication table of $J$. We find

$$
\begin{aligned}
& t_{2_{2 k+1}} t_{2_{2 k^{\prime}+1}}=\sum_{u \in[0, \tilde{k}]} t_{2_{2 k+2 k^{\prime}+1-4 u}}, \\
& t_{1_{2 k+3}} t_{1_{2 k^{\prime}+3}}=\sum_{u \in[0, \tilde{k}]} t_{1_{2 k+2 k^{\prime}+3-4 u}}, \\
& t_{2_{2 k+1}} t_{2_{2 k^{\prime}+2}}=\sum_{u \in[0, \tilde{k}]} t_{2_{2 k+2 k^{\prime}+2-4 u}}, \\
& t_{1_{2 k+3} t_{1_{2 k^{\prime}+2}}}=\sum_{u \in[0, \tilde{k}]} t_{1_{2 k+2 k^{\prime}+2-4 u}}, \\
& t_{2_{2 k+2}} t_{1_{2 k^{\prime}+3}}=\sum_{u \in[0, \tilde{k}]} t_{2_{2 k+2 k^{\prime}+2-4 u}}, \\
& t_{2_{2 k+2}} t_{1_{2 k^{\prime}+2}}=\sum_{u \in[0, \tilde{k}]} t_{2_{2 k+2 k^{\prime}+1-4 u}}, \\
& t_{1_{2 k+2}} t_{2_{2 k^{\prime}+1}}=\sum_{u \in[0, \tilde{k}]} t_{1_{2 k+2 k^{\prime}+2-4 u}}, \\
& t_{1_{2 k+2}} t_{2_{2 k^{\prime}+2}}=\sum_{u \in[0, \tilde{k}]} t_{1_{2 k+2 k^{\prime}+3-4 u}}, \\
& t_{1_{1} t_{1_{1}}=t_{1_{1}},} \\
& t_{1} t_{1}=t_{1} ;
\end{aligned}
$$

here $k, k^{\prime} \geq 0$ and $\tilde{k}=\min \left(k, k^{\prime}\right)$. All other products are 0 .

Let $R$ be the free abelian group with basis $\left(b_{k}\right)_{k \in \mathbf{N}}$. We regard $R$ as a commutative ring with multiplication

$$
b_{k} b_{k^{\prime}}=\sum_{u \in\left[0, \min \left(k, k^{\prime}\right)\right]} b_{k+k^{\prime}-2 u} .
$$

Let $J_{0}=\sum_{w \in W-\left\{1,1_{1}\right\}} \mathbf{Z} t_{w}$. The formulas above show that $J=J_{0} \oplus \mathbf{Z} t_{1} \oplus \mathbf{Z} t_{1_{1}}$ (direct sum of rings) and that the ring $J_{0}$ is isomorphic to the ring of $2 \times 2$ matrices with entries in $R$, via the isomorphism defined by:

$$
t_{2_{2 k+1}} \mapsto\left(\begin{array}{cc}
b_{k} & 0 \\
0 & 0
\end{array}\right), \quad t_{1_{2 k+3}} \mapsto\left(\begin{array}{cc}
0 & 0 \\
0 & b_{k}
\end{array}\right), \quad t_{2_{2 k+2}} \mapsto\left(\begin{array}{cc}
0 & b_{k} \\
0 & 0
\end{array}\right), \quad t_{1_{2 k+2}} \mapsto\left(\begin{array}{cc}
0 & 0 \\
b_{k} & 0
\end{array}\right) .
$$

Note that $R$ is canonically isomorphic to the representation ring of $S L_{2}(\mathbf{C})$ with its canonical basis consisting of irreducible representations.

18.6. Assume that we are in the setup of 7.1 with $m=\infty$ and $L_{2}=L_{1}$. By methods similar (but simpler) to those of $\S 17$ and 18.5, we find

$$
t_{22 k+1} t_{2 k^{\prime}+1}=\sum_{u \in\left[0,2 \min \left(k, k^{\prime}\right)\right]} t_{2_{2 k+2 k^{\prime}+1-2 u}} .
$$


Let $J^{1}$ be the subring of $J$ generated by $t_{2_{2 k+1}}, k \in \mathbf{N}$. While, in 18.5 , the analogue of $J^{1}$ was isomorphic to $R$ as a ring with basis, in the present case, $J^{1}$ is canonically isomorphic to $R^{\prime}$, the subgroup of $R$ generated by $b_{k}$ with $k$ even. (Note that $R^{\prime}$ is a subring of $R$, naturally isomorphic to the representation ring of $P G L_{2}(\mathbf{C})$.)

18.7. In the setup of 7.1 with $m=4$ and $L_{2}=2, L_{1}=1$ (a special case of the situation in $\S 16)$, we have

$$
J=\mathbf{Z} t_{1} \oplus \mathbf{Z} t_{1_{1}} \oplus J_{0} \oplus \mathbf{Z} t_{2_{3}} \oplus \mathbf{Z} t_{2_{4}}
$$

(direct sum of rings) where $J_{0}$ is the subgroup of $J$ generated by $t_{2_{1}}, t_{2_{2}}, t_{1_{2}}, t_{1_{3}}$. The ring $J_{0}$ is isomorphic to the ring of $2 \times 2$ matrices with entries in $\mathbf{Z}$, via the isomorphism defined by:

$$
t_{2_{1}} \mapsto\left(\begin{array}{cc}
1 & 0 \\
0 & 0
\end{array}\right), \quad t_{1_{3}} \mapsto\left(\begin{array}{cc}
0 & 0 \\
0 & 1
\end{array}\right), \quad t_{2_{2}} \mapsto\left(\begin{array}{cc}
0 & 1 \\
0 & 0
\end{array}\right), \quad t_{1_{2}} \mapsto\left(\begin{array}{cc}
0 & 0 \\
1 & 0
\end{array}\right)
$$

Moreover, $t_{1}, t_{1_{1}}, t_{2_{4}}$ are idempotent. On the other hand,

$$
t_{2_{3}} t_{2_{3}}=-t_{2_{3}}
$$

Notice the minus sign! (It is a special case of the computation in 7.8.)

18.8. For any $z \in W$ we set $\hat{n}_{z}=n_{d}$ where $d$ is the unique element of $\mathcal{D}$ such that $d \sim_{\mathcal{L}} z^{-1}$ and $n_{d}= \pm 1$ is as in 14.1(a), see P5. Note that $z \mapsto \hat{n}_{z}$ is constant on right cells.

Theorem 18.9. The $\mathcal{A}$-linear map $\phi: \mathcal{H} \rightarrow J_{\mathcal{A}}=\mathcal{A} \otimes J$ given by

$$
\phi\left(c_{x}^{\dagger}\right)=\sum_{z \in W, d \in \mathcal{D} ; \mathbf{a}(d)=\mathbf{a}(z)} h_{x, d, z} \hat{n}_{z} t_{z} \quad(x \in W)
$$

is a homomorphism of $\mathcal{A}$-algebras with 1 .

Consider the equality

(a) $\sum_{w} h_{x_{1}, x_{2}, w} h_{w, x_{3}, y}^{\prime}=\sum_{w} h_{x_{1}, w, y} h_{x_{2}, x_{3}, w}^{\prime}$

(see P15) with $\mathbf{a}\left(x_{2}\right)=\mathbf{a}(y)=a$. In the left hand side we may assume that $y \leq_{\mathcal{R}} w \leq_{\mathcal{L}} x_{2}$ hence (by P4) $\mathbf{a}(y) \geq \mathbf{a}(w) \geq \mathbf{a}\left(x_{2}\right)$, hence $\mathbf{a}(w)=a$. Similarly in the right hand side we may assume that $\mathbf{a}(w)=a$. Picking the coefficient of $v^{\prime a}$ in both sides of (a) gives

(b) $\sum_{w} h_{x_{1}, x_{2}, w} \gamma_{w, x_{3}, y^{-1}}=\sum_{w} h_{x_{1}, w, y} \gamma_{x_{2}, x_{3}, w^{-1}}$.

Let $x, x^{\prime} \in W$. The desired identity $\phi\left(c_{x}^{\dagger} c_{x^{\prime}}^{\dagger}\right)=\phi\left(c_{x}^{\dagger}\right) \phi\left(c_{x^{\prime}}^{\dagger}\right)$ is equivalent to

$$
\sum_{\substack{w \in W, d \in \mathcal{D} \\ \mathbf{a}(d)=a^{\prime}}} h_{x, x^{\prime}, w} h_{w, d, u} \hat{n}_{u}=\sum_{\substack{z, z^{\prime} \in W, d, d^{\prime} \in \mathcal{D} \\ \mathbf{a}(d)=\mathbf{a}(z) \\ \mathbf{a}\left(d^{\prime}\right)=\mathbf{a}\left(z^{\prime}\right)}} h_{x, d, z} h_{x^{\prime}, d^{\prime}, z^{\prime}} \gamma_{z, z^{\prime}, u^{-1}} \hat{n}_{z} \hat{n}_{z^{\prime}}
$$


for any $u \in W$ such that $\mathbf{a}(u)=a^{\prime}$. In the right hand we may assume that

$$
\mathbf{a}(d)=\mathbf{a}(z)=\mathbf{a}\left(d^{\prime}\right)=\mathbf{a}\left(z^{\prime}\right)=a^{\prime} \text { and } \hat{n}_{z}=\hat{n}_{u}
$$

(by $\mathrm{P} 8, \mathrm{P} 4)$. Hence the right hand side can be rewritten (using (b)):

$$
\begin{aligned}
& \sum_{\begin{array}{c}
z^{\prime} \in W, d, d^{\prime} \in \mathcal{D} \\
\mathbf{a}(d)=\mathbf{a}\left(d^{\prime}\right)=\mathbf{a}\left(z^{\prime}\right)=a^{\prime}
\end{array}} h_{x^{\prime}, d^{\prime}, z^{\prime}} \sum_{z ; \mathbf{a}(z)=a^{\prime}} h_{x, d, z} \gamma_{z, z^{\prime}, u} \hat{n}_{u} \hat{n}_{z^{\prime}} \\
& =\sum_{\substack{z^{\prime} \in W, d, d^{\prime} \in \mathcal{D} \\
\mathbf{a}(d)=\mathbf{a}\left(d^{\prime}\right)=\mathbf{a}\left(z^{\prime}\right)=a^{\prime}}} h_{x^{\prime}, d^{\prime}, z^{\prime}} \sum_{w ; \mathbf{a}(w)=a^{\prime}} h_{x, w, u} \gamma_{d, z^{\prime}, w^{-1}} \hat{n}_{u} \hat{n}_{z^{\prime}} .
\end{aligned}
$$

By P2,P3,P5, this equals

$$
\sum_{z^{\prime} \in W, d^{\prime} \in \mathcal{D} ; \mathbf{a}\left(d^{\prime}\right)=\mathbf{a}\left(z^{\prime}\right)=a^{\prime}} h_{x^{\prime}, d^{\prime}, z^{\prime}} h_{x, z^{\prime}, u} \hat{n}_{u}
$$

which by the identity $\left(c_{x} c_{x^{\prime}}\right) c_{d^{\prime}}=c_{x}\left(c_{x^{\prime}} c_{d^{\prime}}\right)$ equals

$$
\sum_{w \in W, d^{\prime} \in \mathcal{D} ; \mathbf{a}\left(d^{\prime}\right)=a^{\prime}} h_{x, x^{\prime}, w} h_{w, d^{\prime}, u} \hat{n}_{u} .
$$

Thus $\phi$ is compatible with multiplication.

Next we show that $\phi$ is compatible with the unit elements of the two algebras. An equivalent statement is that for any $z \in W$ such that $\mathbf{a}(z)=a$, the sum $\sum_{d \in \mathcal{D} ; \mathbf{a}(d)=a} h_{1, d, z} \hat{n}_{z}$ equals $n_{z}$ if $z \in \mathcal{D}$ and is 0 if $z \notin \mathcal{D}$. This is clear since $h_{1, d, z}=\delta_{z, d}$.

18.10. If we identify the $\mathcal{A}$-modules $\mathcal{H}$ and $J_{\mathcal{A}}$ via $c_{w}^{\dagger} \mapsto \hat{n}_{w} t_{w}$, the obvious left $J_{\mathcal{A}}$-module structure on $J_{\mathcal{A}}$ becomes the left $J_{\mathcal{A}}$-module structure on $\mathcal{H}$ given by

$$
t_{x} * c_{w}^{\dagger}=\sum_{z \in W} \gamma_{x, w, z^{-1}} \hat{n}_{w} \hat{n}_{z} c_{z}^{\dagger}
$$

Let $\mathcal{H}_{a}=\oplus_{w ; \mathbf{a}(w)=a} \mathcal{A} c_{w}^{\dagger}, \mathcal{H}_{\geq a}=\oplus_{w ; \mathbf{a}(w) \geq a} \mathcal{A} c_{w}^{\dagger}$. We have $t_{x} * c_{w}^{\dagger} \in \mathcal{H}_{\mathbf{a}(w)}$ for all $x, w$. For any $h \in \mathcal{H}, w \in W$ we have

$$
h c_{w}^{\dagger}=\phi(h) * c_{w}^{\dagger} \quad \bmod \mathcal{H}_{\geq \mathbf{a}(w)+1} .
$$

Indeed, we may assume that $h=c_{x}^{\dagger}$. Using 18.9(b), we have

$$
\begin{aligned}
& \phi\left(c_{x}^{\dagger}\right) * c_{w}^{\dagger}=\sum_{\substack{d \in \mathcal{D}, z \\
\mathbf{a}(d)=\mathbf{a}(z)}} h_{x, d, z} \hat{n}_{z} t_{z} * c_{w}^{\dagger} \\
& =\sum_{\substack{d \in \mathcal{D}, z, u \\
\mathbf{a}(d)=\mathbf{a}(z)}} h_{x, d, z} \gamma_{z, w, u^{-1}} \hat{n}_{z} \hat{n}_{w} \hat{n}_{u} c_{u}^{\dagger}=\sum_{\substack{d \in \mathcal{D}, z, u \\
\mathbf{a}(d)=\mathbf{a}(w)=\mathbf{a}(u)}} h_{x, d, z} \gamma_{z, w, u}-1 \hat{n}_{w} c_{u}^{\dagger} \\
& =\sum_{\substack{d \in \mathcal{D}, t, u \\
\mathbf{a}(d)=\mathbf{a}(w)=\mathbf{a}(u)}} h_{x, t, u} \gamma_{d, w, t^{-1}} \hat{n}_{w} c_{u}^{\dagger}=\sum_{\substack{d \in \mathcal{D}, u \\
\mathbf{a}(d)=\mathbf{a}(w)=\mathbf{a}(u)}} h_{x, w, u} \gamma_{d, w, w-1} \hat{n}_{w} c_{u}^{\dagger} \\
& =\sum_{\substack{u \\
\mathbf{a}(w)=\mathbf{a}(u)}} h_{x, w, u} c_{u}^{\dagger}=c_{x}^{\dagger} c_{w}^{\dagger} \bmod \mathcal{H}_{\geq \mathbf{a}(w)+1},
\end{aligned}
$$


as required.

18.11. Let $\mathcal{A} \rightarrow R$ be a ring homomorphism of $\mathcal{A}$ into a commutative ring $R$ with 1. Let $\mathcal{H}_{R}=R \otimes_{\mathcal{A}} \mathcal{H}, J_{R}=R \otimes_{\mathcal{A}}\left(J_{\mathcal{A}}\right)=R \otimes J, \mathcal{H}_{R, \geq a}=R \otimes_{\mathcal{A}} \mathcal{H}_{\geq a}$. Then $\phi$ extends to a homomorphism of $R$-algebras $\phi_{R}: \mathcal{H}_{R} \rightarrow J_{R}$. The $J_{\mathcal{A}}$-module in 18.10 extends to a $J_{A}$-module structure on $\mathcal{H}_{R}$ denoted again by $*$. From 18.10 (a) we deduce

(a) $h c_{w}^{\dagger}=\phi_{R}(h) * c_{w}^{\dagger} \bmod \mathcal{H}_{R, \geq \mathbf{a}(w)+1}$ for any $h \in \mathcal{H}_{R}, w \in W$.

Proposition 18.12. (a) If $N$ is a bound for $W, L$, then $\left(\operatorname{ker} \phi_{R}\right)^{N+1}=0$.

(b) If $R=R_{0}\left[v, v^{-1}\right]$ where $R_{0}$ is a commutative ring with $1, v$ is an indeterminate and $\mathcal{A} \rightarrow R$ is the obvious ring homomorphism, then $\operatorname{ker} \phi_{R}=0$.

We prove (a). If $h \in \operatorname{ker} \phi_{R}$ then by 18.11(a), we have $h \mathcal{H}_{R, \geq a} \subset \mathcal{H}_{R, \geq a+1}$ for any $a \geq 0$. Applying this repeatedly, we see that, if $h_{1}, h_{2}, \ldots, h_{N+1} \in \mathcal{H}$, we have $h_{1} h_{2} \ldots h_{N+1} \in \mathcal{H}_{R, \geq N+1}=0$. This proves (a).

We prove (b). Let $h=\sum_{x} p_{x} c_{x}^{\dagger} \in \operatorname{ker} \phi_{R}$ where $p_{x} \in R$. Assume that $h \neq 0$. Then $p_{x} \neq 0$ for some $x$. We can find $a \geq 0$ such that $p_{x} \neq 0 \Longrightarrow \mathbf{a}(x) \geq a$ and $X=\left\{x \in W ; p_{x} \neq 0, \mathbf{a}(x)=a\right\}$ is non-empty. We can find $b \in \mathbf{Z}$ such that $p_{x} \in v^{b} \mathbf{Z}\left[v^{-1}\right]$ for all $x \in X$ and such that $X^{\prime}=\left\{x \in X ; \pi_{b}\left(p_{x}\right) \neq 0\right\}$ is non-empty. Let $x_{0} \in X^{\prime}$. We can find $d \in \mathcal{D}$ such that $\gamma_{x_{0}, d, x_{0}^{-1}}=\gamma_{x_{0}^{-1}, x_{0}, d} \neq 0$. We have $h c_{d}^{\dagger}=\sum_{x} p_{x} c_{x}^{\dagger} c_{d}^{\dagger}$. If $\mathbf{a}(x)>a$, then $c_{x}^{\dagger} c_{d}^{\dagger} \in \mathcal{H}_{R, \geq a+1}$. Hence $h c_{d}^{\dagger}=\sum_{x \in X} p_{x} c_{x}^{\dagger} c_{d}^{\dagger}$ $\bmod \mathcal{H}_{R, \geq a+1}$. Since $\phi_{R}(h)=0$, from $18.11(\mathrm{a})$ we have $h c_{d}^{\dagger} \in \bmod \mathcal{H}_{R, \geq a+1}$. It follows that $\sum_{x \in X} p_{x} c_{x}^{\dagger} c_{d}^{\dagger} \in \mathcal{H}_{R, \geq a+1}$. In particular the coefficient of $c_{x_{0}}^{\dagger}$ in $\sum_{x \in X} p_{x} c_{x}^{\dagger} c_{d}^{\dagger}$ is 0 . In other words, $\sum_{x \in X} p_{x} h_{x, d, x_{0}}=0$. The coefficient of $v^{a+b}$ in the last sum is

$$
\sum_{x \in X} \pi_{b}\left(p_{x}\right) \gamma_{x, d, x_{0}^{-1}}=\pi_{b}\left(p_{x_{0}}\right) \gamma_{x_{0}, d, x_{0}^{-1}}
$$

and this is on the one hand 0 and on the other hand is non-zero since $\pi_{b}\left(p_{x_{0}}\right) \neq 0$ and $\gamma_{x_{0}, d, x_{0}^{-1}} \neq 0$, by the choice of $x_{0}, d$. This contradiction completes the proof.

\section{Algebras With trace FORM}

19.1. Let $R$ be a field and let $A$ be an associative $R$-algebra with 1 of finite dimension over $R$. We assume that $A$ is semisimple and split over $R$ and that we are given a trace form on $A$ that is, an $R$-linear map $\tau: A \rightarrow R$ such that $\left(a, a^{\prime}\right)=$ $\tau\left(a a^{\prime}\right)=\tau\left(a^{\prime} a\right)$ is a non-degenerate (symmetric) $R$-bilinear form $():, A \times A \rightarrow R$. Note that $\left(a a^{\prime}, a^{\prime \prime}\right)=\left(a, a^{\prime} a^{\prime \prime}\right)$ for all $a, a^{\prime}, a^{\prime \prime}$ in $A$. Let $\operatorname{Mod} A$ be the category whose objects are left $A$-modules of finite dimension over $R$. We write $E \in \operatorname{Irr} A$ for " $E$ is a simple object of $\operatorname{Mod} A$ ".

Let $\left(a_{i}\right)_{i \in I}$ be an $R$-basis of $A$. Define an $R$-basis $\left(a_{i}^{\prime}\right)_{i \in I}$ of $A$ by $\left(a_{i}, a_{j}^{\prime}\right)=\delta_{i j}$. Then

(a) $\sum_{i} a_{i} \otimes a_{i}^{\prime} \in A \otimes A$ is independent of the choice of $\left(a_{i}\right)$. 
Proposition 19.2. (a) We have $\sum_{i} \tau\left(a_{i}\right) a_{i}^{\prime}=1$.

(b) If $E \in \operatorname{Irr} A$, then $\sum_{i} \operatorname{tr}\left(a_{i}, E\right) a_{i}^{\prime}$ is in the centre of $A$. It acts on $E$ as a scalar $f_{E} \in R$ times the identity and on $E^{\prime} \in \operatorname{Irr} A$, not isomorphic to $E$, as zero. Moreover, $f_{E}$ does not depend on the choice of $\left(a_{i}\right)$.

(c) One can attach uniquely to each $E \in \operatorname{Irr} A$ a scalar $g_{E} \in R$ (depending only on the isomorphism class of $E)$, so that

$\sum_{E} g_{E} \operatorname{tr}(a, E)=\tau(a)$ for all $a \in A$, where the sum is taken over all $E \in \operatorname{Irr} A$ up to isomorphism.

(d) For any $E \in \operatorname{Irr} A$ we have $f_{E} g_{E}=1$. In particular, $f_{E} \neq 0, g_{E} \neq 0$.

(e) If $E, E^{\prime} \in \operatorname{Irr} A$, then $\sum_{i} \operatorname{tr}\left(a_{i}, E\right) \operatorname{tr}\left(a_{i}^{\prime}, E^{\prime}\right)$ is $f_{E} \operatorname{dim} E$ if $E, E^{\prime}$ are isomorphic and is 0 , otherwise.

Let $A=\oplus_{n=1}^{t} A_{n}$ be the decomposition of $A$ as a sum of simple algebras. Let $\tau_{n}: A_{n} \rightarrow R$ be the restriction of $\tau$. Then $\tau_{n}$ is a trace form for $A_{n}$, whose associated form is the restriction of $($,$) and \left(A_{n}, A_{n^{\prime}}\right)=0$ for $n \neq n^{\prime}$. Hence we can choose $\left(a_{i}\right)$ so that each $a_{i}$ is contained in some $A_{n}$ and then $a_{i}^{\prime}$ will be contained in the same $A_{n}$ as $a_{i}^{\prime}$.

We prove (a). From 19.1(a) we see that $\sum_{i} \tau\left(a_{i}\right) a_{i}^{\prime}$ is independent of the choice of $\left(a_{i}\right)$. Hence we may choose $\left(a_{i}\right)$ as in the first paragraph of the proof. We are thus reduced to the case where $A$ is simple. In that case the assertion is easily verified.

We prove (b). From 19.1(a) we see that $\sum_{i} \operatorname{tr}\left(a_{i}, E\right) a_{i}^{\prime}$ is independent of the choice of $\left(a_{i}\right)$. Hence we may choose $\left(a_{i}\right)$ as in the first paragraph of the proof. We are thus reduced to the case where $A$ is simple. In that case the assertion is easily verified.

We prove (c). It is enough to note that $a \mapsto \operatorname{tr}(a, E)$ form a basis of the space of $R$-linear functions $A \rightarrow R$ which vanish on all $a a^{\prime}-a^{\prime} a$ and $\tau$ is such a function.

We prove (d). We consider the equation in (c) for $a=a_{i}$, we multiply both sides by $a_{i}^{\prime}$ and sum over $i$. Using (a), we obtain

$$
\sum_{i} \sum_{E} g_{E} \operatorname{tr}\left(a_{i}, E\right) a_{i}^{\prime}=\sum_{i} \tau\left(a_{i}\right) a_{i}^{\prime}=1
$$

Hence $\sum_{E} g_{E} \sum_{i} \operatorname{tr}\left(a_{i}, E\right) a_{i}^{\prime}=1$. By (b), the left hand side acts on a $E^{\prime} \in \operatorname{Irr} A$ as a scalar $g_{E^{\prime}} f_{E^{\prime}}$ times the identity. This proves (d).

(e) follows immediately from (b). The proposition is proved.

19.3. Now let $A^{\prime}$ be a semisimple subalgebra of $A$ such that $\tau^{\prime}$, the restriction of $\tau$ to $A^{\prime}$ is a trace form of $A^{\prime}$. (We do not assume that the unit element $1_{A^{\prime}}$ of $A^{\prime}$ coincides to the unit element 1 of $A$.) If $E \in \operatorname{Mod} A$ then $1_{A^{\prime}} E$ is naturally an object of $\operatorname{Mod} A^{\prime}$. Hence if $E^{\prime} \in \operatorname{Irr} A^{\prime}$, then the multiplicity $\left[E^{\prime}: 1_{A^{\prime}} E\right]$ of $E^{\prime}$ in $1_{A^{\prime}} E^{\prime}$ is well defined.

Note that, if $a^{\prime} \in A^{\prime}$, then $\operatorname{tr}\left(a^{\prime}, 1_{A^{\prime}} E\right)=\operatorname{tr}\left(a^{\prime}, E\right)$. 
Lemma 19.4. Let $E^{\prime} \in \operatorname{Irr} A^{\prime}$. We have $g_{E^{\prime}}=\sum_{E}\left[E^{\prime}: 1_{A^{\prime}} E\right] g_{E}$, sum over all $E \in \operatorname{Irr} A$ (up to isomorphism).

By the definition of $g_{E^{\prime}}$, it is enough to show that

(a) $\sum_{E^{\prime}} \sum_{E}\left[E^{\prime}: 1^{\prime} E\right] g_{E} \operatorname{tr}\left(a^{\prime}, E^{\prime}\right)=\tau\left(a^{\prime}\right)$

for any $a^{\prime} \in A^{\prime}$. Here $E^{\prime}$ (resp. $E$ ) runs over the isomorphism classes of simple objects of $\operatorname{Mod} A^{\prime}(\operatorname{resp} . \operatorname{Mod} A)$. The left hand of (a) is

$$
\sum_{E} g_{E} \sum_{E^{\prime}}\left[E^{\prime}: 1^{\prime} E\right] \operatorname{tr}\left(a^{\prime}, E^{\prime}\right)=\sum_{E} g_{E} \operatorname{tr}\left(a^{\prime}, 1^{\prime} E\right)=\sum_{E} g_{E} \operatorname{tr}\left(a^{\prime}, E\right)=\tau\left(a^{\prime}\right)
$$

This completes the proof.

\section{The FunCtion $\mathbf{a}_{E}$}

20.1. In this section we assume that the assumptions of 18.1 hold and that $W$ is finite.

The results of $\S 19$ will be applied in the following cases.

(a) $A=\mathcal{H}_{\mathbf{C}}, R=\mathbf{C}$. Here $\mathcal{A} \rightarrow \mathbf{C}$ takes $v$ to 1 . We identify $\mathcal{H}_{\mathbf{C}}$ with the group algebra $\mathbf{C}[W]$ by $w \mapsto T_{w}$ for all $w$. It is well known that $\mathbf{C}[W]$ is a semisimple split algebra. We take $\tau$ so that $\tau(x)=\delta_{x, 1}$ for $x \in W$. Then the bases $(x)$ and $\left(x^{-1}\right)$ are dual with respect to $($,$) .$

We will say " $W$-module" instead of " $\mathbf{C}[W]$-module". We will write $\operatorname{Mod} W, \operatorname{Irr} W$ instead of $\operatorname{Mod} \mathbf{C}[W], \operatorname{Irr} \mathbf{C}[W]$.

(b) $A=J_{\mathbf{C}}, R=\mathbf{C}$. Since $\mathbf{C}[W]$ is semisimple, we see from 18.12 (a) that the kernel of $\phi_{\mathbf{C}}: \mathbf{C}[W] \rightarrow J_{\mathbf{C}}$ is 0 so that $\phi_{\mathbf{C}}$ is injective. Since $\operatorname{dim} \mathbf{C}[W]=\operatorname{dim} J_{\mathbf{C}}=$ $\sharp W$ it follows that $\phi_{\mathbf{C}}$ is an isomorphism. In particular $J_{\mathbf{C}}$ is a semisimple split algebra. We take $\tau: J_{\mathbf{C}} \rightarrow \mathbf{C}$ so that $\tau\left(t_{z}\right)$ is $n_{z}$ if $z \in \mathcal{D}$ and 0 , otherwise. Then $\left(t_{x}, t_{y}\right)=\delta_{x y, 1}$. The bases $\left(t_{x}\right)$ and $\left(t_{x^{-1}}\right)$ are dual with respect to $($,$) .$

(c) $A=\mathcal{H}_{\mathbf{C}(v)}, R=\mathbf{C}(v)$. Here $\mathcal{A} \rightarrow \mathbf{C}$ takes $v$ to $v$. The homomorphism $\phi_{\mathbf{C}(v)}: \mathcal{H}_{\mathbf{C}(v)} \rightarrow J_{\mathbf{C}(v)}$ is injective. This follows from 18.12(b), using the fact that injectivity is preserved by tensoring with a field of fractions. Since $\mathcal{H}_{\mathbf{C}(v)}, J_{\mathbf{C}(v)}$ have the same dimension, it follows that $\phi_{\mathbf{C}(v)}$ is an isomorphism. Since $J_{\mathbf{C}(v)}=$ $\mathbf{C}(v) \otimes J_{\mathbf{C}}$, and $J_{\mathbf{C}}$ is semisimple, split, it follows that $J_{\mathbf{C}(v)}$ is semisimple, split, hence $\mathcal{H}_{\mathbf{C}(v)}$ is semisimple, split. We take $\tau: \mathcal{H}_{\mathbf{C}(v)} \rightarrow \mathbf{C}(v)$ so that $\tau\left(T_{w}\right)=\delta_{w, 1}$. The bases $\left(T_{x}\right)$ and $\left(T_{x^{-1}}\right)$ are dual with respect to $($,$) .$

Remark. The argument above shows also that,

(d) if $R=R_{0}(v)$, with $R_{0}$ an arbitrary field and $\mathcal{A} \rightarrow R$ carries $v$ to $v$, then $\phi_{R}: \mathcal{H}_{R} \rightarrow J_{R}$ is an isomorphism;

(e) if $R$ in 18.11 is a field of characteristic 0 then $\phi_{R}: \mathcal{H}_{R} \rightarrow J_{R}$ is an isomorphism if and only if $\mathcal{H}_{R}$ is a semisimple $R$-algebra.

20.2. For any $E \in \operatorname{Mod} W$ we denote by $E_{\boldsymbol{A}}$ the corresponding $J_{\mathbf{C}}$-module. Thus, $E_{\boldsymbol{\Lambda}}$ coincides with $E$ as a $\mathbf{C}$-vector space and the action of $j \in J_{\mathbf{C}}$ on $E_{\boldsymbol{A}}$ is the

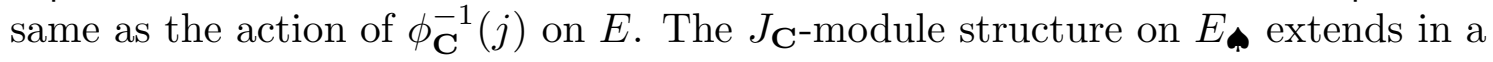


natural way to a $J_{\mathbf{C}(v)}$-module structure on $E_{v}=\mathbf{C}(v) \otimes_{\mathbf{C}} E_{\mathbf{p}}$. We will also regard $E_{v}$ as an $\mathcal{H}_{\mathbf{C}(v)}$-module via the algebra isomorphism $\phi_{\mathbf{C}(v)}: \mathcal{H}_{\mathbf{C}(v)} \stackrel{\sim}{\rightarrow} J_{\mathbf{C}(v)}$. If $E$ is simple, then $E_{\boldsymbol{s}}$ and $E_{v}$ are simple.

Let $E \in \operatorname{Irr} W$. Then $E_{\mathbf{A}}$ is a simple $J_{\mathbf{C}}^{\mathbf{c}}$-module for a unique two-sided cell $\mathbf{c}$ of $W$. Then for any $x \in \mathbf{c}$, we write $E \sim_{\mathcal{L} \mathcal{R}} x$. If $E, E^{\prime} \in \operatorname{Irr} W$, we write $E \sim_{\mathcal{L} \mathcal{R}} E^{\prime}$ if for some $x \in W$ we have $E \sim_{\mathcal{L R}} x, E^{\prime} \sim_{\mathcal{L R}} x$.

20.3. There is the following direct relationship between $E$ and $E_{v}$ (without going through $J)$ :

$$
\operatorname{tr}(x, E)=\left.\operatorname{tr}\left(T_{x}, E_{v}\right)\right|_{v=1} \text { for all } x \in W .
$$

Indeed, it is enough to show that $\operatorname{tr}\left(c_{x}^{\dagger}, E\right)=\left.\operatorname{tr}\left(c_{x}^{\dagger}, E_{v}\right)\right|_{v=1}$. Both sides are equal to $\sum_{z \in W, d \in \mathcal{D}} \gamma_{x, d, z^{-1}} \hat{n}_{z} \operatorname{tr}\left(t_{z}, E_{\boldsymbol{A}}\right)$.

20.4. Assume that $E \in \operatorname{Irr} W$. We have

(a) $\left(f_{E_{v}}\right)_{v=1} \operatorname{dim}(E)=\sharp W$.

Indeed, setting $v=1$ in $\sum_{x \in W} \operatorname{tr}\left(T_{x}, E_{v}\right) \operatorname{tr}\left(T_{x^{-1}}, E_{v}\right)=f_{E_{v}} \operatorname{dim}(E)$ gives

$$
\sum_{x \in W} \operatorname{tr}(x, E) \operatorname{tr}\left(x^{-1}, E\right)=\left(f_{E_{v}}\right)_{v=1} \operatorname{dim}(E)
$$

The left hand side equals $\sharp W$; (a) follows.

20.5. Let $I \subset S$, let $E^{\prime} \in \operatorname{Irr} W_{I}$ and let $E \in \operatorname{Irr} W$. We have

(a) $\left[E_{v}^{\prime}: E_{v}\right]=\left[E^{\prime}: E\right]$.

The right hand side is $\sharp\left(W_{I}\right)^{-1} \sum_{x \in W_{I}} \operatorname{tr}\left(x, E^{\prime}\right) \operatorname{tr}\left(x^{-1}, E\right)$. The left hand side is

$$
f_{E_{v}^{\prime}}^{-1} \operatorname{dim}\left(E^{\prime}\right)^{-1} \sum_{x \in W_{I}} \operatorname{tr}\left(T_{x}, E_{v}^{\prime}\right) \operatorname{tr}\left(T_{x^{-1}}, E_{v}\right)
$$

Since this is a constant, it is equal to its value for $v=1$. Hence it is equal to

$$
\left(f_{E_{v}^{\prime}}^{-1}\right)_{v=1} \operatorname{dim}\left(E^{\prime}\right)^{-1} \sum_{x \in W_{I}} \operatorname{tr}\left(x, E_{v}^{\prime}\right) \operatorname{tr}\left(x^{-1}, E_{v}\right) .
$$

Thus it is enough to show that $\left(f_{E_{v}^{\prime}}\right)_{v=1} \operatorname{dim}\left(E^{\prime}\right)=\sharp\left(W_{I}\right)$. But this is a special case of $20.4(\mathrm{a})$.

Proposition 20.6. Let $E \in \operatorname{Irr} W$.

(a) There exists a unique integer $\mathbf{a}_{E} \geq 0$ such that $\operatorname{tr}\left(T_{x}, E_{v}\right) \in v^{-\mathbf{a}_{E}} \mathbf{C}[v]$ for all $x \in W$ and $\operatorname{tr}\left(T_{x}, E_{v}\right) \notin v^{-\mathbf{a}_{E}+1} \mathbf{C}[v]$ for some $x \in W$.

(b) For $x \in W$ we have $\operatorname{tr}\left(T_{x}, E_{v}\right)=\operatorname{sgn}(x) v^{-\mathbf{a}_{E}} \operatorname{tr}\left(t_{x}, E_{\mathbf{\phi}}\right) \bmod v^{-\mathbf{a}_{E}+1} \mathbf{C}[v]$.

(c) Let $\mathbf{c}$ be the two-sided cell such that $E_{\mathbf{A}} \in \operatorname{Irr} J_{\mathbf{C}}^{\mathbf{c}}$. Then $\mathbf{a}_{E}=\mathbf{a}(x)$ for any $x \in \mathbf{c}$.

Let $a=\mathbf{a}(x)$ for any $x \in \mathbf{c}$. By definition,

$$
\operatorname{tr}\left(c_{x}^{\dagger}, E_{v}\right)=\sum_{z \in W, d \in \mathcal{D} ; \mathbf{a}(d)=\mathbf{a}(z)} h_{x, d, z} \hat{n}_{z} \operatorname{tr}\left(t_{z}, E_{\mathbf{A}}\right) .
$$


In the last sum we have $\operatorname{tr}\left(t_{z}, E_{\boldsymbol{\phi}}\right)=0$ unless $z \in \mathbf{c}$ in which case $\mathbf{a}(z)=a$. For such $z$ we have $h_{x, d, z}=\bar{h}_{x, d, z}=\gamma_{x, d, z^{-1}} v^{-a} \bmod v^{-a+1} \mathbf{Z}[v]$, hence we have

$$
\operatorname{tr}\left(c_{x}^{\dagger}, E_{v}\right)=\sum_{z \in W, d \in \mathcal{D}} \gamma_{x, d, z^{-1}} \hat{n}_{z} \operatorname{tr}\left(t_{z}, E_{\mathbf{\uparrow}}\right) v^{-a} \bmod v^{-a+1} \mathbf{C}[v] .
$$

For each $z$ in the last sum we have $\sum_{d \in \mathcal{D}} \gamma_{x, d, z^{-1}} \hat{n}_{z}=\delta_{x, z} n_{d} \hat{n}_{z}=\delta_{x, z}$. This gives (d) $\operatorname{tr}\left(c_{x}^{\dagger}, E_{v}\right)=\operatorname{tr}\left(t_{x}, E_{\mathbf{\phi}}\right) v^{-a} \bmod v^{-a+1} \mathbf{C}[v]$.

We have $T_{x}=\sum_{y ; y \leq x} q_{y, x}^{\prime} c_{y}$. Hence $\operatorname{sgn}(x) \bar{T}_{x}=T_{x}^{\dagger}=\sum_{y ; y \leq x} q_{y, x}^{\prime} c_{y}^{\dagger}$. Applying ${ }^{-}$ gives $\operatorname{sgn}(x) T_{x}=\sum_{y ; y \leq x} \bar{q}_{y, x}^{\prime} c_{y}^{\dagger}$. Hence

$$
\operatorname{tr}\left(T_{x}, E_{v}\right)=\operatorname{sgn}(x) \sum_{y ; y \leq x} \bar{q}_{y, x}^{\prime} \operatorname{tr}\left(c_{y}^{\dagger}, E_{v}\right) .
$$

Using (d) together with $\bar{q}_{x, x}^{\prime}=1, \bar{q}_{y, x}^{\prime} \in v \mathbf{Z}[v]$ (see 10.1), we deduce

$$
\operatorname{tr}\left(T_{x}, E_{v}\right)=\operatorname{sgn}(x) \operatorname{tr}\left(t_{x}, E_{\mathbf{\phi}}\right) v^{-a} \bmod v^{-a+1} \mathbf{C}[v] .
$$

Since $E_{\boldsymbol{\Lambda}} \in \operatorname{Irr} J_{\mathbf{C}}$, we have $\operatorname{tr}\left(t_{x}, E_{\boldsymbol{\uparrow}}\right) \neq 0$ for some $x \in W$. The proposition follows.

Corollary 20.7. $f_{E_{v}}=f_{E_{\boldsymbol{\omega}}} v^{-2 \mathbf{a}_{E}}+$ strictly higher powers of $v$.

Using 19.2(e) for $\mathcal{H}_{\mathbf{C}(v)}$ and $J_{\mathbf{C}}$, we obtain

$$
\begin{aligned}
& f_{E_{v}} \operatorname{dim} E=\sum_{x} \operatorname{tr}\left(T_{x}, E_{v}\right) \operatorname{tr}\left(T_{x^{-1}}, E_{v}\right) \\
& \in \sum_{x} \operatorname{tr}\left(t_{x}, E_{\boldsymbol{\phi}}\right) \operatorname{tr}\left(t_{x^{-1}}, E_{\boldsymbol{\oplus}}\right) v^{-2 \mathbf{a}_{E}}+v^{-2 \mathbf{a}_{E}+1} \mathbf{C}[v] \\
& =f_{E_{\boldsymbol{\bullet}}} \operatorname{dim} E v^{-2 \mathbf{a}_{E}}+v^{-2 \mathbf{a}_{E}+1} \mathbf{C}[v] .
\end{aligned}
$$

The corollary follows.

Let $^{-}: \mathbf{C}\left[v, v^{-1}\right] \rightarrow \mathbf{C}\left[v, v^{-1}\right]$ be the $\mathbf{C}$-algebra homomorphism given by $v^{n} \mapsto$ $v^{-n}$ for all $n$.

Corollary 20.8. For any $x \in W$ we have $\operatorname{tr}\left(T_{x^{-1}}^{-1}, E_{v}\right)=\overline{\operatorname{tr}\left(T_{x}, E_{v}\right)}$.

We have $T_{x}=\operatorname{sgn}(x) \sum_{y ; y \leq x} \bar{q}_{y, x}^{\prime} c_{y}^{\dagger}, T_{x^{-1}}^{-1}=\operatorname{sgn}(x) \sum_{y ; y \leq x} q_{y, x}^{\prime} c_{y}^{\dagger}$, hence

$$
\begin{aligned}
& \operatorname{tr}\left(T_{x}, E_{v}\right)=\operatorname{sgn}(x) \sum_{y ; y \leq x} \bar{q}_{y, x}^{\prime} \operatorname{tr}\left(c_{y}^{\dagger}, E_{v}\right), \\
& \operatorname{tr}\left(T_{x^{-1}}^{-1}, E_{v}\right)=\operatorname{sgn}(x) \sum_{y ; y \leq x} q_{y, x}^{\prime} \operatorname{tr}\left(c_{y}^{\dagger}, E_{v}\right) .
\end{aligned}
$$

Thus, it suffices to show that $\operatorname{tr}\left(c_{y}^{\dagger}, E_{v}\right)=\overline{\operatorname{tr}\left(c_{y}^{\dagger}, E_{v}\right)}$ for any $y \in W$. As in the proof of 20.6 we have

$$
\operatorname{tr}\left(c_{y}^{\dagger}, E_{v}\right)=\sum_{z \in W, d \in \mathcal{D} ; \mathbf{a}(d)=\mathbf{a}(z)} h_{y, d, z} \hat{n}_{z} \operatorname{tr}\left(t_{z}, E_{\mathbf{⿴}}\right) .
$$

Hence it suffices to show that $\overline{h_{y, d, z}}=h_{y, d, z}$ for all $d, z$ in the last sum. But this is clearly true for any $y, d, z$ in $W$. 
For $E \in \operatorname{Mod} W$ we write $E^{\dagger}, E_{v}^{\dagger}$ instead of $E \otimes \operatorname{sgn},(E \otimes \operatorname{sgn})_{v}$.

Lemma 20.9. Let $E \in \operatorname{Irr} W$. For any $x \in W$ we have $\operatorname{tr}\left(T_{x},\left(E^{\dagger}\right)_{v}\right)=(-1)^{l(x)} \overline{\operatorname{tr}\left(T_{x}, E_{v}\right)}$.

There is a unique a $\mathbf{C}(v)$-algebra involution ${ }^{\dagger}: \mathcal{H}_{\mathbf{C}(v)} \rightarrow \mathcal{H}_{\mathbf{C}(v)}$ extending

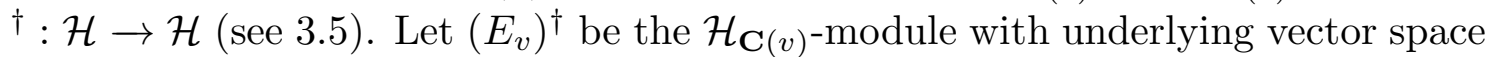
$E_{v}$ such that the action of $h$ on $E_{v}^{\dagger}$ is the same as the action of $h^{\dagger}$ on $E_{v}$. Clearly, $\left(E_{v}\right)^{\dagger} \in \operatorname{Irr}_{\mathbf{C}(v)}$. For $x \in W$ we have

$$
\operatorname{tr}\left(T_{x},\left(E_{v}\right)^{\dagger}\right)=(-1)^{l(x)} \operatorname{tr}\left(T_{x^{-1}}^{-1}, E_{v}\right)=(-1)^{l(x)} \overline{\operatorname{tr}\left(T_{x}, E_{v}\right)}
$$

(The last equation follows from 20.8.) Setting $v=1$ we obtain

$$
\left.\operatorname{tr}\left(T_{x},\left(E_{v}\right)^{\dagger}\right)\right|_{v=1}=(-1)^{l(x)} \operatorname{tr}(x, E)=\operatorname{tr}\left(x, E^{\dagger}\right)
$$

Using 20.3, we deduce that $\left(E_{v}\right)^{\dagger} \cong E_{v}^{\dagger}$ in $\operatorname{Mod} \mathcal{H}_{\mathbf{C}(v)}$. The lemma follows.

Proposition 20.10. For any $x \in W$ we have

$$
\operatorname{tr}\left(T_{x}, E_{v}\right)=\operatorname{tr}\left(t_{x}, E_{\mathbf{\phi}}^{\dagger}\right) v^{\mathbf{a}_{E^{\dagger}}}+\text { strictly lower powers of } v \text {. }
$$

By 20.9 and 20.6 we have

$$
\begin{aligned}
& \operatorname{tr}\left(T_{x}, E_{v}\right)=\operatorname{sgn}(x) \overline{\operatorname{tr}\left(T_{x}, E_{v}^{\dagger}\right)}=\overline{\operatorname{tr}\left(t_{x}, E_{\mathbf{A}}^{\dagger}\right) v^{-\mathbf{a}_{E^{\dagger}}}+\text { strictly higher powers of } v} \\
& =\operatorname{tr}\left(t_{x}, E_{\boldsymbol{\phi}}^{\dagger}\right) v^{\mathbf{a}_{E^{\dagger}}}+\text { strictly lower powers of } v .
\end{aligned}
$$

The proposition is proved.

Corollary 20.11. $f_{E_{v}}=f_{E_{\uparrow}^{\dagger}} v^{2 \mathbf{a}_{E^{\dagger}}}+$ strictly lower powers of $v$.

Using 20.10 we have

$$
\begin{aligned}
& f_{E_{v}} \operatorname{dim} E=\sum_{x} \operatorname{tr}\left(T_{x}, E_{v}\right) \operatorname{tr}\left(T_{x^{-1}}, E_{v}\right) \\
& \in \sum_{x} \operatorname{tr}\left(t_{x}, E_{\mathbf{\uparrow}}^{\dagger}\right) \operatorname{tr}\left(t_{x^{-1}}, E_{\mathbf{\uparrow}}^{\dagger}\right) v^{2 \mathbf{a}_{E^{\dagger}}}+v^{2 \mathbf{a}_{E^{\dagger}}-1} \mathbf{C}\left[v^{-1}\right] \\
& =f_{E_{\boldsymbol{\leftrightarrow}}^{\dagger}} \operatorname{dim} E v^{2 \mathbf{a}_{E^{\dagger}}}+v^{2 \mathbf{a}_{E^{\dagger}}-1} \mathbf{C}\left[v^{-1}\right] .
\end{aligned}
$$

Lemma 20.12. Let $E^{\prime} \in \operatorname{Irr} W_{I}$. We have $g_{E_{v}^{\prime}}=\sum_{E ; E \in \operatorname{Irr} W}\left[E^{\prime}: E\right] g_{E_{v}}$.

We apply 19.4 with $A=\mathcal{H}_{\mathbf{C}(v)}$ and $A^{\prime}$ the analogous algebra for $W_{I}$ instead of $W$, identified naturally with a subspace of $A$. (In this case the unit elements of the two algebras are compatible hence $1_{A^{\prime}} E_{v}=E_{v}$.) It remains to use 20.5(a). 
Lemma 20.13. Let $E \in \operatorname{Irr} W$.

(a) For any $x \in W, \operatorname{tr}\left(t_{x^{-1}}, E_{\boldsymbol{\uparrow}}\right)$ is the complex conjugate of $\operatorname{tr}\left(t_{x}, E_{\boldsymbol{\uparrow}}\right)$.

(b) $f_{E_{\star}}$ is a strictly positive real number.

We prove (a). Let $\langle\rangle:, E \times E \rightarrow \mathbf{C}$ be a positive definite hermitian form. We define $\langle,\rangle^{\prime}: E_{\boldsymbol{\phi}} \times E_{\boldsymbol{\phi}} \rightarrow \mathbf{C}$ by $\left\langle e, e^{\prime}\right\rangle^{\prime}=\sum_{z \in W}\left\langle t_{z} e, t_{z} e^{\prime}\right\rangle$. This is again a positive definite hermitian form on $E_{\boldsymbol{A}}$. We show that

$$
\left\langle t_{x} e, e^{\prime}\right\rangle^{\prime}=\left\langle e, t_{x^{-1}} e^{\prime}\right\rangle^{\prime}
$$

for all $e, e^{\prime}$. This is equivalent to

$$
\sum_{y, z} \gamma_{z, x, y^{-1}}\left\langle t_{y} e, t_{z} e^{\prime}\right\rangle=\sum_{y, z} \gamma_{y, x^{-1}, z^{-1}}\left\langle t_{y} e, t_{z} e^{\prime}\right\rangle
$$

which follows from $\gamma_{z, x, y^{-1}}=\gamma_{y, x^{-1}, z^{-1}}$. We see that $t_{x^{-1}}$ is the adjoint of $t_{x}$ with respect to a positive definite hermitian form. (a) follows.

We prove (b). By 19.2(e) we have $f_{E_{\boldsymbol{\Lambda}}} \operatorname{dim}(E)=\sum_{x} \operatorname{tr}\left(t_{x}, E_{\boldsymbol{\mathbf { A }}}\right) \operatorname{tr}\left(t_{x^{-1}}, E_{\mathbf{\phi}}\right)$. The right hand side of this equality is a real number $\geq 0$, by (a). Hence so is the left hand side. Now $f_{E_{\boldsymbol{\omega}}} \neq 0$ by $19.2(\mathrm{~d})$ and (b) follows.

Proposition 20.14. Let $E^{\prime} \in \operatorname{Irr} W_{I}$.

(a) For any $E \in \operatorname{Irr} W$ such that $\left[E^{\prime}: E\right] \neq 0$ we have $\mathbf{a}_{E^{\prime}} \leq \mathbf{a}_{E}$.

(b) We have $g_{E_{\boldsymbol{\omega}}^{\prime}}=\sum\left[E^{\prime}: E\right] g_{E_{\boldsymbol{\omega}}}$, sum over all $E \in \operatorname{Irr} W$ (up to isomorphism) such that $\mathbf{a}_{E}=\mathbf{a}_{E^{\prime}}$.

Let $X$ be the set of all $E$ (up to isomorphism) such that $\left[E^{\prime}: E\right] \neq 0$ and such that $\mathbf{a}_{E}$ is minimum, say equal to $a$. Assume first that $a<\mathbf{a}_{E^{\prime}}$. Using 19.2(d) we rewrite 20.12 in the form

(c) $v^{-2 a} f_{E_{v}^{\prime}}^{-1}=\sum_{E}\left[E^{\prime}: E\right] v^{-2 a} f_{E_{v}}^{-1}$.

By 20.7, we have

(d) $\left.\left(v^{-2 \mathbf{a}_{E}} f_{E_{v}}^{-1}\right)\right|_{v=0}=f_{E_{\boldsymbol{\leftrightarrow}}}^{-1},\left.\left(v^{-2 \mathbf{a}_{E^{\prime}}} f_{E_{v}^{\prime}}^{-1}\right)\right|_{v=0}=f_{E_{\boldsymbol{\phi}}^{\prime}}^{-1}$,

hence by setting $v=0$ in (c) we obtain

$$
0=\sum_{E \in X}\left[E^{\prime}: E\right] f_{E_{\bullet}}^{-1}
$$

The right hand side is a real number $>0$ by 20.8 (b). This is a contradiction. Thus we must have $a \geq \mathbf{a}_{E^{\prime}}$ and (a) is proved.

We now rewrite (c) in the form

(e) $v^{-2 \mathbf{a}_{E^{\prime}}} f_{E_{v}^{\prime}}^{-1}=\sum_{E}\left[E^{\prime}: E\right] v^{-2 \mathbf{a}_{E^{\prime}}} f_{E_{v}}^{-1}$.

Using (d) and (a) we see that, setting $v=0$ in (e) gives

$$
f_{E_{\boldsymbol{a}}^{\prime}}^{-1}=\sum_{E ; \mathbf{a}_{E}=\mathbf{a}_{E^{\prime}}}\left[E^{\prime}: E\right] f_{E_{\mathbf{\bullet}}}^{-1}
$$

This proves (b). 
20.15. Let $K(W)$ be the $\mathbf{C}$-vector space with basis indexed by the $E \in \operatorname{Irr} W$ (up to isomorphism). If $\tilde{E} \in \operatorname{Mod} W$ we identify $\tilde{E}$ with the element $\sum_{E}[E: \tilde{E}] E \in$ $K(W)$ ( $E$ as above). We define a C-linear map $\mathbf{j}_{W_{I}}^{W}: K\left(W_{I}\right) \rightarrow K(W)$ by

$\mathbf{j}_{W}^{W}\left(E^{\prime}\right)=\sum_{E}\left[E^{\prime}: E\right] E$

sum over all $E \in \operatorname{Irr} W$ (up to isomorphism) such that $\mathbf{a}_{E}=\mathbf{a}_{E^{\prime}}$; here $E^{\prime} \in \operatorname{Irr} W_{I}$.

We call this truncated induction.

Let $I^{\prime \prime} \subset I^{\prime} \subset S$. We show that the following transitivity formula holds:

$$
\mathbf{j}_{W_{I^{\prime}}}^{W} \mathbf{j}_{W_{I^{\prime \prime}}}^{W_{I^{\prime}}}=\mathbf{j}_{W_{I^{\prime \prime}}}^{W}: K\left(W_{I^{\prime \prime}}\right) \rightarrow K(W)
$$

Let $E^{\prime \prime} \in \operatorname{Irr} W_{I^{\prime \prime}}$. We must show that

$$
\left[E^{\prime \prime}: E\right]=\sum_{E^{\prime} ; \mathbf{a}_{E^{\prime}}=\mathbf{a}_{E^{\prime \prime}}}\left[E^{\prime \prime}: E^{\prime}\right]\left[E^{\prime}: E\right]
$$

for any $E^{\prime \prime} \in \operatorname{Irr} W_{I^{\prime \prime}}, E \in \operatorname{Irr} W$ such that $\mathbf{a}_{E^{\prime \prime}}=\mathbf{a}_{E}$; in the sum we have $E^{\prime} \in$ $\operatorname{Irr} W_{I^{\prime}}$. Clearly,

$$
\left[E^{\prime \prime}: E\right]=\sum_{E^{\prime}}\left[E^{\prime \prime}: E^{\prime}\right]\left[E^{\prime}: E\right]
$$

Hence it is enough to show that, if $\left[E^{\prime \prime}: E^{\prime}\right]\left[E^{\prime}: E\right] \neq 0$, then we automatically have $\mathbf{a}_{E^{\prime}}=\mathbf{a}_{E^{\prime \prime}}$. By 2.10(a) we have $\mathbf{a}_{E^{\prime \prime}} \leq \mathbf{a}_{E^{\prime}} \leq \mathbf{a}_{E}$. Since $\mathbf{a}_{E^{\prime \prime}}=\mathbf{a}_{E}$, the desired conclusion follows.

20.16. For any $x \in W$ we set

$$
\gamma_{x}=\sum_{E ; E \in \operatorname{Irr} W} \operatorname{tr}\left(t_{x}, E_{\mathbf{\uparrow}}\right) E \in K(W)
$$

We sometimes write $\gamma_{x}^{W}$ instead of $\gamma_{x}$, to emphasize dependence on $W$. Note that $\gamma_{x}$ is a C-linear combination of $E$ such that $E \sim_{\mathcal{L} \mathcal{R}} x$. Hence, if $E, E^{\prime}$ appear with $\neq 0$ coefficient in $\gamma_{x}$ then $E \sim_{\mathcal{L} \mathcal{R}} E^{\prime}$.

Proposition 20.17. If $x \in W_{I}$, then $\gamma_{x}^{W}=\mathbf{j}_{W_{I}}^{W}\left(\gamma_{x}^{W_{I}}\right)$.

An equivalent statement is

$$
\operatorname{tr}\left(t_{x}, E_{\mathbf{\phi}}\right)=\sum_{E^{\prime} ; \mathbf{a}_{E}=\mathbf{a}_{E^{\prime}}} \operatorname{tr}\left(t_{x}, E_{\mathbf{\phi}}^{\prime}\right)\left[E^{\prime}: E\right]
$$

for any $E \in \operatorname{Irr} W$; in the sum we have $E^{\prime} \in \operatorname{Irr} W_{I}$. Clearly, we have

$$
v^{\mathbf{a}_{E}} \operatorname{tr}\left(T_{x}, E_{v}\right)=\sum_{E^{\prime} ; E^{\prime} \in \operatorname{Irr} W_{I}} v^{\mathbf{a}_{E}} \operatorname{tr}\left(T_{x}, E_{v}^{\prime}\right)\left[E^{\prime}: E\right] .
$$

In the right hand side we may assume that $\mathbf{a}_{E^{\prime}} \leq \mathbf{a}_{E}$. Using this and 20.6, we see that setting $v=0$ in (b) gives (a). The proposition is proved. 
Lemma 20.18. (a) We have $\mathbf{a}_{\mathrm{sgn}}=L\left(w_{0}\right)$.

(b) We have $f_{\operatorname{sgn}_{\star}}=1$.

(c) We have $\gamma_{w_{0}}=\operatorname{sgn}$.

$\operatorname{sgn}_{v}$ is the one dimensional $\mathcal{H}_{\mathbf{C}(v)}$-module on which $T_{x}$ acts as $\operatorname{sgn}(x) v^{-L(x)}$. (This follows from 20.3.) From 20.6(b) we see that $\mathbf{a}_{\mathrm{sgn}}=L\left(w_{0}\right)$ and that $\operatorname{tr}\left(t_{w_{0}}, \operatorname{sgn} \mathbf{\infty}\right)=1$. This proves (a). To prove (c) it remains to show that, if $\operatorname{tr}\left(t_{w_{0}}, E_{\mathbf{A}}\right) \neq 0$ (E simple) then $E \cong \operatorname{sgn}$. This assumption shows, by 20.6(c), that $E_{\boldsymbol{s}} \in \operatorname{Irr} J_{\mathbf{C}}^{\mathbf{c}}$ where $\mathbf{c}$ is the two-sided cell such that $\operatorname{sgn} \boldsymbol{\Phi}_{\boldsymbol{\phi}} \in \operatorname{Irr} J_{\mathbf{C}}^{\mathbf{c}}$. Since $\operatorname{tr}\left(t_{w_{0}}, \operatorname{sgn} \mathbf{\infty}\right)=1$, we have $w_{0} \in \mathbf{c}$. From 13.8 it follows that $\left\{w_{0}\right\}$ is a two-sided cell. Thus $\mathbf{c}=\left\{w_{0}\right\}$ and $J_{\mathbf{C}}^{\mathbf{c}}$ is one dimensional. Hence it cannot have more than one simple module. Thus, $E \cong \operatorname{sgn}$. This yields (c) and also (b). The lemma is proved.

20.19. Assume that $I, I^{\prime}$ form a partition of $S$ such that $W=W_{I} \times W_{I^{\prime}}$. If $E \in \operatorname{Irr} W_{I}$ and $E^{\prime} \in \operatorname{Irr} W_{I^{\prime}}$, then $E \otimes E^{\prime} \in \operatorname{Irr} W$. From the definitions,

$$
\mathbf{a}_{E \otimes E^{\prime}}=\mathbf{a}_{E}+\mathbf{a}_{E^{\prime}}, f_{\left(E \otimes E^{\prime}\right)}=f_{E_{\boldsymbol{\omega}}} f_{E_{\boldsymbol{\omega}}^{\prime}} .
$$

Moreover, if $x \in W_{I}, x^{\prime} \in W_{I^{\prime}}$, then

$$
\gamma_{x x^{\prime}}^{W}=\gamma_{x}^{W_{I}} \otimes \gamma_{x^{\prime}}^{W_{I^{\prime}}}
$$

20.20. In the remainder of this section we assume that $w_{0}$ is in the centre of $W$. Then, for any $E \in \operatorname{Irr} W, w_{0}$ acts on $E$ as $\epsilon_{E}$ times identity where $\epsilon_{E}= \pm 1$. Now $E \mapsto \epsilon_{E} E$ extends to a C-linear involution $\zeta: K(W) \rightarrow K(W)$.

Lemma 20.21. Let $E \in \operatorname{Irr} W$. For any $x \in W$ we have

$$
\operatorname{tr}\left(T_{w_{0} x}, E_{v}\right)=\epsilon_{E} v^{-\mathbf{a}_{E}+\mathbf{a}_{E^{\dagger}} \overline{\operatorname{tr}\left(T_{x}, E_{v}\right)}} \text {. }
$$

Since $w_{0}$ is in the centre of $W, T_{w_{0}}$ is in the centre of $\mathcal{H}_{\mathbf{C}(v)}$ hence it acts on $E_{v}$ as a scalar $\lambda \in \mathbf{C}(v)$ times the identity. Now $\operatorname{tr}\left(T_{x}, E_{v}\right) \in \mathbf{C}\left[v, v^{-1}\right]$ and $\operatorname{tr}\left(T_{x}^{-1}, E_{v}\right) \in \mathbf{C}\left[v, v^{-1}\right]$. In particular, $\lambda \in \mathbf{C}\left[v, v^{-1}\right]$ and $\lambda^{-1} \in \mathbf{C}\left[v, v^{-1}\right]$. This implies $\lambda=c v^{n}$ where $c \in \mathbf{C}$. For $v=1, \lambda$ becomes $\epsilon_{E}$. Hence $\lambda=\epsilon_{E} v^{n}$ for some $n$. We have

$$
\operatorname{tr}\left(T_{w_{0} x}, E_{v}\right)=\operatorname{tr}\left(T_{w_{0}} T_{x^{-1}}^{-1}, E_{v}\right)=\lambda \operatorname{tr}\left(T_{x^{-1}}^{-1}, E_{v}\right)=\lambda \overline{\operatorname{tr}\left(T_{x}, E_{v}\right)} .
$$

We have

$$
\sum_{x} \operatorname{tr}\left(T_{w_{0} x}, E_{v}\right) \operatorname{tr}\left(T_{x^{-1} w_{0}}, E_{v}\right)=\lambda^{2} \sum_{x} \overline{\operatorname{tr}\left(T_{x}, E_{v}\right) \operatorname{tr}\left(T_{x^{-1}}, E_{v}\right)}
$$

hence $f_{E_{v}} \operatorname{dim}(E)=\lambda^{2} \overline{f_{E_{v}}} \operatorname{dim}(E)$ so that $f_{E_{v}}=v^{2 n} \overline{f_{E_{v}}}$. By 20.9, we have

$$
\sum_{x} \overline{\operatorname{tr}\left(T_{x}, E_{v}\right) \operatorname{tr}\left(T_{x^{-1}}, E_{v}\right)}=\sum_{x} \operatorname{tr}\left(T_{x}, E_{v}^{\dagger}\right) \operatorname{tr}\left(T_{x^{-1}}, E_{v}^{\dagger}\right)
$$

hence $\overline{f_{E_{v}}}=f_{E_{v}^{\dagger}}$. We see that $f_{E_{v}}=v^{2 n} f_{\left(E^{\dagger}\right)_{v}}$. Comparing the lowest terms we see that $-2 \mathbf{a}_{E}=2 n-2 \mathbf{a}_{E^{\dagger}}$ hence $n=-\mathbf{a}_{E}+\mathbf{a}_{E^{\dagger}}$ and that

(a) $f_{E_{\boldsymbol{\omega}}}=f_{E_{\boldsymbol{\phi}}^{\dagger}}$. 
Lemma 20.22. $v^{\mathbf{a}_{E}} \operatorname{tr}\left(T_{w_{0} x}, E_{v}\right)=\epsilon_{E}(-1)^{l(x)} v^{\mathbf{a}_{E} \dagger} \operatorname{tr}\left(T_{x}, E_{v}^{\dagger}\right)$.

We combine 20.8, 20.21.

Lemma 20.23. For any $x \in W$ we have $\gamma_{x w_{0}}=\operatorname{sgn}(x) \zeta\left(\gamma_{x}\right) \otimes \operatorname{sgn}$.

An equivalent statement is

$$
\operatorname{tr}\left(t_{x w_{0}}, E_{\mathbf{\uparrow}}\right)=\operatorname{sgn}(x) \operatorname{tr}\left(t_{x}, E_{\mathbf{\phi}}^{\dagger}\right) \epsilon_{E^{\dagger}}
$$

for any $E \in \operatorname{Irr} W$. Setting $v=0$ in the identity in 20.22 gives

$$
\operatorname{sgn}\left(x w_{0}\right) \operatorname{tr}\left(t_{w_{0} x}, E_{\mathbf{\phi}}\right)=\epsilon_{E} \operatorname{tr}\left(t_{x}, E_{\mathbf{\phi}}^{\dagger}\right) .
$$

It remains to show that $\epsilon_{E^{\dagger}}=\epsilon_{E} \operatorname{sgn}\left(w_{0}\right)$. This is clear.

20.24. By the Cayley-Hamilton theorem, any element $r \in J$ satisfies an equation of the form $r^{n}+a_{1} r^{n-1}+\cdots+a_{n}=0$ where $a_{i} \in \mathbf{Z}$. (We use that the structure constants of $J$ are integers.) This holds in particular for $r=t_{x}$ where $x \in W$. Hence for any $\mathcal{E} \in \operatorname{Irr} J_{\mathbf{C}}, \operatorname{tr}\left(t_{x}, \mathcal{E}\right)$ is an algebraic integer. If $R$ is a subfield of $\mathbf{C}$ such that the group algebra $R[W]$ is split over $R$, then $J_{R}$ is split over $R$ and it follows that for $x, \mathcal{E}$ as above, $\operatorname{tr}\left(t_{x}, \mathcal{E}\right)$ is an algebraic integer in $R$. In particular, if we can take $R=\mathbf{Q}$, then $\operatorname{tr}\left(t_{x}, \mathcal{E}\right) \in \mathbf{Z}$.

\section{STUDY OF A LEFT CELL}

21.1. In this section we preserve the setup of 20.1. Let $\Gamma$ be a left cell of $W, L$. Let $d$ be the unique element in $\Gamma \cap \mathcal{D}$. The $\mathcal{A}$-submodule $\sum_{y \in \Gamma} \mathcal{A} c_{y}^{\dagger}$ of $\mathcal{H}$ can be regarded as an $\mathcal{H}$-module by the rule $c_{x}^{\dagger} \cdot c_{w}^{\dagger}=\sum_{z \in \Gamma} h_{x, y, z} c_{z}^{\dagger}$ with $x \in W, y \in W$. By change of scalars $(v \mapsto 1)$ this gives rise to an $\mathcal{H}_{\mathbf{C}}=\mathbf{C}[W]$-module $[\Gamma]$. On the other hand, $J_{\mathbf{C}}^{\Gamma}=\bigoplus_{y \in \Gamma} \mathbf{C} t_{y}$ is a left ideal in $J_{\mathbf{C}}$ by $14.2(\mathrm{P} 8)$.

Lemma 21.2. The C-linear isomorphism $t_{y} \mapsto \hat{n}_{y} c_{y}^{\dagger}$ for $y \in \Gamma$ is an isomorphism of $J_{\mathbf{C}}$-modules $J_{\mathbf{C}}^{\Gamma} \stackrel{\sim}{\longrightarrow}[\Gamma]_{\mathbf{p}}$.

We have $\Gamma \subset X_{a}=\{w \in W ; \mathbf{a}(x)=a\}$ for some $a \in \mathbf{N}$. The $\mathcal{A}$-submodule $\sum_{y \in X_{a}} \mathcal{A} c_{y}^{\dagger}$ of $\mathcal{H}$ can be regarded as an $\mathcal{H}$-module by the rule

$$
c_{x}^{\dagger} \cdot c_{w}^{\dagger}=\sum_{z \in X_{a}} h_{x, y, z} c_{z}^{\dagger}
$$

with $x \in W, y \in W$. By change of scalars $(v \mapsto 1)$ this gives rise to an $\mathcal{H}_{\mathbf{C}}=\mathbf{C}[W]$ module $\left[X_{a}\right]$. On the other hand, $J_{\mathbf{C}}^{X_{a}}=\oplus_{y \in X_{a}} \mathbf{C} t_{y}$ is a left (even two-sided) ideal in $J_{\mathbf{C}}$. The $\mathbf{C}$-linear map in the lemma extends by the same formula to a $\mathbf{C}$-linear isomorphism $J_{\mathbf{C}}^{X_{a}} \stackrel{\sim}{\longrightarrow}\left[X_{a}\right]_{\mathbf{p}}$. It is enough to show that this is $J_{\mathbf{C}}$-linear. This follows from the computation in 18.10. The lemma is proved. 
Lemma 21.3. Let $\mathcal{E} \in \operatorname{Irr} J_{\mathbf{C}}$. The $\mathbf{C}$-linear map $u: \operatorname{Hom}_{J_{\mathbf{C}}}\left(J_{\mathbf{C}}^{\Gamma}, \mathcal{E}\right) \rightarrow t_{d} \mathcal{E}$ given by $\xi \mapsto \xi\left(n_{d} t_{d}\right)$ is an isomorphism.

$u$ is well defined since $\xi\left(n_{d} t_{d}\right)=t_{d} \xi\left(t_{d}\right) \in t_{d} \mathcal{E}$. We define a linear map in the opposite direction by $e \mapsto[j \mapsto j e]$. It is clear that this is the inverse of $u$. (We use that $j n_{d} t_{d}=j$ for $j \in J_{\mathbf{C}}^{\Gamma}$.) The lemma is proved.

Proposition 21.4. We have $\gamma_{d}=n_{d} \sum_{E}[E:[\Gamma]] E$ (sum over all $E \in \operatorname{Irr} W$ up to isomorphism).

An equivalent statement is that $\operatorname{tr}\left(n_{d} t_{d}, E_{\boldsymbol{A}}\right)=[E:[\Gamma]]$, for $E$ as above. By 21.2 , we have $[E:[\Gamma]]=\left[E_{\boldsymbol{\omega}}: J_{\mathbf{C}}^{\Gamma}\right]$. Hence it remains to show that $\operatorname{tr}\left(n_{d} t_{d}, \mathcal{E}\right)=$ $\left[\mathcal{E}: J_{\mathbf{C}}^{\Gamma}\right]$ for any $\mathcal{E} \in \operatorname{Irr} J_{\mathbf{C}}$. Since $\mathcal{E}=\oplus_{d^{\prime} \in \mathcal{D}} n_{d^{\prime}} t_{d^{\prime}} \mathcal{E}$ and $n_{d} t_{d}: \mathcal{E} \rightarrow \mathcal{E}$ is the projection to the summand $n_{d} t_{d} \mathcal{E}$, we see that $\operatorname{tr}\left(n_{d} t_{d}, \mathcal{E}\right)=\operatorname{dim}\left(t_{d} \mathcal{E}\right)$. It remains to show that $\operatorname{dim}\left(t_{d} \mathcal{E}\right)=\left[\mathcal{E}: J_{\mathbf{C}}^{\Gamma}\right]$. This follows from 21.3.

Proposition 21.5. $[\Gamma]^{\dagger},\left[\Gamma w_{0}\right]$ are isomorphic in $\operatorname{Mod} W$.

We may identify $[\Gamma]^{\dagger}$ with the $W$-module with $\mathbf{C}$-basis $\left(e_{y}\right)_{y \in \Gamma}$ where $s \in S$ acts by $e_{y} \mapsto-e_{y}+\sum_{z \in \Gamma} h_{s, y, z} e_{z}$.

On the other hand we may identify $\left[\Gamma w_{0}\right]$ with the $W$-module with C-basis $\left(e_{y w_{0}}^{\prime}\right)_{y \in \Gamma}$ where $s \in S$ acts by $e_{y w_{0}}^{\prime} \mapsto e_{y w_{0}}^{\prime}-\sum_{z \in \Gamma} h_{s, y w_{0}, z w_{0}} e_{z w_{0}}^{\prime}$.

The $W$-module dual to $[\Gamma]^{\dagger}$ has a $\mathbf{C}$-basis $\left(e_{y}^{\prime \prime}\right)_{y \in \Gamma}\left(\right.$ dual to $\left.\left(e_{y}\right)\right)$ in which the action of $s \in S$ is given by $e_{y}^{\prime \prime} \mapsto-e_{y}^{\prime \prime}+\sum_{z \in \Gamma} h_{s, z, y} e_{z}^{\prime \prime}$. We define a C-isomorphism between this last space and $\left[\Gamma w_{0}\right]$ by $e_{y}^{\prime \prime} \mapsto \operatorname{sgn}(y) e_{y w_{0}}^{\prime}$ for all $y$. We show that this comutes with the action of $W$. It suffices to show that for any $s \in S$, we have

(a) $-h_{s, z, y}=\operatorname{sgn}(y) \operatorname{sgn}(z) h_{s, y w_{0}, z w_{0}}$ for all $z \neq y$ and

(b) $1-h_{s, y, y}=-1+h_{s, y w_{0}, y w_{0}}$ for all $y$.

We use 6.6. Assume first that $s z>z$. If $s y>y$ and $y \neq z$, both sides of (a) are 0 . If $s y<y<z$ then (a) follows from 11.6. If $y=s z$ then both sides of (a) are -1 . If $s y<y$ but $y \nless z$ or $y \neq s z$ then both sides of (a) are 0 .

Assume next that $s z<z$. If $z \neq y$ then both sides of (a) are 0 .

If $s y>y$, both sides of (b) are 1 . If $s y<y$, both sides of (b) are -1 . Thus (a),(b) are verified. Since $[\Gamma]^{\dagger}$ and its dual are isomorphic in $\operatorname{Mod} W$ (they are defined over $\mathbf{Q}$ ), the lemma follows.

Corollary 21.6. Let $E \in \operatorname{Irr} W$ and let $\mathbf{c}$ be the two-sided cell of $W$ such that $E_{\boldsymbol{\phi}} \in \operatorname{Irr} J_{\mathbf{C}}^{\mathbf{c}}$. Then $E_{\boldsymbol{\phi}}^{\dagger} \in \operatorname{Irr} J_{\mathbf{C}}^{\mathbf{c} w_{0}}$.

Replacing $\Gamma$ by $\mathbf{c}$ in the definition of $[\Gamma]$ we obtain a $W$-module $[\mathbf{c}]$. Then 21.2, 21.5 hold with $\Gamma$ replaced by $\mathbf{c}$ with the same proof. Our assumption implies (by 21.2 for $\mathbf{c}$ ) that $E$ appears in the $W$-module $[\mathbf{c}]$. Using 21.5 for $\mathbf{c}$ we deduce that $E^{\dagger}$ appears in the $W$-module $\left[\mathbf{c} w_{0}\right]$. Using 21.2 for $\mathbf{c} w_{0}$, we deduce that $E_{\mathbf{A}}^{\dagger}$

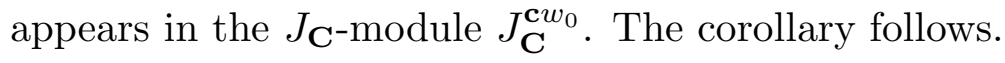

Corollary 21.7. Let $E, E^{\prime} \in \operatorname{Irr} W$ be such that $E \sim_{\mathcal{L R}} E^{\prime}$. Then $E^{\dagger} \sim_{\mathcal{L R}} E^{\prime \dagger}$. 
By assumption there exists a two-sided cell $\mathbf{c}$ such that $E_{\mathbf{A}}, E_{\mathbf{A}}^{\prime} \in \operatorname{Irr} J_{\mathbf{C}}^{\mathbf{c}}$. By 21.6, $E_{\mathbf{A}}^{\dagger}, E^{\prime \dagger} \in \operatorname{Irr} J_{\mathbf{C}}^{\mathbf{c} w_{0}}$. The corollary follows.

21.8. The results of $\S 19$ are applicable to $A$, the $\mathbf{C}$-subspace $J_{\mathbf{C}}^{\Gamma \cap \Gamma^{-1}}$ of $J_{\mathbf{C}}$ spanned by $\Gamma \cap \Gamma^{-1}$ and $R=\mathbf{C}$. This is a $\mathbf{C}$-subalgebra of $J_{\mathbf{C}}$ with unit element $n_{d} t_{d}$. In 21.9 we will show that $J_{\mathbf{C}}^{\Gamma \cap \Gamma^{-1}}$ is semisimple. It is then clearly split. We take $\tau: J_{\mathbf{C}}^{\Gamma \cap \Gamma^{-1}} \rightarrow \mathbf{C}$ so that $\tau\left(t_{x}\right)=n_{d} \delta_{x, d}$. (This is the restriction of $\tau: J_{\mathbf{C}} \rightarrow \mathbf{C}$.) We have $\left(t_{x}, t_{y}\right)=\delta_{x y, 1}$. The bases $\left(t_{x}\right)$ and $\left(t_{x^{-1}}\right)$ (where $x$ runs through $\Gamma \cap \Gamma^{-1}$ ) are dual with respect to $($,$) .$

21.9. We show that the $\mathbf{C}$-algebra $J_{\mathbf{C}}^{\Gamma \cap \Gamma^{-1}}$ is semisimple. It is enough to prove the analogous statement for the Q-algebra $A^{\prime}$, the $\mathbf{Q}$-span of $\Gamma \cap \Gamma^{-1}$ in $J_{\mathbf{Q}}$. We define a Q-bilinear pairing $(\mid): A^{\prime} \times A^{\prime} \rightarrow \mathbf{Q}$ by $\left(t_{x} \mid t_{y}\right)=\delta_{x, y}$ for $x, y \in \Gamma \cap \Gamma^{-1}$. Let $j \mapsto \tilde{j}$ be the Q-linear map $A^{\prime} \rightarrow A^{\prime}$ given by $\tilde{t}_{x}=t_{x^{-1}}$ for all $x$. We show that

$$
\left(j_{1} j_{2} \mid j_{3}\right)=\left(j_{2} \mid \tilde{j}_{1} j_{3}\right)
$$

for all $j_{1}, j_{2}, j_{3}$ in our ring. We may assume that $j_{1}=t_{x}, j_{2}=t_{y}, j_{3}=t_{z}$. Then (a) follows from

$$
\gamma_{x, y, z^{-1}}=\gamma_{x^{-1}, z, y^{-1}}
$$

Now let $I$ be a left ideal of $A^{\prime}$. Let $I^{\perp}=\left\{a \in A^{\prime} ;(a \mid I)=0\right\}$. Since $(\mid)$ is positive definite, we have $A^{\prime}=I \oplus I^{\perp}$. From (c) we see that $I^{\perp}$ is a left ideal. This proves that $A^{\prime}$ is semisimple.

The same proof could be used to show directly that $J_{\mathbf{C}}$ is semisimple.

Proposition 21.10. Let $E, E^{\prime} \in \operatorname{Irr} W, N=\sum_{x \in \Gamma^{-1}} \operatorname{tr}\left(t_{x}, E_{\mathbf{\phi}}\right) \operatorname{tr}\left(t_{x^{-1}}, E_{\mathbf{\phi}}^{\prime}\right)$. Then $N=f_{E_{\boldsymbol{~}}}[E:[\Gamma]]$ if $E, E^{\prime}$ are isomorphic and $N=0$, otherwise.

If $\mathcal{E} \in \operatorname{Irr} J_{\mathbf{C}}$, then $t_{d} \mathcal{E}$ is either 0 or in $\operatorname{Irr} J_{\mathbf{C}}^{\Gamma \cap \Gamma^{-1}}$. Moreover, $\mathcal{E} \mapsto t_{d} \mathcal{E}$ defines a bijection between the set of simple $J_{\mathbf{C}}$-modules (up to isomorphism) which appear in the $J_{\mathbf{C}}$-module $J_{\mathbf{C}}^{\Gamma}$ and the set of simple $J_{\mathbf{C}}^{\Gamma \cap \Gamma^{-1}}$-modules (up to isomorphism). We then have $\operatorname{dim}\left(t_{d} \mathcal{E}\right)=\left[\mathcal{E}: J_{\mathbf{C}}^{\Gamma}\right]$. For $j \in J_{\mathbf{C}}^{\Gamma \cap \Gamma^{-1}}$ we have $\operatorname{tr}(j, \mathcal{E})=\operatorname{tr}\left(j, t_{d} \mathcal{E}\right)$. If $t_{d} E_{\boldsymbol{\phi}}=0$ or $t_{d} E_{\boldsymbol{\Delta}}^{\prime}=0$, then $N=0$ and the result is clear. If $t_{d} E_{\boldsymbol{\phi}} \neq 0$ and $t_{d}^{\prime} E_{\boldsymbol{\phi}} \neq 0$ then, by $19.2(\mathrm{e})$, we see that $N=f_{t_{d} E_{\mathbf{A}}}\left[E_{\boldsymbol{\phi}}: J_{\mathbf{C}}^{\Gamma}\right]$ if $E, E^{\prime}$ are isomorphic and to 0, otherwise. It remains to show that $f_{t_{d} E_{\boldsymbol{\omega}}}=f_{E_{\boldsymbol{\omega}}},[E:[\Gamma]]=\left[E_{\boldsymbol{\phi}}: J_{\mathbf{C}}^{\Gamma}\right]$ and the analogous equalities for $E^{\prime}$. Now $f_{t_{d} E_{\boldsymbol{\Lambda}}}=f_{E_{\boldsymbol{\omega}}}$ follows from 19.4 applied to $\left(A^{\prime}, A\right)=\left(J_{\mathbf{C}}^{\Gamma \cap \Gamma^{-1}}, J_{\mathbf{C}}\right)$; the equality $[E:[\Gamma]]=\left[E_{\boldsymbol{\phi}}: J_{\mathbf{C}}^{\Gamma}\right]$ follows from 21.2. The proposition is proved.

\section{Constructible Representations}

22.1. In this section we preserve the setup of 20.1 . 
We define a class $\operatorname{Con}(W)$ of $W$-modules (relative to our fixed $L: W \rightarrow \mathbf{N}$ ) by induction on $\sharp S$. If $\sharp S=0$ so that $W=\{1\}$, $\operatorname{Con}(W)$ consists of the unit representation. Assume now that $\sharp S>0$. Then Con $(W)$ consists of the $W$ modules of the form $\mathbf{j}_{W_{I}}^{W}\left(E^{\prime}\right)$ or $\mathbf{j}_{W_{I}}^{W}\left(E^{\prime}\right) \otimes \operatorname{sgn}$ for various subsets $I \subset S, I \neq S$ and various $E^{\prime} \in \operatorname{Con}\left(W_{I}\right)$. (If we restrict ourselves to $I$ such that $\sharp(S-I)=1$ we get the same class of $W$-modules, by the transitivity of truncated induction.) The $W$-modules in Con $(W)$ are said to be the constructible representations of $W$.

Now the unit representation of $W$ is constructible (it is obtained by truncated induction from the unit representation of the subgroup with one element). Hence $\operatorname{sgn} \in \operatorname{Con}(W)$.

Lemma 22.2. If $E \in \operatorname{Con}(W)$, then there exists a left cell $\Gamma$ of $W$ such that $E=[\Gamma]$.

We argue by induction on $\sharp S$. If $\sharp S=0$ the result is obvious. Assume now that $\sharp S>0$. Let $E \in \operatorname{Con}(W)$.

Case 1. $E=\mathbf{j}_{W_{I}}^{W}\left(E^{\prime}\right)$ where $I \subset S, I \neq S$ and $E^{\prime} \in \operatorname{Con}\left(W_{I}\right)$. By the induction hypothesis there exists a left cell $\Gamma^{\prime}$ of $W_{I}$ such that $E^{\prime}=\left[\Gamma^{\prime}\right]$. Let $d \in \Gamma^{\prime} \cap \mathcal{D}$. By 21.4 we have $\gamma_{d}^{W_{I}}=\left[\Gamma^{\prime}\right]=E^{\prime}$. By 20.17 we have $E=\mathbf{j}_{W_{I}}^{W}\left(E^{\prime}\right)=\mathbf{j}_{W_{I}}^{W}\left(\gamma_{d}^{W_{I}}\right)=\gamma_{d}^{W}$. Let $\Gamma$ be the left cell of $W$ that contains $d$. By 21.4 we have $\gamma_{d}^{W}=[\Gamma]$. Hence $E=[\Gamma]$.

Case 2. $E=\mathbf{j}_{W_{I}}^{W}\left(E^{\prime}\right) \otimes \operatorname{sgn}$ where $I \subset S, I \neq S$ and $E^{\prime} \in \operatorname{Con}\left(W_{I}\right)$. Then by Case $1, E \otimes \operatorname{sgn}=[\Gamma]$ for some left cell $\Gamma$ of $W$. By 21.5 we have $E=\left[\Gamma w_{0}\right]$. The lemma is proved.

Proposition 22.3. For any $E \in \operatorname{Irr} W$ there exists a constructible representation of $W$ which contains a simple component isomorphic to $E$.

The general case can be easily reduced to the case where $W$ is irreducible. Assume now that $W$ is irreducible. If $L=a l$ for some $a>0$, the constructible representations of $W$ are listed in [L7] and the proposition is easily checked. (See also the discussion of types $A, D$ in $22.5,22.26$.) In the cases where $W$ is irreducible but $L$ is not of the form al, the constructible representations are described later in this section and this yields the proposition in all cases.

22.4. Let $W=\mathfrak{S}_{n}$ be the group of permutations of $1,2, \ldots, n$. We regard $W$ as a Coxeter group with generators

$$
s_{1}=(1,2), s_{2}=(2,3), \ldots, s_{n-1}=(n-1, n),
$$

(transpositions). We take $L=a l$ where $a>0$.

The simple $W$-modules (up to isomorphism) are in 1-1 correspondence with the partitions $\alpha=\left(\alpha_{1} \geq \alpha_{2} \geq \ldots\right)$ such that $\alpha_{N}=0$ for large $N$ and $\sum_{i} \alpha_{i}=n$. The correspondence (denoted by $\alpha \mapsto \pi_{\alpha}$ ) is defined as follows. Let $\alpha$ be as above, let $\left(\alpha_{1}^{\prime} \geq \alpha_{2}^{\prime} \geq \ldots\right)$ be the partition dual to $\alpha$. Let $\pi_{\alpha}$ be the simple $W$-module whose 
restriction to $\mathfrak{S}_{\alpha_{1}} \times \mathfrak{S}_{\alpha_{2}} \ldots$ contains 1 and whose restriction to $\mathfrak{S}_{\alpha_{1}^{\prime}} \times \mathfrak{S}_{\alpha_{2}^{\prime}} \ldots$ contains the sign representation. We have (a consequence of results of Steinberg):

$$
f_{\left(\pi_{\alpha}\right)_{v}}=v^{-\sum_{i} 2\left(\begin{array}{c}
\alpha_{i}^{\prime} \\
2
\end{array}\right)}+\text { strictly higher powers of } v
$$

It follows that

(a) $\mathbf{a}_{\pi_{\alpha}}=\sum_{i} a\left(\begin{array}{c}\alpha_{i}^{\prime} \\ 2\end{array}\right)$ and $f_{\left(\pi_{\alpha}\right)_{\star}}=1$.

Lemma 22.5. In the setup of 22.4, a W-module is constructible if and only if it is simple.

For any sequence $\beta=\left(\beta_{1}, \beta_{2}, \ldots\right)$ in $\mathbf{N}$ such that $\beta_{N}=0$ for large $N$ and $\sum_{i} \beta_{i}=n$, we set

$$
I_{\beta}=\left\{s_{i} ; i \in[1, n-1], i \neq \beta_{1}, i \neq \beta_{1}+\beta_{2}, \ldots\right\}
$$

From 22.4(a) we see easily that, if $\beta$ is the same as $\alpha^{\prime}$ up to order, then

(a) $\mathbf{j}_{W_{I_{\beta}}}^{W}(\operatorname{sgn})=\pi_{\alpha}$.

Since the $\operatorname{sgn} \in \operatorname{Con}\left(W_{I_{\beta}}\right)$, it follows that $\pi_{\alpha} \in \operatorname{Con}(W)$. Thus any simple $W$ module is constructible.

We now show that any constructible representation $E$ of $W=\mathfrak{S}_{n}$ is simple. We may assume that $n \geq 1$ and that the analogous result is true for any $W_{I^{\prime}} \neq W$. We may assume that $E=\mathbf{j}_{W_{I_{\beta}}}^{W}(C)$ where $\beta$ is as above, $W_{I_{\beta}} \neq W$ and $C \in \operatorname{Con}\left(W_{I_{\beta}}\right)$. By the induction hypothesis, $C$ is simple. Since the analogue of (a) holds for $W_{I_{\beta}}$ (instead of $W$ ) we have $C=\mathbf{j}_{W_{I^{\prime}}}^{W_{I_{\beta}}}$ (sgn) for some $\beta^{\prime}$ such that $W_{I_{\beta^{\prime}}} \subset W_{I_{\beta}}$. By the transitivity of truncated induction we have $E=\mathbf{j}_{W_{I_{\beta^{\prime}}}}^{W}(\operatorname{sgn})$. Hence, by (a), for $\beta^{\prime}$ instead of $\beta, E$ is simple. The lemma is proved.

22.6. We now develop some combinatorics which is useful for the verification of 22.3 for $W$ of classical type.

Let $a>0, b \geq 0$ be integers. We can write uniquely $b=a r+b^{\prime}$ where $r, b^{\prime} \in \mathbf{N}$ and $b^{\prime}<a$. Let $N \in \mathbf{N}$. Let $\mathcal{M}_{a, b}^{N}$ be the set of multisets $\tilde{Z}=\left\{\tilde{z}_{1} \leq \tilde{z}_{2} \leq \cdots \leq\right.$ $\left.\tilde{z}_{2 N+r}\right\}$ of integers $\geq 0$ such that

(a) if $b^{\prime}=0$, there are at least $N+r$ distinct entries in $\tilde{Z}$, no entry is repeated more than twice and all entries of $\tilde{Z}$ are divisible by $a$;

(b) if $b^{\prime}>0$, all inequalities in $\tilde{Z}$ are strict and $N$ entries of $\tilde{Z}$ are divisible by $a$ and $N+r$ entries of $\tilde{Z}$ are equal to $b^{\prime}$ modulo $a$.

The entries which appear in $\tilde{Z}$ exactly once are called the singles of $\tilde{Z}$; they form a set $Z$. The other entries of $\tilde{Z}$ are called the doubles of $\tilde{Z}$.

For example, the multiset $\tilde{Z}^{0}$ whose entries are (up to order)

$$
0, a, 2 a, \ldots,(N-1) a, b^{\prime}, a+b^{\prime}, 2 a+b^{\prime}, \ldots,(N+r-1) a+b^{\prime}
$$


belongs to $\mathcal{M}_{a, b}^{N}$. Clearly, the sum of entries of $\tilde{Z}$ minus the sum of entries of $\tilde{Z}^{0}$ is $\geq 0$ and divisible by $a$, hence it is equal to $a n$ for a well defined $n \in \mathbf{N}$ said to be the $\operatorname{rank}$ of $\tilde{Z}$. We have

$$
\sum_{k=1}^{2 N+r} \tilde{z}_{k}=a n+a N^{2}+N(b-a)+a\left(\begin{array}{l}
r \\
2
\end{array}\right)+b^{\prime} r .
$$

Note that $\tilde{Z}^{0}$ has rank 0 . Let $\mathcal{M}_{a, b ; n}^{N}$ be the set of multisets of rank $n$ in $\mathcal{M}_{a, b}^{N}$. We define an (injective) map $\mathcal{M}_{a, b}^{N} \rightarrow \mathcal{M}_{a, b}^{N+1}$ by

$$
\left\{\tilde{z}_{1} \leq \tilde{z}_{2} \leq \cdots \leq \tilde{z}_{2 N+r}\right\} \mapsto\left\{0, b^{\prime}, \tilde{z}_{1}+a \leq \tilde{z}_{2}+a \leq \cdots \leq \tilde{z}_{2 N+r}+a\right\}
$$

This restricts for any $n \in \mathbf{N}$ to an (injective) map

(c) $\mathcal{M}_{a, b ; n}^{N} \rightarrow \mathcal{M}_{a, b ; n}^{N+1}$.

It is easy to see that, for fixed $n, \sharp\left(\mathcal{M}_{a, b ; n}^{N}\right)$ is bounded as $N \rightarrow \infty$, hence the maps (c) are bijections for large $N$. Let $\mathcal{M}_{a, b ; n}$ be the inductive limit of $\mathcal{M}_{a, b ; n}^{N}$ as $N \rightarrow \infty$ (with respect to the maps (c)).

22.7. Let $\mathrm{Sy}_{a, b ; n}^{N}$ be the set consisting of all tableaux (or symbols)

$$
\begin{array}{r}
\lambda_{1}, \lambda_{2}, \ldots, \lambda_{N+r} \\
\mu_{1}, \mu_{2}, \ldots, \mu_{N}
\end{array}
$$

where $\lambda_{1}<\lambda_{2}<\cdots<\lambda_{N+r}$ are integers $\geq 0$, congruent to $b^{\prime}$ modulo $a$, $\mu_{1}, \mu_{2}, \ldots, \mu_{N}$ are integers $\geq 0$, divisible by $a$ and

$$
\sum_{i} \lambda_{i}+\sum_{j} \mu_{j}=a n+a N^{2}+N(b-a)+a\left(\begin{array}{l}
r \\
2
\end{array}\right)+b^{\prime} r .
$$

If we arrange the entries of $\Lambda$ in a single row, we obtain a multiset $\tilde{Z} \in \mathcal{M}_{a, b ; n}^{N}$. This defines a (surjective) map $\pi_{N}: \mathrm{Sy}_{a, b ; n}^{N} \rightarrow \mathcal{M}_{a, b ; n}^{N}$. We define an (injective) map

(b) $\mathrm{Sy}_{a, b ; n}^{N} \rightarrow \mathrm{Sy}_{a, b ; n}^{N+1}$

by associating to (a) the symbol

$$
\begin{aligned}
b^{\prime}, \lambda_{1}+a, \lambda_{2}+a, \ldots, \lambda_{N+r}+a \\
0, \mu_{1}+a, \mu_{2}+a, \ldots, \mu_{N}+a .
\end{aligned}
$$

This is compatible with the map $\mathcal{M}_{a, b}^{N} \rightarrow \mathcal{M}_{a, b}^{N+1}$ in $22.6\left(\right.$ via $\pi_{N}, \pi_{N+1}$ ).

Since for fixed $n, \sharp\left(\mathrm{Sy}_{a, b ; n}^{N}\right)$ is bounded as $N \rightarrow \infty$, the maps (b) are bijections for large $N$. Let $\mathrm{Sy}_{a, b ; n}$ be the inductive limit of $\mathrm{Sy}_{a, b ; n}^{N}$ as $N \rightarrow \infty$ (with respect to the maps (b)). 
22.8. Let $\tilde{Z}=\left\{\tilde{z}_{1} \leq \tilde{z}_{2} \leq \cdots \leq \tilde{z}_{2 N+r}\right\} \in \mathcal{M}_{a, b ; n}^{N}$. Let $t$ be an integer which is large enough so that the multiset

(a) $\left\{a t+b^{\prime}-\tilde{z}_{1}, a t+b^{\prime}-\tilde{z}_{2}, \ldots, a t+b^{\prime}-\tilde{z}_{2 N+r}\right\}$

is contained in the multiset

(b) $\left\{0, a, 2 a, \ldots, t a, b^{\prime}, a+b^{\prime}, 2 a+b^{\prime}, \ldots, t a+b^{\prime}\right\}$

and let $\overline{\tilde{Z}}$ be the complement of (a) in (b). Then $\overline{\tilde{Z}} \in \mathcal{M}_{a, b}^{t+1-N-r}$. The sum of entries of $\overline{\tilde{Z}}$ is

$$
\begin{aligned}
& \sum_{k \in[0, t]}\left(2 k a+b^{\prime}\right)-\left(a t+b^{\prime}\right)(2 N+r)+\sum_{h} \tilde{z}_{h} \\
& =a t(t+1)+(t+1) b^{\prime}-\left(a t+b^{\prime}\right)(2 N+r)+a n+a N^{2}+N(b-a)+a\left(\begin{array}{l}
r \\
2
\end{array}\right)+b^{\prime} r \\
& =a n+a(t+1-N-r)^{2}+(t+1-N-r)(b-a)+a\left(\begin{array}{l}
r \\
2
\end{array}\right)+b^{\prime} r .
\end{aligned}
$$

Thus, $\overline{\tilde{Z}}$ has rank $n$.

We define a bijection $\pi_{N}^{-1}(\tilde{Z}) \stackrel{\sim}{\longrightarrow} \pi_{t+1-N-r}^{-1}(\overline{\tilde{Z}})$ by $\Lambda \mapsto \bar{\Lambda}$ where $\Lambda$ is as in $22.7(\mathrm{a})$ and $\bar{\Lambda}$ is

$$
\begin{aligned}
& \left\{b^{\prime}, a+b^{\prime}, 2 a+b^{\prime}, 3 a+b^{\prime}, \ldots, t a+b^{\prime}\right\}-\left\{a t+b^{\prime}-\mu_{1}, a t+b^{\prime}-\mu_{2}, \ldots, a t+b^{\prime}-\mu_{N}\right\} \\
& \{0, a, 2 a, 3 a, \ldots, t a\}-\left\{a t+b^{\prime}-\lambda_{1}, a t+b^{\prime}-\lambda_{2}, \ldots, a t+b^{\prime}-\lambda_{N+r}\right\}
\end{aligned}
$$

22.9. Let $W=W_{n}$ be the group of permutations of $1,2, \ldots, n, n^{\prime}, \ldots, 2^{\prime}, 1^{\prime}$ which commute with the involution $i \mapsto i^{\prime}, i^{\prime} \mapsto i$. We regard $W_{n}$ as a Coxeter group with generators $s_{1}, s_{2}, \ldots, s_{n}$ given as products of transpositions by

$$
\begin{aligned}
& s_{1}=(1,2)\left(1^{\prime}, 2^{\prime}\right), s_{2}=(2,3)\left(2^{\prime}, 3^{\prime}\right), \ldots, s_{n-1}=(n-1, n)\left((n-1)^{\prime}, n^{\prime}\right), \\
& s_{n}=\left(n, n^{\prime}\right) .
\end{aligned}
$$

22.10. A permutation in $W$ defines a permutation of the $n$ element set consisting of the pairs $\left(1,1^{\prime}\right),\left(2,2^{\prime}\right), \ldots,\left(n, n^{\prime}\right)$. Thus we have a natural homomorphism of $W_{n}$ onto $\mathfrak{S}_{n}$, the symmetric group in $n$ letters. Define a homomorphism $\chi_{n}$ : $W_{n} \rightarrow \pm 1$ by

$\chi_{n}(\sigma)=1$ if $\{\sigma(1), \sigma(2), \ldots, \sigma(n)\} \cap\left\{1^{\prime}, 2^{\prime}, \ldots, n^{\prime}\right\}$ has even cardinal, $\chi_{n}(\sigma)=-1$, otherwise.

The simple $W$-modules (up to isomorphism) are in 1-1 correspondence with the ordered pairs $\alpha, \beta$ where $\alpha=\left(\alpha_{1} \geq \alpha_{2} \geq \ldots\right)$ and $\beta=\left(\beta_{1} \geq \beta_{2} \geq \ldots\right)$ are partitions such that $\alpha_{N}=\beta_{N}=0$ for large $N$ and $\sum_{i} \alpha_{i}+\sum_{j} \beta_{j}=n$. The correspondence (denoted by $\alpha, \beta \mapsto E^{\alpha, \beta}$ ) is defined as follows. Let $\alpha, \beta$ be as above, let $\left(\alpha_{1}^{\prime} \geq \alpha_{2}^{\prime} \geq \ldots\right)$ be the partition dual to $\alpha$ and let $\left(\beta_{1}^{\prime} \geq \beta_{2}^{\prime} \geq \ldots\right)$ be the partition dual to $\beta$. Let $k=\sum_{i} \alpha_{i}, l=\sum_{j} \beta_{j}$. Let $\pi_{\alpha}$ be the simple $\mathfrak{S}_{k^{-}}$ module defined as in 22.4 and let $\pi_{\beta}$ be the analogously defined simple $\mathfrak{S}_{l}$-module. We regard $\pi_{\alpha}, \pi_{\beta}$ as simple modules of $W_{k}, W_{l}$ via the natural homomorphisms 
$W_{k} \rightarrow \mathfrak{S}_{k}, W_{l} \rightarrow \mathfrak{S}_{l}$ as above. We identify $W_{k} \times W_{l}$ with the subgroup of $W$ consisting of all permutations in $W$ which map $1,2, \ldots, k, k^{\prime}, \ldots, 2^{\prime}, 1^{\prime}$ into itself hence also map $k+1, k+2, \ldots, n, n^{\prime}, \ldots,(k+2)^{\prime},(k+1)^{\prime}$ into itself. Consider the representation $\pi_{\alpha} \otimes\left(\pi_{\beta} \otimes \chi_{l}\right)$ of $W_{k} \times W_{l}$. We induce it to $W$; the resulting representation of $W$ is irreducible; we denote it by $E^{\alpha, \beta}$.

We fix $a>0, b \geq 0$ and we write $b=a r+b^{\prime}$ as in 22.6.

Let $\alpha, \beta$ be as in 22.10. Let $N$ be an integer such that $\alpha_{N+r+1}=0, \beta_{N+1}=0$. (Any large enough integer satisfies these conditions.) We set

$\lambda_{i}=a\left(\alpha_{N+r-i+1}+i-1\right)+b^{\prime},(i \in[1, N+r]), \quad \mu_{j}=a\left(\beta_{N-j+1}+j-1\right),(j \in[1, N])$.

We have $0 \leq \lambda_{1}<\lambda_{2}<\cdots<\lambda_{N+r}, 0 \leq \mu_{1}<\mu_{2}<\cdots<\mu_{N}$. Let $\Lambda$ denote the tableau 22.7(a). It is easy to see that $\Lambda \in \mathrm{Sy}_{a, b ; n}^{N}$. Moreover, if $N$ is replaced by $N+1$, then $\Lambda$ is replaced by its image under $\operatorname{Sy}_{a, b ; n}^{N} \rightarrow \operatorname{Sy}_{a, b ; n}^{N+1}$ (see 22.7). Let $[\Lambda]=E^{\alpha, \beta}$. Note that $[\Lambda]$ depends only on the image of $\Lambda$ under the canonical map $\mathrm{Sy}_{a, b ; n}^{N} \rightarrow \mathrm{Sy}_{a, b ; n}$. In this way, we see that

the simple $W$-modules are naturally in bijection with the set $\mathrm{Sy}_{a, b ; n}$.

For $i \in[1, N]$ we have $a\left(\alpha_{N-i+1}+i-1\right)+b=a\left(\alpha_{N+r-i-r+1}+i+r-1\right)+b^{\prime}=\lambda_{i+r}$.

If $N$ is large we have $\lambda_{i}=a(i-1)+b^{\prime}$ for $i \in[1, r]$ and $\mu_{j}=a(j-1)$ for $j \in[1, r]$.

22.11. Let $q, y$ be indeterminates. With the notation in 22.15 , let

$$
\begin{gathered}
H_{\alpha}(q)=q^{-\sum_{i}\left(\begin{array}{c}
\alpha_{i}^{\prime} \\
2
\end{array}\right)} \prod_{i, j} \frac{q^{\alpha_{i}+\alpha_{j}^{\prime}-i-j+1}-1}{q-1}, \\
G_{\alpha, \beta}(q, y)=q^{-\sum_{i} \alpha_{i}^{\prime} \beta_{i}^{\prime} / 2} \prod_{i, j}\left(q^{\alpha_{i}+\beta_{j}^{\prime}-i-j+1} y+1\right) ;
\end{gathered}
$$

both products are taken over all $i \geq 1, j \geq 1$ such that $\alpha_{i} \geq j, \alpha_{j}^{\prime} \geq i$.

Define a weight function $L: W \rightarrow \mathbf{N}$ by $L\left(s_{1}\right)=L\left(s_{2}\right)=\cdots=L\left(s_{n-1}\right)=a$, $L\left(s_{n}\right)=b$. We now assume that both $a, b$ are $>0$. We also assume that $a, b$ are such that $W, L$ satisfies the assumptions of 18.1. Then $f_{E_{v}^{\alpha, \beta}}$ is defined in terms of this $L$.

Lemma 22.12 (Hoefsmit $[\mathrm{H}]$ ). We have

$$
f_{E_{v}^{\alpha, \beta}}=H_{\alpha}\left(v^{2 a}\right) H_{\beta}\left(v^{2 a}\right) G_{\alpha, \beta}\left(v^{2 a}, v^{2 b}\right) G_{\beta, \alpha}\left(v^{2 a}, v^{-2 b}\right) .
$$

We will rewrite the expression above using the following result.

Lemma 22.13. Let $N$ be a large integer. We have

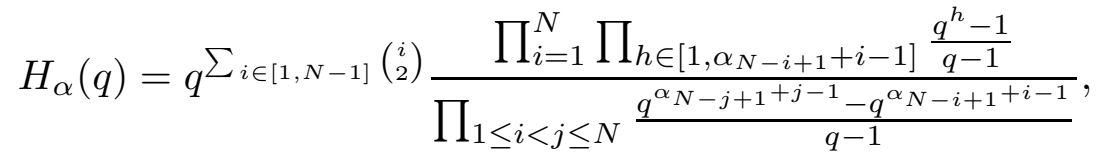




$$
\begin{aligned}
& G_{\alpha, \beta}(q, y) G_{\beta, \alpha}\left(q, y^{-1}\right)=q^{\sum_{i \in[1, N-1]} i^{2}}\left(\sqrt{y}+\sqrt{y}^{-1}\right)^{N} \\
& \times \frac{\prod_{i=1}^{N} \prod_{h \in\left[1, \alpha_{N-i+1}+i-1\right]}\left(q^{h} y+1\right) \prod_{j=1}^{N} \prod_{h \in\left[1, \beta_{N-j+1}+j-1\right]}\left(q^{h} y^{-1}+1\right)}{\prod_{i, j \in[1, N]}\left(q^{\alpha_{N-i+1}+i-1} \sqrt{y}+q^{\beta_{N-j+1}+j-1} \sqrt{y}^{-1}\right)} .
\end{aligned}
$$

The proof is by induction on $n$. We omit it.

Proposition 22.14. (a) If $b^{\prime}=0$ then $f_{[\Lambda]}$ is equal to $2^{d}$ where $2 d+r$ is the number of singles in $\Lambda$. If $b^{\prime}>0$ then $f_{[\Lambda]_{\bullet}}=1$.

(b) We have $\mathbf{a}_{[\Lambda]}=A_{N}-B_{N}$ where

$$
\begin{aligned}
A_{N} & =\sum_{i \in[1, N+r], j \in[1, N]} \min \left(\lambda_{i}, \mu_{j}\right)+\sum_{1 \leq i<j \leq N+r} \min \left(\lambda_{i}, \lambda_{j}\right)+\sum_{1 \leq i<j \leq N} \min \left(\mu_{i}, \mu_{j}\right), \\
B_{N} & =\sum_{i \in[1, N+r], j \in[1, N]} \min \left(a(i-1)+b^{\prime}, a(j-1)\right) \\
& +\sum_{1 \leq i<j \leq N+r} \min \left(a(i-1)+b^{\prime}, a(j-1)+b^{\prime}\right)+\sum_{1 \leq i<j \leq N} \min (a(i-1), a(j-1)) .
\end{aligned}
$$

It is enough to prove (a) assuming that $N$ is large. Since

$$
A_{N+1}-A_{N}=a(N+r) N+a\left(\begin{array}{c}
N \\
2
\end{array}\right)+a\left(\begin{array}{c}
N+r \\
2
\end{array}\right)+b^{\prime} N+b^{\prime}(N+r)=B_{N+1}-B_{N},
$$

we have $A_{N+1}-B_{N+1}=A_{N}-B_{N}$ hence it is enough to prove (b) assuming that $N$ is large. In the remainder of the proof we assume that $N$ is large.

For $f, f^{\prime} \in \mathbf{C}(v)$ we write $f \cong f^{\prime}$ if $f^{\prime}=f g$ with $g \in \mathbf{C}(v),\left.g\right|_{v=0}=1$. Using $22.12,22.13$, we see that

$$
\begin{gathered}
f_{[\Lambda]_{v}} \cong \prod_{i \in[1, N]}\left(v^{2 a-2 b}+1\right)\left(v^{4 a-2 b}+1\right) \ldots\left(v^{2 \mu_{i}-2 b}+1\right) \\
\left(v^{b}+v^{-b}\right)^{N} v^{2 a \sum_{i=1}^{N-1}\left(2 i^{2}-i\right)} \prod_{i, j \in[1, N]}\left(v^{2 \lambda_{i+r}-b}+v^{2 \mu_{j}-b}\right)^{-1} \\
\prod_{1 \leq i<j \leq N}\left(v^{2 \lambda_{j+r}-2 b}-v^{2 \lambda_{i+r}-2 b}\right)^{-1} \prod_{1 \leq i<j \leq N}\left(v^{2 \mu_{j}}-v^{2 \mu_{i}}\right)^{-1}
\end{gathered}
$$

hence

$$
f_{[\Lambda]_{v}}=2^{d} v^{-K}+\text { strictly higher powers of } v
$$

where $d=0$ if $b^{\prime}>0$,

$$
\begin{aligned}
& d=\sharp\left(j \in[1, N]: b \leq \mu_{j}\right)-\sharp\left(i, j \in[1, N]: \lambda_{i+r}=\mu_{j}\right) \\
& =N-\sharp\left(i \in[1, r], j \in[1, N]:(i-1) a=\mu_{j}\right)-\sharp\left(i, j \in[1, N]: \lambda_{i+r}=\mu_{j}\right) \\
& =N-\sharp\left(i \in[1, r], j \in[1, N]: \lambda_{i}=\mu_{j}\right)-\sharp\left(i, j \in[1, N]: \lambda_{i+r}=\mu_{j}\right) \\
& =N-\sharp\left(i \in[1, N+r], j \in[1, N]: \lambda_{i}=\mu_{j}\right)=(\sharp \text { singles }-r) / 2,
\end{aligned}
$$


if $b^{\prime}=0$,

$$
\begin{aligned}
& -K=-b N+2 a \sum_{i \in[1, N-1]}\left(2 i^{2}-i\right)+\sum_{j \in[1, N]} \sum_{\substack{k \in[1, r] \\
a k \leq \mu_{j}}}(2 a k-2 b) \\
& -\sum_{i, j \in[1, N]}\left(-b+2 \min \left(\lambda_{i+r}, \mu_{j}\right)\right)-\sum_{1 \leq i<j \leq N}\left(-2 b+2 \min \left(\lambda_{i+r}, \lambda_{j+r}\right)\right) \\
& -\sum_{1 \leq i<j \leq N} 2 \min \left(\mu_{i}, \mu_{j}\right)=-b N+2 a \sum_{i \in[1, N-1]}\left(2 i^{2}-i\right)+2 b N^{2}-b N \\
& +\sum_{j \in[1, N]} \sum_{k \in[1, r], a k \leq \mu_{j}}(2 a k-2 b)-\sum_{i, j \in[1, N]} 2 \min \left(\lambda_{i+r}, \mu_{j}\right) \\
& -\sum_{1 \leq i<j \leq N} 2 \min \left(\lambda_{i+r}, \lambda_{j+r}\right)-\sum_{1 \leq i<j \leq N} 2 \min \left(\mu_{i}, \mu_{j}\right) \\
& =\sum_{j \in[1, N]} \sum_{k \in[1, r], a k \leq \mu_{j}}(2 a k-2 b)-\sum_{i, j \in[1, N]} 2 \min \left(\lambda_{i+r}, \mu_{j}\right) \\
& -\sum_{1 \leq i<j \leq N} 2 \min \left(\lambda_{i+r}, \lambda_{j+r}\right)-\sum_{1 \leq i<j \leq N} 2 \min \left(\mu_{i}, \mu_{j}\right)+{ }^{\star} .
\end{aligned}
$$

(We will generally write ${ }^{\star}$ for an expression which depends only on $a, b, N$.) We have

$$
\begin{aligned}
& \sum_{\substack{j \in[1, N] \\
k \in[1, r] \\
a k \leq \mu_{j}}}(2 a k-2 b)=\sum_{\substack{j \in[1, r] \\
k \in[1, r] \\
a k \leq \mu_{j}}}(2 a k-2 b)+\sum_{\substack{j \in[r+1, N] \\
k \in[1, r] \\
a k \leq \mu_{j}}}(2 a k-2 b) \\
& =\sum_{\substack{j \in[1, r] \\
k \in[1, r] \\
a k \leq a(j-1)}}(2 a k-2 b)+\sum_{\substack{j \in[r+1, N] \\
k \in[1, r]}}(2 a k-2 b)=\star \\
& k
\end{aligned}
$$

hence

$$
\begin{aligned}
& -K \\
& =-2\left(\sum_{i, j \in[1, N]} \min \left(\lambda_{i+r}, \mu_{j}\right)-\sum_{1 \leq i<j \leq N} \min \left(\lambda_{i+r}, \lambda_{j+r}\right)-\sum_{1 \leq i<j \leq N} \min \left(\mu_{i}, \mu_{j}\right)\right) \\
& +\star \text {. }
\end{aligned}
$$

We have

$$
\begin{aligned}
& \sum_{i \in[1, r], j \in[1, N]} \min \left(\lambda_{i}, \mu_{j}\right)=\sum_{i \in[1, r], j \in[1, N]} \min \left(a(i-1)+b^{\prime}, \mu_{j}\right) \\
= & \sum_{i \in[1, r], j \in[1, r]} \min \left(a(i-1)+b^{\prime}, a(j-1)\right)+\sum_{i \in[1, r], j \in[r+1, N]} \min \left(a(i-1)+b^{\prime}, \mu_{j}\right) \\
= & \sum_{i \in[1, r], j \in[1, r]} \min \left(a(i-1)+b^{\prime}, a(j-1)\right)+\sum_{i \in[1, r], j \in[r+1, N]}\left(a(i-1)+b^{\prime}\right)=\star
\end{aligned}
$$


hence

$$
\sum_{i, j \in[1, N]} \min \left(\lambda_{i+r}, \mu_{j}\right)=\sum_{i \in[1, N+r], j \in[1, N]} \min \left(\lambda_{i}, \mu_{j}\right)+{ }^{\star}
$$

We have

$$
\begin{aligned}
& \sum_{1 \leq i<j \leq N+r} \min \left(\lambda_{i}, \lambda_{j}\right)=\sum_{1 \leq i<j \leq N} \min \left(\lambda_{i+r}, \lambda_{j+r}\right)+\sum_{i \in[1, r]} \lambda_{i}(N+r-i) \\
= & \sum_{1 \leq i<j \leq N} \min \left(\lambda_{i+r}, \lambda_{j+r}\right)+\sum_{i \in[1, r]}\left(a(i-1)+b^{\prime}\right)(N+r-i) \\
= & \sum_{1 \leq i<j \leq N} \min \left(\lambda_{i+r}, \lambda_{j+r}\right)+\star
\end{aligned}
$$

We see that

$$
-K=-2 A_{N}+\star
$$

In the special case where $\alpha=\beta=(0 \geq 0 \geq \ldots)$ we have $K=0$. On the other hand, by (c), we have $0=-2 B_{N}+{ }^{\star}$ where ${ }^{\star}$ is as in (c). Hence in general we have $-K=-2 A_{N}+2 B_{N}$. This proves the proposition, in view of 20.11 and $20.21(\mathrm{a})$.

22.15. We identify $\mathfrak{S}_{k} \times W_{l}(k+l=n)$ with the subgroup of $W$ consisting of all permutations in $W$ which map $\{1,2, \ldots, k\}$ into itself (hence also map $\left\{1,2, \ldots, k^{\prime}\right\}$ and $\left\{k+1, \ldots, n, n^{\prime} \ldots,(k+1)^{\prime}\right\}$ into themselves. This is a standard parabolic subgroup of $W$. We consider an irreducible representation of $\mathfrak{S}_{k} \times W_{l}$ of the form $\operatorname{sgn}_{k} \otimes\left[\Lambda^{\prime}\right]$ where $\operatorname{sgn}_{k}$ is the sign representation of $\mathfrak{S}_{k}$ and $\Lambda^{\prime} \in \operatorname{Sy}_{a, b ; l}^{N}$. We may assume that $\Lambda^{\prime}$ has at least $k$ entries. We want to associate to $\Lambda^{\prime}$ a symbol in $\mathrm{Sy}_{a, b ; n}^{N}$ by increasing each of the $k$ largest entries in $\Lambda^{\prime}$ by $a$. It may happen that the set of $r$ largest entries of $\Lambda^{\prime}$ is not uniquely defined but there are two choices for it. (This can only happen if $b^{\prime}=0$.) Then the same procedure gives rise to two distinct symbols $\Lambda^{I}, \Lambda^{I I}$ in $\mathrm{Sy}_{a, b ; n}^{N}$.

Lemma 22.16. (a) $g_{\left(\operatorname{sgn}_{k} \otimes\left[\Lambda^{\prime}\right]\right)_{\star}}=g_{\left[\Lambda^{\prime}\right]_{\star}}$ is equal to $g_{[\Lambda]_{\star}}$ or to $g_{\left[\Lambda^{I}\right]_{\star}}+g_{\left[\Lambda^{I I}\right]_{\star}}$.

(b) $\mathbf{a}_{\mathrm{sgn}_{k} \otimes\left[\Lambda^{\prime}\right]}=a\left(\begin{array}{c}k \\ 2\end{array}\right)+\mathbf{a}_{\left[\Lambda^{\prime}\right]}$ is equal to $\mathbf{a}_{[\Lambda]}$ or to $\mathbf{a}_{\left[\Lambda^{I}\right]}=\mathbf{a}_{\left[\Lambda^{I I}\right]}$.

$\Lambda$, if defined, has the same number of singles as $\Lambda^{\prime}$. Moreover, $\Lambda^{I}$ (and $\Lambda^{I I}$ ), if defined, has one more single than $\Lambda^{\prime}$. Hence (a) follows from 22.14(a) using 20.18, 20.19 .

By 22.14(b), the difference $\mathbf{a}_{[\tilde{\Lambda}]}-\mathbf{a}_{[\Lambda]}$ (where $\tilde{\Lambda}$ is either $\Lambda$ or $\Lambda^{I}$ or $\Lambda^{I I}$ ) is $a$ times the number of $i<j$ in $[1, k]$. Thus, it is $a\left(\begin{array}{l}k \\ 2\end{array}\right)$. Hence (a) follows from 20.18, 20.19. The lemma is proved.

Lemma 22.17. $\mathbf{j}_{\mathfrak{S}_{k} \times W_{l}}^{W}\left(\operatorname{sgn}_{k} \otimes\left[\Lambda^{\prime}\right]\right)$ equals $[\Lambda]$ or $\left[\Lambda^{I}\right]+\left[\Lambda^{I I}\right]$.

By a direct computation (involving representations of symmetric groups) we see that: 
(a) if $\Lambda$ is defined then $\left[\left[\Lambda^{\prime}\right]:[\Lambda]\right] \geq 1$;

(b) if $\Lambda^{I}, \Lambda^{I I}$ are defined then $\left[\left[\Lambda^{\prime}\right]:\left[\Lambda^{I}\right]\right] \geq 1$ and $\left[\left[\Lambda^{\prime}\right]:\left[\Lambda^{I I}\right]\right] \geq 1$.

In the setup of (a) we have (by 20.14(b)):

$g_{\left[\Lambda^{\prime}\right]_{\boldsymbol{\star}}}=\sum_{E ; \mathbf{a}_{E}=\mathbf{a}_{E^{\prime}}}\left[\left[\Lambda^{\prime}\right]: E\right] g_{E_{\boldsymbol{\star}}}$ hence using $22.16($ a) we have

$$
g_{[\Lambda]_{\boldsymbol{\iota}}}=\sum_{E ; \mathbf{a}_{E}=\mathbf{a}_{E^{\prime}}}\left[\left[\Lambda^{\prime}\right]: E\right] g_{E_{\boldsymbol{\omega}}} .
$$

By 22.16(b), $E=[\Lambda]$ enters in the last sum and its contribution is $\geq g_{[\Lambda]}$; the contribution of the other $E$ is $\geq 0$ (see 20.13(b)). Hence (c) forces $\left[\left[\Lambda^{\prime}\right]:[\Lambda]\right]=1$ and $\left[\left[\Lambda^{\prime}\right]: E\right]=0$ for all other $E$ in the sum. In this case the lemma follows.

In the setup of (b) we have (by 20.14(b)) $g_{\left[\Lambda^{\prime}\right]_{\boldsymbol{\bullet}}}=\sum_{E ; \mathbf{a}_{E}=\mathbf{a}_{E^{\prime}}}\left[\left[\Lambda^{\prime}\right]: E\right] g_{E_{\boldsymbol{\omega}}}$ hence, by $22.16(\mathrm{a})$,

$$
g_{\left[\Lambda^{I}\right]_{\boldsymbol{\iota}}}+g_{\left[\Lambda^{I I}\right]_{\bullet}}=\sum_{E ; \mathbf{a}_{E}=\mathbf{a}_{E^{\prime}}}\left[\left[\Lambda^{\prime}\right]: E\right] g_{E_{\boldsymbol{\star}}}
$$

By 22.16(b), $E=\left[\Lambda^{I}\right]$ and $E=\left[\Lambda^{I I}\right]$ enter in the last sum and their contribution is $\geq g_{\left[\Lambda^{I}\right]_{\star}}+g_{\left[\Lambda^{I I}\right]_{\star}}$; the contribution of the other $E$ is $\geq 0$ (see 20.13(b)). Hence (d) forces $\left[\left[\Lambda^{\prime}\right]:\left[\Lambda^{I}\right]\right]=\left[\left[\Lambda^{\prime}\right]:\left[\Lambda^{I I}\right]\right]=1$ and $\left[\left[\Lambda^{\prime}\right]: E\right]=0$ for all other $E$ in the sum. The lemma follows.

Lemma 22.18. $[\Lambda] \otimes \operatorname{sgn}=[\bar{\Lambda}]$. (Notation of 22.14.)

This can be reduced to a known statement about the symmetric group. We omit the details.

22.19. Let $Z$ be a totally ordered finite set $z_{1}<z_{2}<\cdots<z_{M}$. For any $r \in[0, M]$ such that $r=M \bmod 2$ let $\underline{Z}_{r}$ be the set of subsets of $Z$ of cardinal $(M-r) / 2$. An involution $\iota: Z \rightarrow Z$ is said to be $r$-admissible if the following hold:

(a) $\iota$ has exactly $r$ fixed points;

(b) if $M=r$, there is no further condition; if $M>r$, there exist two consecutive elements $z, z^{\prime}$ of $Z$ such that $\iota(z)=z^{\prime}, \iota\left(z^{\prime}\right)=z$ and the induced involution of $Z-\left\{z, z^{\prime}\right\}$ is $r$-admissible.

Let $\operatorname{Inv}_{r}(Z)$ be the set of $r$-admissible involutions of $Z$. To $\iota \in \operatorname{Inv}_{r}(Z)$ we associate a subset $\mathcal{S}_{\iota}$ of $\underline{Z}_{r}$ as follows: a subset $Y \subset Z$ is in $\mathcal{S}_{\iota}$ if it contains exactly one element in each non-trivial $\iota$-orbit. Clearly, $\sharp\left(\mathcal{S}_{\iota}\right)=2^{p_{0}}$ where $p_{0}=(M-r) / 2$. (In fact, $\mathcal{S}_{\iota}$ is naturally an affine space over the field $\mathbf{F}_{2}$.)

Lemma 22.20. Assume that $p_{0}>0$. Let $Y \in \underline{Z}_{r}$.

(a) We can find two consecutive elements $z, z^{\prime}$ of $Z$ such that exactly one of $z, z^{\prime}$ is in $Y$.

(b) There exists $\iota \in \operatorname{Inv}_{r}(Z)$ such that $Y \in \mathcal{S}_{\iota}$. 
(c) Assume that for some $k \in\left[0, p_{0}-1\right], z_{1}, z_{2}, \ldots, z_{k}$ belong to $Y$ but $z_{k+1} \notin Y$. Let $l$ be the smallest number such that $l>k$ and $z_{l} \in Y$. There exists $\iota \in \operatorname{Inv}_{r}(Z)$ such that $Y \in \mathcal{S}_{\iota}$ and $\iota\left(z_{l}\right)=z_{l-1}$.

We prove (a). Let $z_{k}$ be the smallest element of $Y$. If $k>1$ then we can take $\left(z, z^{\prime}\right)=\left(z_{k-1}, z_{k}\right)$. Hence we may assume that $z_{1} \in Y$. Let $z_{k^{\prime}}$ be the next smallest element of $Y$. If $k^{\prime}>2$ then we can take $\left(z, z^{\prime}\right)=\left(z_{k^{\prime}-1}, z_{k^{\prime}}\right)$. Continuing like this we see that we may assume that $Y=\left\{z_{1}, z_{2}, \ldots, z_{p_{0}}\right\}$. Since $p_{0}<M$, we may take $\left(z, z^{\prime}\right)=\left(z_{p_{0}}, z_{p_{0}+1}\right)$.

We prove (b). Let $z, z^{\prime}$ be as in (a). Let $Z^{\prime}=Z-\left\{z, z^{\prime}\right\}$ with the induced order. Let $Y^{\prime}=Y \cap Z^{\prime}$. If $p_{0} \geq 2$ then by induction on $p_{0}$ we may assume that there exists $\iota^{\prime} \in \operatorname{Inv}_{r}\left(Z^{\prime}\right)$ such that $Y^{\prime} \in \mathcal{S}_{\iota^{\prime}}$. Extend $\iota^{\prime}$ to an involution $\iota$ of $Z$ by $z \mapsto z^{\prime}, z^{\prime} \mapsto z$. Then $\iota \in \operatorname{Inv}_{r}(Z)$ and $Y \in \mathcal{S}_{\iota}$. If $p_{0}=1$, define $\iota: Z \rightarrow Z$ so that $z \mapsto z^{\prime}, z^{\prime} \mapsto z$ and $\iota=1$ on $Z-\left\{z, z^{\prime}\right\}$. Then $\iota \in \operatorname{Inv}_{r}(Z)$ and $Y \in \mathcal{S}_{\iota}$.

We prove $(\mathrm{c})$. We have $l \geq k+2$. Hence $z_{l-1} \notin Y$. Let $\left(z, z^{\prime}\right)=\left(z_{l-1}, z_{l}\right)$. We continue as in the proof of (b), except that instead of invoking an induction hypothesis, we invoke (b) itself.

22.21. Assume that $M>r$. We consider the graph whose set of vertices is $\underline{Z}_{r}$ and in which two vertices $Y \neq Y^{\prime}$ are joined if there exists $\iota \in \operatorname{Inv}_{r}(Z)$ such that $Y \in \mathcal{S}_{\iota}, Y^{\prime} \in \mathcal{S}_{\iota}$.

Lemma 22.22. This graph is connected.

We show that any vertex $Y=\left\{z_{i_{1}}, z_{i_{2}}, \ldots, z_{i_{p_{0}}}\right\}$ is in the same connected component as $Y_{0}=\left\{z_{1}, z_{2}, \ldots, z_{p_{0}}\right\}$. We argue by induction on $m_{Y}=i_{1}+i_{2}+$ $\cdots+i_{p_{0}}$. If $m_{Y}=1+2+\cdots+p_{0}$ then $Y=Y_{0}$ and there is nothing to prove. Assume now that $m>1+2+\cdots+p_{0}$ so that $Y \neq Y_{0}$. Then the assumption of Lemma 22.20(c) is satisfied. Hence we can find $l$ such that $z_{l} \in Y, z_{l-1} \notin Y$ and $\iota \in \operatorname{Inv}_{r}(Z)$ such that $Y \in \mathcal{S}_{\iota}$ and $\iota\left(z_{l}\right)=z_{l-1}$. Let $Y^{\prime}=\left(Y-\left\{z_{l}\right\}\right) \cup\left\{z_{l-1}\right\}$. Then $Y^{\prime} \in \mathcal{S}_{\iota}$ hence $Y, Y^{\prime}$ are joined in our graph. We have $m_{Y^{\prime}}=m_{Y}-1$ hence by the induction hypothesis $Y^{\prime}, Y_{0}$ are in the same connected component. It follows that $Y, Y_{0}$ are in the same connected component. The lemma is proved.

22.23. Assume that $b^{\prime}=0$. Let $\tilde{Z} \in \mathcal{M}_{a, b ; n}^{N}$. Let $Z$ be the set of singles of $\tilde{Z}$. Each set $Y \in \underline{Z}_{r}$ gives rise to a symbol $\Lambda_{Y}$ in $\pi_{N}^{-1}(\tilde{Z})$ : the first row of $\Lambda_{Y}$ consists of $Z-Y$ and one element in each double of $\tilde{Z}$; the second row consists of $Y$ and one element in each double of $\tilde{Z}$. For any $\iota \in \operatorname{Inv}_{r}(Z)$ we set

$$
c(\tilde{Z}, \iota)=\oplus_{Y \in \mathcal{S}_{\iota}}\left[\Lambda_{Y}\right] \in \operatorname{Mod} W .
$$

Proposition 22.24. (a) In the setup of 22.23, let $\iota \in \operatorname{Inv}_{r}(Z)$. Then $c(\tilde{Z}, \iota) \in$ $\operatorname{Con}(W)$.

(b) All constructible representations of $W$ are obtained as in (a).

We prove (a) by induction on $n$. If $n=0$ the result is clear. Assume that $n \geq 1$. We may assume that 0 is not a double of $\tilde{Z}$. Let at be the largest entry of $\tilde{Z}$. 
(A) Assume that there exists $i, 0 \leq i<t$, such that ai does not appear in $\tilde{Z}$. Then $\tilde{Z}$ is obtained from $\tilde{Z}^{\prime} \in \mathcal{M}_{a, b ; n-k}^{N}$ with $n-k<n$ by increasing each of the $k$ largest entries by $a$ and this set of largest entries is unambiguously defined. The set $Z^{\prime}$ of singles of $\tilde{Z}^{\prime}$ is naturally in order preserving bijection with $Z$. Let $\iota^{\prime}$ correspond to $\iota$ under this bijection. By the induction hypothesis, $c\left(\tilde{Z}^{\prime}, \iota^{\prime}\right) \in$ $\operatorname{Con}\left(W_{n-k}\right)$. Since, by 22.5 , the sign representation $\operatorname{sgn}_{k}$ of $\mathfrak{S}_{k}$ is constructible, it follows that $\operatorname{sgn}_{k} \otimes c\left(\tilde{Z}^{\prime}, \iota^{\prime}\right) \in \operatorname{Con}\left(\mathfrak{S}_{k} \times W_{n-k}\right)$. Using 22.17 , we have

$$
\mathbf{j}_{\mathfrak{S}_{k} \times W_{n-k}}^{W}\left(\operatorname{sgn}_{k} \otimes c\left(\tilde{Z}^{\prime}, \iota^{\prime}\right)\right)=c(\tilde{Z}, \iota)
$$

hence $c(\tilde{Z}, \iota) \in \operatorname{Con}(W)$.

(B) Assume that there exists $i, 0<i \leq t$ such that ai is a double of $\tilde{Z}$. Let $\bar{Z}$ be as in 22.8 (with respect to our $t$ ). Then 0 is not a double of $\overline{\tilde{Z}}$ and the largest entry of $\bar{Z}$ is at. Let $\bar{Z}$ be the set of singles of $\bar{Z}$. We have $\bar{Z}=\{a t-z ; z \in Z\}$. Thus $\bar{Z}, Z$ are naturally in (order reversing) bijection under $j \mapsto a t-j$. Let $\iota^{\prime} \in \operatorname{Inv}_{r}(\bar{Z})$ correspond to $\iota$ under this bijection. Since at - ai does not appear in $\bar{Z},(\mathrm{~A})$ is applicable to $\tilde{Z}$. Hence $c\left(\bar{Z}, \iota^{\prime}\right) \in \operatorname{Con}(W)$. By 22.18 we have $c\left(\overline{\tilde{Z}}, \iota^{\prime}\right) \otimes \operatorname{sgn}=c(\tilde{Z}, \iota)$ hence $c(\tilde{Z}, \iota) \in \operatorname{Con}(W)$.

(C) Assume that we are not in case (A) and not in case (B). Then $\tilde{Z}=$ $\{0, a, 2 a, \ldots, t a\}=Z$. We can find $i a,(i+1) a$ in $Z$ such that $\iota$ interchanges $i a,(i+1) a$ and induces on $Z-\{i a,(i+1) a\}$ an $r$-admissible involution $\iota_{1}$. We have

$$
\tilde{Z}^{\prime}=\{0, a, 2 a, \ldots, i a, i a,(i+1) a,(i+2) a, \ldots,(t-1) a\} \in \mathcal{M}_{a, b ; n-k}^{N}
$$

with $n-k<n$. The set of singles of $\tilde{Z}^{\prime}$ is

$$
Z^{\prime}=\{0, a, 2 a, \ldots,(i-1) a,(i+1) a, \ldots,(t-1) a\} .
$$

It is in natural (order preserving) bijection with $Z-\{i a,(i+1) a\}$. Hence $\iota_{1}$ induces $\iota^{\prime} \in \operatorname{Inv}_{r}\left(Z^{\prime}\right)$. By the induction hypothesis we have $c\left(\tilde{Z}^{\prime}, \iota^{\prime}\right) \in \operatorname{Con}\left(W_{n-k}\right)$. Hence $\operatorname{sgn}_{k} \otimes c\left(\tilde{Z}^{\prime}, \iota^{\prime}\right) \in \operatorname{Con}\left(\mathfrak{S}_{k} \times W_{n-k}\right)$ where $\operatorname{sgn}_{k}$ is as in (A). By 22.17 we have

$$
\mathbf{j}_{\mathfrak{S}_{k} \times W_{n-k}}^{W}\left(\operatorname{sgn}_{k} \otimes c\left(\tilde{Z}^{\prime}, \iota^{\prime}\right)\right)=c(\tilde{Z}, \iota)
$$

hence $c(\tilde{Z}, \iota) \in \operatorname{Con}(W)$. This proves (a).

We prove (b) by induction on $n$. If $n=0$ the result is clear. Assume now that $n \geq 1$. By an argument like the ones used in (B) we see that the class of representations of $W$ obtained in (a) is closed under $\otimes \operatorname{sgn}$. Therefore, to show that $C \in \operatorname{Con}(W)$ is obtained in (a), we may assume that $C=\mathbf{j}_{\mathfrak{S}_{k} \times W_{n-k}}^{W}\left(C^{\prime}\right)$ for some $k>0$ and some $C^{\prime} \in \operatorname{Con}\left(\mathfrak{S}_{k} \times W_{n-k}\right)$. By 22.5 we have $C^{\prime}=E \otimes C^{\prime \prime}$ where $E$ is a simple $\mathfrak{S}_{k}$-module and $C^{\prime \prime} \in \operatorname{Con}\left(W_{n-k}\right)$. Using 22.5 (a) we have 
$E=\mathbf{j}_{\mathfrak{S}_{k^{\prime}} \times \mathfrak{S}_{k^{\prime \prime}}}^{\mathfrak{S}_{k}}\left(\operatorname{sgn} \otimes E^{\prime}\right)$ where $k^{\prime}+k^{\prime \prime}=k, k^{\prime}>0$ and $E^{\prime}$ is a simple $\mathfrak{S}_{k^{\prime \prime}}$-module. Let $\tilde{C}=\mathbf{j}_{\mathfrak{S}_{k^{\prime \prime}} \times W_{n-k}}^{W_{n-k^{\prime}}}\left(E^{\prime} \otimes C^{\prime}\right) \in \operatorname{Con}\left(W_{n-k^{\prime}}\right)$. Then $C=\mathbf{j}_{\mathfrak{S}_{k^{\prime}} \times W_{n-k^{\prime}}}^{W}\left(\operatorname{sgn}_{k^{\prime}} \otimes \tilde{C}\right)$. By the induction hypothesis, $\tilde{C}$ is of the form described in (a). Using an argument as in (A) or (C) we deduce that $C$ is of the form described in (a). The proposition is proved.

Proposition 22.25. Assume that $b^{\prime}>0$.

(a) Let $E \in \operatorname{Irr} W$. Then $E \in \operatorname{Con}(W)$.

(b) All constructible representations of $W$ are obtained as in (a).

We prove (a). We may assume that $E=[\Lambda]$ where $\Lambda \in \mathrm{Sy}_{a, b ; n}^{N}$ does not contain both 0 and $b^{\prime}$. We argue by induction on $n$. If $n=0$ the result is clear. Assume now that $n \geq 1$.

(A) Assume that either (1) there exist two entries $z, z^{\prime}$ of $\Lambda$ such that $z^{\prime}-z>a$ and there is no entry $z^{\prime \prime}$ of $\Lambda$ such that $z<z^{\prime \prime}<z^{\prime}$, or (2) there exists an entry $z^{\prime}$ of $\Lambda$ such that $z^{\prime} \geq a$ and there is no entry $z^{\prime \prime}$ of $\Lambda$ such that $z^{\prime \prime}<$ $z^{\prime}$. Let $\Lambda^{\prime}$ be the symbol obtained from $\Lambda$ by substracting $a$ from each entry $\tilde{z}$ of $\Lambda$ such that $\tilde{z} \geq z^{\prime}$ and leaving the other entries of $\Lambda$ unchanged. Then $\Lambda^{\prime} \in \operatorname{Sy}_{a, b ; n-k}^{N}$ with $n-k<n$. By the induction hypothesis, $\left[\Lambda^{\prime}\right] \in \operatorname{Con}\left(W_{n-k}\right)$. Since, by 22.5 , the sign representation $\operatorname{sgn}_{k}$ of $\mathfrak{S}_{k}$ is constructible, it follows that $\operatorname{sgn}_{k} \otimes\left[\Lambda^{\prime}\right] \in \operatorname{Con}\left(\mathfrak{S}_{k} \times W_{n-k}\right)$. Using 22.17 , we have $\mathbf{j}_{\mathfrak{S}_{k} \times W_{n-k}}^{W}\left(\operatorname{sgn}_{k} \otimes\left[\Lambda^{\prime}\right]\right)=[\Lambda]$ hence $[\Lambda] \in \operatorname{Con}(W)$.

(B) Assume that there exist two entries $z, z^{\prime}$ of $\Lambda$ such that $0<z^{\prime}-z<a$. Let $t$ be the smallest integer such that at $+b^{\prime} \geq \lambda_{i}$ for all $i \in[1, N+r]$ and at $\geq \mu_{j}$ for all $j \in[1, N]$. Let $\bar{\Lambda} \in \mathrm{Sy}_{a, b ; n}^{t+N-r}$ be as in 22.8 with respect to this $t$. Then $\bar{\Lambda}$ does not contain both 0 and $b^{\prime}$. Now (A) is applicable to $\bar{\Lambda}$. Hence $[\bar{\Lambda}] \in \operatorname{Con}(W)$. By 22.18 we have $[\bar{\Lambda}] \otimes \operatorname{sgn}=[\Lambda]$ hence $[\Lambda] \in \operatorname{Con}(W)$.

(C) Assume that we are not in case (A) and not in case (B). Then the entries of $\Lambda$ are either $0, a, 2 a, \ldots, t a$ or $b^{\prime}, a+b^{\prime}, 2 a+b^{\prime}, \ldots, t a+b^{\prime}$. This cannot happen for $n \geq 1$. This proves (a).

The proof of (b) is entirely similar to that of 22.24(b). The proposition is proved.

22.26. We now assume that $n \geq 2$ and that $W^{\prime}=W_{n}^{\prime}$ is the kernel of $\chi_{n}: W_{n} \rightarrow$ \pm 1 in 22.10. We regard $W_{n}^{\prime}$ as a Coxeter group with generators $s_{1}, s_{2}, \ldots, s_{n-1}$ as in 22.9 and $s_{n}^{\prime}=\left(n-1, n^{\prime}\right)\left((n-1)^{\prime}, n\right)$ (product of transpositions). Let $L: W^{\prime} \rightarrow \mathbf{N}$ be the weight function given by $L(w)=a l(w)$ for all $w$. Here $a>0$.

For $\Lambda \in \mathrm{Sy}_{a, 0}^{N}$ we denote by $\Lambda^{\text {tr }}$ the symbol whose first (resp. second) row is the second (resp. first) row of $\Lambda$. We then have $\Lambda^{t r} \in \mathrm{Sy}_{a, 0}^{N}$. From the definitions we see that the simple $W_{n}$-modules $[\Lambda],\left[\Lambda^{t r}\right]$ have the same restriction to $W^{\prime}$; this restriction is a simple $W^{\prime}$-module $[\underline{\Lambda}]$ if $\Lambda \neq \Lambda^{\text {tr }}$ and is a direct sum of two non-isomorphic simple $W^{\prime}$-modules $\left.\left[{ }^{I} \underline{\Lambda}\right],{ }^{I I} \underline{\Lambda}\right]$ if $\Lambda=\Lambda^{\operatorname{tr}}$. In this way we see that

the simple $W^{\prime}$-modules are naturally in bijection with the set of orbits of the 
involution of $\mathrm{Sy}_{a, 0 ; n}$ induced by $\Lambda \mapsto \Lambda^{\text {tr }}$ except that each fixed point of this involution corresponds to two simple $W^{\prime}$-modules.

Let $\tilde{Z} \in \mathcal{M}_{a, 0 ; n}^{N}$. Let $Z$ be the set of singles of $\tilde{Z}$. Assume first that $Z \neq \emptyset$. Each set $Y \in \underline{Z}_{0}$ gives rise to a symbol $\Lambda_{Y}$ in $\mathrm{Sy}_{a, 0 ; n}^{N}$ : the first row of $\Lambda_{Y}$ consists of $Z-Y$ and one element in each double of $\tilde{Z}$; the second row consists of $Y$ and one element in each double of $\tilde{Z}$. For any $\iota \in \operatorname{Inv}_{0}(Z)$ we define $c(\tilde{Z}, \iota) \in \operatorname{Mod} W$ by

$$
c(\tilde{Z}, \iota) \oplus c(\tilde{Z}, \iota)=\oplus_{Y \in \mathcal{S}_{\iota}}\left[\Lambda_{Y}\right] \in \operatorname{Mod} W .
$$

Note that $Y$ and $Z-Y$ have the same contribution to the sum. A proof entirely similar to that of 22.24 shows that $c(\tilde{Z}, \iota) \in \operatorname{Con}(W)$. Moreover, if $Z=\emptyset$ and $\Lambda=$ $\Lambda^{t r} \in \operatorname{Sy}_{a, 1 ; n}^{N}$ is defined by $\pi_{N}(\Lambda)=\tilde{Z}$, then $\left[{ }^{I} \underline{\Lambda}\right] \in \operatorname{Con}(W)$ and $\left[{ }^{I I} \underline{\Lambda}\right] \in \operatorname{Con}(W)$. All constructible representations of $W$ are obtained in this way.

22.27. Assume that $W$ is of type $F_{4}$ and that the values of $L: W \rightarrow \mathbf{N}$ on $S$ are $a, a, b, b$ where $a>b>0$.

Case 1. Assume that $a=2 b$. There are four simple $W$-modules $\rho_{1}, \rho_{2}, \rho_{8}, \rho_{9}$ (subscript equals dimension) with $\mathbf{a}=3 b$. Then

$$
\rho_{1} \oplus \rho_{2}, \rho_{1} \oplus \rho_{8}, \rho_{2} \oplus \rho_{9}, \rho_{8} \oplus \rho_{9} \in \operatorname{Con}(W) .
$$

(They are obtained by $\mathbf{j}$ from the $W_{I}$ of type $B_{3}$ with parameters $a, b, b$.)

The simple $W$-modules $\rho_{1}^{\dagger}, \rho_{2}^{\dagger}, \rho_{8}^{\dagger}, \rho_{9}^{\dagger}$ have $\mathbf{a}=15 b$ and

$$
\rho_{1}^{\dagger} \oplus \rho_{2}^{\dagger}, \rho_{1}^{\dagger} \oplus \rho_{8}^{\dagger}, \rho_{2}^{\dagger} \oplus \rho_{9}^{\dagger}, \rho_{8}^{\dagger} \oplus \rho_{9}^{\dagger} \in \operatorname{Con}(W) \text {. }
$$

There are five simple $W$-modules $\rho_{12}, \rho_{16}, \rho_{6}, \rho_{6}^{\prime}, \rho_{4}$ (subscript equals dimension) with $\mathbf{a}=7 b$. Then

$$
\rho_{4} \oplus \rho_{16}, \rho_{12} \oplus \rho_{16} \oplus \rho_{6}, \rho_{12} \oplus \rho_{16} \oplus \rho_{6}^{\prime} \in \operatorname{Con}(W) .
$$

All 12 simple $W$-modules other than the 13 listed above, are constructible. All constructible representations of $W$ are thus obtained.

Case 2. Assume that $a \notin\{b, 2 b\}$. The simple $W$-modules $\rho_{12}, \rho_{16}, \rho_{6}, \rho_{6}^{\prime}, \rho_{4}$ in Case 1 now have $\mathbf{a}=3 a+b$ and

$$
\rho_{4} \oplus \rho_{16}, \rho_{12} \oplus \rho_{16} \oplus \rho_{6}, \rho_{12} \oplus \rho_{16} \oplus \rho_{6}^{\prime} \in \operatorname{Con}(W)
$$

All 20 simple $W$-modules other than the 5 listed above, are constructible. All constructible representations of $W$ are thus obtained.

22.28. Assume that $W$ is of type $G_{2}$ and that the values of $L: W \rightarrow \mathbf{N}$ on $S$ are $a, b$ where $a>b>0$. Let $\rho_{2}, \rho_{2}^{\prime}$ be the two 2-dimensional simple $W$-modules. They have $\mathbf{a}=a$ and $\rho_{2} \oplus \rho_{2}^{\prime}$ is constructible. All 4 simple $W$-modules other than the 2 listed above, are constructible. All constructible representations of $W$ are thus obtained. 
22.29. Let $\mathcal{L}$ be the set of all weight functions $L: W \rightarrow \mathbf{N}$ such that $L(s)>0$ for all $s \in S$. We assume that $P 1-P 15$ in $\S 14$ hold for any $L \in \mathcal{L}$. For $L, L^{\prime} \in \mathcal{L}$ we write $L \sim L^{\prime}$ if the constructible representations of $W$ with respect to $L$ are the same as those with respect to $L^{\prime}$. This is an equivalence relation on $\mathcal{L}$. From the results in this section we see that any equivalence class for $\sim$ contains some $L$ which is attached to some $(G, F, \mathcal{P}, \mathbf{E})$ as in 0.3 .

We expect that the constructible representations of $W$ are exactly the representations of $W$ carried by the left cells of $W$ (for fixed $L \in \mathcal{L}$ ). (For $L=l$ this holds by [L7]. For $W$ of type $F_{4}$ and general $L$ this holds by [G].) This would imply that for $L, L^{\prime} \in \mathcal{L}$ we have $L \sim L^{\prime}$ if and only if the representations of $W$ carried by the left cells of $W$ with respect to $L$ are the same as those with respect to $L^{\prime}$.

\section{TWO-SIDED CELLS}

23.1. We define a graph $\mathcal{G}_{W}$ as follows. The vertices of $\mathcal{G}_{W}$ are the simple $W$ modules up to isomorphism. Two non-isomorphic simple $W$-modules are joined in $\mathcal{G}_{W}$ if they both appear as components of some constructible representation of $W$. Let $\frac{\mathcal{G}_{W}}{\sim}$ be the set of connected components of $\mathcal{G}_{W}$. The connected components of $\mathcal{G}_{W}$ are determined explicitly by the results in $\S 22$ for $W$ irreducible.

For example, in the setup of $22.4,22.5$ we have $\mathcal{G}_{W}=\frac{\mathcal{G}_{W}}{\sim}$. In the setup of 22.24, $\frac{\mathcal{G}_{W}}{\sim}$ is naturally in bijection with $\mathcal{M}_{a, b ; n}$. (Here, 22.22 is used). In the setup of 22.25 , we have $\mathcal{G}_{W}=\frac{\mathcal{G}_{W}}{\sim}$.

We show that:

(a) if $E, E^{\prime}$ are in the same connected component of $\mathcal{G}_{W}$ then $E \sim_{\mathcal{L R}} E^{\prime}$.

We may assume that both $E, E^{\prime}$ appear in some constructible representation of $W$. By 22.2 , there exists a left cell $\Gamma$ such that $[E:[\Gamma]] \neq 0,\left[E^{\prime}:[\Gamma]\right] \neq 0$. By 21.2 , we have $\left[E_{\boldsymbol{\sim}}: J_{\mathbf{C}}^{\Gamma}\right] \neq 0,\left[E_{\boldsymbol{\uparrow}}^{\prime}: J_{\mathbf{C}}^{\Gamma}\right] \neq 0$. Hence $E \sim_{\mathcal{L R}} E^{\prime}$, as desired.

23.2. Let $c_{W}$ be the set of two-sided cells of $W, L$. Consider the (surjective) map $\operatorname{Irr} W \rightarrow c_{W}$ which to $E$ associates the two-sided cell $\mathbf{c}$ such that $E \sim_{\mathcal{L R}} x$ for $x \in \mathbf{c}$. By 23.1 this induces a (surjective) map

(a) $\omega_{W}: \frac{\mathcal{G}_{W}}{\sim} \rightarrow c_{W}$.

We conjecture that $\omega_{W}$ is a bijection. This is made plausible by:

Proposition 23.3. Assume that $W, L$ is split. Then $\omega_{W}$ is a bijection.

Let $E, E^{\prime} \in \operatorname{Irr} W$ be such that $E \sim_{\mathcal{L R}} E^{\prime}$. By 22.3, we can find constructible representations $C, C^{\prime}$ such that $[E: C] \neq 0,\left[E^{\prime}: C^{\prime}\right] \neq 0$. By 22.2, we can find left cells $\Gamma, \Gamma^{\prime}$ such that $C=[\Gamma], C^{\prime}=\left[\Gamma^{\prime}\right]$. Then $[E:[\Gamma]] \neq 0,\left[E^{\prime}:\left[\Gamma^{\prime}\right]\right] \neq 0$. Let $d \in \mathcal{D} \cap \Gamma, d^{\prime} \in \mathcal{D} \cap \Gamma^{\prime}$. Since $\gamma_{d}=[\Gamma]$ and $\left[E:[\Gamma] \neq 0\right.$, we have $E \sim_{\mathcal{L R}} d$. Similarly, $E^{\prime} \sim_{\mathcal{L R}} d^{\prime}$. Hence $d^{\prime} \sim_{\mathcal{L R}} d^{\prime}$. By 18.4(c), there exists $u \in W$ such that $t_{d} t_{u} t_{d^{\prime}} \neq 0$. (Here we use the splitness assumption.) Note that $j \mapsto j t_{u} t_{d^{\prime}}$ is a $J_{\mathbf{C}}$-linear map $J_{\mathbf{C}}^{\Gamma} \rightarrow J_{\mathbf{C}}^{\Gamma^{\prime}}$. This map is non-zero since it takes $t_{d}$ to $t_{d} t_{u} t_{d^{\prime}} \neq 0$. Thus, $\operatorname{Hom}_{J_{\mathbf{C}}}\left(J_{\mathbf{C}}^{\Gamma}, J_{\mathbf{C}}^{\Gamma^{\prime}}\right) \neq 0$. Using 21.2, we deduce that $\operatorname{Hom}_{W}\left([\Gamma],\left[\Gamma^{\prime}\right]\right) \neq 0$. Hence there exists $\tilde{E} \in \operatorname{Irr} W$ such that $\tilde{E}$ is a component of both $[\Gamma]=C$ and 
$\left[\Gamma^{\prime}\right]=C^{\prime}$. Thus, both $E, \tilde{E}$ appear in $C$ and both $\tilde{E}, E^{\prime}$ appear in $C^{\prime}$. Hence $E, E^{\prime}$ are in the same connected component of $\mathcal{G}_{W}$. The proposition is proved.

23.4. Assume now that $W, S, L, \tilde{W}, u$ are as in $16.1,16.3$ and $\tilde{W}$ is an irreducible Weyl group.

Let $c_{\tilde{W}}^{!}$be the set of all $u$-stable two-sided cells of $\tilde{W}$. Let $c_{\tilde{W}}^{\star}$ be the set of all two-sided cells of $\tilde{W}$ which meet $W$. We have $c_{\tilde{W}}^{\star} \subset c_{\tilde{W}}^{!} \subset c_{\tilde{W}}$. Let $f: c_{W} \rightarrow c_{\tilde{W}}^{\star}$ be the map which attaches to a two-sided cell of $W$ the unique two-sided cell of $\tilde{W}$ containing it; this map is well defined by $16.13(\mathrm{a})$ and is obviously surjective.

Proposition 23.5. In the setup of 23.4, $\omega_{W}$ is a bijection and $f: c_{W} \rightarrow c_{\tilde{W}}^{\star}$ is a bijection.

Since $\omega_{W}, f$ are surjective, the composition $f \omega_{W}: \frac{\mathcal{G}_{W}}{\sim} \rightarrow c_{\tilde{W}}^{\star}$ is surjective. Hence it is enough to show that this composition is injective. For this it suffices to check one of the two statements below:

(a) $\sharp\left(\frac{\mathcal{G}_{W}}{\sim}\right)=\sharp\left(c_{\tilde{W}}^{\star}\right)$;

(b) the composition $\frac{\mathcal{G}_{W}}{\sim} \stackrel{f \omega_{W}}{\longrightarrow} c_{\tilde{W}}^{\star} \subset c_{\tilde{W}} \stackrel{f^{\prime}}{\longrightarrow} \mathbf{N} \oplus \mathbf{N}\left(\right.$ where $f^{\prime}(\mathbf{c})=\left(\mathbf{a}(x), \mathbf{a}\left(x w_{0}\right)\right)$ for $x \in \mathbf{c}$ ) is injective.

Note that the value of the composition (b) at $E$ is $\left(\mathbf{a}_{E}, \mathbf{a}_{E^{\dagger}}\right)$.

Case 1 . $W$ is of type $G_{2}$ and $\tilde{W}$ is of type $D_{4}$. Then (b) holds: the composition (b) takes distinct values $(0,12),(1,7),(3,3),(7,1),(12,0)$ on the 5 elements of $\frac{\mathcal{G}_{W}}{\sim}$.

Case 2. $W$ is of type $F_{4}$ and $\tilde{W}$ is of type $E_{6}$. Then again (b) holds.

Case 3. $W$ is of type $B_{n}$ with $n \geq 2$ and $\tilde{W}$ is of type $A_{2 n}$ or $A_{2 n+1}$. Then $u$ is conjugation by the longest element $\tilde{w}_{0}$ of $\tilde{W}$. We show that (a) holds.

Let $Y$ be the set of all $E \in \operatorname{Irr} \tilde{W}$ (up to isomorphism) such that $\operatorname{tr}\left(\tilde{w}_{0}, E\right) \neq 0$. Let $Y^{\prime}$ be the set of all $E^{\prime} \in \operatorname{Irr} W$ (up to isomorphism). By 23.4 and 23.1 we have a natural bijection between $c_{\tilde{W}}$ and the set of isomorphism classes of $E \in \operatorname{Irr} \tilde{W}$. If $\mathbf{c} \in c_{\tilde{W}}$ corresponds to $E$, then the number of fixed points of $u$ on $\mathbf{c}$ is clearly $\pm \operatorname{dim}(E) \operatorname{tr}\left(\tilde{w}_{0}, E\right)$. Hence $\sharp\left(c_{\tilde{W}}^{\star}\right)=\sharp Y$. From 23.1 we have $\sharp\left(\frac{\mathcal{G}_{W}}{\sim}\right)=\sharp Y$. Hence to show (a) it suffices to show that $\sharp Y=\sharp Y^{\prime}$. But this is shown in [L3].

Case 4. Assume that $\tilde{W}$ is of type $D_{n}$ and $W$ is of type $B_{n-1}$ with $n \geq 3$. We will show that (a) holds. We change notation and write $W^{\prime}$ instead of $\tilde{W}, W^{\prime u}$ instead of $W$. Then $W^{\prime}$ is as in 22.26 and we may assume that $u: W^{\prime} \rightarrow W^{\prime}$ is conjugation by $s_{n}$ (as in 22.26). Let $\mathcal{M}_{1,0 ; n}^{N, !}$ be the set of all elements in $\mathcal{M}_{1,0 ; n}^{N}$ whose set of singles is non-empty. Let

$$
\mathcal{M}_{1,0 ; n}^{!}=\lim _{N \rightarrow \infty} \mathcal{M}_{1,0 ; n}^{N, !}
$$

By 22.26 and 23.3, $c_{W^{\prime}}^{!}$is naturally in bijection with $\mathcal{M}_{1,0 ; n}^{!}$. By 23.1, $\frac{\mathcal{G}_{W^{\prime} u}}{\sim}$ is naturally in bijection with $\mathcal{M}_{1,2 ; n-1}$. The identity map is clearly a bijection $\mathcal{M}_{1,2 ; n-1}^{N} \stackrel{\sim}{\longrightarrow} \mathcal{M}_{1,0 ; n}^{N+1, !}$. It induces a bijection $\mathcal{M}_{1,2 ; n-1} \stackrel{\sim}{\longrightarrow} \mathcal{M}_{1,0 ; n}^{!}$. Hence to prove 
that $\sharp\left(\frac{\mathcal{G}_{W^{\prime} u}}{\sim}\right) \leq \sharp\left(c_{W^{\prime}}^{\star}\right)$ it suffices to prove that $\sharp\left(\mathcal{M}_{1,0 ; n}^{!}\right)=\sharp\left(\mathcal{M}_{1,0 ; n}^{\star}\right)$. In other words, we must show that

(c) any u-stable two-sided cell of $W^{\prime}$ meets $W^{\prime u}$.

Now 22.26 and 23.3 provide an inductive procedure to obtain any $u$-stable twosided cell of $W^{\prime}$. Namely such a cell is obtained by one of two procedures:

(i) we consider a $u$-stable two-sided cell in a parabolic subgroup of type $\mathfrak{S}_{k} \times$ $D_{n-k}$ (where $\left.n-k \in[2, n-1]\right)$ and we attach to it the unique two-sided cell of $W^{\prime}$ that contains it;

(ii) we take a two-sided cell obtained in (i) and multiply it on the right by the longest element of $W^{\prime}$.

Since we may assume that (c) holds when $n$ is replaced by $n-k \in[2, n-1]$, we see that the procedures (i) and (ii) yield only two-sided cells that contain $u$-fixed elements. This proves (c). The proposition is proved.

\section{VirTuAl CELlS}

24.1. In this section we preserve the setup of 20.1 .

A virtual cell of $W$ (with respect to $L: W \rightarrow \mathbf{N}$ ) is an element of $K(W)$ of the form $\gamma_{x}$ (see 20.16) for some $x \in W$.

Lemma 24.2. Let $x \in W$ and let $\Gamma$ be the left cell containing $x$.

(a) If $\gamma_{x} \neq 0$ then $x \in \Gamma \cap \Gamma^{-1}$.

(b) $\gamma_{x}$ is a C-linear combination of $E \in \operatorname{Irr} W$ such that $[E:[\Gamma]] \neq 0$.

Assume that $\gamma_{x} \neq 0$. Then there exists $\mathcal{E} \in \operatorname{Irr} J_{\mathbf{C}}$ such that $\operatorname{tr}\left(t_{x}, \mathcal{E}\right) \neq 0$. We have $\mathcal{E}=\oplus_{d \in \mathcal{D}} t_{d} \mathcal{E}$ and $t_{x}: \mathcal{E} \rightarrow \mathcal{E}$ maps the summand $t_{d} \mathcal{E}$ (where $x \sim_{\mathcal{L}} d$ ) into $t_{d^{\prime}}$, where $d^{\prime} \sim_{\mathcal{L}} x^{-1}$ and all other summands to 0 . Since $\operatorname{tr}\left(t_{x}, \mathcal{E}\right) \neq 0$, we must have $t_{d} \mathcal{E}=t_{d^{\prime}} \mathcal{E} \neq 0$ hence $d=d^{\prime}$ and $x \sim_{\mathcal{L}} x^{-1}$. This proves (a).

We prove (b). Let $d \in \mathcal{D} \cap \Gamma$. Assume that $E \in \operatorname{Irr} W$ appears with $\neq 0$ coefficient in $\gamma_{x}$. Then $\operatorname{tr}\left(t_{x}, E_{\boldsymbol{\omega}}\right) \neq 0$. As we have seen in the proof of (a), we have $t_{d} E_{\boldsymbol{\phi}} \neq 0$. Using 21.3,21.2, we deduce $\left[E_{\boldsymbol{\phi}}: J_{\mathbf{C}}^{\Gamma}\right] \neq 0$ and $\left.\left[E_{\boldsymbol{\phi}}:[\Gamma]\right]_{\mathbf{\phi}}\right] \neq 0$. Hence $[E:[\Gamma]] \neq 0$. The lemma is proved.

24.3. In the remainder of this section we will give a number of explicit computations of virtual cells.

Lemma 24.4. In the setup of 22.10, $w_{0}$ acts on $[\Lambda]$ as multiplication by $\epsilon_{[\Lambda]}=(-1)^{\sum_{j}\left(a^{-1} \mu_{j}-j+1\right)}$.

Using the definitions we are reduced to the case where $k=n$ or $l=n$. If $k=n$ we have $\epsilon_{[\Lambda]}=1$ since $[\Lambda]$ factors through $\mathfrak{S}_{n}$ and the longest element of $W_{n}$ is in the kernel of $W_{n} \rightarrow \mathfrak{S}_{n}$. Similarly, if $l=n$ we have $\epsilon_{[\Lambda]}=\epsilon_{\chi_{n}}=(-1)^{n}$. The lemma is proved.

Proposition 24.5. Assume that we are in the setup of 22.23. Let $\iota \in \operatorname{Inv}_{r}(Z)$ and let $\kappa: \mathcal{S}_{\iota} \rightarrow \mathbf{F}_{2}$ be an affine-linear function. Let 


$$
c(\tilde{Z}, \iota, \kappa)=\sum_{Y \in \mathcal{S}_{\iota}}(-1)^{\kappa(Y)}\left[\Lambda_{Y}\right] \in K(W) .
$$

There exists $x \in W$ such that $\gamma_{x}= \pm c(\tilde{Z}, \iota, \kappa)$.

To some extent the proof is a repetition of the proof of $22.24(\mathrm{a})$, but we have to keep track of $\kappa$, a complicating factor.

We argue by induction on the rank $n$ of $\tilde{Z}$. If $n=0$ the result is clear. Assume now that $n \geq 1$. We may assume that 0 is not a double of $\tilde{Z}$. Let at be the largest entry of $\tilde{Z}$.

(A) Assume that there exists $i, 0 \leq i<t$, such that ai does not appear in $\tilde{Z}$. Then $\tilde{Z}$ is obtained from a multiset $\tilde{Z}^{\prime}$ of rank $n-k<n$ by increasing each of the $k$ largest entries by $a$ and this set of largest entries is unambiguously defined. The set $Z^{\prime}$ of singles of $\tilde{Z}^{\prime}$ is naturally in bijection with $Z$.

Let $\iota^{\prime}, \kappa^{\prime}$ correspond to $\iota, \kappa$ under this bijection. By the induction hypothesis, there exists $x^{\prime} \in W_{n-k}$ such that $\gamma_{x^{\prime}}^{W_{n-k}}= \pm c\left(\tilde{Z}^{\prime}, \iota^{\prime}, \kappa^{\prime}\right) \in K\left(W_{n-k}\right)$. Let $w_{0, k}$ be the longest element of $\mathfrak{S}_{k}$. Then

$$
\gamma_{w_{0, k} x^{\prime}}^{\mathfrak{S}_{k} \times W_{n-k}}=\gamma_{w_{0, k}}^{\mathfrak{S}_{k}} \otimes \gamma_{x^{\prime}}^{W_{n-k}}=\operatorname{sgn}_{k} \otimes \gamma_{x^{\prime}}^{W_{n-k}}
$$

and

$$
\gamma_{w_{0, k} x^{\prime}}^{W}=\mathbf{j}_{\mathfrak{S}_{k} \times W_{n-k}}^{W}\left(\gamma_{w_{0, k} x^{\prime}}^{\mathfrak{S}_{k} \times W_{n-k}}\right)
$$

$$
=\mathbf{j}_{\mathfrak{S}_{k} \times W_{n-k}}^{W}\left(\operatorname{sgn}_{k} \otimes \gamma_{x^{\prime}}^{W_{n-k}}\right)= \pm \mathbf{j}_{\mathfrak{S}_{k} \times W_{n-k}}^{W}\left(\operatorname{sgn}_{k} \otimes c\left(\tilde{Z}^{\prime}, \iota^{\prime}, \kappa^{\prime}\right)\right)= \pm c(\tilde{Z}, \iota, \kappa),
$$

as required.

(B) Assume that there exists $i, 0<i \leq t$ such that $a i$ is a double of $\tilde{Z}$. Let $\bar{Z}$ be as in 22.8 (with respect to our $t$ ). Then 0 is not a double of $\overline{\tilde{Z}}$ and the largest entry of $\bar{Z}$ is at. Let $\bar{Z}$ be the set of singles of $\overline{\tilde{Z}}$. We have $\bar{Z}=\{a t-z ; z \in Z\}$. Thus $\bar{Z}, Z$ are naturally in (order reversing) bijection under $j \mapsto a t-j$. Let $\iota^{\prime} \in \operatorname{Inv}_{r}(\bar{Z})$ correspond to $\iota$ under this bijection and let $\kappa^{\prime}: \mathcal{S}_{\iota^{\prime}} \rightarrow \mathbf{F}_{2}$ correspond to $\kappa$ under this bijection. Define $\kappa^{\prime \prime}: \mathcal{S}_{\iota^{\prime}} \rightarrow \mathbf{F}_{2}$ by $\kappa^{\prime \prime}(Y)=\kappa^{\prime}(Y)+\sum_{y \in Y} a^{-1} y$ (an affine-linear function). Since $a t-a i$ does not appear in $\overline{\tilde{Z}},(\mathrm{~A})$ is applicable to $\overline{\tilde{Z}}$. Hence there exists $x^{\prime} \in W$ such that $\gamma_{x^{\prime}}= \pm c\left(\overline{\tilde{Z}}, \iota^{\prime}, \kappa^{\prime \prime}\right)$. By 20.23, 22.18, 24.4 , we have

$\gamma_{x^{\prime} w_{0}}=(-1)^{l\left(x^{\prime}\right)} \zeta\left(\gamma_{x^{\prime}}\right)= \pm \zeta\left(c\left(\overline{\tilde{Z}}, \iota^{\prime}, \kappa^{\prime \prime}\right)\right) \otimes \operatorname{sgn}= \pm c\left(\overline{\tilde{Z}}, \iota^{\prime}, \kappa^{\prime}\right) \otimes \operatorname{sgn}= \pm c(\tilde{Z}, \iota, \kappa)$,

as desired.

(C) Assume that we are not in case (A) and not in case (B). Then $\tilde{Z}=$ $\{0, a, 2 a, \ldots, t a\}=Z$. We can find $i a,(i+1) a$ in $Z$ such that $\iota$ interchanges $i a,(i+1) a$ and induces on $Z-\{i a,(i+1) a\}$ an $r$-admissible involution $\iota_{1}$. 
(C1) Assume first that $\kappa(Y)=\kappa(Y *\{i a,(i+1) a\})$ for any $Y \in \mathcal{S}_{\iota} . \quad(*$ is symmetric difference.) Let

$$
\tilde{Z}^{\prime}=\{0, a, 2 a, \ldots, i a, i a,(i+1) a,(i+2) a, \ldots,(t-1) a\}
$$

This has rank $n-k<n$. The set of singles of $\tilde{Z}^{\prime}$ is

$$
Z^{\prime}=\{0, a, 2 a, \ldots,(i-1) a,(i+1) a, \ldots,(t-1) a\}
$$

It is in natural (order preserving) bijection with $Z-\{i a,(i+1) a\}$. Hence $\iota_{1}$ induces $\iota^{\prime} \in \operatorname{Inv}_{r}\left(Z^{\prime}\right)$. We have an obvious surjective map of affine spaces $\pi: \mathcal{S}_{\iota} \rightarrow \mathcal{S}_{\iota^{\prime}}$ and $\kappa$ is constant on the fibres of this map. Hence there is an affine-linear map $\kappa^{\prime}: \mathcal{S}_{\iota^{\prime}} \rightarrow \mathbf{F}_{2}$ such that $\kappa=\kappa^{\prime} \pi$. By the induction hypothesis, there exists $x^{\prime} \in W_{n-k}$ such that $\gamma_{x^{\prime}}^{W_{n-k}}= \pm c\left(\tilde{Z}^{\prime}, \iota^{\prime}, \kappa^{\prime}\right) \in K\left(W_{n-k}\right)$. Let $w_{0, k}$ be the longest element of $\mathfrak{S}_{k}$. Then (a), (b) hold and we are done.

(C2) Assume next that $\kappa(Y) \neq \kappa(Y *\{i a,(i+1) a\})$ for some (or equivalently any) $Y \in \mathcal{S}_{\iota}$. We have

$$
\overline{\tilde{Z}}=\{0,0, a, a, 2 a, 2 a, \ldots, t a, t a\}-\{a t-0, a t-a, \ldots, a t-a t\}=\tilde{Z}=Z .
$$

Let $\iota^{\prime} \in \operatorname{Inv}_{r}(Z)$ correspond to $\iota$ under the bijection $z \mapsto t a-z$ of $Z$ with itself; let $\kappa^{\prime}: \mathcal{S}_{\iota^{\prime}} \rightarrow \mathbf{F}_{2}$ correspond to $\kappa$ under this bijection. Let $\kappa^{\prime \prime}: \mathcal{S}_{\iota^{\prime}} \rightarrow \mathbf{F}_{2}$ be given by $\kappa^{\prime \prime}(Y)=\kappa^{\prime}(Y)+\sum_{y \in Y} a^{-1} y$ (an affine-linear function). Note that $\iota^{\prime}$ interchanges $(t-i-1) a,(t-i) a$ and induces on $Z-\{(t-i-1) a,(t-i) a\}$ an $r$-admissible involution. We show that for any $Y \in \mathcal{S}_{\iota^{\prime}}$ we have

$$
\kappa^{\prime \prime}(Y)=\kappa^{\prime \prime}(Y *\{(t-i-1) a,(t-i) a\})
$$

or equivalently

$$
\kappa^{\prime}(Y)=\kappa^{\prime}(Y *\{(t-i-1) a,(t-i) a\})+1 .
$$

This follows from our assumption $\kappa(Y)=\kappa(Y *\{i a,(i+1) a\})+1$ for any $Y \in \mathcal{S}_{\iota}$. We see that case $(\mathrm{C} 1)$ applies to $\iota^{\prime}, \kappa^{\prime \prime}$ so that there exists $x^{\prime} \in W$ with $\gamma_{x^{\prime}}=$ $\pm c\left(\tilde{Z}, \iota^{\prime}, \kappa^{\prime \prime}\right)$. By $20.23,22.18,24.4$, we have

$\gamma_{x^{\prime} w_{0}}=(-1)^{l\left(x^{\prime}\right)} \zeta\left(\gamma_{x^{\prime}}\right)= \pm \zeta\left(c\left(\overline{\tilde{Z}}, \iota^{\prime}, \kappa^{\prime \prime}\right)\right) \otimes \operatorname{sgn}= \pm c\left(\overline{\tilde{Z}}, \iota^{\prime}, \kappa^{\prime}\right) \otimes \operatorname{sgn}= \pm c(\tilde{Z}, \iota, \kappa)$,

as desired. The proposition is proved.

24.6. Assume that we are in the setup of 22.27. By 22.27,

$$
\rho_{4}+\rho_{16}, \rho_{12}+\rho_{16}+\rho_{6}, \rho_{12}+\rho_{16}+\rho_{6}^{\prime}
$$

are constructible, hence (by $22.2,21.4$ ) are of the form $n_{d} \gamma_{d}$ for suitable $d \in \mathcal{D}$, hence are \pm virtual cells.

Let $d \in \mathcal{D}$ be such that $n_{d} \gamma_{d}=\rho_{12}+\rho_{16}+\rho_{6}$. Let $\Gamma$ be the left cell that contains $d$. Recall (21.4) that $[\Gamma]=A \oplus B \oplus C$ where $A=\rho_{12}, B=\rho_{16}, C=\rho_{6}$. By 
the discussion in 21.10 we see that $J_{\mathbf{C}}^{\Gamma \cap \Gamma^{-1}}$ has exactly three simple modules (up to isomorphism), namely $t_{d} A_{\mathbf{s}}, t_{d} B_{\boldsymbol{\phi}}, t_{d} C_{\mathbf{s}}$, and these are 1-dimensional. Since $J^{\Gamma \cap \Gamma^{-1}}$ is a semisimple algebra (21.9), it follows that it is commutative of dimension 3. Hence $\Gamma \cap \Gamma^{-1}$ consists of three elements $d, x, y$. Let $p_{A}, p_{B}, p_{C}$ denote the traces of $t_{x}$ on $A_{\boldsymbol{\mathbf { p }}}, B_{\boldsymbol{\mathbf { }}}, C_{\boldsymbol{\mathbf { }}}$ respectively. Let $q_{A}, q_{B}, q_{C}$ denote the traces of $t_{y}$ on $A_{\mathbf{\uparrow}}, B_{\mathbf{\uparrow}}, C_{\mathbf{\wedge}}$ respectively. By $20.24, p_{A}, p_{B}, p_{C}, q_{A}, q_{B}, q_{C}$ are integers. Recall that

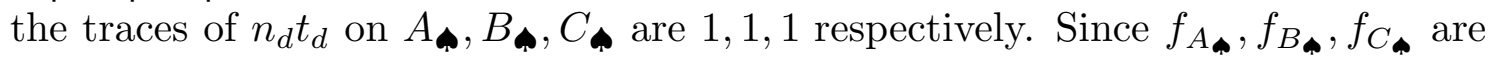
$6,2,3$ we see that the orthogonality formula 21.10 gives

$$
\begin{gathered}
1+p_{A}^{2}+q_{A}^{2}=6,1+p_{B}^{2}+q_{B}^{2}=2,1+p_{C}^{2}+q_{C}^{2}=3 \\
1+p_{A} p_{B}+q_{A} q_{B}=0,1+p_{A} p_{C}+q_{A} q_{C}=0,1+p_{B} p_{C}+q_{B} q_{C}=0 .
\end{gathered}
$$

Solving these equations with integer unknowns we see that there exist $\epsilon, \epsilon^{\prime} \in$ $\{1,-1\}$ so that (up to interchanging $x, y$ ) we have

$$
\left(p_{A}, q_{A}\right)=\left(2 \epsilon, \epsilon^{\prime}\right),\left(p_{B}, q_{B}\right)=\left(0,-\epsilon^{\prime}\right),\left(p_{C}, q_{C}\right)=\left(-\epsilon, \epsilon^{\prime}\right) .
$$

Then $\epsilon \gamma_{x}=2 \rho_{12}-\rho_{6}, \epsilon^{\prime} \gamma_{y}=\rho_{12}-\rho_{16}+\rho_{6}$. Hence

$2 \rho_{12}-\rho_{6}, \rho_{12}-\rho_{16}+\rho_{6}$ are \pm virtual cells.

The same argument shows that $2 \rho_{12}-\rho_{6^{\prime}}, \rho_{12}-\rho_{16}+\rho_{6^{\prime}}$ are \pm virtual cells. A similar (but simpler) argument shows that $\rho_{4}-\rho_{16}$ is \pm a virtual cell.

Assume now that we are in the setup of 22.27 (Case 1). By 22.27,

$$
\rho_{1}+\rho_{2}, \rho_{1}+\rho_{8}, \rho_{2}+\rho_{9}, \rho_{8}+\rho_{9}, \rho_{1}^{\dagger}+\rho_{2}^{\dagger}, \rho_{1}^{\dagger}+\rho_{8}^{\dagger}, \rho_{2}^{\dagger}+\rho_{9}^{\dagger}, \rho_{8}^{\dagger}+\rho_{9}^{\dagger},
$$

are constructible, hence by 22.2, 21.4 are of the form $n_{d} \gamma_{d}$ for suitable $d \in \mathcal{D}$, hence are \pm virtual cells. By an argument similar to that above (but simpler) we see that

$$
\rho_{1}-\rho_{2}, \rho_{1}-\rho_{8}, \rho_{2}-\rho_{9}, \rho_{8}-\rho_{9}, \rho_{1}^{\dagger}-\rho_{2}^{\dagger}, \rho_{1}^{\dagger}-\rho_{8}^{\dagger}, \rho_{2}^{\dagger}-\rho_{9}^{\dagger}, \rho_{8}^{\dagger}-\rho_{9}^{\dagger},
$$

are \pm virtual cells.

24.7. Assume that we are in the setup of 22.29. By 22.29, $\rho_{2}+\rho_{2}^{\prime}$ is constructible, hence by $22.2,21.4$, is of the form $n_{d} \gamma_{d}$ for some $d \in \mathcal{D}$, hence is \pm a virtual cell. As in 24.6, we see that $\rho_{2}-\rho_{2}^{\prime}$ is \pm a virtual cell.

\section{Relative Coxeter groups}

25.1. Let $W, S$ be a Coxeter group and let $u \in A_{W}$ (see 1.17). We assume that $W$ is a Weyl group or an affine Weyl group. Let $J$ be a $u$-stable subset of $S$ such that $W_{J}$ is finite (that is, $J \neq S$ when $W$ is infinite). Let $U: W \rightarrow\{$ permutations of $R\}$ be as in 1.5. Let $\mathcal{W}$ be the set of all $w \in W$ such that $U(w)$ carries $\{(1, s) ; s \in J\}$ onto itself. (A subgroup of $W$.) Alternatively,

$$
\mathcal{W}=\left\{w \in W ; w W_{J}=W_{J} w, w \text { has minimal length in } w W_{J}=W_{J} w\right\}
$$


Let $K$ be the set of all $u$-orbits $k$ on $S-J$ such that $W_{J \cup k}$ is finite. (In the case where $W$ is infinite, $K$ consists of all $u$-orbits on $S-J$ if $\sharp(u \backslash(S-J)) \geq 2$ and $K=\emptyset$ if $\sharp(u \backslash(S-J))=1$.) We assume that $J$ is $u$-excellent in the following sense: for any $k \in K$ we have $w_{0}^{J \cup k} J w_{0}^{J \cup k}=J$.

For $k \in K$ we have $w_{0}^{J \cup k} w_{0}^{J} w_{0}^{J \cup k}=w_{0}^{J}$ hence

$$
\tau_{k}:=w_{0}^{J \cup k} w_{0}^{J}=w_{0}^{J} w_{0}^{J \cup k}
$$

satisfies $\tau_{k}^{2}=1$.

If $k \in K$ then $U\left(w_{0}^{J \cup k}\right)$ maps $\{(1, s) ; s \in J \cup k\}$ onto $\{(-1, s) ; s \in J \cup k\}$. It also maps $\{( \pm 1, s) ; s \in J\}$ onto $\{( \pm 1, s) ; s \in J\}$. Hence it maps $\{(1, s) ; s \in J\}$ onto $\{(-1, s) ; s \in J\}$. Similarly, $U\left(w_{0}^{J}\right)$ maps $\{(-1, s) ; s \in J\}$ onto $\{(1, s) ; s \in J\}$. Hence $U\left(\tau_{k}\right)=U\left(w_{0}^{J}\right) U\left(w_{0}^{J \cup k}\right)$ maps $\{(1, s) ; s \in J\}$ onto $\{(1, s) ; s \in J\}$. Thus, $\tau_{k} \in \mathcal{W}$. More precisely, $\tau_{k} \in \mathcal{W}^{u}$, the fixed point set of $u: \mathcal{W} \rightarrow \mathcal{W}$.

The following result is proved in [L1] assuming that $W$ is a Weyl group (see [L11] for the case where $W$ is an affine Weyl group).

(a) $\mathcal{W}^{u}$ is a Coxeter group on the generators $\left\{\tau_{k} ; k \in K\right\}$. Moreover, if $W$ is a Weyl group then $\mathcal{W}^{u}$ is a Weyl group; if $W$ is an affine Weyl group and $\sharp(u \backslash(S-J)) \geq 2$ then $\mathcal{W}^{u}$ is an affine Weyl group; if $W$ is an affine Weyl group and $\sharp(u \backslash(S-J))=1$ then $\mathcal{W}^{u}=\{1\}$.

25.2. We now strengthen our assumption on $J$ by assuming that there exists an adjoint reductive group $G_{J}$ defined over $\mathbf{F}_{q}$ whose Coxeter graph is $J$ (a full subgraph of the Coxeter graph of $W$ ), such that $u: J \rightarrow J$ is induced by the Frobenius map of $G_{J}$ and that $G_{J}\left(\mathbf{F}_{q}\right)$ admits a unipotent cuspidal representation $E$; let $\mathbf{c}_{0}$ be the two-sided cell of $W_{J}$ (with the weight function given by length) corresponding to this unipotent representation in the classification [L5]. The function $\mathbf{a}: W \rightarrow \mathbf{N}$ (see 13.6) (defined in terms of the weight function given by the length) takes a constant value $a$ on $\mathbf{c}_{0}$ and a constant value $a_{k}$ on $\mathbf{c}_{0} \tau_{k}$ for $k \in K$ (see 9.13, P.11, 15.6). The function $\left\{\tau_{k} ; k \in K\right\} \rightarrow \mathbf{Z}$ given by $\tau_{k} \mapsto a_{k}-a$ takes equal values at two elements $\tau_{k}, \tau_{k^{\prime}}$ that are conjugate in $\mathcal{W}^{u}$ (case by case check) hence it is the restriction of a weight function $L: \mathcal{W}^{u} \rightarrow \mathbf{Z}$. This weight function takes $>0$ values on $\left\{\tau_{k} ; k \in K\right\}$. Let $\mathbf{a}_{L}: \mathcal{W}^{u} \rightarrow \mathbf{N}$ be the function defined like $\mathbf{a}: W \rightarrow \mathbf{N}$ (see 13.6) in terms of $\mathcal{W}^{u}$ (instead of $W$ ) and the weight function just defined. Define $\mathbf{a}^{\prime}: \mathcal{W}^{u} \rightarrow \mathbf{N}$ by $\mathbf{a}^{\prime}(x)=\mathbf{a}(y x)$ where $y$ is any element of $\mathbf{c}_{0}$. This is independent of the choice of $y$, by 9.13 , P.11, 15.6 .

Conjecture 25.3. (a) $\mathbf{a}_{L}=\mathbf{a}^{\prime}$.

(b) Let $\mathbf{c}$ be a two-sided cell of $\mathcal{W}^{u}$ (relative to the weight function $L$ ) as in 25.2. There exists a (necessarily unique) two-sided cell $\tilde{\mathbf{c}}$ of $W$ (relative to the weight function given by length) such that $y x \in \tilde{\mathbf{c}}$ for any $y \in \mathbf{c}_{0}, x \in \mathbf{c}$. Moreover the $\operatorname{map} \mathbf{c} \mapsto \tilde{\mathbf{c}}$ is injective.

This would reduce the problem of computing the two-sided cells of $\mathcal{W}^{u}$ (relative to the weight function $L$ ) to the analogous problem for $W$ (relative to the weight function given by length). 


\section{REPRESENTATIONS}

26.1. Let $W, S$ be an affine Weyl group and let $u \in A_{W}$ (see 1.17). Let $J$ be a $u$-stable subset of $S$ with $J \neq S$. Let $\mathcal{U}(J)$ be the set of isomorphism classes of unipotent cuspidal representations of $G_{J}\left(\mathbf{F}_{q}\right)$ (as in 25.2). Note that $\mathcal{U}(J)$ is independent of the choice of $G_{J}$. Let $E \in \mathcal{U}(J)$. Let $\mathcal{H}(W, J, E)$ be the IwahoriHecke algebra attached to $\mathcal{W}^{u}$ (defined as in 25.1 in terms of $W, S, J$ ) and to the weight function $L: \mathcal{W}^{u} \rightarrow \mathbf{N}$ (defined as in 25.2). Let $\Omega$ be as in 1.18. Let

$$
\Omega^{u}=\{a \in \Omega ; u a=a u\}, \Omega^{u, J}=\left\{a \in \Omega^{u} ; a(J)=J\right\} .
$$

If $a \in \Omega^{u, J}$ then $a: W \rightarrow W$ restricts to an automorphism of $\mathcal{W}^{u}$ as a Coxeter group; this automorphism is compatible with the weight function $L: \mathcal{W}^{u} \rightarrow \mathbf{N}$ hence it induces an automorphism of the algebra $\mathcal{H}(W, J, E)$. Hence we may form a semidirect product algebra $\mathcal{H}(W, J, E) \otimes_{\mathcal{A}} \mathcal{A}\left[\Omega^{u, J}\right]$ where $\mathcal{A}\left[\Omega^{u, J}\right]$ is the group algebra of $\Omega^{u, J}$ over $\mathcal{A}$.

Let $v_{0} \in \mathbf{C}^{*}$ be such that $v_{0}=1$ or $v_{0}$ is not a root of 1 . Let

$$
\left(\mathcal{H}(W, J, E) \otimes_{\mathcal{A}} \mathcal{A}\left[\Omega^{u, J}\right]\right)_{v_{0}}
$$

be the $\mathbf{C}$-algebra obtained from $\mathcal{H}(W, J, E) \otimes_{\mathcal{A}} \mathcal{A}\left[\Omega^{u, J}\right]$ by the change of scalars $\mathcal{A} \rightarrow \mathbf{C}, v \mapsto v_{0}$. Let

$$
\mathcal{I}=\sqcup \operatorname{Irr}\left(\mathcal{H}(W, J, E) \otimes_{\mathcal{A}} \mathcal{A}\left[\Omega^{u, J}\right]\right)_{v_{0}}
$$

where Irr stands for the set of isomorphism classes of simple modules of an algebra and the disjoint union is taken over all $(J, E)$ as above modulo the action of $\Omega^{u}$.

On the other hand, let $\mathcal{G}$ be a connected, simply connected almost simple reductive group over $\mathbf{C}$, of type "dual" to that of $W$. Let $A(\mathcal{G})$ be the group of automorphisms of $\mathcal{G}$ modulo the group of inner automorphisms of $\mathcal{G}$. There is a natural action of $A(\mathcal{G})$ on $\mathcal{G}$ (well defined up to conjugacy) and we form the semidirect product $\tilde{\mathcal{G}}$ of $\mathcal{G}$ and $A(\mathcal{G})$ via this action. Note that $\mathcal{G}$ may be identified with the identity component of $\tilde{\mathcal{G}}$. Let $\mathcal{J}$ be the set of all pairs $(C, \mathcal{E})$ where $C$ is a $\mathcal{G}$-conjugacy class in $\tilde{\mathcal{G}}$ and $\mathcal{E}$ is an irreducible $\mathcal{G}$-equivariant local system on $C$.

Theorem 26.2. There is a natural bijection $\mathcal{I} \leftrightarrow \mathcal{J}$.

This is shown in [L11],[L13]. Using this bijection we may transfer the partition of $\mathcal{I}$ into pieces indexed by the various $(J, E)$ into a partition of $\mathcal{J}$ into pieces again indexed by the various $(J, E)$. This partition can be described purely in terms of the geometry of $\tilde{\mathcal{G}}$ (see [L13]).

\section{A neW ReAlization of HeCke Algebras}

27.1. Let $G, F, \mathcal{P}, \mathbf{E}, W, S, J, \mathcal{W}, u, \ldots$ be as in 0.3 . Let $H=H\left(G^{F}, \mathcal{P}^{F}, \mathbf{E}\right)$. In this section we give a new realization of the Hecke algebra $H$ as a function space. 
We will identify $\overline{\mathbf{Q}}_{l}=\mathbf{C}$ (where $l$ is a prime number invertible in $\mathbf{F}_{q}$ ) via some field isomorphism.

Let $P_{0} \in \mathcal{P}^{F}$. Let $L$ be an $F$-stable Levi subgroup of $P_{0}, N L$ the normalizer of $L$ in $G, Z_{L}$ the centre of $L$. Let $M=N L / Z_{L}$. We have canonically $N L / L=\mathcal{W}$ hence $M=\sqcup_{w \in \mathcal{W}} M_{w}$ where $M_{w}$ is the inverse image of $w$ under the obvious map $N L / Z_{L} \rightarrow N L / L$. We have $M_{1}=L / Z_{L}=L_{a d}$. The conjugation action defines an (injective) homomorphism $M \rightarrow A u t(L)$ which restricts, for any $w \in \mathcal{W}$, to an

(a) isomorphism of $M_{w}$ onto an $L_{a d}$-coset $\operatorname{Aut}(L)_{w}$ in $\operatorname{Aut}(L)$.

By known properties of unipotent representations, there is a unique $L_{a d}^{F}$-module structure on $\mathbf{E}_{P_{0}}$ that extends the given $P_{0}^{F}$-module structure on $\mathbf{E}_{P_{0}}$ via the obvious homomorphism $P_{0}^{F} \rightarrow L_{a d}^{F}$. We choose an $M^{F}$-module structure $\iota$ : $M^{F} \rightarrow G L\left(\mathbf{E}_{P_{0}}\right)$ on $\mathbf{E}_{P_{0}}$ that extends this $L_{a d}^{F}$-module structure. (This exists by known properties of unipotent cuspidal representations.)

Let $w \in \mathcal{W}$ and let $\mathcal{O}_{w}$ be the corresponding good $G$-orbit on $\mathcal{P} \times \mathcal{P}$. For $\left(P_{1}, P_{2}\right) \in \mathcal{O}_{w}$ let $\bar{P}_{2} \stackrel{\psi_{P_{1}}^{P_{2}}}{\longrightarrow} \bar{P}_{1}$ be the unique isomorphism which takes the image of any $x \in P_{1} \cap P_{2}$ under $P_{1} \cap P_{2} \rightarrow \bar{P}_{2}$ to the image of $x \in P_{1} \cap P_{2}$ under $P_{1} \cap P_{2} \rightarrow \bar{P}_{1}$. Then the composition

$$
\bar{P}_{0} \stackrel{\operatorname{Ad}\left(g_{2}\right)}{\longrightarrow} \bar{P}_{2} \stackrel{\psi_{P_{1}}^{P_{2}}}{\longrightarrow} \bar{P}_{1} \stackrel{\operatorname{Ad}\left(g_{1}^{-1}\right)}{\longrightarrow} \bar{P}_{0}
$$

where $g_{1}, g_{2} \in G, g_{1} P_{0} g_{1}^{-1}=P_{1}, g_{2} P_{0} g_{2}^{-1}=P_{2}$, may be regarded as an element of $\operatorname{Aut}(L)_{w}$ (we identify $\bar{P}_{0}=L$ ). This corresponds under (a) to an element $\alpha_{g_{1}, g_{2}} \in M_{w}$.

27.2. Assume now that $F(w)=w$. Define ${ }^{w} \phi \in H$ as follows: if $\left(P_{1}, P_{2}\right) \in \mathcal{O}_{w}$ then $\left({ }^{w} \phi\right)_{P_{1}}^{P_{2}}: \mathbf{E}_{P_{2}} \rightarrow \mathbf{E}_{P_{1}}$ is the composition

$$
\mathbf{E}_{P_{2}} \stackrel{g_{2}^{-1}}{\longrightarrow} \mathbf{E}_{P_{0}} \stackrel{\iota\left(\alpha_{g_{1}, g_{2}}\right)}{\longrightarrow} \mathbf{E}_{P_{0}} \stackrel{g_{1}}{\longrightarrow} \mathbf{E}_{P_{1}}
$$

where $g_{1}, g_{2} \in G^{F}, g_{2} P_{0} g_{2}^{-1}=P_{2}, g_{1} P_{0} g_{1}^{-1}=P_{1}$; if $\left(P_{1}, P_{2}\right) \notin \mathcal{O}_{w}$ then $\left({ }^{w} \phi\right)_{P_{1}}^{P_{2}}$ : $\mathbf{E}_{P_{2}} \rightarrow \mathbf{E}_{P_{1}}$ is $0 .\left(\left({ }^{w} \phi\right)_{P_{1}}^{P_{2}}\right.$ is independent of the choices of $\left.g_{1}, g_{2}.\right)$

27.3. For $w$ as in 27.2 we have $\mathcal{O}_{w^{-1}}=\left\{\left(P_{2}, P_{1}\right) \in \mathcal{P} \times \mathcal{P} ;\left(P_{1}, P_{2}\right) \in \mathcal{O}_{w}\right\}$. Let $\mathcal{U}=\left\{P_{1} \in \mathcal{P}^{F} ;\left(P_{0}, P_{1}\right) \in \mathcal{O}_{w}\right\}$.

Then $\sharp \mathcal{U}=q^{l(w)}$ where $l$ is length in $W$. The composition $\left({ }^{w} \phi\right)\left({ }^{w^{-1}} \phi\right)$ has as $\left(P_{0}, P_{0}\right)$-component the sum over all $P_{1} \in \mathcal{U}$ of the compositions

$$
\mathbf{E}_{P_{0}} \stackrel{\iota\left(\alpha_{g_{1}, 1}\right)}{\longrightarrow} \mathbf{E}_{P_{0}} \stackrel{g_{1}}{\longrightarrow} \mathbf{E}_{P_{1}} \stackrel{g_{1}^{-1}}{\longrightarrow} \mathbf{E}_{P_{0}} \stackrel{\iota\left(\alpha_{1, g_{1}}\right)}{\longrightarrow} \mathbf{E}_{P_{0}}
$$

where $g_{1} \in G^{F}, g_{1} P_{0} g_{1}^{-1}=P_{1}$, that is $q^{l(w)}$ times the identity map of $\mathbf{E}_{P_{0}}$. Thus,

(a) $\left({ }^{w} \phi\right)\left({ }^{w^{-1}} \phi\right)=q^{l(w)}\left({ }^{1} \phi\right)+$ linear combination of $w^{\prime} \phi$ with $w^{\prime} \neq 1$. 
27.4. Let $w, w^{\prime} \in \mathcal{W}^{u}$ be such that $l\left(w w^{\prime}\right)=l(w)+l\left(w^{\prime}\right)$. (Here $l$ is length in $W$.) Then

(a) $\left(P_{1}, P_{2}\right) \in \mathcal{O}_{w},\left(P_{2}, P_{3}\right) \in \mathcal{O}_{w^{\prime}} \Longrightarrow\left(P_{1}, P_{3}\right) \in \mathcal{O}_{w w^{\prime}}$,

(b) if $\left(P_{1}, P_{3}\right) \in \mathcal{O}_{w w}$ then there is a unique $P_{2} \in \mathcal{P}$ such that $\left(P_{1}, P_{2}\right) \in$ $\mathcal{O}_{w},\left(P_{2}, P_{3}\right) \in \mathcal{O}_{w^{\prime}}$.

If $P_{1}, P_{2}, P_{3}$ are as in (a) we have $\psi_{P_{1}}^{P_{3}}=\psi_{P_{1}}^{P_{2}} \psi_{P_{2}}^{P_{3}}: \mathbf{E}_{P_{3}} \rightarrow \mathbf{E}_{P_{1}}$. From the definitions we see that

(c) $\left({ }^{w} \phi\right)\left(w^{\prime} \phi\right)={ }^{w} w^{\prime} \phi$.

27.5. For $w \in \mathcal{W}^{u},{ }^{w} \phi$ is a basis element of $H_{\mathcal{O}_{w}}$. If $w=t_{k_{1}} t_{k_{2}} \ldots t_{k_{r}}$ is a reduced expression in $\mathcal{W}^{u}$ (see 0.3) then $l(w)=l\left(t_{k_{1}}\right)+l\left(t_{k_{2}}\right)+\cdots+l\left(t_{k_{r}}\right)$ (where $l$ is as in 27.4) and $T_{w}=T_{\tau_{k_{1}}} T_{\tau_{k_{2}}} \ldots T_{\tau_{k_{r}}}$ (notation of 0.3 ) is a well defined basis element of $H_{\mathcal{O}_{w}}$ independent of the reduced expression. Hence ${ }^{w} \phi=x_{w} T_{w}$ where

(a) $x_{w}=x_{\tau_{k_{1}}} x_{\tau_{k_{2}}} \ldots x_{\tau_{k_{r}}}$

with $x_{w} \in \mathbf{C}^{*}$ for all $w \in \mathcal{W}^{u}$. From $27.4(\mathrm{c})$ we see that ${ }^{1} \phi$ is the unit element of $H$. Hence ${ }^{1} \phi=T_{1}$. By $27.3(\mathrm{a})$, we have $\left({ }^{\tau_{k}} \phi\right)\left({ }^{\tau_{k}} \phi\right)=q^{l\left(\tau_{k}\right)}\left({ }^{1} \phi\right)+\ldots$ hence

(b) $x_{\tau_{k}}^{2} T_{\tau_{k}}^{2}=q^{l\left(\tau_{k}\right)} T_{1}+$ linear combination of $T_{w^{\prime}}$ with $w^{\prime} \neq 1$.

On the other hand, by $0.3(\mathrm{~d})$ we have $T_{\tau_{k}}^{2}=\left(q^{N_{k} / 2}-q^{-N_{k} / 2}\right) T_{\tau_{k}}+T_{1}$. Comparing with (b) we see that $x_{\tau_{k}}^{2}=q^{l\left(\tau_{k}\right)}$ hence $x_{\tau_{k}}=\epsilon_{k} q^{l\left(\tau_{k}\right) / 2}$ where $\epsilon_{k} \in\{1,-1\}$. From (a) we see that for $w \in \mathcal{W}^{u}$ we have $x_{w}=\epsilon_{w} q^{l(w) / 2}$ where $w \mapsto \epsilon_{w}$ is a function $\mathcal{W}^{u} \rightarrow\{1,-1\}$ satisfying $\epsilon_{k} \epsilon_{k^{\prime}} \epsilon_{k} \cdots=\epsilon_{k^{\prime}} \epsilon_{k} \epsilon_{k^{\prime}} \ldots$ for $k \neq k^{\prime}$ (both products have a number of terms equal to the order of $\tau_{k} \tau_{k^{\prime}}$ in $\left.\mathcal{W}^{u}\right)$. It follows that $w \mapsto \epsilon_{w}$ is a group homomorphism $\mathcal{W}^{u} \rightarrow\{1,-1\}$. Since $M^{F} / L_{a d}^{F}=\mathcal{W}^{u}$, we may regard $\epsilon$ as a homomorphism $M^{F} \rightarrow \mathbf{C}^{*}$ which is trivial on $L_{a d}^{F}$.

27.6. Replacing $\iota: M^{F} \rightarrow G L\left(\mathbf{E}_{P_{0}}\right)$ by its tensor product with $\epsilon: M^{F} \rightarrow \mathbf{C}^{*}$ we obtain a new homomorphism $\iota_{0}: M^{F} \rightarrow G L\left(\mathbf{E}_{P_{0}}\right)$. If we now redefine ${ }^{w} \phi$ in terms of $\iota_{0}$ rather than $\iota$, then the $\epsilon$-factors disappear and we have

(a) ${ }^{w} \phi=q^{l(w) / 2} T_{w}, w \in \mathcal{W}^{u}$.

Note that $\iota_{0}$ is uniquely determined by property (a) and by its restriction to $L_{a d}^{F}$.

27.7. Let $D=\operatorname{dim} \mathbf{E}_{P_{0}}$. Let $Y$ be the set of all triples $\left(P, P^{\prime}, g U_{P}\right)$ where $P, P^{\prime} \in$ $\mathcal{P}$ and $g U_{P} \in G / U_{P}$ is such that $g P g^{-1}=P^{\prime}$ (hence $g U_{P}=U_{P^{\prime}} g$ ). Now $Y$ is naturally defined over $\mathbf{F}_{q}$, with Frobenius map

$F:\left(P, P^{\prime}, g U_{P}\right) \rightarrow\left(F(P), F\left(P^{\prime}\right), F(g) U_{F(P)}\right)$.

Let $Y_{0}$ be the set of all triples $\left(P, P^{\prime}, g U_{P}^{F}\right)$ where $P, P^{\prime} \in \mathcal{P}^{F}$ and $g U_{P}^{F} \in G^{F} / U_{P}^{F}$ is such that $g P g^{-1}=P^{\prime}$ (hence $g U_{P}^{F}=U_{P^{\prime}}^{F} g$ ). We have a bijection $Y_{0} \stackrel{\sim}{\longrightarrow} Y^{F}$ given by $\left(P, P^{\prime}, g U_{P}^{F}\right) \mapsto\left(P, P^{\prime}, g U_{P}\right)$.

Let $\mathfrak{B}$ be the vector space of all functions $f: Y_{0} \rightarrow \mathbf{C}$. We define a multiplication $\mathfrak{B} \times \mathfrak{B} \rightarrow \mathfrak{B}, f^{\prime}, f^{\prime \prime} \mapsto f^{\prime} * f^{\prime \prime}$ by

$$
\left(f^{\prime} * f^{\prime \prime}\right)\left(P, P^{\prime}, g U_{P}^{F}\right)=\frac{D}{\sharp \bar{P}^{F}} \sum_{\tilde{P}, g^{\prime} U_{P}^{F}, g^{\prime \prime} U_{\tilde{P}}^{F}} f^{\prime}\left(P, \tilde{P}, g^{\prime} U_{P}^{F}\right) f^{\prime \prime}\left(\tilde{P}, P^{\prime}, g^{\prime \prime} U_{\tilde{P}}^{F}\right)
$$


where the sum is taken over all

$$
\tilde{P} \in \mathcal{P}^{F}, g^{\prime} U_{P}^{F} \in G^{F} / U_{P}^{F}, g^{\prime \prime} U_{\tilde{P}}^{F} \in G^{F} / U_{\tilde{P}}^{F}
$$

such that

$$
g^{\prime} P g^{\prime-1}=\tilde{P}, g^{\prime \prime} \tilde{P} g^{\prime \prime-1}=P^{\prime}, g^{\prime \prime} g^{\prime} \in U_{P^{\prime}}^{F} g=g U_{P}^{F}
$$

Equivalently,

$$
\left(f^{\prime} * f^{\prime \prime}\right)\left(P, P^{\prime}, g U_{P}^{F}\right)=\frac{D}{\sharp \bar{P}^{F}} \sharp\left(U_{P}^{F}\right)^{-1} \sum_{\tilde{P}, g^{\prime}} f^{\prime}\left(P, \tilde{P}, g^{\prime} U_{P}^{F}\right) f^{\prime \prime}\left(\tilde{P}, P^{\prime}, g g^{\prime-1} U_{\tilde{P}}^{F}\right)
$$

where the sum is taken over all $\tilde{P} \in \mathcal{P}^{F}, g^{\prime} \in G^{F}$ such that $g^{\prime} P g^{\prime-1}=\tilde{P}$. With this multiplication, $\mathfrak{B}$ becomes an associative algebra.

Define $\kappa: H \rightarrow \mathfrak{B}$ by $\phi \mapsto \kappa(\phi)$ where $\kappa(\phi)\left(P, P^{\prime}, g U_{P}^{F}\right)$ is the trace of the composition

$$
\mathbf{E}_{P} \stackrel{g}{\rightarrow} \mathbf{E}_{P^{\prime}} \stackrel{\phi_{P}^{P^{\prime}}}{\longrightarrow} \mathbf{E}_{P}
$$

(This is independent of the choice of $g$ in its $U_{P}^{F}$-coset; $\phi_{P}^{P^{\prime}}$ is as in 0.1.)

Lemma 27.8. $\kappa: H \rightarrow \mathfrak{B}$ is an algebra homomorphism.

Let $\phi, \phi^{\prime} \in H$ and let $\left(P, P^{\prime}, g U_{P}^{F}\right) \in Y_{0}$. Then

$$
\begin{aligned}
& \kappa\left(\phi \phi^{\prime}\right)\left(P, P^{\prime}, g U_{P}^{F}\right)=\operatorname{tr}\left(\mathbf{E}_{P} \stackrel{g}{\rightarrow} \mathbf{E}_{P^{\prime}} \stackrel{\left(\phi \phi^{\prime}\right)_{P}^{P^{\prime}}}{\longrightarrow} \mathbf{E}_{P}\right) \\
& =\sum_{\tilde{P}} \operatorname{tr}\left(\mathbf{E}_{P} \stackrel{g}{\rightarrow} \mathbf{E}_{P^{\prime}} \stackrel{\phi_{\tilde{P}}^{\prime P^{\prime}}}{\longrightarrow} \mathbf{E}_{\tilde{P}} \stackrel{\phi_{P}^{\tilde{P}}}{\longrightarrow} \mathbf{E}_{P}\right) .
\end{aligned}
$$

On the other hand,

$$
\begin{aligned}
& \kappa(\phi) * \kappa\left(\phi^{\prime}\right)\left(P, P^{\prime}, g U_{P}^{F}\right) \\
& =\sharp\left(U_{P}^{F}\right)^{-1} \frac{D}{\sharp \bar{P}^{F}} \sum_{\tilde{P}, g^{\prime} ; g^{\prime} P g^{\prime-1}=\tilde{P}} \kappa(\phi)\left(P, \tilde{P}, g^{\prime} U_{P}^{F}\right) \kappa\left(\phi^{\prime}\right)\left(\tilde{P}, P^{\prime}, g g^{\prime-1} U_{\tilde{P}}^{F}\right) \\
& =\frac{D}{\sharp P^{F}} \sum_{\tilde{P}, g^{\prime} ; g^{\prime} P g^{\prime-1}=\tilde{P}} \operatorname{tr}\left(\mathbf{E}_{P} \stackrel{g^{\prime}}{\longrightarrow} \mathbf{E}_{\tilde{P}} \stackrel{\phi_{P}^{\tilde{P}}}{\longrightarrow} \mathbf{E}_{P}\right) \operatorname{tr}\left(\mathbf{E}_{\tilde{P}} \stackrel{g g^{\prime-1}}{\longrightarrow} \mathbf{E}_{P^{\prime}} \stackrel{\phi_{\tilde{P}}^{P^{\prime}}}{\longrightarrow} \mathbf{E}_{\tilde{P}}\right) \\
& =\frac{D}{\sharp P^{F}} \sum_{\tilde{P}, g^{\prime} ; g^{\prime} P g^{\prime-1}=\tilde{P}} \operatorname{tr}\left(\mathbf{E}_{P} \stackrel{g^{\prime}}{\longrightarrow} \mathbf{E}_{\tilde{P}} \stackrel{\phi_{P}^{\tilde{P}}}{\longrightarrow} \mathbf{E}_{P}\right) \operatorname{tr}\left(\mathbf{E}_{P} \stackrel{g}{\longrightarrow} \mathbf{E}_{P^{\prime}} \stackrel{\phi_{\tilde{P}}^{P^{\prime}}}{\longrightarrow} \mathbf{E}_{\tilde{P}} \stackrel{g^{\prime-1}}{\longrightarrow} \mathbf{E}_{P}\right) .
\end{aligned}
$$

It is then enough to show that for any $\tilde{P} \in \mathcal{P}^{F}$, we have

$$
\begin{aligned}
& \operatorname{tr}\left(\mathbf{E}_{P} \stackrel{g}{\rightarrow} \mathbf{E}_{P^{\prime}} \stackrel{\phi_{\tilde{P}}^{\prime P^{\prime}}}{\longrightarrow} \mathbf{E}_{\tilde{P}} \stackrel{\phi_{P}^{\tilde{P}}}{\longrightarrow} \mathbf{E}_{P}\right) \\
& =\frac{D}{\sharp P^{F}} \sum_{\substack{g^{\prime} \in G^{F} \\
g^{\prime} \stackrel{P}{P} g^{\prime-1}=\tilde{P}}} \operatorname{tr}\left(\mathbf{E}_{P} \stackrel{g^{\prime}}{\rightarrow} \mathbf{E}_{\tilde{P}} \stackrel{\phi_{P}^{\tilde{P}}}{\longrightarrow} \mathbf{E}_{P}\right) \operatorname{tr}\left(\mathbf{E}_{P} \stackrel{g}{\rightarrow} \mathbf{E}_{P^{\prime}} \stackrel{\phi_{\tilde{P}}^{P^{\prime}}}{\longrightarrow} \mathbf{E}_{\tilde{P}} \stackrel{g^{\prime-1}}{\longrightarrow} \mathbf{E}_{P}\right) .
\end{aligned}
$$


Let $\gamma \in G^{F}$ be such that $\gamma P \gamma^{-1}=\tilde{P}$. We rewrite the equality to be proved using the substitution $g^{\prime}=\gamma h$ :

$$
\operatorname{tr}\left(\mathbf{E}_{P} \stackrel{A B}{\longrightarrow} \mathbf{E}_{P}\right)=\frac{D}{\sharp\left(P^{F}\right)} \sum_{h \in P^{F}} \operatorname{tr}\left(\mathbf{E}_{P} \stackrel{A h}{\longrightarrow} \mathbf{E}_{P}\right) \operatorname{tr}\left(\mathbf{E}_{P} \stackrel{h^{-1} B}{\longrightarrow} \mathbf{E}_{P}\right)
$$

where $A$ is the composition $\mathbf{E}_{P} \stackrel{\gamma}{\rightarrow} \mathbf{E}_{\tilde{P}} \stackrel{\phi_{P}^{\tilde{P}}}{\longrightarrow} \mathbf{E}_{P}$ and $B$ is the composition $\mathbf{E}_{P} \stackrel{g}{\rightarrow}$ $\mathbf{E}_{P^{\prime}} \stackrel{\phi_{\tilde{P}}^{P^{\prime}}}{\longrightarrow} \mathbf{E}_{\tilde{P}} \stackrel{\gamma^{-1}}{\longrightarrow} \mathbf{E}_{P}$. (Then $A B$ is the composition $\mathbf{E}_{P} \stackrel{g}{\longrightarrow} \mathbf{E}_{P^{\prime}} \stackrel{\phi_{\tilde{P}}^{P^{\prime}}}{\longrightarrow} \mathbf{E}_{\tilde{P}} \stackrel{\phi_{P}^{\tilde{P}}}{\longrightarrow}$ $\mathbf{E}_{P}$.) Now (a) follows immediately from the Schur orthogonality relations for the matrix coefficients of the irreducible representation of $P^{F}$ on $\mathbf{E}_{P}$. The lemma is proved.

27.9. Let $w \in \mathcal{W}^{u}$. Let $f_{w}: Y_{0} \rightarrow \mathbf{C}$ be the image of $q^{-l(w) / 2}\left({ }^{w} \phi\right)$ (defined as in 27.2 in terms of $\left.\iota_{0}\right)$ under $\kappa: H \rightarrow \mathfrak{B}$.

If $\left(P_{1}, P_{2}, g U_{P_{1}}^{F}\right) \in Y_{0},\left(P_{1}, P_{2}\right) \notin \mathcal{O}_{w}$ then $f_{w}\left(P_{1}, P_{2}, g U_{P_{1}}^{F}\right)=0$.

If $\left(P_{1}, P_{2}, g U_{P_{1}}^{F}\right) \in Y_{0},\left(P_{1}, P_{2}\right) \in \mathcal{O}_{w}$ then $f_{w}\left(P_{1}, P_{2}, g U_{P_{1}}^{F}\right)$ is $q^{-l(w) / 2}$ times the trace of the composition

$$
\mathbf{E}_{P_{1}} \stackrel{g_{2}^{-1} g}{\longrightarrow} \mathbf{E}_{P_{0}} \stackrel{\iota_{0}\left(\alpha_{g_{1}, g_{2}}\right)}{\longrightarrow} \mathbf{E}_{P_{0}} \stackrel{g_{1}}{\longrightarrow} \mathbf{E}_{P_{1}}
$$

where $g_{1}, g_{2} \in G^{F}, g_{2} P_{0} g_{2}^{-1}=P_{2}, g_{1} P_{0} g_{1}^{-1}=P_{1}$; here we may assume that $g_{1}=g^{-1} g_{2}$ hence

$$
f_{w}\left(P_{1}, P_{2}, g U_{P_{1}}^{F}\right)=q^{-l(w) / 2} \operatorname{tr}\left(\mathbf{E}_{P_{0}} \stackrel{\iota_{0}\left(\alpha_{g^{-1} g_{2}, g_{2}}\right)}{\longrightarrow} \mathbf{E}_{P_{0}}\right)
$$

where $g_{2} \in G^{F}, g_{2} P_{0} g_{2}^{-1}=P_{2}$ (with $\alpha_{g^{-1} g_{2}, g_{2}}$ as in 27.1).

In particular, if $\left(P_{1}, P_{2}, g U_{P_{1}}^{F}\right) \in Y_{0}, P_{1} \neq P_{2}$ then $f_{1}\left(P_{1}, P_{2}, g U_{P_{1}}^{F}\right)=0$; if $P_{1} \in \mathcal{P}^{F}, g \in P_{1}^{F}$ then

$$
f_{1}\left(P_{1}, P_{1}, g U_{P_{1}}^{F}\right)=\operatorname{tr}\left(\mathbf{E}_{P_{1}} \stackrel{g}{\rightarrow} \mathbf{E}_{P_{1}}\right) .
$$

Thus, $f_{1}$ is not identically zero.

Here are some properties of the functions $f_{w}$ which follow immediately from the corresponding properties of the functions ${ }^{w} \phi$ using 27.8.

(a) $\left(f_{\tau_{k}}-q^{-N_{k} / 2} f_{1}\right)\left(f_{\tau_{k}}+q^{N_{k} / 2} f_{1}\right)=0$ for all $k$,

(b) $f_{w} f_{w^{\prime}}=f_{w w^{\prime}}, w, w^{\prime} \in \mathcal{W}^{u}, l\left(w w^{\prime}\right)=l(w)+l\left(w^{\prime}\right)$.

Let $\bar{H}=\kappa(H)$. This is a subalgebra of $\mathfrak{B}$ generated as a vector space by $\left\{f_{w} ; w \in\right.$ $\left.\mathcal{W}^{u}\right\}$. From (b) we see that $f_{1} f_{w}=f_{w} f_{1}=f_{w}$ for all $w \in \mathcal{W}^{u}$, hence $f_{1}$ is the unit element of the algebra $\bar{H}$. From (a) we see that $f_{\tau_{k}}$ is invertible in this algebra for any $k$ and then from (b) we see that $f_{w}$ is invertible in this algebra for any $w \in W$. Since $f_{1} \neq 0$ we have $f_{w} \neq 0$ for any $w \in \mathcal{W}^{u}$. Now the $f_{w}$ have disjoint supports. (The support of $f_{w}$ is contained in $Y_{w}^{F}$ where $Y_{w}=\left\{\left(P_{1}, P_{2}, g U_{P_{1}}^{F}\right) \in Y ;\left(P_{1}, P_{2}\right) \in\right.$ $\left.\mathcal{O}_{w}\right\}$.) It follows that the elements $f_{w}\left(w \in \mathcal{W}^{u}\right)$ are linearly independent in the vector space $\bar{H}$. Hence $\kappa: H \rightarrow \bar{H}$ is an isomorphism of algebras.

We have thus obtained a new model $\bar{H}$ for the Hecke algebra $H$ as the vector space of functions $f: Y_{0} \rightarrow \mathbf{C}$ spanned by the functions $f_{w}\left(w \in \mathcal{W}^{u}\right)$ with multiplication $*$ as in 27.7 . 
27.10. Let

$$
Z=\left\{\left(P^{\prime}, g U_{P_{0}}\right) ; P^{\prime} \in \mathcal{P}, g \in G / U_{P_{0}} ; g P_{0} g^{-1}=P^{\prime}\right\} .
$$

Let $w \in \mathcal{W}^{u}$. Let

$$
Z_{w}=\left\{\left(P^{\prime}, g U_{P_{0}}\right) \in Z,\left(P_{0}, P^{\prime}\right) \in \mathcal{O}_{w}\right\} .
$$

We have a morphism $\lambda: Z_{w} \rightarrow \operatorname{Aut}(L)_{w}=M_{w}$ where $\lambda\left(P^{\prime}, g U_{P_{0}}\right)$ is the composition

$$
L=\bar{P}_{0} \stackrel{\operatorname{Ad}(g)}{\longrightarrow} \bar{P}^{\prime} \stackrel{\psi_{P_{0}}^{P^{\prime}}}{\longrightarrow} \bar{P}_{0}=L
$$

Note that $\lambda$ is a smooth morphism with connected fibres.

Now $Z_{w}, M_{w}$ are naturally defined over $\mathbf{F}_{q}$ with Frobenius maps $F$ and $\lambda$ commutes with $F$. Hence $\lambda$ restricts to a map

$$
Z_{w}^{F} \stackrel{\lambda}{\rightarrow} M_{w}^{F}
$$

Define $f_{w}^{0}: Z_{w}^{F} \rightarrow \mathbf{C}$ by

$$
f_{w}^{0}\left(P^{\prime}, g U_{P_{0}}\right)=f_{w}\left(P_{0}, P^{\prime}, g U_{P_{0}}\right) .
$$

(Here $g \in G^{F} / U_{P_{0}}^{F}$.) We have

$$
f_{w}^{0}=q^{-l(w) / 2} \lambda^{*}\left(\chi\left(\iota_{0}\right)_{w}\right)
$$

where $\chi\left(\iota_{0}\right)_{w}: M_{w}^{F} \rightarrow \mathbf{C}$ is the character of $\iota_{0}: M^{F} \rightarrow G L\left(\mathbf{E}_{P_{0}}\right)$ restricted to $M_{w}^{F}$.

27.11. The obvious homomorphism $\operatorname{Aut}(L) \rightarrow \operatorname{Aut}\left(L_{a d}\right)$ defines for any $w \in \mathcal{W}^{u}$ an isomorphism of $M_{w}=\operatorname{Aut}(L)_{w}$ with a connected component of the reductive algebraic group $A u t\left(L_{a d}\right)$ with identity component $L_{a d}$. Hence we have the notion of character sheaf on $M_{w}$ (see [L9]). Let $\hat{M}_{w}$ be the set of isomorphism classes of character sheaves on $M_{w}$. Let $A \in \hat{M}_{w}$. Then $A$ is $L_{a d}$-equivariant for the conjugation action of $M_{w}$. Since $\lambda$ is smooth with connected fibres of fixed dimension, a suitable shift of $\lambda^{*}(A)$ is a simple perverse sheaf $\tilde{A}$ on $Z_{w}$. Let $\tilde{A}^{\sharp}$ be the unique simple perverse sheaf on $Z$, whose support is the closure in $Z$ of the support of $\tilde{A}$ and which satisfies $\left.\tilde{A}^{\sharp}\right|_{Z_{w}}=\tilde{A}$.

Let $\hat{M}_{w}^{F}$ be the set of all $A \in \hat{M}_{w}$ such that $F^{*} A \cong A$. For any $A \in \hat{M}_{w}^{F}$ we choose an isomorphism $\phi: F^{*} A \stackrel{\sim}{\longrightarrow} A$. There are induced isomorphisms $\phi: F^{*} \tilde{A} \stackrel{\sim}{\longrightarrow} \tilde{A}, \phi: F^{*} \tilde{A}^{\sharp} \stackrel{\sim}{\longrightarrow} \tilde{A}^{\sharp}$. Let

$$
\chi_{A, \phi}: M_{w}^{F} \rightarrow \overline{\mathbf{Q}}_{l}, \chi_{\tilde{A}, \phi}: Z_{w}^{F} \rightarrow \overline{\mathbf{Q}}_{l}, \chi_{\tilde{A}^{\sharp}, \phi}: Z^{F} \rightarrow \overline{\mathbf{Q}}_{l}
$$

be the corresponding characteristic functions (alternating sums of traces of Frobenius at stalks of cohomology sheaves at various $F$-fixed points). We have $c_{\tilde{A}, \phi}=$ $(-1)^{N} \lambda^{*}\left(c_{A, \phi}\right)$ where $N=\operatorname{dim} Z_{w}-\operatorname{dim} M_{w}$ and $c_{\tilde{A}, \phi}=\left.c_{\tilde{A}^{\sharp}, \phi}\right|_{Z_{w}^{F}}$. 
It is known [L9] that the functions $\chi_{A, \phi}$ (where $A$ runs through $\hat{M}_{w}^{F}$ ) form a basis for the vector space of functions $M_{w}^{F} \rightarrow \mathbf{C}$ that are constant on the orbits of $M^{0 F}$ (acting on $M_{w}^{F}$ by conjugation). Hence

$$
\chi\left(\iota_{0}\right)_{w}=\sum_{A \in \hat{M}_{w}^{F}} \xi_{A} \chi_{A, \phi}
$$

where $\xi_{A} \in \mathbf{C}$ are uniquely determined. Applying $\lambda^{*}$ to both sides we deduce

$$
q^{l(w) / 2} f_{w}^{0}=\sum_{A} \xi_{A}(-1)^{N} \chi_{\tilde{A}, \phi}
$$

Hence

$$
f_{w}^{0}=\left.q^{-l(w) / 2}(-1)^{N} \sum_{A} \xi_{A} \chi_{\tilde{A}^{\sharp}, \phi}\right|_{Z_{w}^{F}} \cdot
$$

The following conjecture provides a geometric interpretation of the polynomials $p_{y, w}$ (see 5.3) attached to the Coxeter group $\mathcal{W}^{u}$ with its weight function $L$ : $\mathcal{W}^{u} \rightarrow \mathbf{N}$.

Conjecture 27.12. Assume that $y \in \mathcal{W}^{u}$. We have

$$
\begin{gathered}
\left.q^{-l(w) / 2}(-1)^{N} \sum_{A} \xi_{A} \chi_{\tilde{A}^{\sharp}, \phi}\right|_{Z_{y}^{F}}=\left.p_{y, w}\right|_{v=\sqrt{q}} f_{y}^{0}, \\
\left.\sum_{A} \xi_{A} \chi_{\tilde{A}^{\sharp}, \phi}\right|_{Z^{F}-\cup_{y} \in \mathcal{W}^{u}} Z_{y}^{F}=0 .
\end{gathered}
$$

27.13. We now consider the special case where $\mathcal{P}$ is the set of Borel subgroups of $G$ and $\mathbf{E}$ is the trivial vector bundle $\mathbf{C}$. Then $\mathcal{W}=W$. In this case the homomorphism $\iota_{0}$ is trivial. For $w \in \mathcal{W}^{u}=W^{u}$ and $\left(P_{1}, P_{2}, g U_{P_{1}}^{F}\right) \in Y_{0}$ we have

$$
\begin{aligned}
& f_{w}\left(P_{1}, P_{2}, g U_{P_{1}}^{F}\right)=0 \text { if }\left(P_{1}, P_{2}\right) \notin \mathcal{O}_{w}, \\
& f_{w}\left(P_{1}, P_{2}, g U_{P_{1}}^{F}\right)=q^{-l(w) / 2}, \text { if }\left(P_{1}, P_{2}\right) \in \mathcal{O}_{w} .
\end{aligned}
$$

In particular, the functions in $\kappa(H)$ do not depend on the third coordinate $g U_{P_{1}}^{F}$ which can therefore be omitted. For $f^{\prime}, f^{\prime \prime}$ in $\kappa(H)$ we have

$$
\left(f^{\prime} * f^{\prime \prime}\right)\left(P, P^{\prime}\right)=\sum_{\tilde{P} \in \mathcal{P} F} f^{\prime}(P, \tilde{P}) f^{\prime \prime}\left(\tilde{P}, P^{\prime}\right)
$$

In the present case, Conjecture 27.12 states that $\left.p_{y, w}\right|_{v=\sqrt{q}}$ is (up to normalization) the restriction to $Z_{y}^{F}$ of the characteristic function of the intersection cohomology sheaf of the closure of $Z_{w}$ in $Z$. Equivalently, if for $w \in W^{u}$ we set

$\mathcal{P}_{w}=\left\{P^{\prime} \in \mathcal{P} ;\left(P_{0}, P^{\prime}\right) \in \mathcal{O}_{w}\right)$ then $\left.p_{y, w}\right|_{v=\sqrt{q}}$ is (up to normalization) the restriction to $\mathcal{P}_{y}^{F}$ of the characteristic function of the intersection cohomology sheaf of the closure of $\mathcal{P}_{w}$ in $\mathcal{P}$.

This property is known to be true; it is proved in [KL2] in the case where $u=1$ on $W$ and is stated in the general case in [L3]. 
27.14. Let $\mathbf{G}, F, \mathcal{P}, \mathbf{E}, W, S, J, \mathcal{W}, u, \ldots$ be as in 0.6 . Let $H=H\left(\mathbf{G}^{F}, \mathcal{P}^{F}, \mathbf{E}\right)$. Everything in 27.1-27.13 extends to this case (we replace $G$ by $\mathbf{G}$ throughout) with the following modifications. In the definition of $\mathfrak{B}$ (see 27.7) we must now restrict ourselves to functions $f: Y_{0} \rightarrow \mathbf{C}$ such that

$$
\left\{\left(P, P^{\prime}\right) \in \mathcal{P}^{F} \times \mathcal{P}^{F} ; f\left(P, P^{\prime}, g U_{P}^{F}\right) \neq 0 \text { for some } g \in \mathbf{G}^{F}\right\}
$$

is contained in the union of finitely many $\mathbf{G}$-orbits on $\mathcal{P} \times \mathcal{P}$. Also, when defining the multiplication $*$ in 27.7 only the definition 27.7 (a) makes now sense (in 27.7(b) the quantity $\sharp\left(U_{P}^{F}\right)$ is infinite hence does not make sense). In 27.13 one should use Iwahori subgroups instead of Borel subgroups.

\section{REFERENCES}

[Be] R.Bédard, Cells in two Coxeter groups, Commun. Alg. 14 (1986), 1253-1286.

[Bo] N.Bourbaki, Groupes et algèbres de Lie, Ch. 4,5,6, Hermann, Paris, 1968.

[B] K.Bremke, On generalized cells in affine Weyl groups, J. Algebra 191 (1997), 149-173.

[BM] K.Bremke and G.Malle, Reduced words and a length function for $G(e, 1, n)$, Indag. Math. 8 (1997), 453-469.

[DL] P.Deligne and G.Lusztig, Representations of reductive groups over finite fields, Ann.Math. 103 (1976), 103-161.

[G] M.Geck, Constructible characters, leading coefficients and left cells for finite Coxeter groups with unequal parameters, Represent.theory 6 (2002), 1-30.

[GP] M.Geck and G.Pfeiffer, Characters of finite Coxeter groups and Iwahori-Hecke algebras, London Math.Soc.Monographs 21, Clarendon Press, Oxford, 2000.

[H] P.N.Hoefsmit, Representations of Hecke algebras of finite groups with BN-pairs of classical type, Thesis, Univ. of British Columbia, Vancouver (1974).

[I] N.Iwahori, On the structure of the Hecke ring of a Chevalley group over a finite field, J.Fac.Sci.Tokyo Univ. 10 (1964), 215-236.

[IM] N.Iwahori and H.Matsumoto, On some Bruhat decomposition and the structure of the Hecke ring of p-adic Chevalley groups, Publ.Math. I.H.E.S. 25 (1965), 5-48.

[KL1] D.Kazhdan and G.Lusztig, Representations of Coxeter groups and Hecke algebras, Inv. Math. 53 (1979), 165-184.

[KL2] D.Kazhdan and G.Lusztig, Schubert varieties and Poincaré duality, Proc.Symp.Pure Math. 36 (1980), Amer. Math. Soc., 185-203.

[L1] G.Lusztig, Coxeter orbits and eigenspaces of Frobenius, Inv.Math. 28 (1976), 101-159.

[L2] _ Irreducible representations of finite classical groups, Inv.Math. 43 (1977), 125175.

[L3] Left cells in Weyl groups, Lie group representations, I, Lect. Notes Math. 1024, Springer, 1983, pp. 99-111.

[L4] Some examples of square integrable representations of semisimple p-adic groups, Trans.Amer.Math.Soc. 227 (1983), 623-653.

[L5] - Characters of reductive groups over a finite field, Ann.Math.Studies 10\%, Princeton Univ. Press, 1984, 384p.

[L6] Cells in affine Weyl groups, Algebraic groups and related topics, Adv.Stud.Pure Math. 6, North-Holland and Kinokuniya, Tokyo and Amsterdam, 1985, pp. 255-287.

[L7] Sur les cellules gauches des groupes de Weyl, C.R.Acad.Sci.Paris(A) 302 (1986), $5-8$.

[L8] _ Cells in affine Weyl groups, II, J.Algebra 109 (1987), 536-548. 
[L9] Introduction to character sheaves, Proc. Symp. Pure Math. 47(1) (1987), Amer. Math. Soc., 165-180.

[L10] Intersection cohomology methods in representation theory, Proc.Int.Congr.Math. Kyoto 1990, Springer, Tokyo, 1991, pp. 155-174.

[L11] Classification of unipotent representations of simple p-adic groups, Int. Math. Res. Notices 1995, 517-589.

[L12] _ Lectures on Hecke algebras with unequal parameters, MIT Lectures (1999), $\mathrm{RT} / 0108172$.

[L13] _ Classification of unipotent representations of simple p-adic groups, II, RT/0111248 (to appear).

[X] N.Xi, Representations of affine Hecke algebras, Lect. Notes Math., vol. 1587, Springer, 1994.

Department of Mathematics, M.I.T., Cambridge, MA 02139 